\title{
Development of a Generic Dual Fuel ECU for Common Rail Diesel Engine Control
}

\author{
A thesis \\ submitted in partial fulfilment \\ of the requirements for the Degree \\ of \\ Master of Engineering in Electronic \\ and Computer Systems Engineering \\ at
}

Victoria University of Wellington

by

Luke James Frogley

\section{VICTORIA UNIVERSITY OF WELLINGTON \\ Te Whare Wānanga o te Ūpoko o te Ika a Māui}

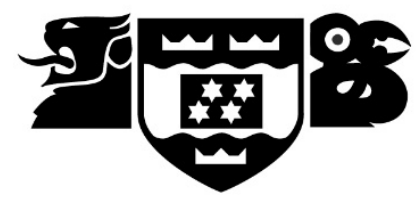

Victoria University of Wellington

2014 

For Mum and Dad 


\section{Abstract}

Rising costs of diesel fuel has led to an increased interest in dual fuel diesel engine conversion, which can offset diesel consumption though the simultaneous combustion of a secondary gaseous fuel. This system offers benefits both environmentally and financially in an increasingly energy-conscious society. Dual fuel engine conversions have previously been fitted to mechanical injection systems, requiring physical modification of the fuel pump. The aim of this work is to develop a novel electronic dual fuel control system that may be installed on any modern diesel engine using common rail fuel injection with solenoid injector valves, eliminating the need for mechanical modification of the diesel fuel system.

The dual fuel electronic control unit developed replaces up to 90 percent of the diesel fuel required with cleaner-burning and cheaper compressed natural gas, providing the same power output with lower greenhouse gas emissions than pure diesel. The dual fuel system developed controls the flow of diesel, gas, air, and engine timing to ensure combustion is optimised to maintain a specific torque at a given speed and demand. During controlled experimental analysis, the dual fuel system exceeded the target substitution rate of 90 precent, with a peak diesel substitution achieved of 97 percent, whilst maintaining the same torque performance of the engine under diesel operation. 


\section{Acknowledgements}

I would like to thank the following individuals for their help and support throughout the course of this project: firstly, my mother, father, and godfather who have been a continual source of motivational, emotional, and financial support, and for always believing in my abilities even when I did not. Next I would like to sincerely thank my supervisor Dale Carnegie, for giving me the technical and mental support I required, as well as the occasional stern email to keep me in line. I honestly could not have asked for a better supervisor or better person to work under. I would also like to thank Amber for putting up with me through the hard times and encouraging me in the better ones. I am immensely grateful for your encouraging words and countless cups of tea. To Harvey and Neil at DieselGas International: thank you for the opportunity for me to embark on this project, and for the huge wealth of knowledge I have gained through working alongside you both. I would also like to thank Danny from Cable Price Ltd., who provided me with an engine and equipment to test my project on, and Scott who continued to support my efforts with numerous out-calls to diagnose the many engine faults I managed to create. I must also thank the technical staff at Victoria University of Wellington who put up with my incessant borrowing of equipment (thanks Tim). Finally, to all of my family and friends who helped me get to the end of this project through your words of encouragement and support: thank you. 


\section{Contents}

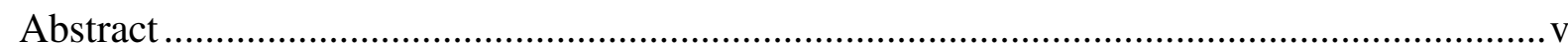

Acknowledgements .................................................................................................... vii

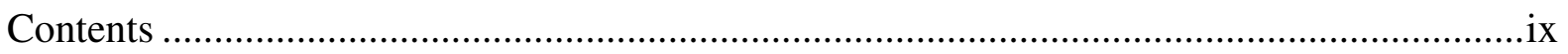

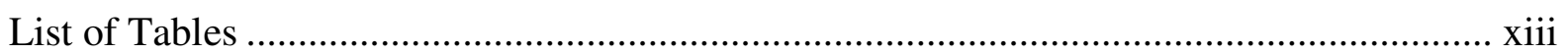

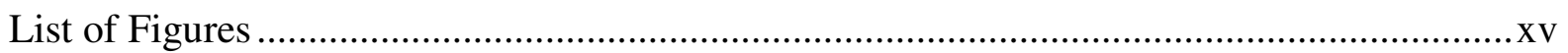

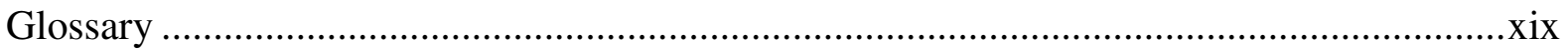

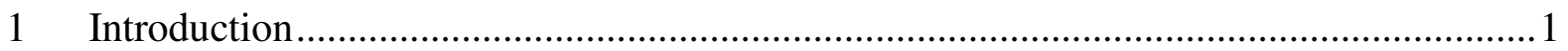

$1.1 \quad$ Overview/Motivation .................................................................................... 1

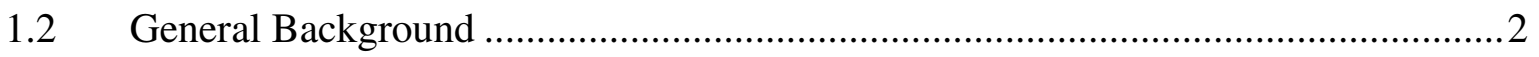

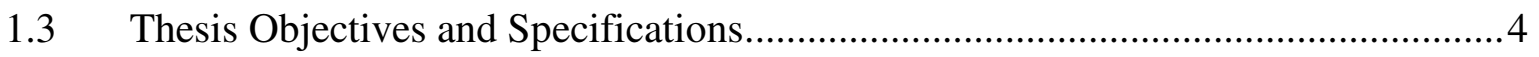

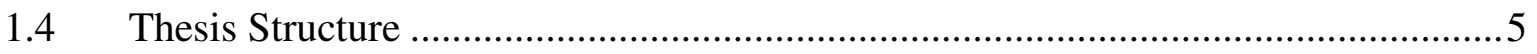

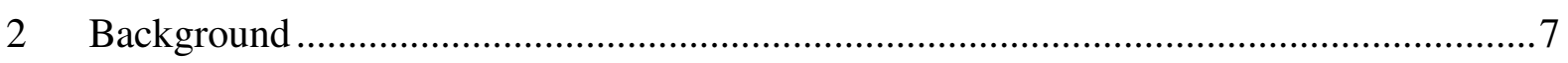

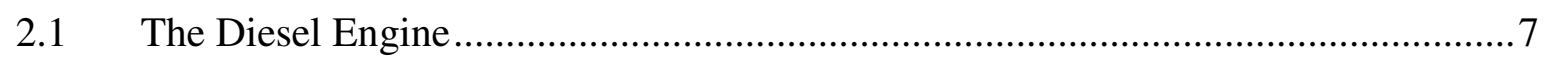

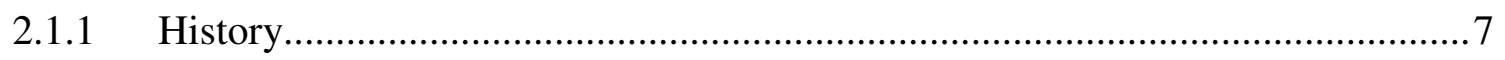

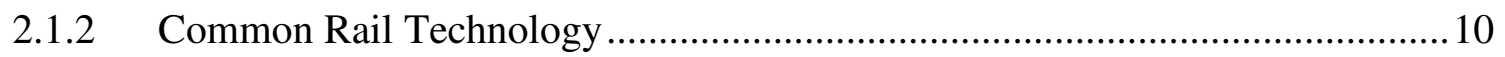

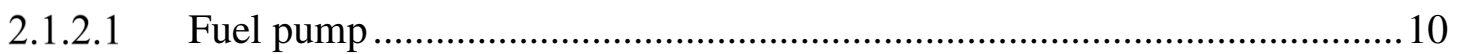

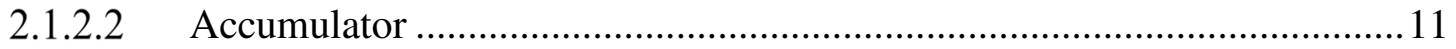

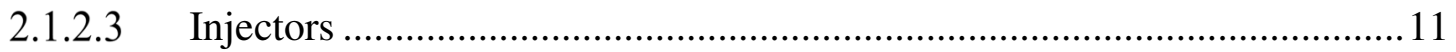

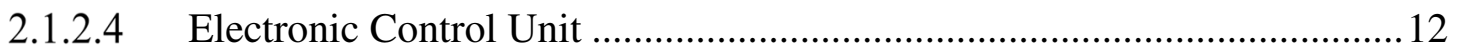

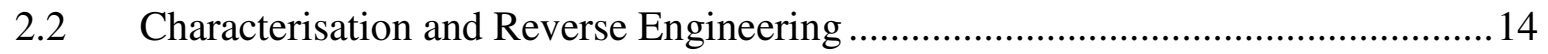

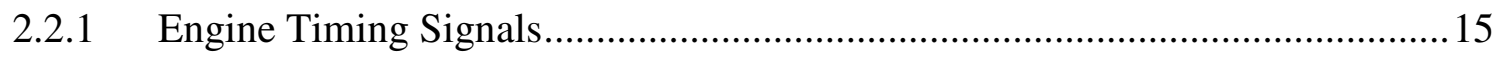

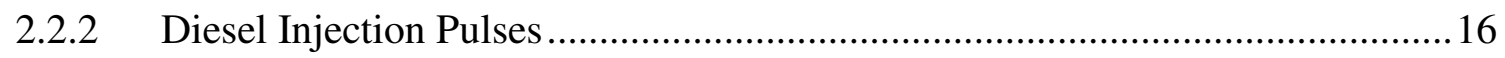

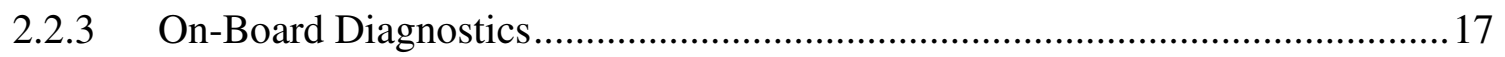

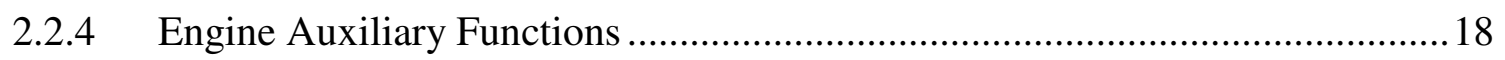




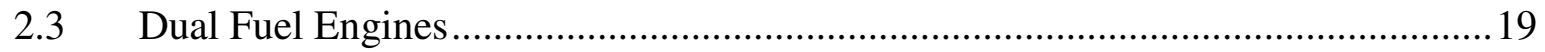

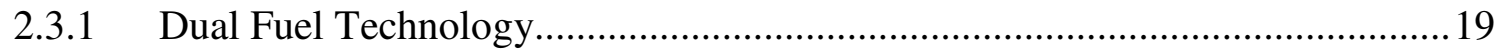

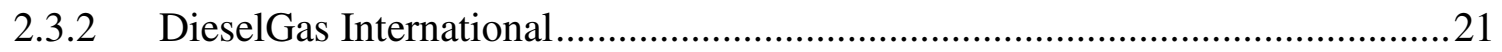

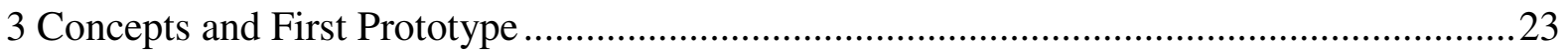

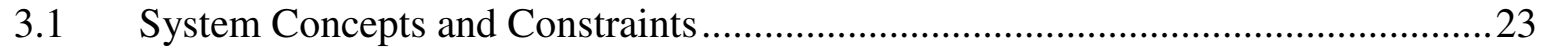

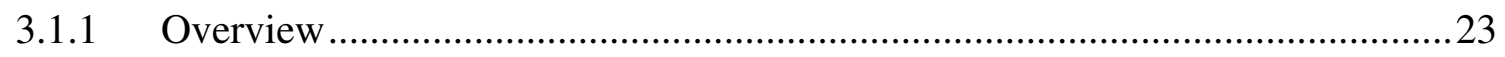

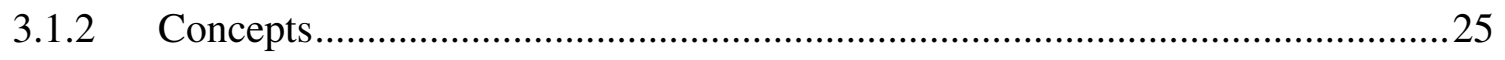

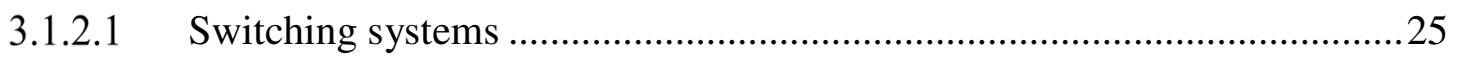

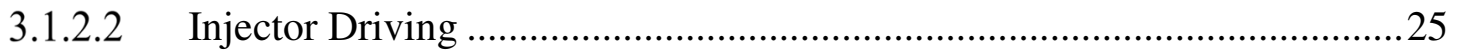

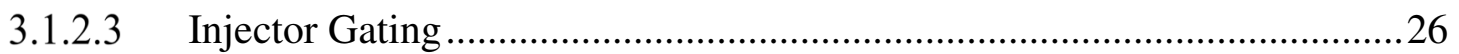

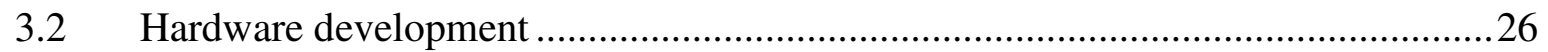

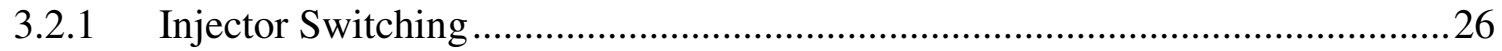

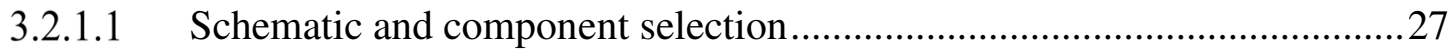

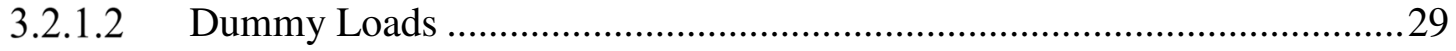

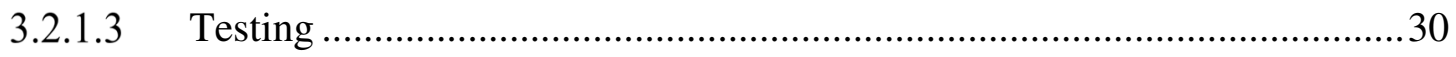

3.2.1.4 Proof of Concept.................................................................................

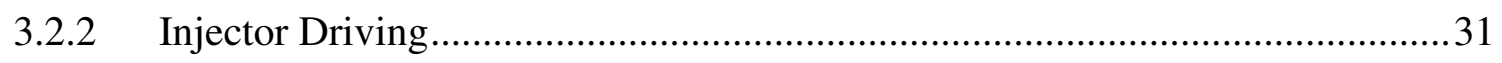

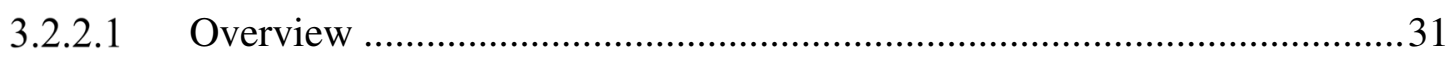

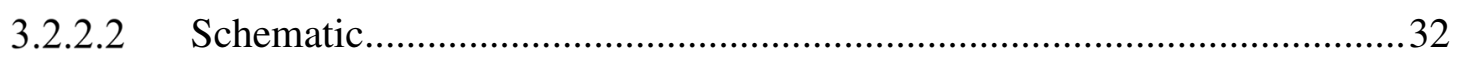

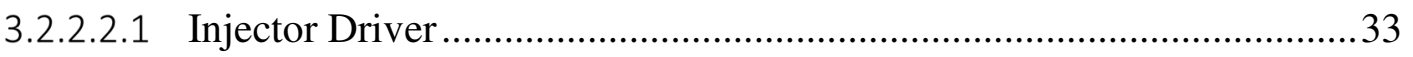

3.2.2.2.2 Switching Components and Current Monitor ....................................... 36

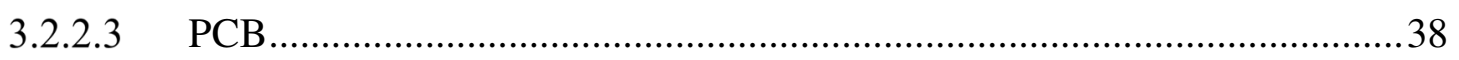

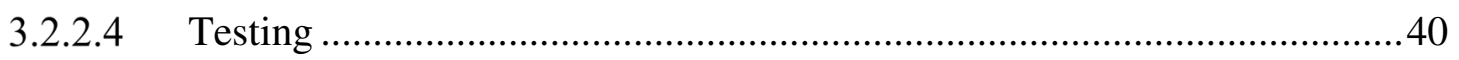

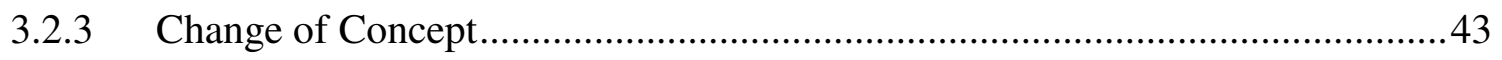

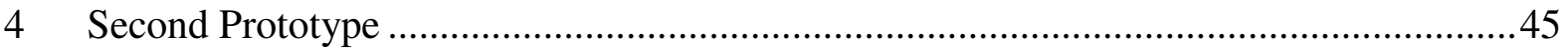

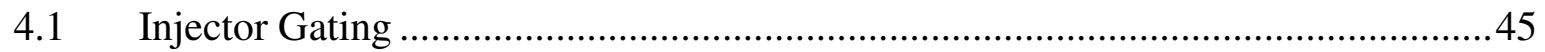

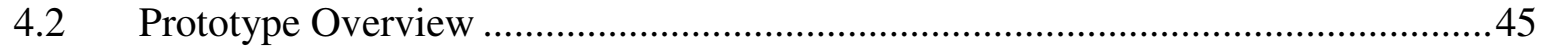




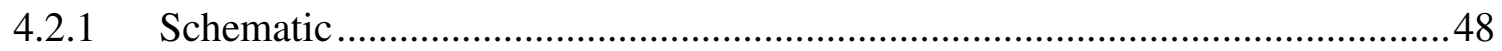

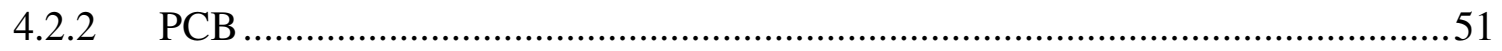

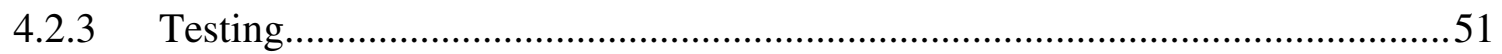

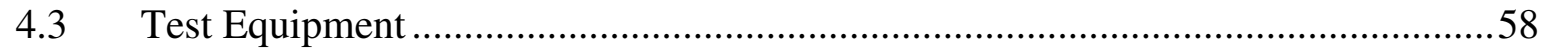

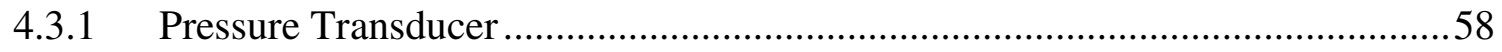

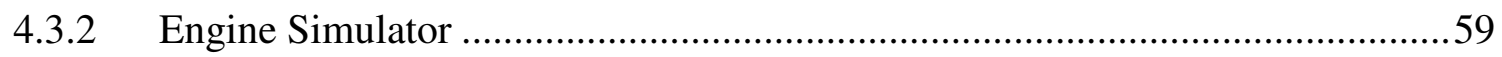

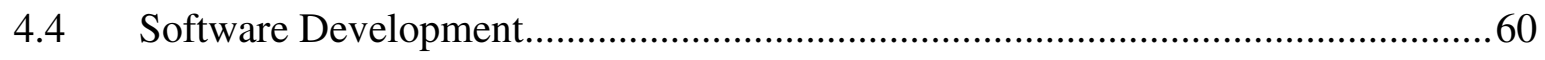

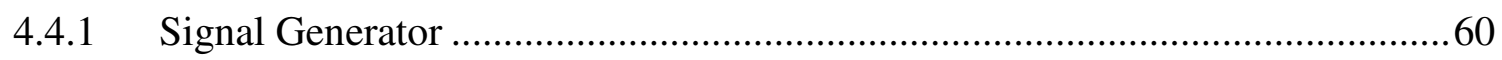

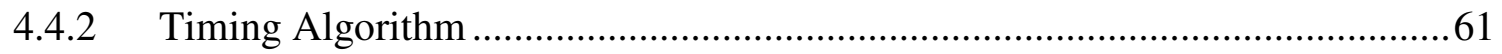

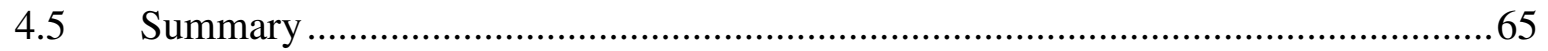

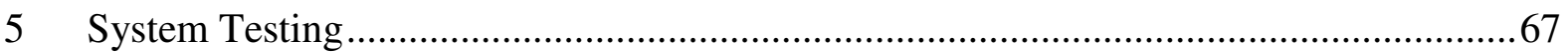

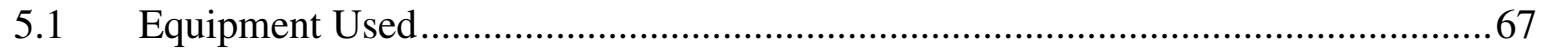

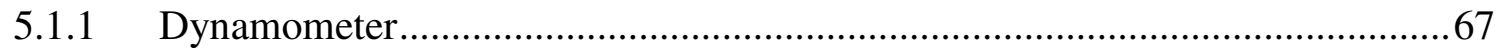

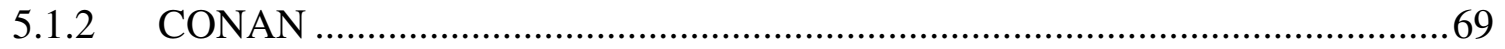

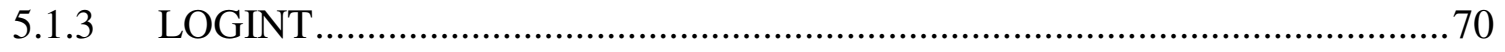

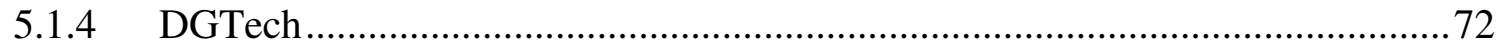

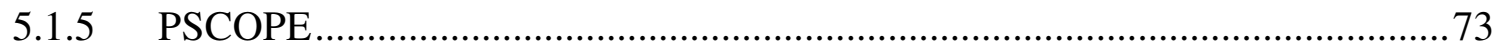

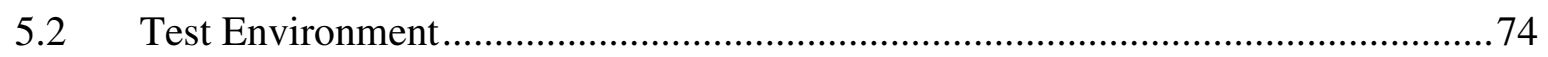

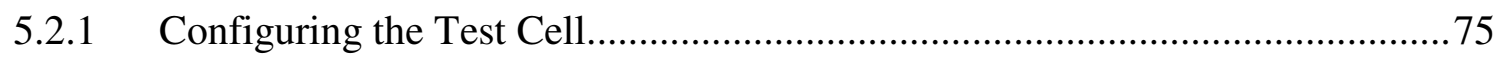

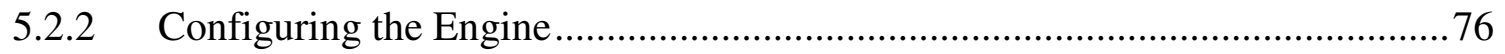

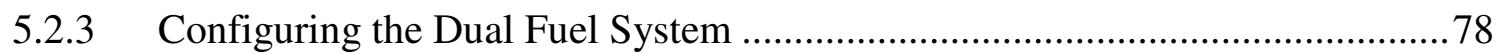

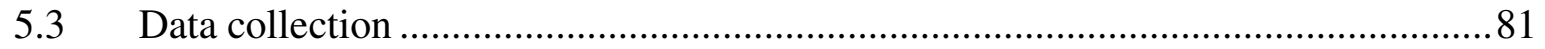

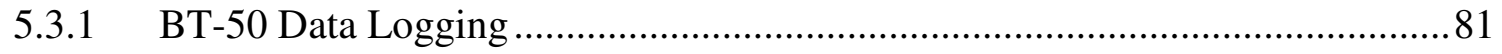

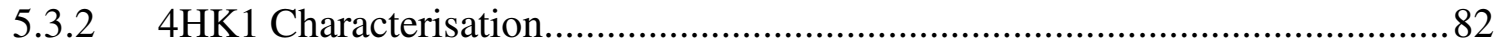

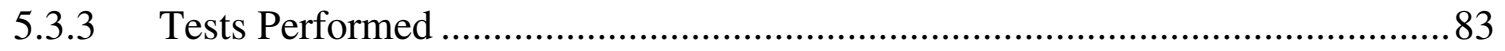

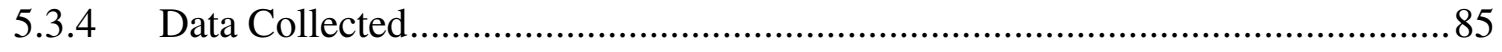

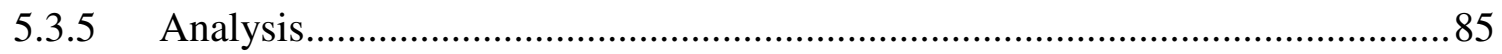


6 Results

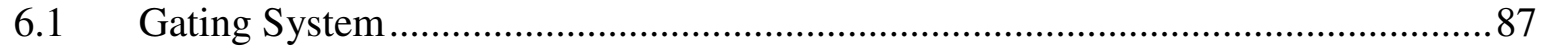

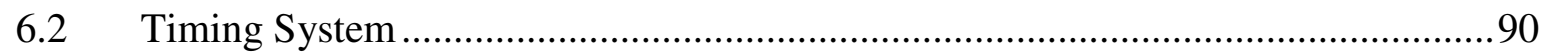

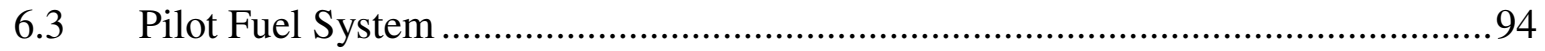

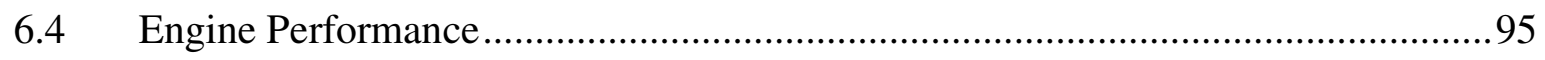

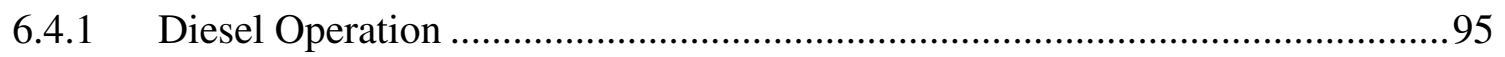

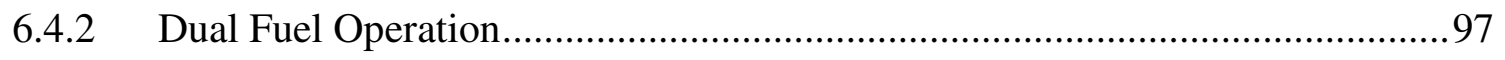

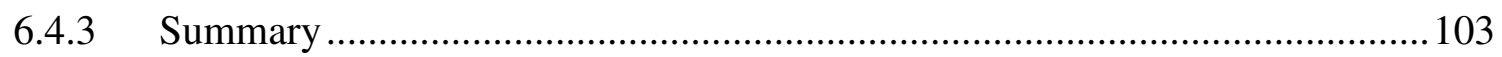

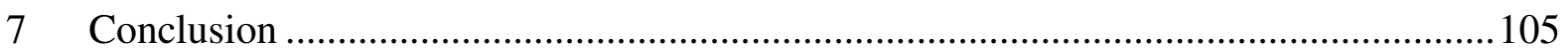

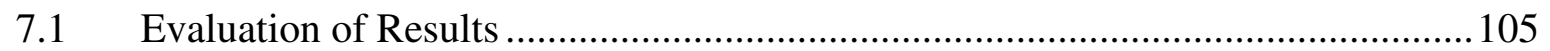

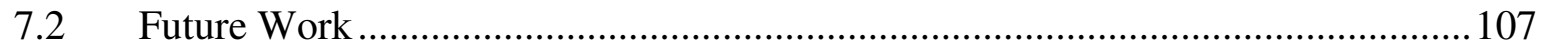

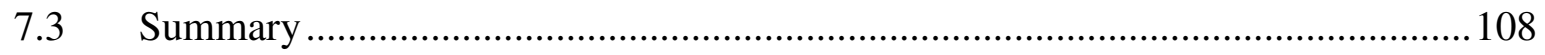

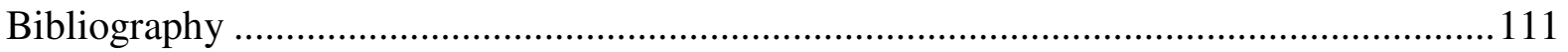

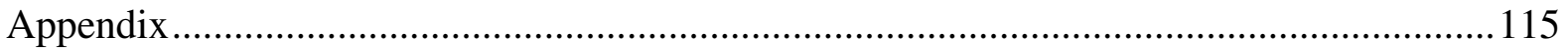

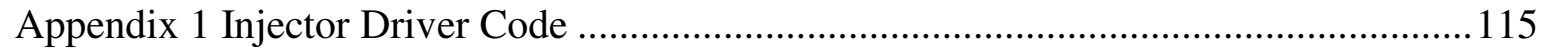

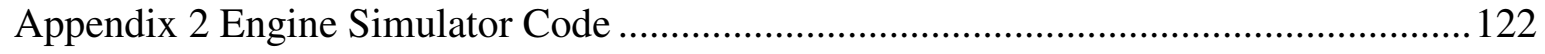

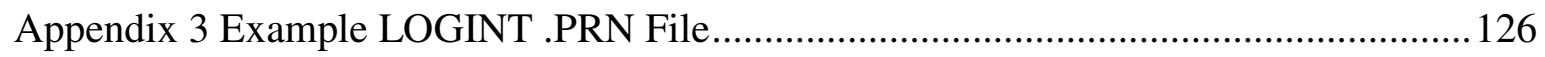

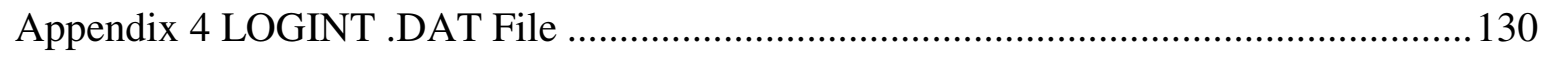

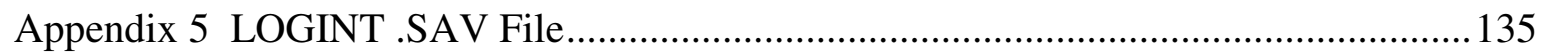




\section{List of Tables}

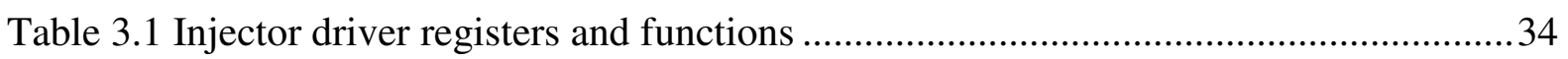

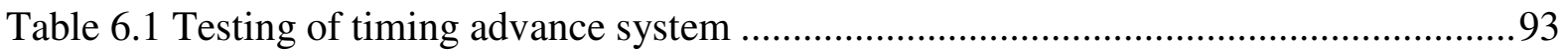

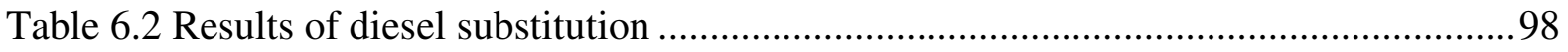




\section{List of Figures}

Figure 1.1 Dual fuel vehicle converted by DieselGas International [2] ................................ 1

Figure 2.1 Four stroke engine cycle [8] f.................................................................... 8

Figure 2.2 Cutaway of mechanical inline jerk pump [11] ...............................................

Figure 2.3 Common rail system schematic [12] ........................................................... 10

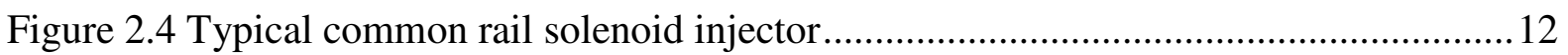

Figure 2.5 WL-C ECU input and outputs (reproduced with permission, Mazda Motor Europe $\mathrm{GmbH})$ 13

Figure 2.6 Mazda BT-50 utility vehicle. .14

Figure 2.7 WL-C CRDI Engine (reproduced with permission, Mazda Motor Europe GmbH)

Figure 2.8 Example of flywheel sensor output (reproduced with permission, Mazda Motor Europe $\mathrm{GmbH}$ ).

Figure 2.9 Example of cam shaft sensor output (reproduced with permission, Mazda Motor Europe $\mathrm{GmbH}$ ). 16

Figure 2.10 Oscilloscope traces of flywheel signal (green) and injector current (blue).........17

Figure 2.11 System diagram of auxiliary functions [17] ............................................... 18

Figure 2.12 Diagram of current DieselGas dual fuel system (reproduced with permission,

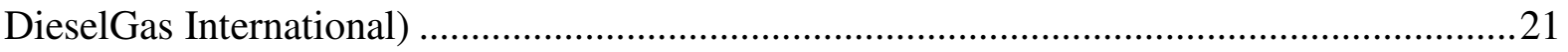

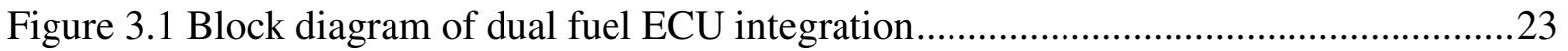

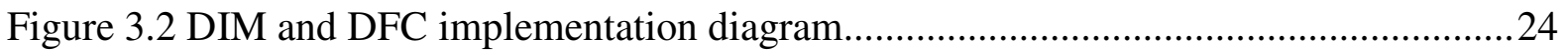

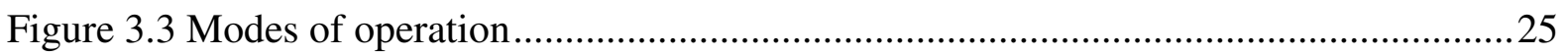

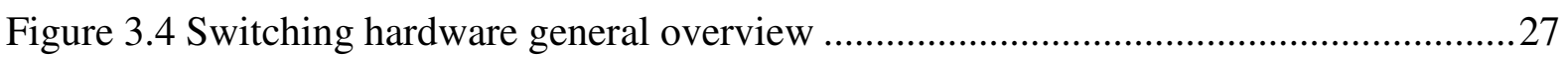

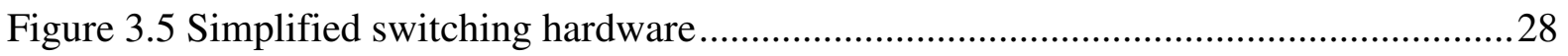

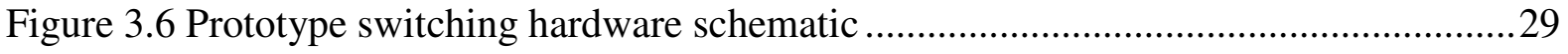

Figure 3.7 Prototype switching hardware plugged into BT-50 engine ..................................30

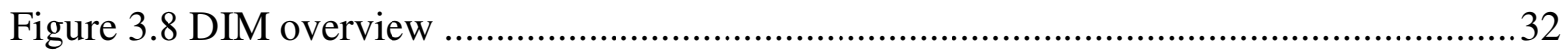

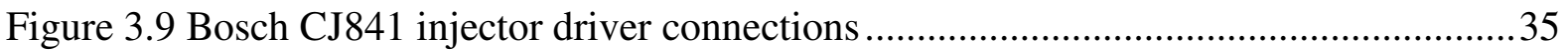

Figure 3.10 DIM proof of concept switching hardware ..................................................... 36

Figure 3.11 DIM proof of concept current sensing and signal conditioning ..........................37

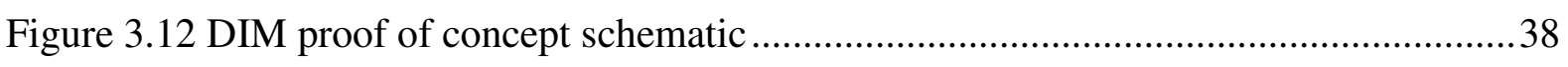


Figure 3.13 First DIM proof of concept PCB layout

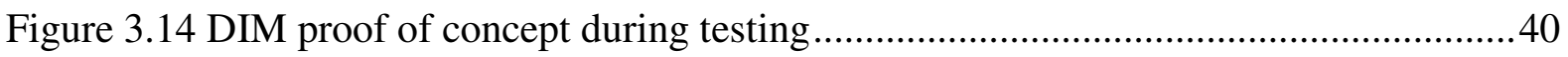

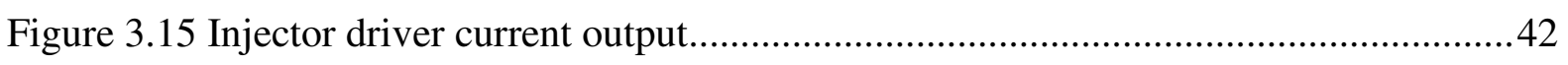

Figure 3.16 BOOST voltage (green) vs FET switching (purple)....................................... 42

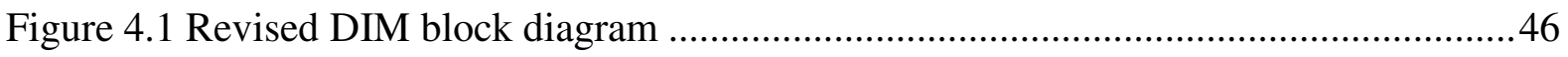

Figure 4.2 Detailed DIM block diagram showing DFC integration ....................................47

Figure 4.3 Revised switching hardware for injector gating and current detection .................49

Figure 4.4 Manufactured injector bobbin after powder coating ...........................................49

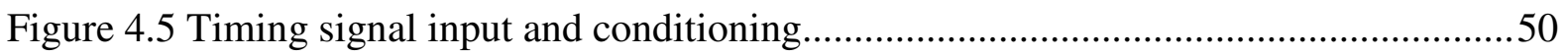

Figure 4.6 4HK1 engine cam shaft (blue) and flywheel (orange) sensor outputs ..................52

Figure 4.7 Cam shaft (phase) sensor waveform with (orange) and without (blue) sense

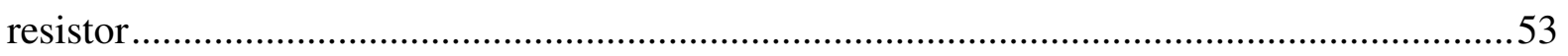

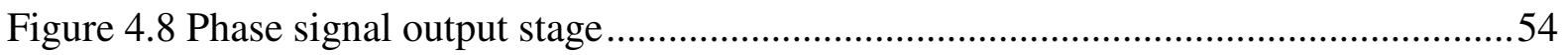

Figure 4.9 Phase signal input from engine and output to OEM ECU....................................54

Figure 4.10 Flywheel sensor before and after input stage (out of phase) ..............................55

Figure 4.11 Flywheel sensor before and after input stage (in phase) ..................................56

Figure 4.12 Shortened injection pulses (blue) slowing current tailing off slowly..................56

Figure 4.13 OEM injection pulses (blue) and reduced injection pulses from the DIM (orange)

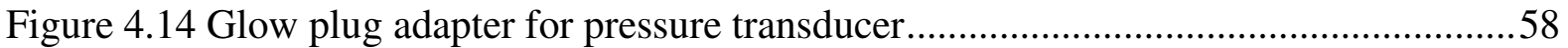

Figure 4.15 Pressure transducer installed in cylinder head................................................59

Figure 4.16 Comparison of engine (top) and engine simulator (bottom) signals, post-signal conditioning

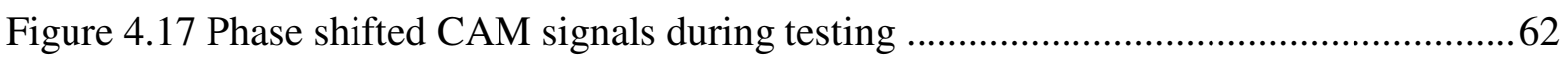

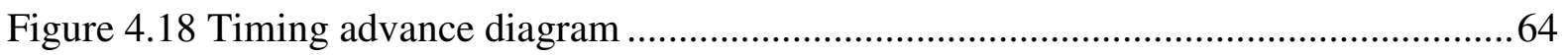

Figure 5.1 Diagram of water-brake dynamometer [35] ...................................................67

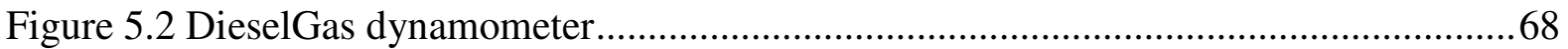

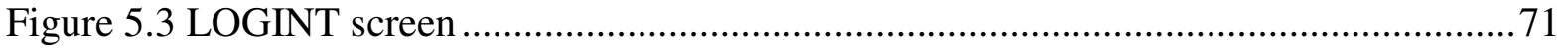

Figure 5.4 Rail pressure sensor and output characteristics [36] ..........................................72

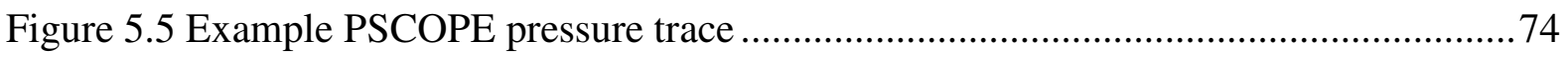

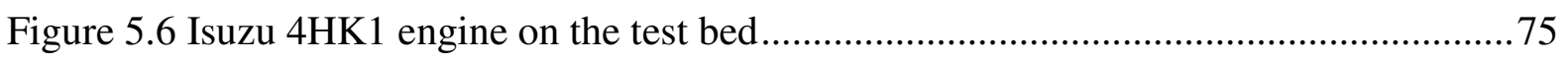

Figure 5.7 Injectors removed from engine. Note carbon build-up on \#1 ..............................77

Figure 5.8 Throttle body and gas valve installed in air intake ............................................. 78

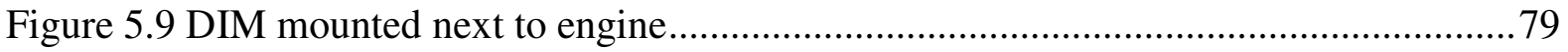




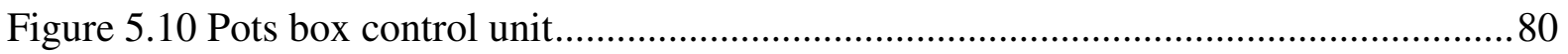

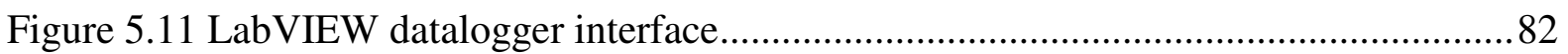

Figure 5.12 Example lug curve taken from 4HK engine ................................................... 83

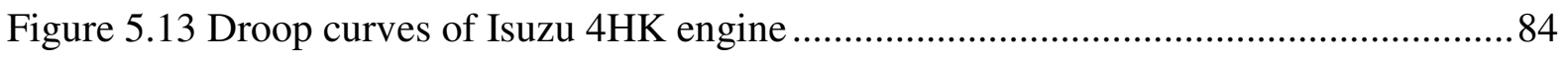

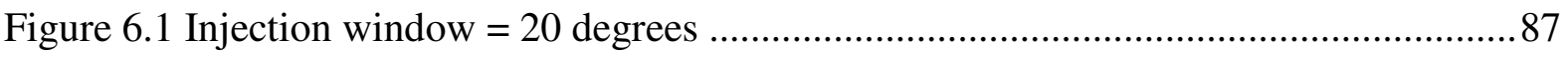

Figure 6.2 PSCOPE trace of engine running on diesel, both injections allowed.....................88

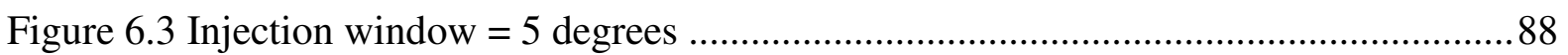

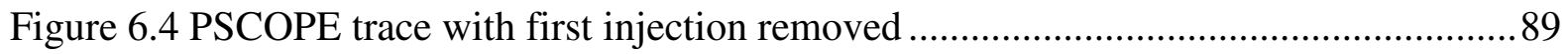

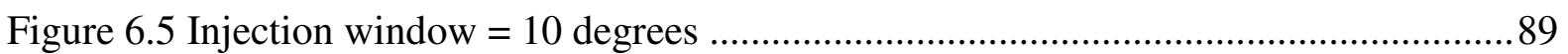

Figure 6.6 Comparison of flywheel sensor input (orange) and DIM output (blue) ................90

Figure 6.7 Measurement of timing delay on flywheel sensor input/output ...........................91

Figure 6.8 Comparison of cam sensor input (orange) and output (blue) ...............................92

Figure 6.9 First pulse removed, timing advanced 6 degrees............................................93

Figure 6.10 Pilot diesel pulse (orange) and injection window (blue) ..................................95

Figure 6.11 Maximum torque curve (lug curve) of Isuzu 4HK running on diesel ................95

Figure 6.12 Isuzu 4HK droop curves showing governor performance with varied $\%$ load ....96

Figure 6.13 Diesel flow and gas substitution vs injection duration at $1600 \mathrm{rpm}$....................99

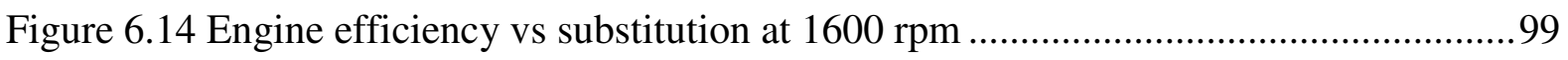

Figure 6.15 Dual fuel flow and torque with increased substitution at 1400rpm................... 100

Figure 6.16 Pressure trace during dual fuel operation ...................................................... 100

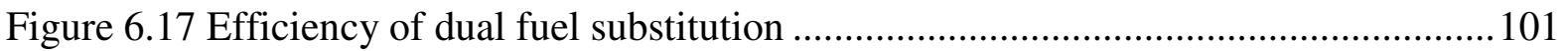

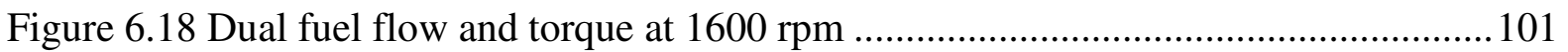

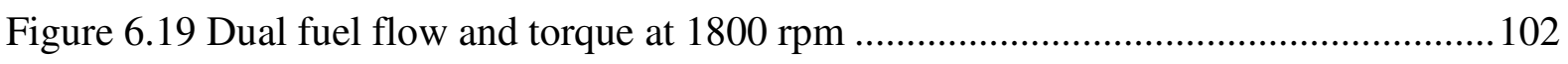




\section{Glossary}

APP Accelerator Pedal Position

BARO Barometric Pressure

CAN Controller Area Network

CKP Crank shaft Position

CMP Cam shaft Position

CNG Compressed Natural Gas

CRDI Common Rail Direct Injection

DFC Dual Fuel Controller

DGC DieselGas Controller

DIM Diesel Injection Module

ECU Electronic Control Unit or Engine Control Unit

EGR Exhaust Gas Recirculation

FET Field Effect Transistor

IAT Intake air temperature

IGBT Insulated Gate Bipolar Transistor

IMAP/MAP Intake Manifold Air Pressure

IMAT/MAT Intake Manifold Air Temperature

LNG Liquefied Natural Gas

LPG Liquefied Petroleum Gas

MAF Mass Air Flow

MIL Malfunction Indicator Lamp

$\mathrm{NO}_{\mathrm{x}} \quad$ Nitrogen Oxides

OBD On-Board Diagnostics

OEM Original Equipment Manufacturer

PCB Printed Circuit Board

PID Proportional-Integral-Derivative control system

RPM Revolutions Per Minute

TDC Top Dead Centre

VBC Variable Boost Control

VSC Variable Swirl Control 


\section{Introduction}

\subsection{Overview/Motivation}

The motivation for the development of this project is both economical and environmental. With a large focus on efficient and eco-friendly transportation, alternative fuels have become an attractive option for vehicle owners. With the recent drop in natural gas prices and a continued rise in oil prices, natural gas has become an attractive alternative fuel source for diesel truck owners. By substituting up to $90 \%$ of diesel with cheaper natural gas, the cost of fuel is reduced significantly, while also reducing the amount of greenhouse gases and other harmful emissions released into the environment [1].

By using the otherwise underutilised fuel source that is natural gas, we are making full use of the planet's limited fossil fuel reserves, and reducing harmful emissions, while still powering the trucks and machinery required to deliver the standard of living to which we have grown accustomed. Until alternative renewable fuel sources become readily available, the world is still reliant on liquid fossil fuels (e.g. petrol and diesel) as the primary energy source for transportation of goods. Natural gas offers a more environmentally friendly alternative to these liquid fuels, which can offset the consumption of heavier oils.

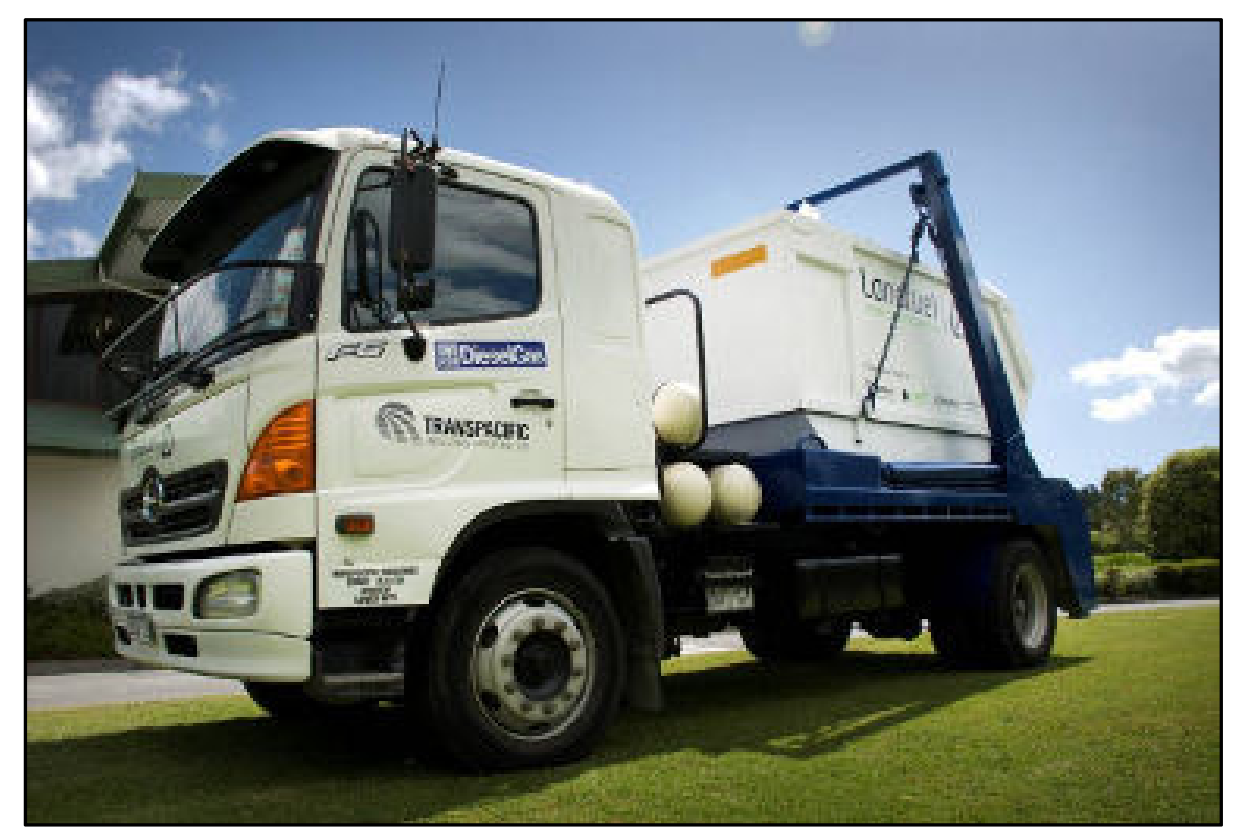

Figure 1.1 Dual fuel vehicle converted by DieselGas International [2] 
Dual fuel engines provide a solution to this problem. By taking an existing diesel engine, and using natural gas as the primary fuel source, it is possible to reduce oil consumption and emissions production, while maintaining the same power output, and at lower overall fuel cost. Currently, only a few engine manufacturers have taken up active research and development of dual fuel specific engines, which has created an opportunity for third party dual fuel conversions.

DieselGas International are a local alternative fuels company who develop dual fuel conversion kits for high speed diesel engines, such as those commonly found in highway trucks and stationary generator sets. DieselGas' primary product is a dual fuel kit which can be installed on engines utilising Bosch mechanical fuel pumps (an example of which is the Hino J08C engine used to power the Hino truck shown in figure 1.1). This limitation to a single specific (albeit common) fuel pump comes from the requirement of specific actuation hardware designed to fit this pump.

With the introduction of modern electronically controlled common rail fuel systems, it is possible to design dual fuel kits which can control the fuelling with no mechanical modification to the engine itself. The purpose of this project is to design a new electronic control unit (ECU) which can be used on any common rail diesel engine using solenoid injectors, regardless of manufacturer, and provide the dynamic fuel control for which the DieselGas product is known. This dual fuel ECU will be a novel addition to the DieselGas range of products, and will be required to meet the same specifications as the existing DieselGas dual fuel kits (described in the next section) in terms of fuel control.

It is important to ensure that the dual fuel ECU designed allows the engine to still operate within the manufacturer's original specifications to deliver a comparable power output, and not exceed the emissions requirements. In order for this project to advance into production, the dual fuel conversions must ensure that the engines meet the specific emissions requirements for the target market (for example Euro III in Europe or Tier 2 in USA) [3].

\subsection{General Background}

Dual fuel engines have been in development for several decades now, with advances in control technology making them more efficient and economically viable. Electronic control allows various engine parameters to be changed dynamically while the engine is running. Various control systems and algorithms found in modern ECUs are now used to fine-tune 
engine performance without any modification to the mechanical components of the engine [4].

Advances in diesel engine technology have made the diesel engine one of the most efficient internal combustion engines on the market today [5]. These advances have come in the form of improved manufacturing technology, better fuel quality, and improvements to the fuel delivery systems in the engines. Modern diesel engines have abandoned bulky mechanical injector pumps and now use common rail technology to control fuel delivery. Modern common rail fuel systems use electronically controlled fuel injectors and high pressure pumps to deliver precise volumes of fuel into the engine at precisely specified times to ensure the engine runs smoothly and efficiently.

Converting a diesel engine to dual fuel requires three main components:

1. A system to reduce the diesel volume delivered into the combustion chamber

2. A way to introduce the gaseous fuel into the combustion chamber to compensate for the reduced diesel

3. Software to control the specific ratios of the two fuels in the combustion chamber during engine operation

The design of these systems requires little to no modification to the internal structure of the engine, with the exception of the fuel pump (if installed). All other hardware is mounted externally. This is an important feature of dual fuel engines as it permits the operation of the engine in two modes: dual fuel mode or $100 \%$ diesel mode. The engine operator may choose to run the engine solely on diesel when circumstances require, such as low-power operation or when a gas source is not available. This makes dual fuel engines more versatile than an engine powered solely on diesel, and can increase the overall range of the vehicle.

Dual fuel kits designed by DieselGas International have been implemented on diesel engines with mechanical fuel systems (discussed in detail in section 2.3.1) and have been successfully sold in several countries. The proprietary dual fuel system developed by DieselGas was originally patented in 1983 and still forms the basis of current dual fuel system development today [6]. The heart of these kits is the supplementary electronic control unit which controls dual fuel delivery and monitors engine performance. With the commercial introduction of modern diesel engines with common rail fuel systems and electronic engine control, a new generation of ECU is required to interface with the existing electronic systems and perform 
the dual fuel delivery, while remaining invisible to the sensitive electronics of the manufacturer in order to maintain engine performance as close to the original specifications as possible.

\subsection{Thesis Objectives and Specifications}

The goal of this project is to design a generic dual fuel ECU for common rail diesel engines. The ECU must control the delivery of both gas and diesel, ignition timing, and all other associated engine parameters which require modification in order to operate on dual fuel.

After the dual fuel conversion, it should be possible to achieve greater than $50 \%$ diesel substitution with CNG. To match the performance of earlier DieselGas conversion kits, 70\% substitution should be possible, with a target maximum of up to $90 \%$ diesel substitution. The greater the diesel substitution, the more cost-effective it will be to implement on a commercial engine, and thus the more attractive the dual fuel system will be to a potential client.

The dual fuel ECU is designed to supplement the Original Equipment Manufacturer (OEM) ECU, not replace it. Therefore, the dual fuel ECU must be placed between the OEM ECU and the engine and modify the engine and OEM ECU signals in such a way that no intervention can be detected by the OEM ECU. This is important for two reasons: firstly, so that the OEM ECU does not erroneously detect any engine faults that might occur as a result of the dual fuel operation (which might result in the OEM ECU forcing the engine to shut down), and secondly because the dual fuel system is designed to operate within the manufacturer's original specifications. By matching the engine performance on dual fuel to that of the OEM specifications, the dual fuel conversion will not void the manufacturer's warranty.

The dual fuel ECU is to be designed in such a way that it can be applied to various common rail diesel engines, from 4 to 6 cylinders, with any capacity or power rating. By designing the dual fuel ECU to be generic, the only modification to the system required for each target engine will be an engine-specific fuel profile, with no modification to the system hardware from engine to engine. This will make the dual fuel ECU more versatile and lead to a shorter development cycle for implementation on subsequent engines. 
The final output from this project will be a generic ECU which will control the dual fuel operation of a common rail engine running on a test bed. This project uses an Isuzu common rail diesel engine taken from an industrial application as the test engine. The performance of the dual fuel system will be tested in a controlled environment and the validity of the system as a feasible dual fuel conversion method will be evaluated.

Engine performance across a variety of loads and speeds will be measured using a dynamometer and compared to the performance of the engine running purely on diesel. It is important that the dual fuel system be able to closely match the original engine output in terms of power and torque, as any significant power loss will not be tolerated by potential clients installing a dual fuel system on a commercial engine.

If time permits, the emissions profile of the engine will be measured across the same load characteristics and again compared to the operation on $100 \%$ diesel. Emissions standards apply to the specific year engines are manufactured, so an engine designed and built in 2013 will have to adhere to the emissions standards for that year. Therefore, it will be necessary to ensure that after a dual fuel conversion, the emissions profile does not exceed that of the emission requirements for each engine.

\subsection{Thesis Structure}

In chapter 2, the diesel engine is discussed in depth, from its inception by Rudolph Diesel, to the modern electronically controlled engines of today. The idea of dual fuel engine conversion is also introduced, showing how this offers advantages in fuel savings and reduced environmental impact. Lastly, the work of DieselGas International is discussed, describing their philosophy of bringing fuel savings to their customers while getting the best performance out of their engines through their unique technology systems.

Chapter 3 describes the initial phases of the project, from specification and concepts, through to the first rudimentary subsystem production and testing on a small engine in a light utility vehicle. The development and bench testing of the first prototype is described in this chapter, along with the reasons for the eventual abandonment of this design.

Chapter 4 details the development of the second prototype, and describes why this is a safer and more practical solution to the problem. The chapter details the schematics of the various subsystems, and production of custom electrical and mechanical components. This second 
prototype is then tested on a donated industrial engine, which first required mounting to the test bed and dynamometer, as well as the attachment of the requisite facilities for air, fuel, and electrical signals.

Chapter 5 outlines the testing of the various system components and engine performance. This chapter discussed the equipment used, the testing methodology and data acquisition techniques, and how the dual fuel system performance is measured.

Chapter 6 discusses the results of the engine tests performed in chapter 5. The testing of the individual subsystems is performed before the results of the engine running on dual fuel are compared to the engine running on diesel.

Chapter 7 concludes the thesis and evaluates the results obtained in Chapter 6 by discussing the advantages and shortcomings of the prototype, and how future improvements can enhance the performance of the system. 


\section{Background}

\subsection{The Diesel Engine}

This chapter introduces the technology used by modern diesel engines, detailing the advances that have led to the creation of the Common Rail Direct Injection (CRDI) system. These advances have been largely driven by economic and environmental factors, producing engines that consume less fuel while producing fewer harmful emissions.

\subsubsection{History}

The diesel engine was developed in 1895 by Rudolph Diesel. Initially designed to run on coal dust (an otherwise unused fuel source at the time) Diesel's engine was converted to oil after experiments with running on coal dust led to failures and explosions [7].

Since then, the development of diesel engines has progressed rapidly though advances in mechanical design and manufacturing technologies. Diesel engines are used to power heavy machinery such as ships, trains, trucks, and more recently, cars. With the advent of electronic engine control, modern diesel engines have become some of the most efficient internal combustion engines on the market today [5].

Modern diesel engines found in today's trucks and cars are 4-stroke internal combustion engines that rely on the same principles as petrol engines. Four stroke engines have four stages (or strokes) corresponding to the four up or down movements of the piston per engine cycle. The four strokes (as shown in figure 2.1) are:

1. Intake: The piston moves down drawing fresh air into the cylinder which is mixed with fuel

2. Compression: the piston moves up and compresses the air/fuel mixture

3. Power: shortly after the piston reaches the top of its travel, the air/fuel mixture is ignited, forcing the piston down

4. Exhaust: on the way back up, the piston pushes the exhaust gases out of the cylinder 


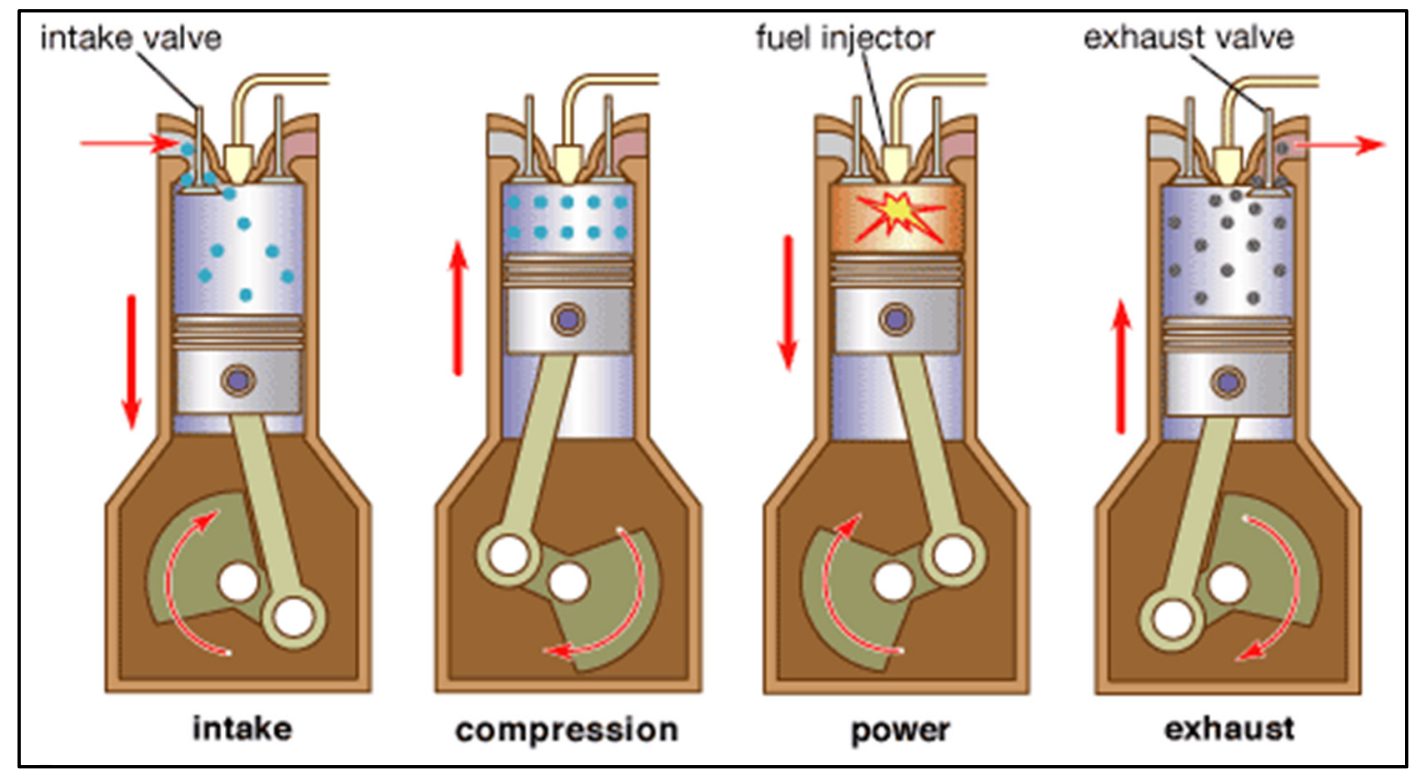

Figure 2.1 Four stroke engine cycle [8]

In petrol engines, the fuel is mixed with air before it enters the intake port, producing what is known as a homogeneous charge. Ignition is provided by a spark plug (as seen in figure 2.1) which ignites the air/fuel mixture, which leads to the label of a Homogeneous Charge Spark Ignition (HCSI) engine. In diesel engines fuel ignites under the heat of compression, and thus they do not require spark plugs. The fuel is also delivered directly into the combustion chamber, in what is known as a stratified charge [9]. For this reason, diesel engines are given the label Stratified Charge Compression Ignition (SCCI) engines. Because of the high pressures involved in operating a diesel engine (both in the fuel system and cylinders) diesel engines must be designed to handle higher stresses and temperatures than those found in a petrol engine of the same size. Because of these more exacting manufacturing requirements, diesel engines are typically more costly to manufacture than their gasoline counterparts, but offer greater power output and efficiency, lower maintenance, and longer life cycles [10].

The most important component of the diesel engine is the fuel system. The fuel system is responsible for making sure the correct quantity of fuel required is delivered, and at the right time. The injection method also ensures the fuel is adequately atomised and mixed with the air in the cylinder to ensure sufficient mixing of air and fuel to achieve complete combustion. These processes are referred to as fuel metering, timing, atomisation, and mixing.

Because the performance of diesel engines is so reliant on that of the fuel system, many advances in diesel engine technology have come from improvements in the various fuel system components. The basic premise of diesel injection systems is to inject a metered 
quantity of fuel into the cylinder under sufficiently high pressure to atomise the fuel such that it will readily combust under the heat of compression. There have been several advancements in the design of fuel injection systems, the majority of which were based on a cam shaft driving a pumping element to deliver pressurised fuel to an injector. Early metering systems were mechanically driven, often by way of a control rack connected to a mechanical governor. One of the most common mechanical injection systems in use prior to the adoption of the modern common rail system is the jerk pump.

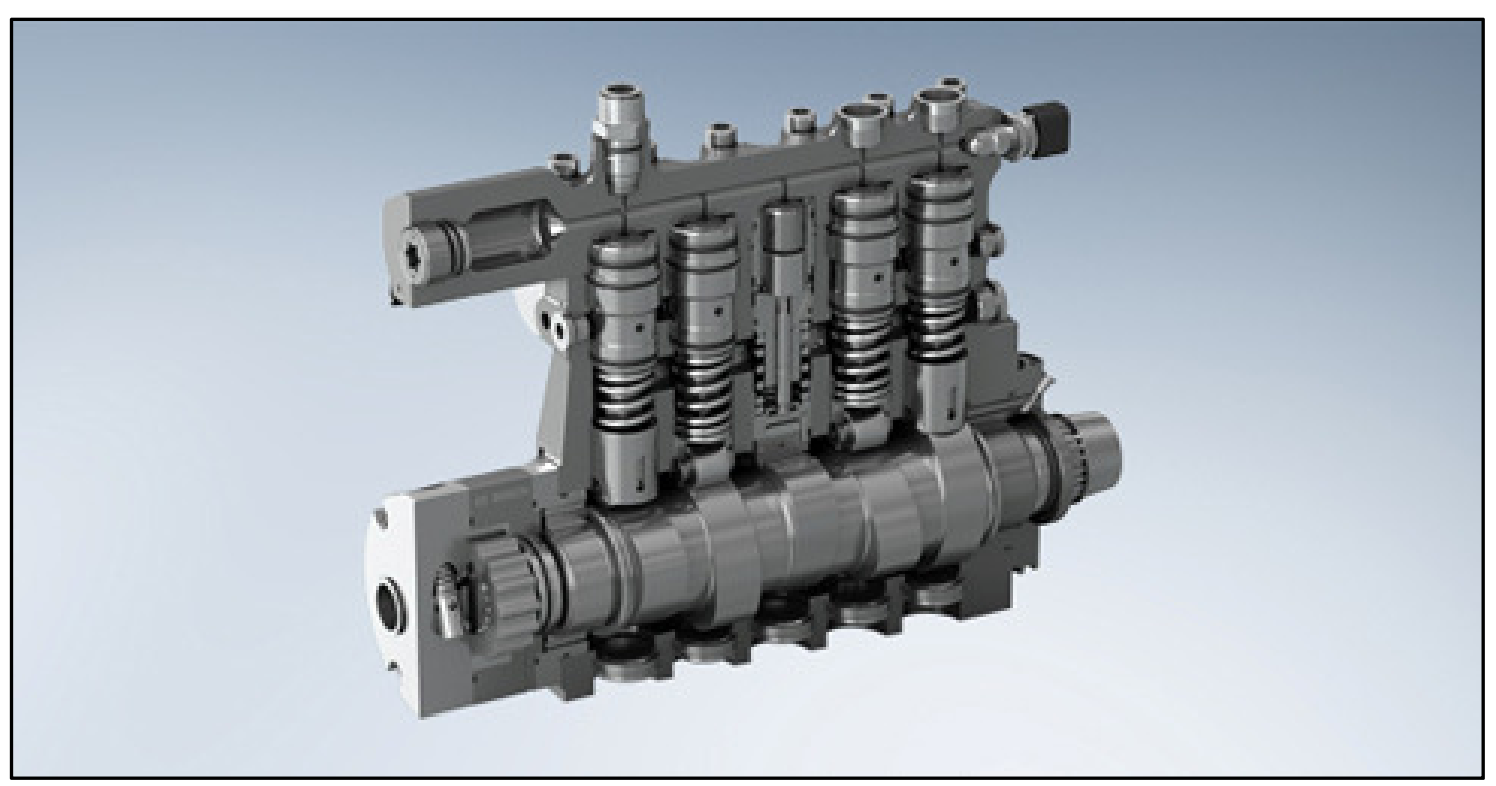

Figure 2.2 Cutaway of mechanical inline jerk pump [11]

A jerk pump is an in-line type fuel injection pump consisting of several plungers, one for each engine cylinder (figure 2.2). These plungers are driven by a cam shaft to deliver a metered amount of fuel to the injectors. The plungers are cut with a helical shaped groove which, when rotated within the pump chamber, exposes a spill port at different points in their travel. This allows the amount of fuel sent to the injector to be varied, as when the spill port is exposed, fuel pressure in the pipeline drops and the return spring in the injector closes the needle valve thus ending injection. The helical plungers are all rotated by a common rack gear driven by the engine governor which determines the amount of fuel required as well as the start of injection.

There are several other mechanical fuel delivery systems in operation today, and most operate on these same basic principles. In more modern engines, the injectors may be electronically activated, allowing higher pressures to be reached, and to more accurately meter the amount of fuel injected. These advances have led to more efficient, more powerful and also quieter 
engines. With the introduction of electronically controlled injectors, an electronic control unit is required (ECU) to monitor the engine's state and open and close the injectors at the correct time.

\subsubsection{Common Rail Technology}

The common rail fuel system (figure 2.3) consists of four main components:

- High pressure fuel pump

- Accumulator (common rail)

- Injectors

- $\mathrm{ECU}$

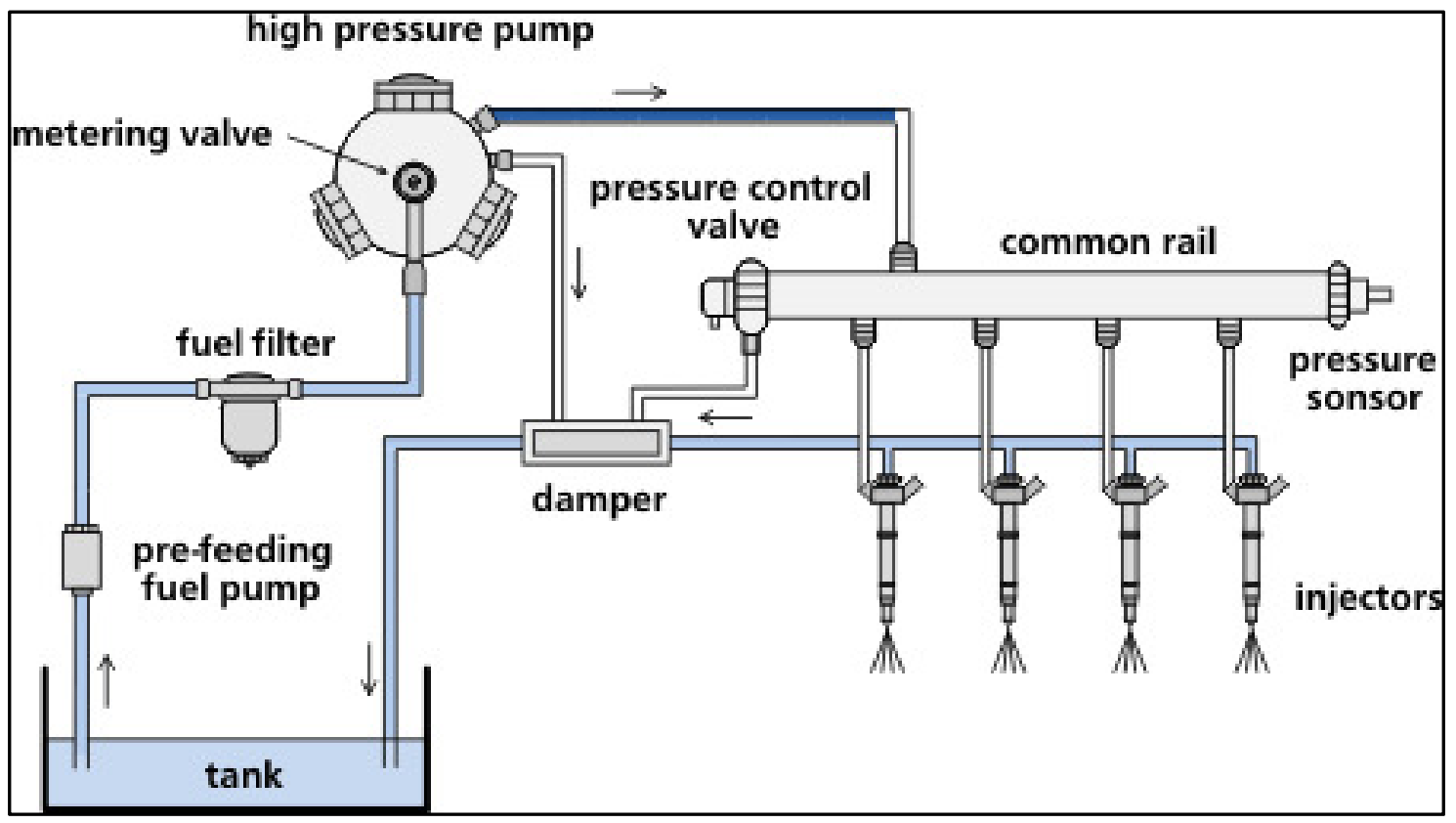

Figure 2.3 Common rail system schematic [12]

\subsubsection{Fuel pump}

The high pressure fuel pump used in common rail systems pressurises the fuel to a much higher pressure (up to $300 \mathrm{MPa}$ ) than a conventional fuel pump (up to $200 \mathrm{MPa}$ ). The fuel pump in a CRDI engine is driven from the flywheel and is often a lobed-style pump. The amount of fuel pressurised by the pump is controlled by a metering valve which limits the fuel uptake of the pump. This valve consists of a solenoid driven by the ECU, which lifts a needle valve on the intake of the pump, thus controlling the amount of fuel available to pressurise the accumulator. 


\subsubsection{Accumulator}

The fuel pump pressurises the fuel inside an accumulator, also known as a common rail. The accumulator contains pressurised fuel for the injectors. The accumulator is the critical component of a CRDI engine and serves three main purposes: firstly, the accumulator holds fuel at a specific pressure (up to $300 \mathrm{MPa}$ ), ensuring all injectors can deliver fuel under the same pressure, making calculation and calibration of fuel delivery more precise; secondly, the accumulator, with its large volume of fuel, is able to absorb some of the rapid pressure fluctuations in the system caused by the pumping and injection operations; thirdly, by using a common rail system to feed all injectors, the overall complexity of the system is reduced by eliminating the need for bulky mechanical pumps for each injector. This means that the injectors are not subjected to large changes in pressure (only approx. $4 \mathrm{MPa}$ peak to peak) throughout their operation. Overall, this makes the fuel injection events more uniform across all injectors/cylinders and results in a smoother running engine [13].

\subsubsection{Injectors}

The high-pressure injectors in a CRDI engine are electronically-controlled valves that deliver fuel into the cylinders of the engine. Because the fuel is administered directly into the cylinder, this is known as direct-injection. All modern commercial diesels are direct injection engines, and the injectors play an important role in delivering a fine mist of fuel through tiny ports in the tip of the injector. The more the fuel is atomised, the higher surface-area to volume ratio, and the more efficiently it will burn. Common rail engines produce very efficient engines because the high fuel pressures used lead to better fuel atomisation [14].

The injectors found in modern common rail diesel engines are of two types; solenoid or piezo actuated. Solenoid injectors (such as the one seen in figure 2.4) are the most common and are controlled by sending current through a coil of wire (solenoid) and inducing a magnetic field to lift a needle valve. Piezoelectric injectors use the piezoelectric effect of crystals changing in thickness under an applied voltage, and employing this change in dimension to lift the valve. Solenoid valves are currently more common than piezoelectric injectors mainly because of their lower cost and have been in production for decades [15]. However, piezoelectric valves are becoming more common as costs come down, as they do offer faster response times and better atomisation characteristics than traditional solenoid injectors [16]. 


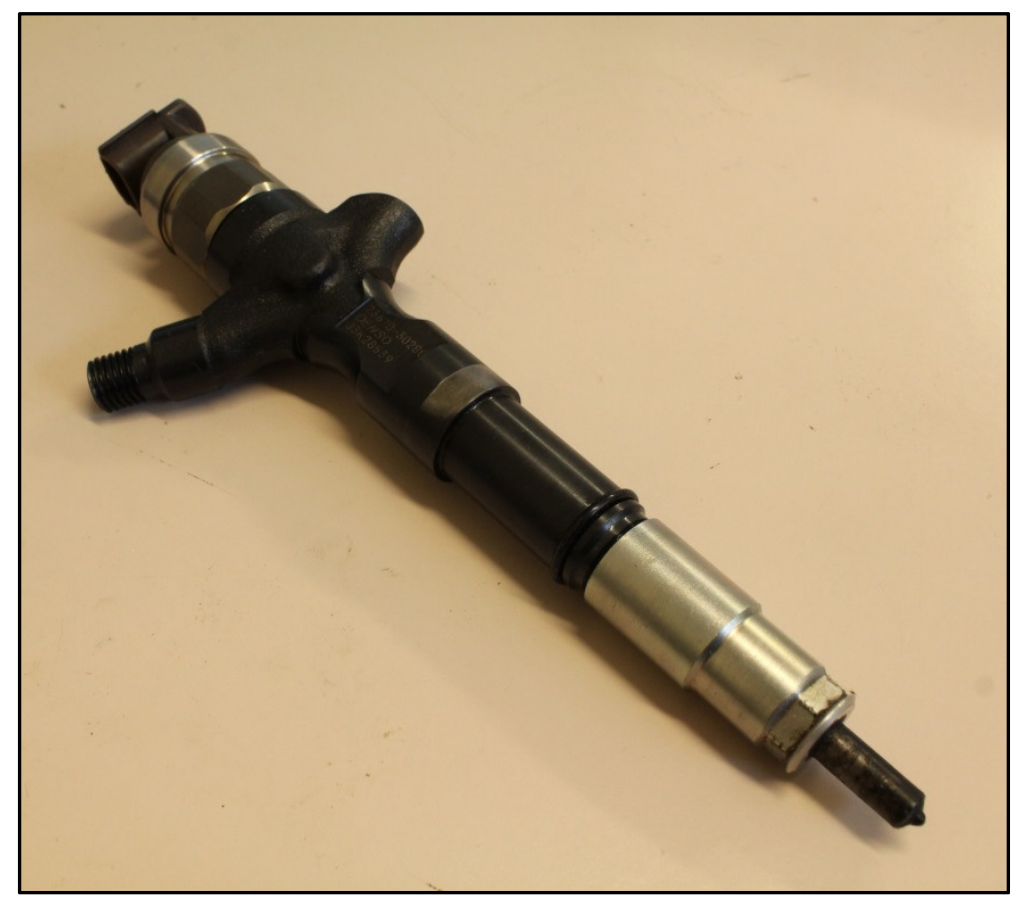

Figure 2.4 Typical common rail solenoid injector

Because diesel injectors are precise electromechanical devices, any variation in the manufacturing processes will lead to variance in the mechanical performance, and thus affect diesel delivery from injector to injector. The main parameters to be affected are opening time (the time delay between the injector current switching on and the needle valve opening), and fuel delivery (the total amount of fuel delivered per unit time). To reduce error in diesel injection amount, the OEM ECU sends a high current pulse to the injector to open it as fast as possible, and then switches to a lower holding current to keep it open. The injector also has a specific calibration code set in the factory, which contains a correction factor used by the OEM ECU to advance/delay the injector opening time and to increase/decrease the total on-time, to ensure a consistent fuel delivery for each cylinder.

\subsubsection{Electronic Control Unit}

The most important advance in engine fuel delivery systems is the introduction of electronics to monitor and control engine function. The electronically controlled injectors are operated by an engine control unit (ECU), sometimes referred to as a powertrain control module (PCM). The ECU takes information from a variety of sensors mounted on the engine and uses this data to control various engine parameters, resulting in an optimised, efficient, and quietly running engine. 


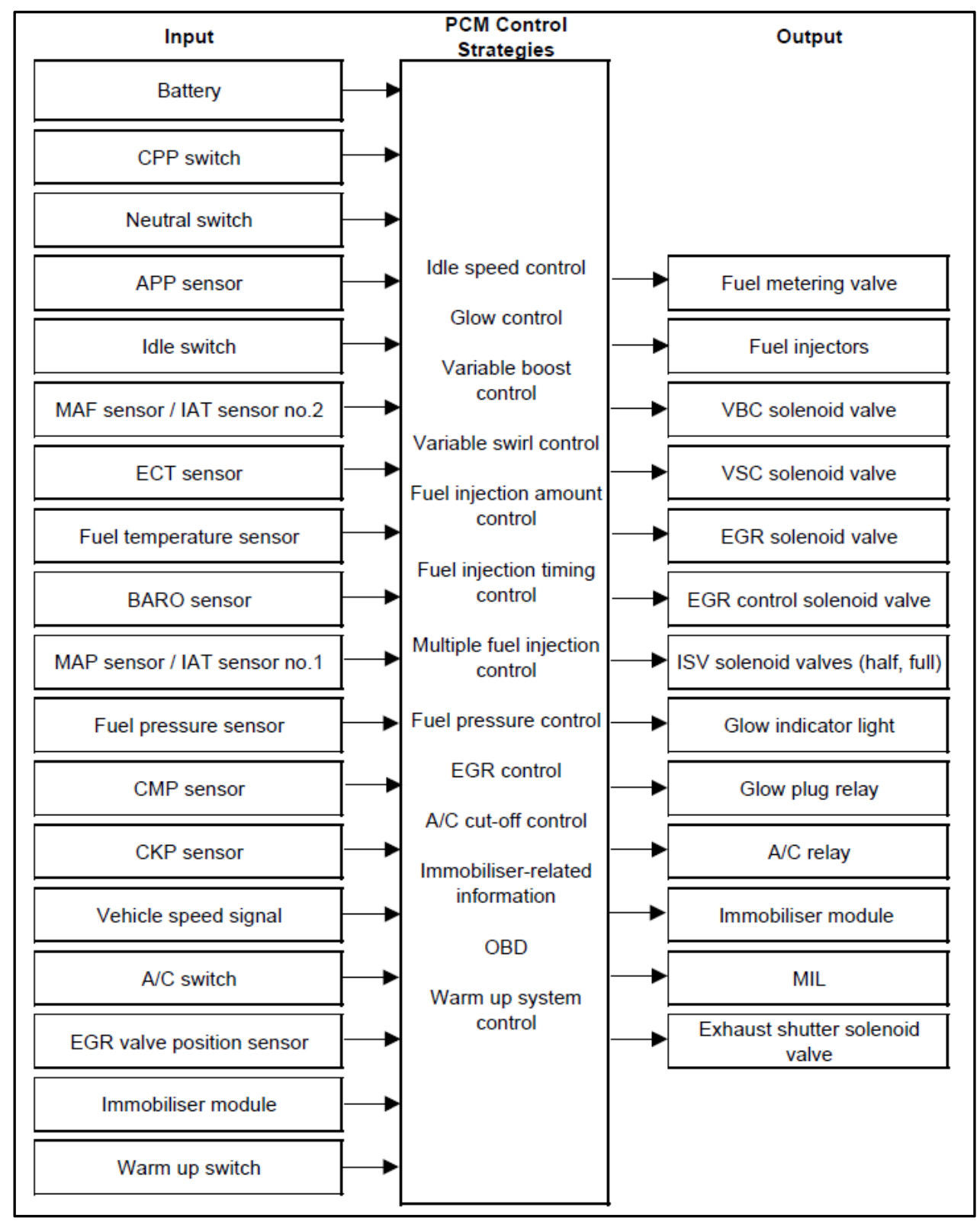

Figure 2.5 WL-C ECU input and outputs (reproduced with permission, Mazda Motor Europe GmbH)

Some of the primary sensors required by a CRDI engine are the timing sensors, used to detect the position and phase of the engine. These sensors use reference marks on the flywheel and cam shaft to determine where the engine is in its cycle. The timing sensors are crucial to the operation of the ECU, and without these, the engine will not run. The various sensors feed information to the ECU and through various algorithms, the ECU determines the correct time to open the injectors, and how long to hold them open for. The ECU has a wide variety of input and outputs, some of which are critical to engine operation, and others which control auxiliary vehicle functions. 
Some sensors of secondary importance to the operation of the engine include those measuring intake air temperatures and pressures, barometric pressures, exhaust gas temperature and oxygen levels. These are all sensors which are used to fine-tune the fuel delivery and performance of the engine in order to reduce emissions. An example of the various inputs and output of a typical ECU is shown in figure 2.5. The ECU is also used as a diagnostic tool, recording anomalous sensor values and detecting errors in performance. Modern ECUs can be interfaced with an external computer and the error codes recorded and engine parameters observed in real time. This makes servicing of modern engines much easier for technicians.

\subsection{Characterisation and Reverse Engineering}

Initial characterisation of a CRDI engine was performed on a Mazda BT-50 utility vehicle (figure 2.6) with a WL-C engine (figure 2.7). This initial characterisation helped create the initial designs and prove several concepts prior to the acquisition of the Isuzu 4HK1 engine used later in the project.

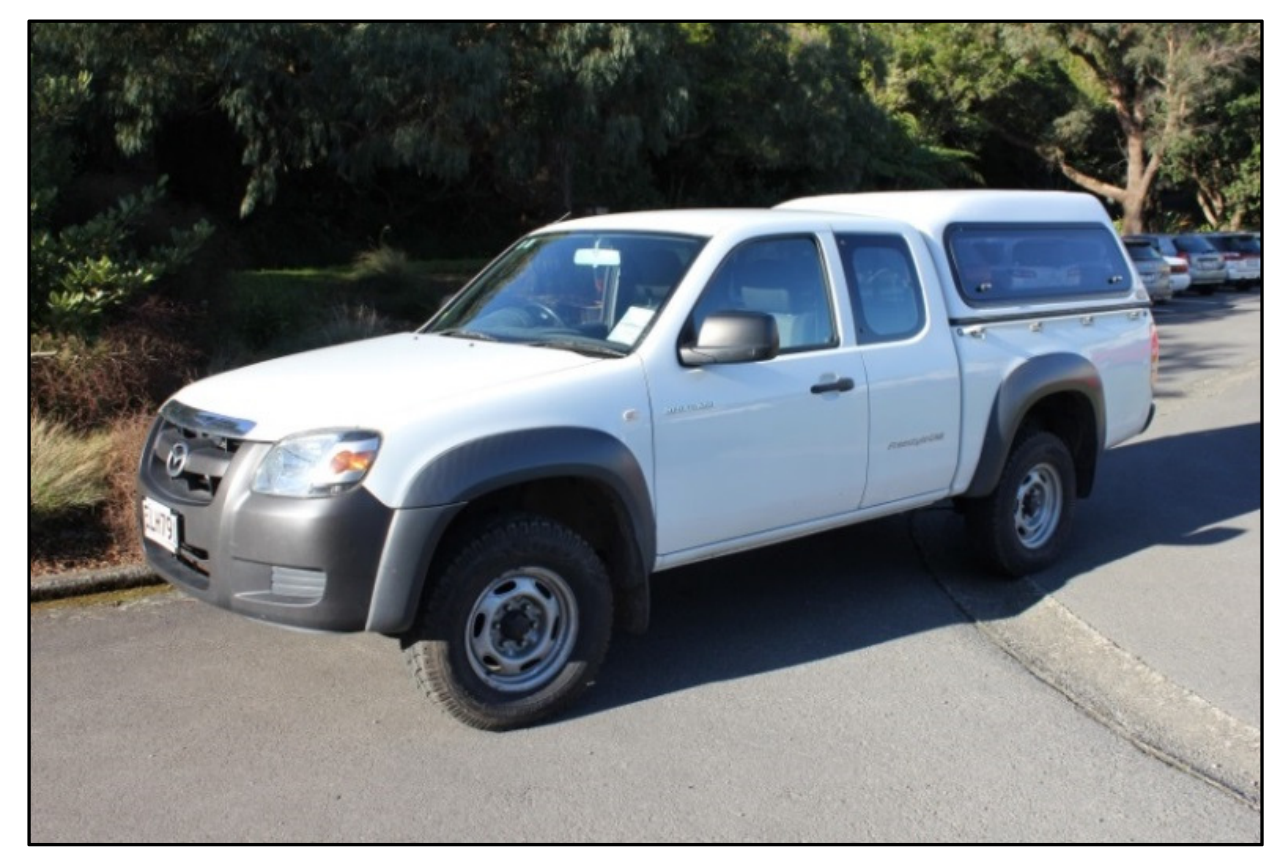

Figure 2.6 Mazda BT-50 utility vehicle

The WL-C engine is a 2.5 litre, 4-cylinder, turbocharged common rail diesel engine. This engine was analysed and monitored to gain a comprehensive understanding of the common rail system before development began on this project. It has all of the features characteristic of a CRDI engine as well as several other modern auxiliary features which, while not critical for operation, allow this engine to reduce emissions and increase efficiency. 


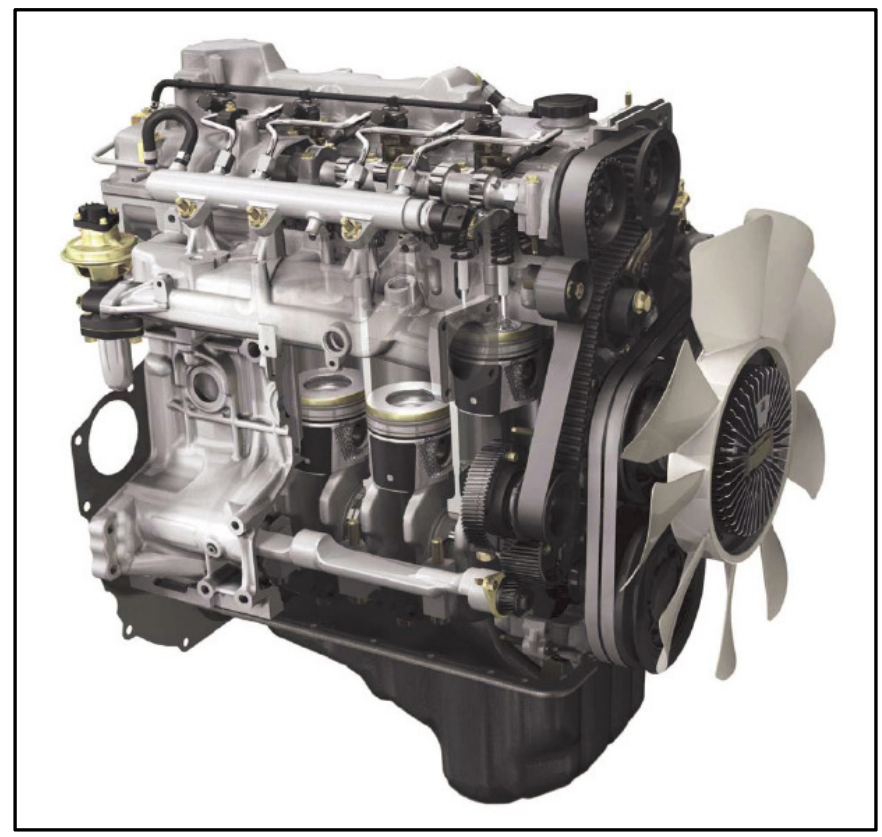

Figure 2.7 WL-C CRDI Engine (reproduced with permission, Mazda Motor Europe GmbH)

\subsubsection{Engine Timing Signals}

The timing signals for the WL-C engine come from the rotation of the flywheel and cam shaft. The flywheel is driven by the crankshaft, and the sensor that monitors its rotation is sometimes referred to as the crank or position sensor. The cam shaft sensor is referred to as the phase sensor. The position sensor tells the ECU where the crankshaft is in terms of its rotation. From this, the ECU can infer the position of the pistons. However, since a 4 stroke engine requires two revolutions of the crank per combustion cycle, it is impossible to tell from the position sensor alone what phase the engine is in (i.e. is the piston at the top of a compression or exhaust stroke?). Because the cam shaft spins at half the speed of the crankshaft, a sensor is used to detect the position of the cam shaft, thus allowing the ECU to determine what phase of operation the engine is in.

The BT-50 has a pulse wheel attached to the flywheel with 58 projections, spaced 6 degrees apart. There is an 18 degree gap where there are 2 projections missing which is used as a reference point for the ECU. The sensor is an inductive pickup mounted in the bell housing adjacent to the rotating flywheel and produces voltage spikes as the teeth of the flywheel rotate past it. The sensor produces positive and negative voltage spikes as the teeth approach and pass the inductive pickup which the ECU uses to determine position. The waveform of the WL-C crank position sensor output is shown in figure 2.8 below: 


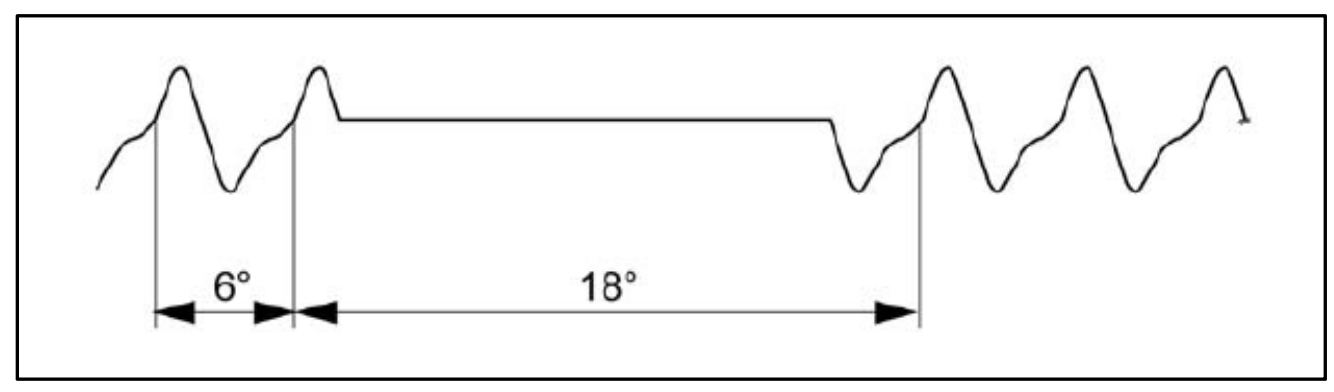

Figure 2.8 Example of flywheel sensor output (reproduced with permission, Mazda Motor Europe GmbH)

The phase sensor is a hall-effect sensor which produces a logic-level output when a metal tab on the cam shaft sprocket rotates past it. On the WL-C engine, there is a single signal pulse for the ECU to use as a reference point for the phase of the engine cycle (figure 2.9).

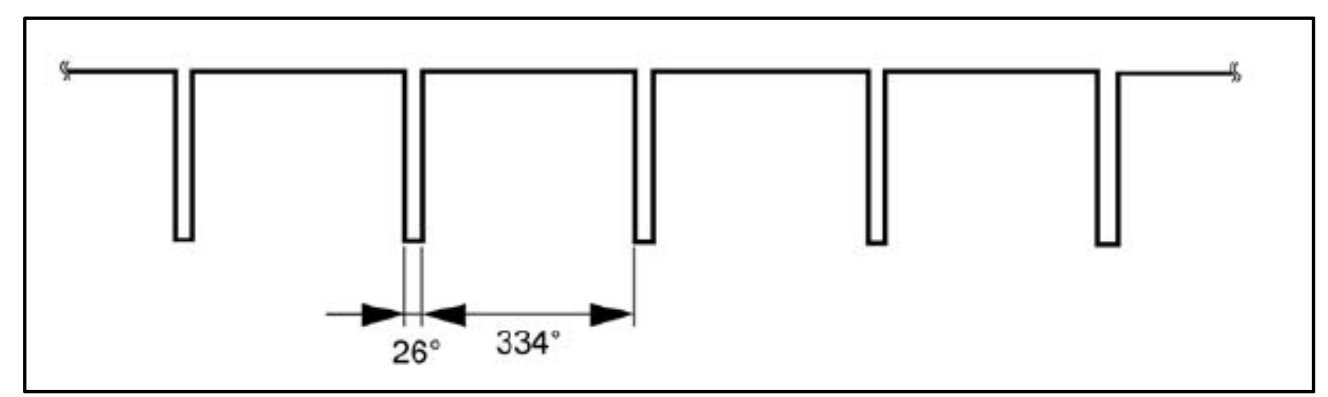

Figure 2.9 Example of cam shaft sensor output (reproduced with permission, Mazda Motor Europe GmbH)

The flywheel signal provides fine timing data to the ECU, allowing it to determine the optimal time to inject fuel into the engine. It also provides a way to monitor engine performance through measuring the time between adjacent projections, and inferring from this information the acceleration of the crankshaft. This allows the ECU to determine if the engine is speeding up or slowing down, or even if one of the cylinders is receiving more or less fuel than the others. The ECU uses all of this information to fine-tune the fuel delivery and ensure the engine runs smoothly, efficiently, and quietly.

\subsubsection{Diesel Injection Pulses}

A Tektronix current probe was placed around one of the injector lines on cylinder number 1 , and the injector current observed on an oscilloscope. At idle, there are pre and pilot injections of fuel before the main injection. However, at full load, only the main injection is present. A close up of the injection pattern seen in the WL-C engine is shown in figure 2.10. One can clearly see the pilot, pre and main injections (blue) and the flywheel timing signal (green) while the engine is at idle. 


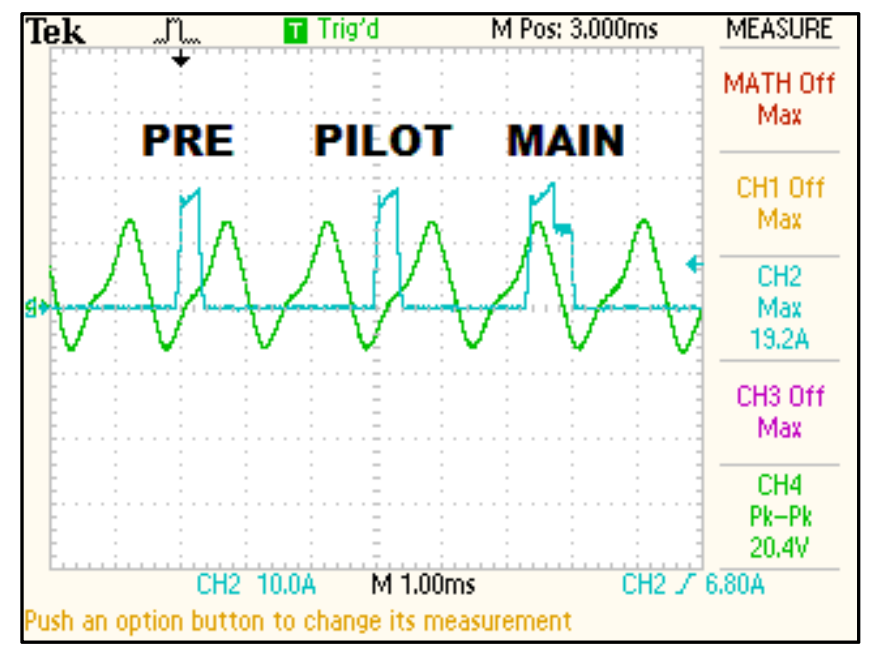

Figure 2.10 Oscilloscope traces of flywheel signal (green) and injector current (blue)

One of the main goals of this project is to modify the injection pattern above to alter the amount of diesel injected. As one can see from figure 2.10, the entire injection pattern occurs over approximately 30 degrees of engine rotation, and the total injector on-time at idle is approximately $2 \mathrm{~ms}$. To observe the injection pattern at different loads, a datalogger was connected to the engine. This is further described in section 5.3.1.

\subsubsection{On-Board Diagnostics}

The BT-50 is a modern vehicle which comes equipped with On-Board Diagnostic (OBD) capabilities. The OBD protocol is a standard protocol introduced in 1996 and is mandatory on all modern vehicles. The OBD system receives information from the ECU about engine performance and various parameters such as intake air pressures and temperatures as well as displaying any error codes generated by system faults.

It is critical that the dual fuel system does not disrupt the performance of the engine by producing error codes that may inhibit the operation of the OEM ECU. For example, the BT50 ECU can detect when an injector or sensor is disconnected, and can detect a misfiring cylinder. When an error code is detected, it is saved into memory and the check engine light is illuminated, prompting the operator to investigate the fault.

For the purposes of this project, a scan tool and associated software was acquired which interfaces with the OBD port located in the cab and allows the operator to check the engine fault codes, read engine parameters in real time, log engine performance and reset the ECU to clear logged fault codes. 


\subsubsection{Engine Auxiliary Functions}

The WL-C engine has several auxiliary functions designed to increase engine efficiency and reduce emissions. These functions can be seen in figure 2.11 and include:

- Variable swirl

- Exhaust gas recirculation

- Variable geometry turbocharger

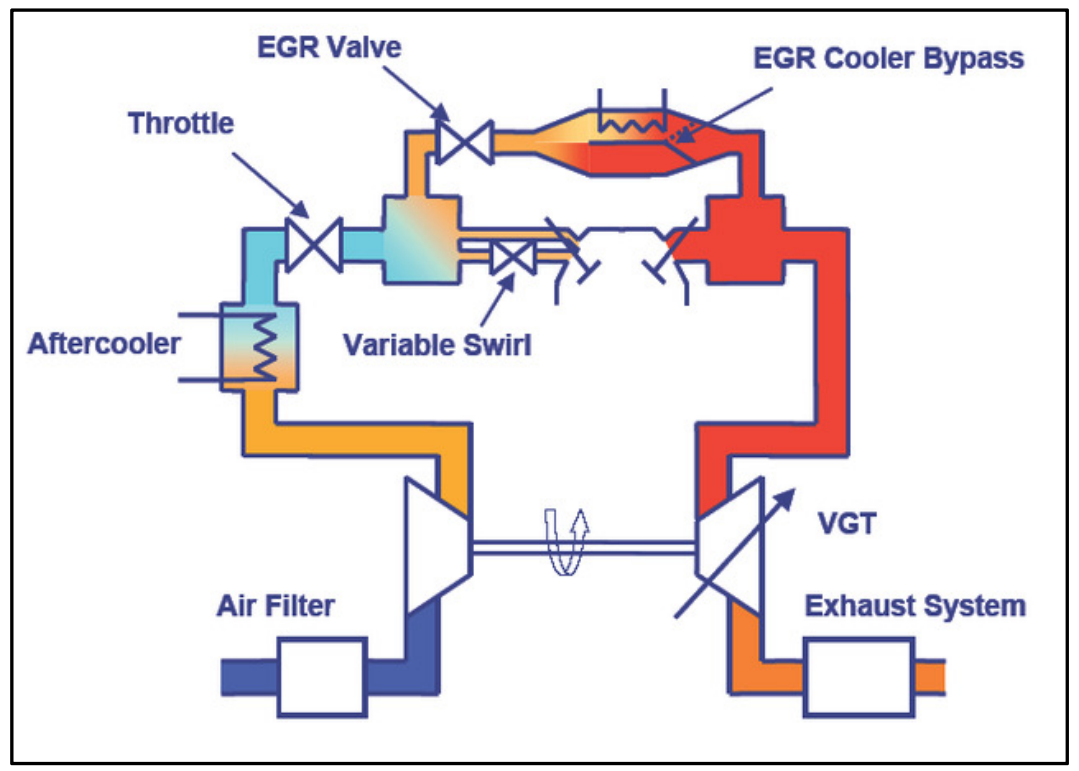

Figure 2.11 System diagram of auxiliary functions [17]

The WL-C engine implements Variable Swirl Control (VSC) and has two separate inlet ports, one of which can be opened and closed when required. At low engine speeds, the secondary port (known as the charge port) is closed, so the air is drawn into the cylinder through the first port only, producing a swirling motion in the cylinder creating a homogenous air/fuel mixture. At higher engine speeds (above 2300 RPM) however, the cylinder is unable to draw air in fast enough through a single port, so the charge port is opened, allowing air to enter the cylinder faster. This VSC system therefore leads to higher efficiencies at lower RPM by encouraging the formation of a homogenous air/fuel mixture through increased swirling action inside the combustion chamber.

Exhaust Gas Recirculation (EGR) is a technique used to reduce the amount of nitric oxide compounds $\left(\mathrm{NO}_{x}\right)$ produced by the engine [18]. It achieves this by diluting the intake air with a percentage of exhaust gas, reducing the peak temperatures inside the cylinder. As $\mathrm{NO}_{\mathrm{x}}$ compounds are produced at high temperatures, a reduction of combustion temperature 
prevents these from forming. In the WL-C engine, the exhaust gas is cooled by passing through a heat exchanger before being introduced into the intake manifold by means of a variable control valve to control the percentage of exhaust gas recirculated.

Variable geometry turbo chargers (VGTs) are used on modern engines to optimise the efficiency of the turbocharger at various speed ranges. A control arm changes the orientation of vanes inside the turbine housing, directing airflow onto the turbine blades at the most efficient angle, creating the best boost pressure for a given speed.

These auxiliary functions, while important for ensuring optimised engine performance, are not critical to the initial development of the dual fuel project. However, in the future it may be possible to alter these parameters slightly to fine-tune the engine while running on dual fuel.

\subsection{Dual Fuel Engines}

Dual fuel engines are engines that run on two fuels simultaneously. They differ from alternative fuel engines in that they can not run on the alternative fuel alone; they need both to operate. This project focuses on dual fuel engines running on diesel and compressed natural gas $(\mathrm{CNG})$.

\subsubsection{Dual Fuel Technology}

The idea of adding a gaseous fuel to an existing diesel engine has existed since the 1920s when hydrogen was used experimentally as a secondary fuel source [19]. Most modern dual fuel engines use gaseous petroleum based fuels such as compressed Natural Gas (CNG), Liquefied Natural Gas (LNG) and Liquefied Petroleum Gas (LPG). CNG is the most popular choice due to its common availability and efficient burning characteristics [20]. One of the main drawbacks of dual fuel operation is that power output and efficiency suffer when the engine is running under low load conditions [21]. Therefore, dual fuel technology performs best when applied to engines which are operated under an almost constant load, such as large diesel generators, or highway trucks which spend most of their time driving on the open road with little variation in load.

The dual fuel method used in this project is known as Homogeneous Charge Compression Ignition (HCCI), as it has characteristics of both traditional gas and diesel engines. That is, 
the charge of air and fuel admitted to the cylinder is a homogeneous mixture and ignited through the heat of compression.

The dual fuel technology investigated and developed in this project uses compressed natural gas as a primary fuel, ignited by a small pilot injection of liquid diesel. While the underlying principles of dual fuel operation are well known, many details surrounding the specific technologies used commercially are proprietary and are not readily available. Older dual fuel systems rely on mechanical manipulation of engine components, but with modern common rail diesel engines it is possible to perform an electronic intervention and manipulate the sensory information received by the OEM ECU. In some cases, an external ECU is used to either supplement or replace the OEM ECU [1].

Despite dual fuel technology having been around for several decades, there are few commercially available dual fuel engines. Several third party companies produce dual fuel conversion kits which modify existing diesel engines in order to operate under dual fuel conditions. While there are several different approaches to running dual fuel, the outcome is the same; reduce the amount of diesel required to that of a pilot injection and introduce a gaseous fuel to the air mixture.

Because of differences in the combustion processes between gas and diesel, there will be losses in the thermal efficiency of a dual fuel engine [19]. Efficiency losses are a product of several contributing factors in the combustion process such as flame propagation and reduced compression ratio [22]. Diesel engines typically run lean (i.e. more air than required) as a way to mitigate unburned hydrocarbons and the production of soot. However, dual fuel can be run closer to a stoichiometric air/fuel ratio and produces a cleaner burn [23]. Feedback can be obtained through analysis of the exhaust gases to determine oxygen and hydrocarbon content as well as nitric oxides, and the engine parameters changed accordingly.

An example of an existing dual fuel conversion method installed on mechanically-injected fuel systems reduces diesel flow by limiting the travel of the control rack, the position of which normally determines fuel metering. Because the position of the control rack is proportional to the quantity of diesel injected, an actuator can be applied to electronically control pilot fuel. This is the method employed by DieselGas International Ltd on their commercial dual fuel conversion kits, shown in figure 2.12. 


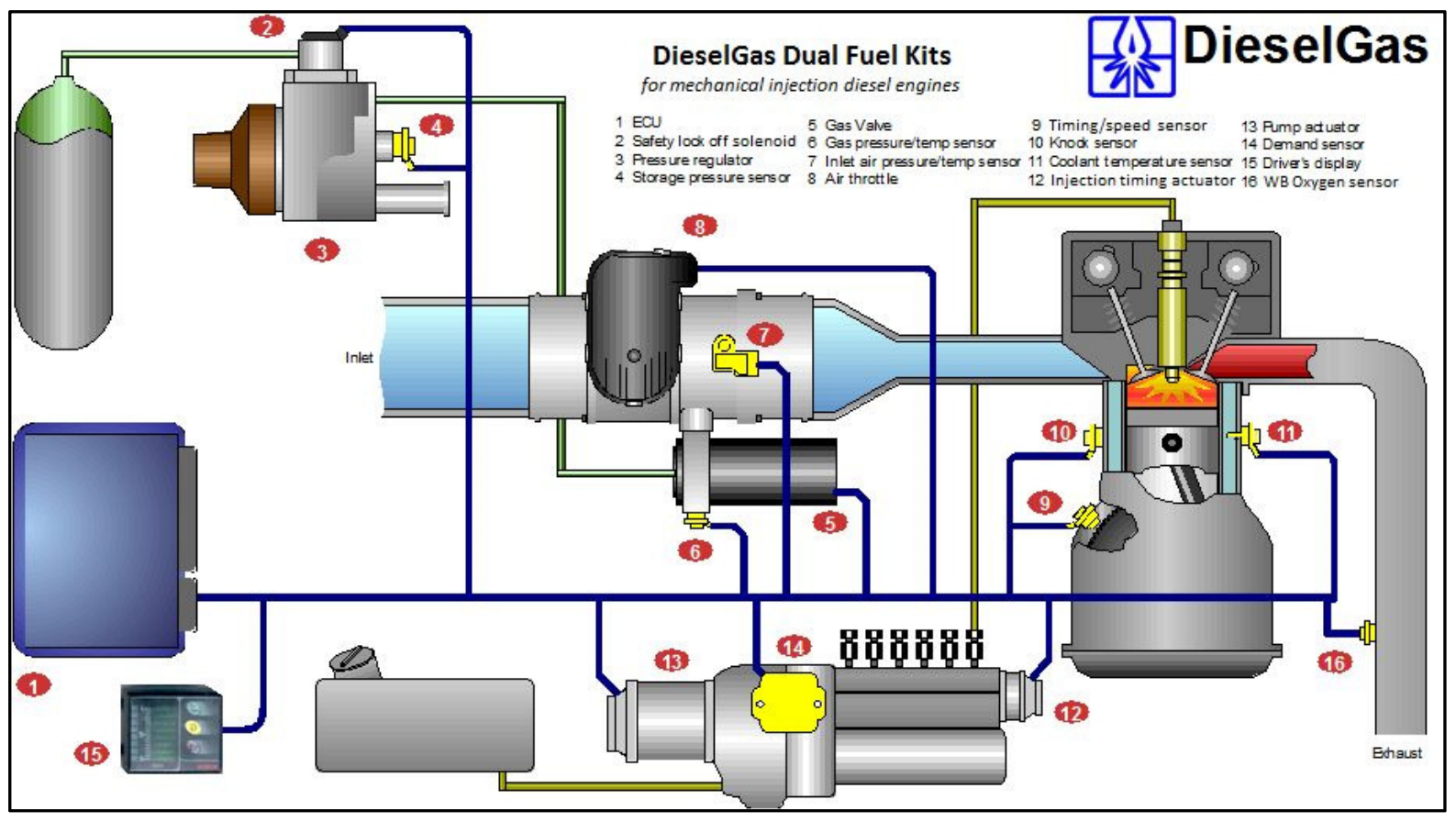

Figure 2.12 Diagram of current DieselGas dual fuel system (reproduced with permission, DieselGas International)

One of the biggest advantages to dual fuel technology is the ability to convert an existing diesel engine with minimal modification, while producing the same output performance characteristics as if it were running on $100 \%$ diesel. By ensuring the engine performance remains unchanged (or at least does not exceed manufacturer specifications), a dual fuel conversion kit will generally not void the OEM warranty [24].

Since no major modifications are made to the architecture of the mechanical components or structure of the engine (and all additions are mounted externally and can be disabled if necessary) the engine will still be able to run purely on diesel with the dual fuel system disengaged. This mitigates the danger of running out of gaseous fuel, and allows the operator of the engine to simply switch back to $100 \%$ diesel until more gaseous fuel can be obtained.

\subsubsection{DieselGas International}

DieselGas International is a local specialist alternative fuels engineering company with a 30 year history of designing and implementing proprietary natural gas dual fuel conversion systems for diesel engines. DieselGas has worked in several countries with several equipment manufacturers including Caterpillar and Robert Bosch GmbH.

The DieselGas product is a tailored dual fuel engine management system custom-fitted to production diesel engines, optimised to match the power output of the original engine 
specifications. The heart of the DieselGas product is the specialised ECU which monitors engine performance and modifies various engine parameters dynamically during operation. The DieselGas system is designed to meet EPS and Euro emissions requirements and can achieve a peak diesel substitution rate of up to $90 \%$.

The DieselGas system manages to attain these high substitution rates through intense mapping of the engine performance and optimisation of the fuelling ratios under various environmental conditions with respect to air temperatures and pressures. This produces a comprehensive fuelling table for each specific engine on which it is installed, providing dynamic control of fuel, air, and timing across all operating conditions and loads. This makes the DieselGas dual fuel conversion kits one of the most versatile, efficient, and cost-effective dual fuel systems on the market today.

The current generation of DieselGas dual fuel systems are designed for mechanical fuel injection systems, such as those driven by a mechanical jerk pump. In order to implement their dual fuel system on modern common rail engines, the technology must be developed further to interface with new electronic injection methods. This project aims to develop a new generic ECU for the DieselGas system allowing it to interface with common rail fuel systems on various modern diesel engines. 


\section{Concepts and First Prototype}

\subsection{System Concepts and Constraints}

\subsubsection{Overview}

Once the CRDI system was thoroughly analysed and understood, it was necessary to decide how the dual fuel system would fit within the existing hardware. Because this system is designed to supplement (not replace) the existing ECU, it is important that the dual fuel system be invisible to the OEM ECU, so as to disrupt normal operation as little as possible. The dual fuel system is designed to sit between the OEM ECU and the engine and reduce the amount of diesel being injected whilst also injecting gas into the inlet manifold. The basic overview of this system is shown in figure 3.1 below:

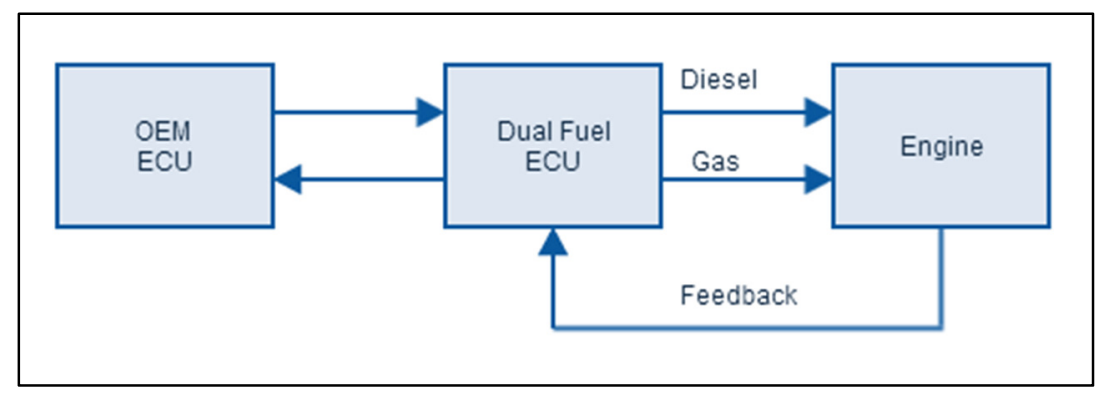

Figure 3.1 Block diagram of dual fuel ECU integration

The first major design decision was to base the new dual fuel ECU design on the existing dual fuel hardware developed by DieselGas International (DGI). This hardware was designed to work with mechanical injection systems, but the way it operates is largely similar to how the CRDI version will work. Instead of changing the position of the rack in the injector pump, the system will change the duration of injection pulses. It was therefore decided to use a 2-part system, where an existing DieselGas Controller will be used to control dual fuel operation, and a separate board would control the injection of diesel. This second board was coined the Diesel Injection Module, or DIM.

By using an existing DieselGas Controller as the starting point, most of the outputs and control systems are already implemented. The DIM will effectively replace the stepper motor which would normally control the rack, and also perform signal conditioning and timing calculations based on the outputs of the cam and flywheel sensors. The modified DieselGas 
Controller would determine how much of each fuel is required and communicate with the DIM to control diesel delivery (figure 3.2).

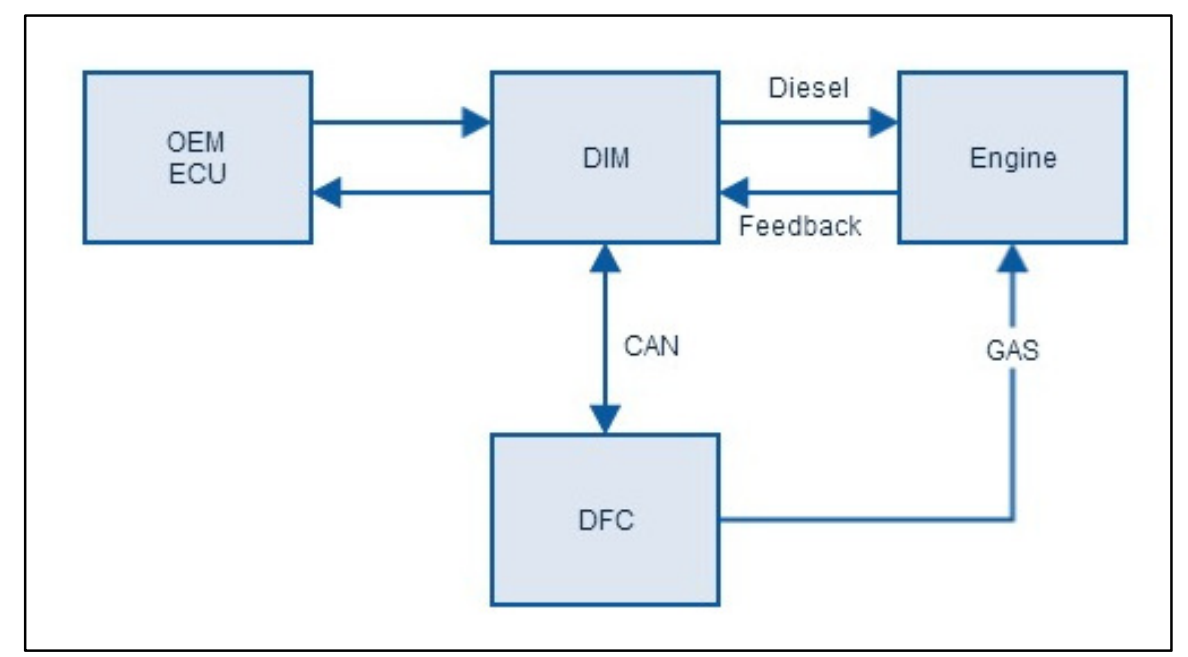

Figure 3.2 DIM and DFC implementation diagram

The new dual fuel system will operate in basically the same way as the old mechanicallyactuated system. During operation, the driver of the vehicle can select between two modes; diesel mode and dual fuel mode. In diesel mode, the engine runs as it would normally on diesel fuel (i.e. no intervention is required and no gas is injected). In dual fuel mode, the dual fuel system will operate and begin diesel substitution.

Because of the new two-part system for the original prototype, a new nomenclature was introduced to distinguish the controllers from previous versions. The modified DieselGas Controller (DGC) now becomes a Dual Fuel Controller (DFC), which controls the handling of dual fuel operation, and tells the Diesel Injection Module (DIM) how much diesel fuel to deliver to the engine.

The DIM is also designed to handle the pass-through and analysis of the timing signals from the engine, so the DIM can know what position and phase the engine is in, allowing for correct timing of diesel injection. The primary inputs to the DIM are the injector wires from the OEM ECU to the engine, and the timing signals from the engine to the OEM ECU. The main outputs are the injector pulses from the DIM to the engine and the timing signals to the OEM ECU. The DIM is in communication with the DFC, from which it receives information about how much diesel to inject and when. 


\subsubsection{Concepts}

\subsubsection{Switching systems}

In order to reduce the diesel fuel delivery to the engine, the pulses of current to the diesel injectors must be modified. However, any modification to these signals must remain invisible to the OEM ECU, that is, the OEM ECU must be 'tricked' into thinking it is still driving the injectors normally. The chosen method of performing this task is to have a set of dummy loads which will be connected to the OEM ECU whenever the DIM is in control of fuelling. When the engine is running in pass-through mode, the injectors are switched back in and are controlled solely by the OEM ECU.

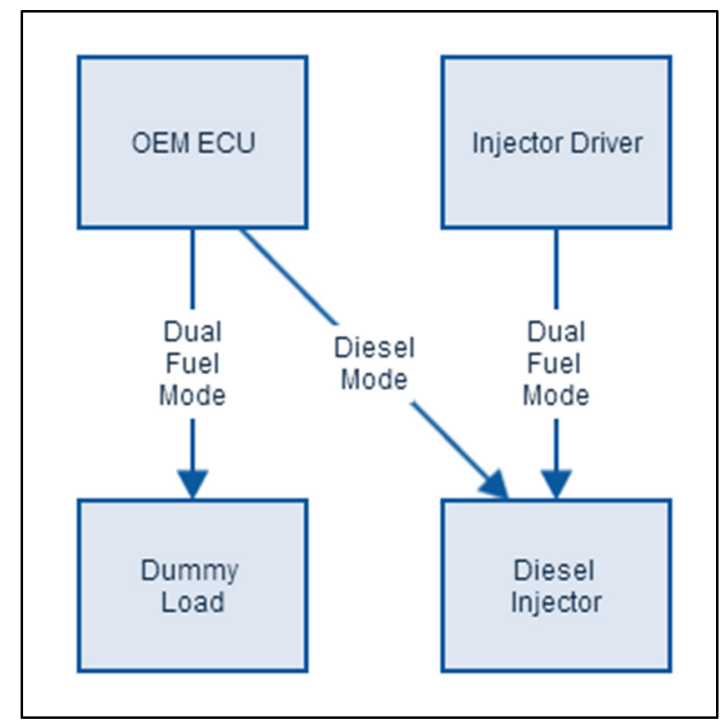

Figure 3.3 Modes of operation

The diagram in figure 3.3 shows the basic operation of the switching system. When in diesel mode the injectors are connected to the OEM ECU as they normally would be without intervention. In dual fuel mode, the injectors connected to the OEM ECU are replaced by dummies, and the DIM can take over diesel injection.

\subsubsection{Injector Driving}

The first method of modifying diesel fuel delivery investigated is to drive the fuel injectors independently of the OEM ECU. The DIM, whilst monitoring the timing signals of the engine, will determine where in the cycle to begin fuel delivery and how much fuel is to be injected, sending current to the injector and holding it open for the appropriate length of time. 
This method is considered to be the most straightforward way to take over the fuelling as it allows full control of diesel delivery. This provides flexibility in the design to make minute changes $(\sim 1 \mu \mathrm{s})$ to the diesel injection time, and also to change the timing of fuel delivery independent of the OEM ECU.

\subsubsection{Injector Gating}

Another potential method of reducing the diesel fuel delivery is to use the existing injector signals from the OEM ECU, and simply cut the current pulses short. This has the effect of prematurely closing the fuel injector, thus reducing the amount of fuel delivered to the cylinder. This method of gating the injector pulses has the added advantage of using the existing signals produced by the OEM ECU to drive the injectors, eliminating the requirement for injector drivers and the associated complex circuitry. However, because the OEM ECU injects diesel according to its own fuelling algorithm, it will be necessary to phase-shift the timing signals from the engine in order to change the timing of the injection pulses relative to Top Dead Centre (TDC), the top of the compression stroke.

Changing the timing of the engine signals is not difficult when retarding (delaying) the original signals, but becomes more complex when advancing the signals, as the system must effectively predict the future and make assumptions about how the signals are going to behave. This requires more complex code and processing from the processor.

\subsection{Hardware development}

\subsubsection{Injector Switching}

For both concepts mentioned previously, the one common element is the switching system. This allows the DIM to override the injection signals coming from the OEM ECU and mask the intervention by connecting dummy loads to the OEM ECU in place of the actual injectors.

In the most basic sense, the switching componentry consists of a number of switching devices which directs the injector current through either the diesel injector or a dummy load of similar impedance. This allows the injectors to be driven by either the OEM ECU in passthrough mode or the DIM in dual fuel mode. When in dual fuel mode, the OEM ECU is connected to the dummies, which register as real injectors, masking the fact that the diesel injection pattern is being manipulated. 
As the switching system is fundamental to the viability of this project, it was the first subsystem to be built and tested. This proof of concept tests the functionality of the switching components selected, the dummy loads, and the OEM ECU response. The initial design for this proof of concept is to switch a single dummy load in place of a single injector. This test is performed on the BT50 engine and the ECU response monitored via an OBD scan tool connected to a laptop to record any error codes.

\subsubsection{Schematic and component selection}

To conceptualise the switching hardware, a general overview was imagined using ideal switches. The basic principle is shown in figure 3.4 below:

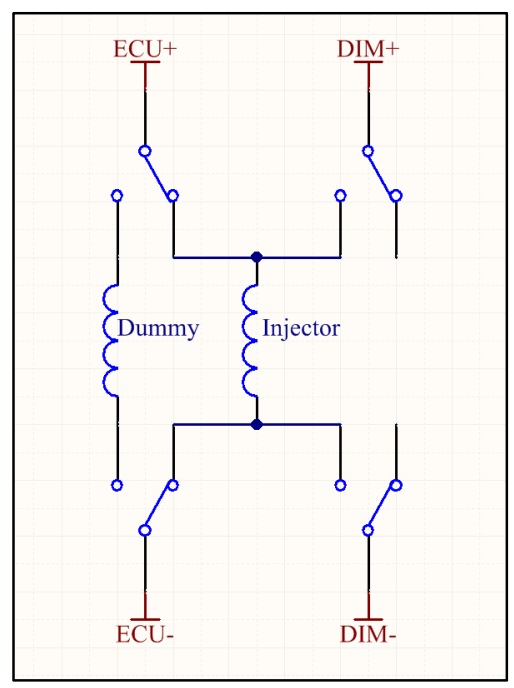

Figure 3.4 Switching hardware general overview

Figure 3.4 shows how the injector current from the OEM ECU is steered through either the injector or the dummy load (all switches operated simultaneously), and how the DIM is then switched in to take over diesel injection. This configuration forms the foundation of all subsequent switching designs and is a fundamental subsystem of the DIM.

Two different switching elements were investigated; electromechanical relays and field effect transistors (FETs). Relays were considered because of their ability to handle high currents for sustained periods (and so are electrically hardy), and because they can be electrically isolated from the control circuitry, which is useful because the injector lines are floating. They also allow us to have multiple pole switches on a single throw. However, they are quite large devices and because of their size, would not be suitable for the task as the number required would take up too much space on the Printed Circuit Board (PCB). Because relays are fundamentally mechanical devices, it is likely that they would also become failure points 
through mechanical wear and stresses brought about through vibration and heat from being near an engine.

FETs can come in much smaller packages than relays, and although they can not handle the high sustained currents relays can, an N-channel MOSFET can pass the current required for this application $(\sim 15 \mathrm{~A})$, so long as it is not for too long (i.e. $<1 \mathrm{~s}$ pulses). The other added advantage FETs have is that they are solid state and thus do not contain any fragile moving internal parts. The main drawback to using FETs is the requirement for external control circuitry such as gate drivers and isolated power supplies to switch the floating injector lines. This control hardware needs to be replicated for each set of switches, but still takes up considerably less space than a set of relays.

Since FETs are a single switching element, the schematic was modified to account for this. Now that the dummy load is only going to be driven by the OEM ECU it does not need a switching element on both the high and low sides, so only three transistors are required to perform the switching. A diagram demonstrating this arrangement with single ideal switches is shown in figure 3.5 :

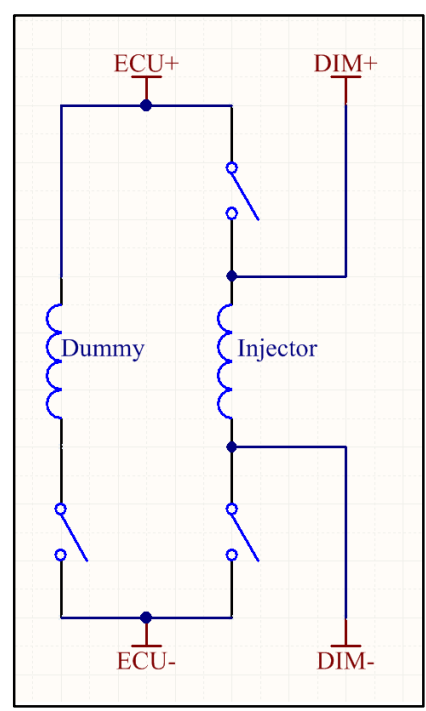

Figure 3.5 Simplified switching hardware

The FETs chosen for the initial proof of concept were 3N10L N-channel MOSFETs from Fairchild Semiconductor [25]. They can handle 33 A continuous at $100 \mathrm{~V}_{\mathrm{DS}}$ and have an on-resistance of $52 \mathrm{~m} \Omega$. These FETs are more than capable of handling the voltages and currents from the ECU (typically up to $80 \mathrm{~V}$ and $10 \mathrm{~A}$ ). To drive the FETs, there are two requirements: an isolated power supply to provide the requisite gate voltage, and a gate driver to turn the FET on and off as required. The power supplies must be isolating because the high 
and low sides of the injectors are floating. Thus, to provide an adequate gate-source voltage to turn the FETs on, the power supplies must provide a positive voltage relative to the source pin of each FET. The power supplies chosen were VBT1, $12 \mathrm{~V}$ isolated output DC/DC converters, from CUI [26]. These take an input of $12 \mathrm{~V}$ and produce an isolated $12 \mathrm{~V}$ output.

To drive the gates of the FETs an opto-coupled gate driver is used to isolate the control circuitry from the switching circuitry. The selected drivers are Vishay VO3120 IGBT and MOSFET drivers [27], which can deliver 2.5 A of current to the gate, resulting in a very fast turn-on time ( $\max 16 \mathrm{~ns}$ with gate charge of $40 \mathrm{nC}$ ). The gate drivers are controlled with a simple switch (S1, figure 3.6) which selects between routing the injector current from the OEM ECU through either the injector or through a dummy load. This switch is manually operated for this proof of concept to minimise complexity and focus on the performance of the switching elements. The final schematic can be seen in figure 3.6 below:

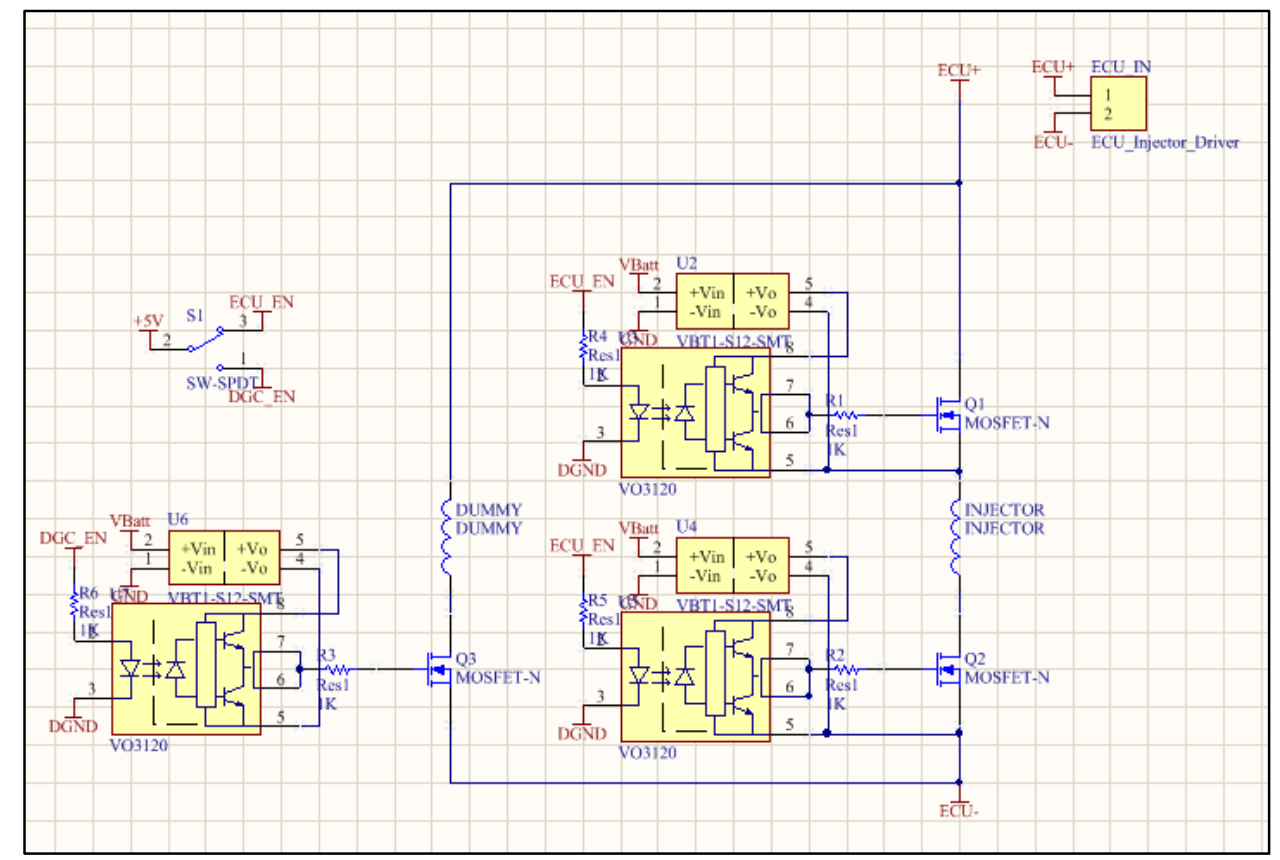

Figure 3.6 Prototype switching hardware schematic

\subsubsection{Dummy Loads}

To trick the ECU into thinking it is still driving an injector, a dummy load of similar electrical characteristics is connected to the ECU output. To determine what impedance the dummy load should have, a number of injectors were measured in-situ with a multimeter. The Bosch injectors in the BT-50 showed a DC resistance of $3.3 \Omega$. Initially, a purely resistive dummy load was tested by plugging a $3 \Omega$ resistor into the injector connector in the BT-50. 
However, this immediately caused an error code as the ECU could recognise that the impedance of the dummy load was not the same as a solenoid injector with a high inductance.

Next, the inductance of the injector was measured. Since the injector is a solenoid, and thus a large inductor, it made sense that the inductive component would have an impact on how the ECU would recognise it. The inductance of the injectors was measured and found to be approximately $150 \mu \mathrm{H}$ at $10 \mathrm{kHz}$. This reading provided sufficient information to try making a dummy load by hand. Since the solenoid is basically a coil of wire around an iron core, a suitable facsimile was created by wrapping enamelled wire around a steel bolt until the inductance and resistance were similar to that of the injector.

\subsubsection{Testing}

The PCB was produced on a LPKF PCB mill out of single sided copper board and tested on the WL-C engine in the BT-50. With the dummy load switched in prior to cranking, the engine would not start. However, with the engine already running, the dummy could be switched in line with the OEM ECU without immediately causing the engine light to illuminate as it would when an injector was unplugged. While the engine would run rough (as it is no longer firing on one cylinder) the OEM ECU would not immediately recognise the open-circuit fault as before, thus indicating that the OEM ECU did indeed recognise the dummy load as an actual injector. Figure 3.7 shows the proof of concept plugged into the engine and a prototype dummy load.

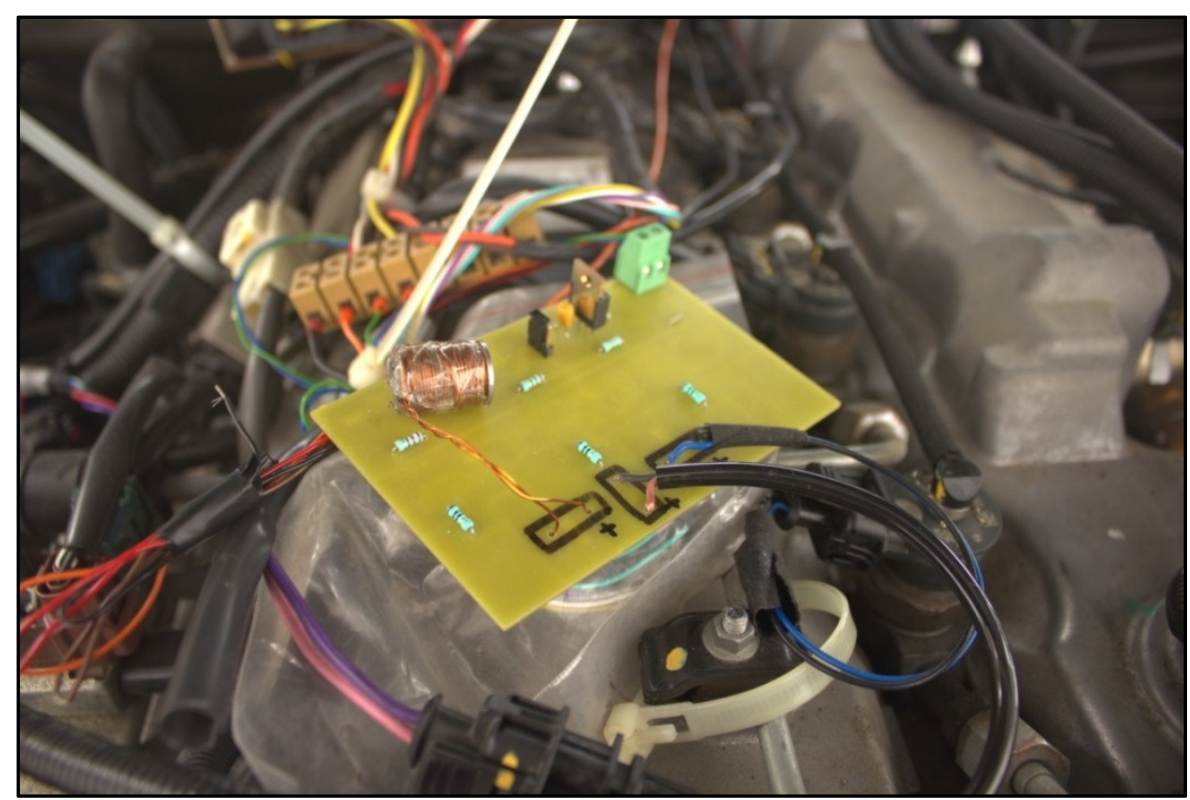

Figure 3.7 Prototype switching hardware plugged into BT-50 engine 
However, after about 30 seconds of running, the engine light came on for a different reason; this time it recognised a misfire on cylinder one. This indicates that the ECU monitors flywheel acceleration, and can infer from this data when a cylinder is misfiring, and also most likely an imbalance in cylinder power. This means it can likely change the fuelling ratios for each cylinder to ensure smooth running, which may become important later in the design cycle when running on dual fuel.

\subsubsection{Proof of Concept}

This testing showed that the switching method of using FETs is a reliable and effective method of switching between OEM and dual fuel modes of operation. When running in diesel mode, the ECU can drive the injectors as per normal via the FETs, and when running in dual fuel mode, the injector current from the OEM ECU can be routed through the dummy loads without seeing any error in the circuit, and thus not producing any error codes. As it is such an integral part of the DIM, proving that the switching method and appropriate selection of dummy load can effectively hide the intervention of the DIM from the OEM ECU, means that development of the subsequent subsystems can continue.

\subsubsection{Injector Driving}

\subsubsection{Overview}

The first DIM prototype uses an injector driving circuit to control the diesel injectors by itself. This concept is chosen for initial development as it allows the most direct control of the injectors, and therefore fuel delivery. Having total control over diesel flow will make fine-tuning of the engine performance possible and should allow for better performance on dual fuel. Before an entire prototype was designed, the relevant subsystems were tested in a proof of concept designed to drive a single injector and deliver fuel into one cylinder. The block diagram in figure 3.8 shows how the proof of concept for the injector driving DIM operates.

The main features of this concept are:

- Injector Driver

- Switching components

- Current monitor

- Microcontroller 


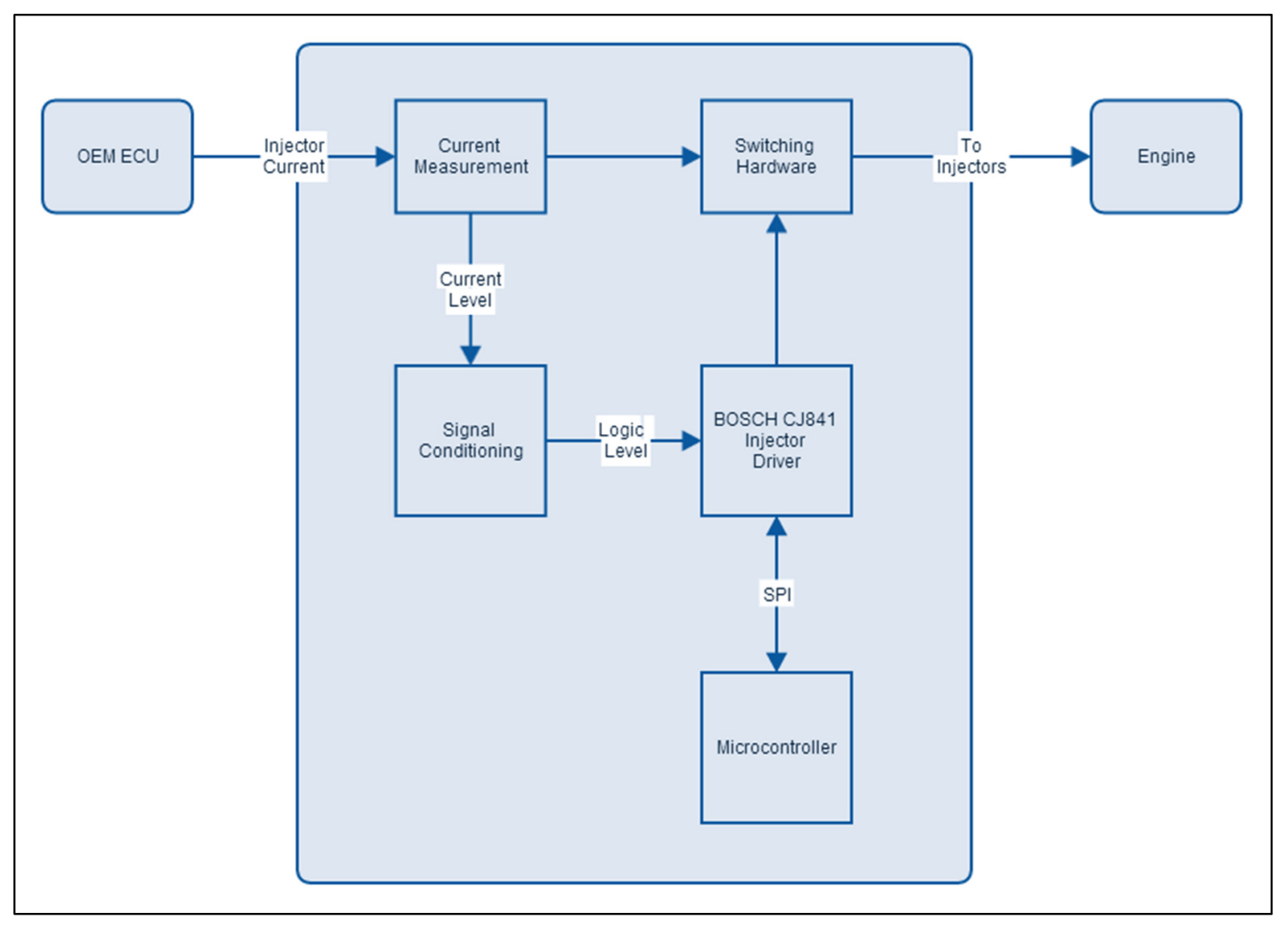

Figure 3.8 DIM overview

The basic mode of operation for this proof of concept is to mimic the injector signals of the OEM ECU sent to a single cylinder by replicating them with independent injector driving hardware.

In diesel mode, the injector current from the OEM ECU is passed straight to the injectors. However, in dual fuel mode, the OEM injector current is sent to the dummy loads, and a Bosch injector driver chip is used to drive the injectors. For this proof of concept, the injector driver chip is triggered by a current sensing circuit which produces a logic level signal when it detects current from the OEM ECU. In this way, the injector driver chip simply mirrors the injection pattern of the OEM ECU, proving the concept of being able to drive the injectors with this setup without having to design fuelling tables beforehand. A microcontroller is added to configure the various parameters of the injector driver chip.

\subsubsection{Schematic}

The circuit is divided into 4 main areas: the switching hardware, the injector driver, microcontroller, and power supply. 


\subsection{Injector Driver}

The first DIM prototype is based around the Bosch CJ841 injector driver chip. This is a proprietary chip developed by Bosch specifically to drive automotive injectors. It has numerous features which make it an ideal platform to base the DIM around, such as the ability to control 4 injectors on 2 independent banks (injectors are paired on each bank), short circuit protection, integrated DC/DC boost converter control, and integrated diagnosis and configuration via a Serial Peripheral Interface (SPI).

The CJ841 has an internal state machine which controls the various stages of the injection current pattern. Up to 8 different stages can be utilised to give an injector current pattern that best suits the individual injectors and engines to be controlled. Each of these phases can be interrupted by various fault conditions or a signal from the external logic control to turn the injector off. For the purposes of this design implementation we will only use the two main phases of operation; the pickup phase and the holding phase, in order to reduce complexity of the proof of concept.

In its idle state, the CJ841 sends no current to the injector. When the logic input level is triggered (active LOW), signalling injection to start, three events take place:

- The high side switch for that bank of injectors goes HIGH, applying battery voltage to the high side of the injector;

- When the high side switch first turns on, the booster high-side switch also goes $\mathrm{HIGH}$, turning on the DC/DC BOOST converter and increasing the voltage on the high side up to $\sim 100 \mathrm{~V}$.

- The corresponding low side switch (which is injector specific) goes HIGH, pulling the low side down, allowing current to flow through the specified injector.

The high BOOST voltage is generated to increase the voltage across the injector solenoid as quickly as possible, allowing fuel delivery to begin much quicker than if the battery voltage was simply applied. This pick-up phase lasts just long enough to get the injector fully open, and then the holding phase begins. During the holding phase, the voltage across the injector solenoid is decreased to the battery voltage and pulsed to maintain a specified holding current. This is sufficient to hold the injector open, but does not draw as much current, allowing fuel to still flow but using less power. 
The lengths of these phases, current limits, and various diagnostic values can all be set and read by accessing the appropriate registers of the injector driver via SPI. The configurable registers and their basic functions are described below in table 3.1:

Table 3.1 Injector driver registers and functions

\begin{tabular}{|l|l|}
\hline Register Name & Function \\
\hline PARACON1 & Booster current, pick up current, holding current, current hysteresis \\
\hline PARACON2 & Booster phase timeout, pick up phase1 timeout, booster delay \\
\hline PARACON3 & Booster current control, pick up phase timeout, number of boost cycles \\
\hline PARACON4 & DC/DC Boost current control, CLK frequency \\
\hline PARACON5 & Low side switch enable, DC/DC boost enable, state machine control \\
\hline
\end{tabular}

The diagnosis registers (DIAGNOSIS0...2) provide information to the errors detected by the chip. The errors include:

- Over temperature

- Missing clock

- Under voltage

- Short circuits

- No load

- Booster timeout

To set the chip configuration and read the diagnosis registers, a microcontroller is added to the design to act as a controller and interface allowing computer control and observation of the performance of the driver. An Atmega 328 is used, running the Arduino bootloader. This allows a simple program to be written to configure the chip on start-up and report any errors via a serial connection.

To drive the clock of the CJ841, a $1 \mathrm{MHz}$ clock generator is used to provide a clean square wave signal required by the injector driver. The injector driver is configured to accept a 1 MHz clock signal by the microcontroller on start-up. The Atmega 328 microcontroller used is chosen because ready-made breakout boards are available, and can be surface-mounted on 
the PCB as a simple drop-in module. The microcontroller runs the Arduino bootloader and instruction set, making coding very simple and easily updated via an external serial connection.

The external circuitry required for the DC/DC boost converter is not well documented in the datasheet. No indications are given as to the values of the inductor and capacitor, nor the switching frequency at which the FET will be driven. An online calculator was used to make an estimation of the required component values, based on a maximum switching frequency of $1 \mathrm{MHz}$ (the clock frequency of the chip) which led to the selection of an inductor of $10 \mu \mathrm{H}$ and capacitor of $4.7 \mu \mathrm{F}$; the estimated values of which should be sufficient for initial testing. Standard footprints were chosen for these components which would allow interchangeability of these parts in case the values were off by a significant amount.

The CJ841 receives feedback from the output of the boost converter via a voltage divider, which can be varied to increase or decrease the BOOST voltage required. For this implementation, a potentiometer was added so the BOOST voltage could be modified easily during testing. The chip also monitors the current flowing through the FET (Q4, figure 3.9) by reading the voltage across a $1 \mathrm{~m} \Omega$ shunt resistor. This allows the chip to shut off the FET if the current gets too high, for example if the inductor becomes shorted. The BOOST circuit diagram is shown in figure 3.9 .

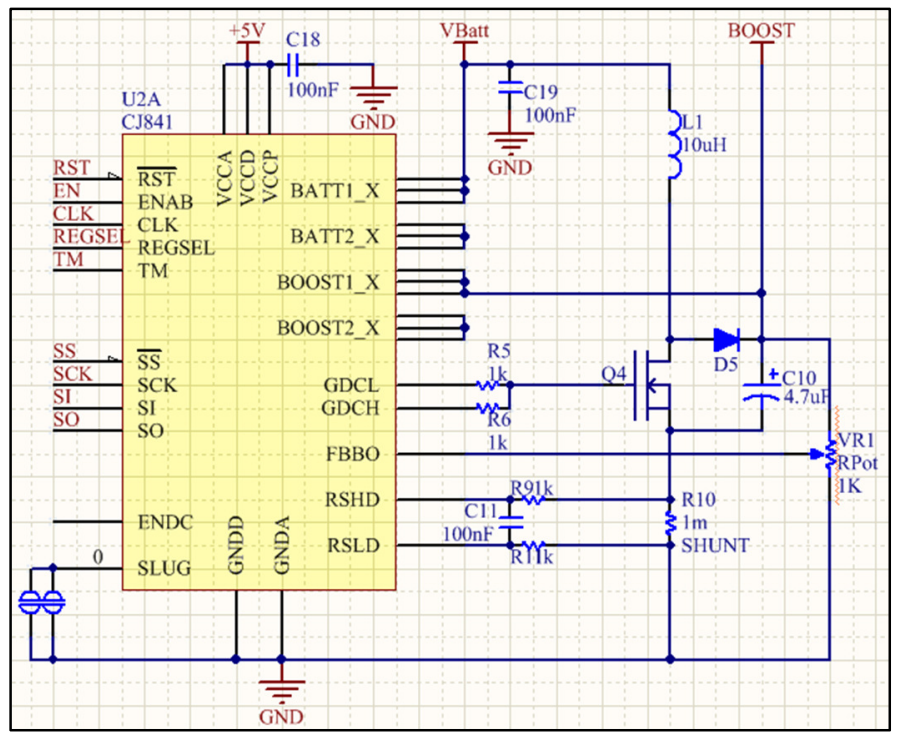

Figure 3.9 Bosch CJ841 injector driver connections 


\subsection{Switching Components and Current Monitor}

The switching components for this proof of concept are similar to the previous switching component test, but use different gate drivers and in a different configuration (figure 3.10). Since the two low-side FETs have a common source, a single power supply can be used to drive both. A single gate driver is used to drive the high side FET.

The gate drivers chosen were the IRS2117 single channel driver [28] and the ADuM7234 isolated precision driver [29]. The IRS2117 is an automotive-grade high-side driver which can handle drain-source voltages up to $600 \mathrm{~V}$. This is useful because of the presence of high voltage inductive spikes produced by the injectors and dummy loads. The ADuM7234 has two independent channels to drive gates and can be supplied by independent power supplies. This may be useful in reducing the number of gate drivers required on the PCB if one chip can drive both the high and low sides of the switching FETs. This component was chosen for this application to test its performance. Both gate drivers are driven by the VBT1 12V isolated power supply ICs.

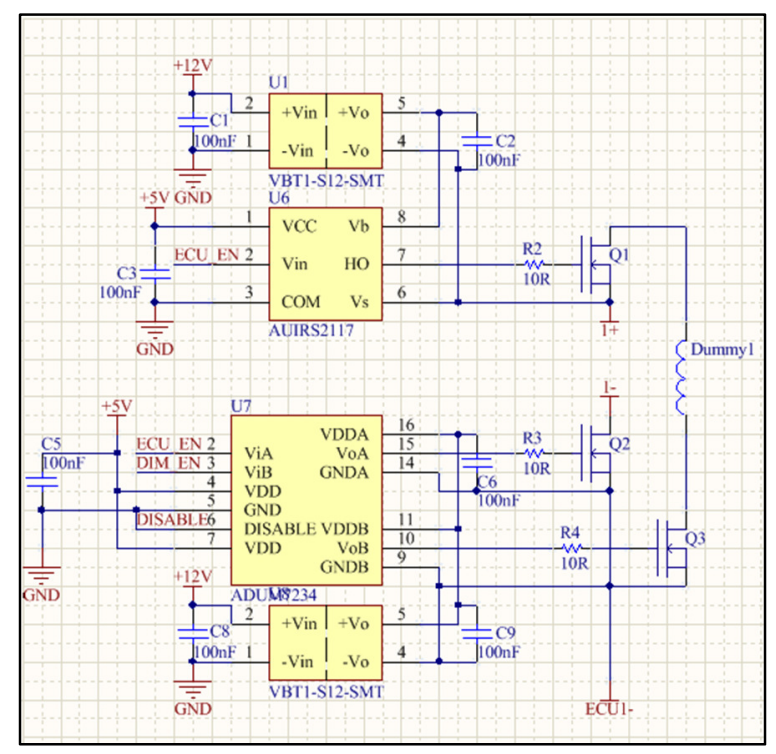

Figure 3.10 DIM proof of concept switching hardware

To monitor the injector current from the OEM ECU, a current transducer was placed in series with the injector output, before the high-side switch. The current transducer is an Allegro ACS711 Hall Effect linear current sensor [30] which can detect current in two directions and produce an output voltage proportional to the current flowing through it with a resolution of $55 \mathrm{mV} / \mathrm{A}$. This current transducer is used to drive one input of a comparator to produce a trigger for the injector driver. In this configuration, the injector driver will effectively be able 
to mimic the current pulses from the OEM ECU and drive the injector in the same pattern. The goal is to have the engine running like this in 'pass-through' mode, where the engine is running solely on diesel, but with the injection current coming from the on-board injector driver and not the OEM ECU. This will demonstrate the operation of the various subsystems that will then make up the initial DIM prototype.

The output of the current transducer at $0 \mathrm{~A}$ is $2.5 \mathrm{~V}$ and the voltage swings $2.5 \mathrm{~V}$ above and below this, proportional to the magnitude and direction of current flow (up to 12.5 or $25 \mathrm{~A}$ depending on the configuration). The current sensor is set up such that the injector current induces a positive voltage swing from the neutral position. This output is fed into a comparator, as shown in figure 3.11 below. The output of the comparator is a digital signal $(0-5 \mathrm{~V})$ which triggers the input channel of the injector driver. The full schematic for this proof of concept is shown in figure 3.12.

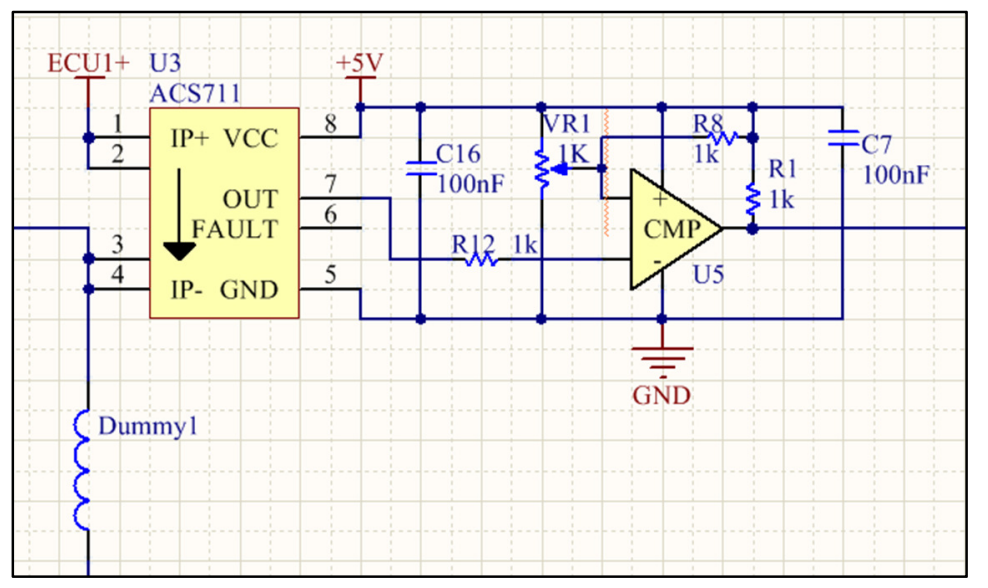

Figure 3.11 DIM proof of concept current sensing and signal conditioning

It is important to consider the propagation delays these systems would cause to the injection pattern so each of the individual sub systems are analysed to calculate a total propagation delay. The first stage to consider is the rise time of the output current from the BT-50. The current rises from 0 to $19.2 \mathrm{~A}$ in approx. $80 \mu \mathrm{s}$. The current transducer on its most sensitive setting has a sensitivity of $110 \mathrm{mV} / \mathrm{A}$. This means that for an output of $0.5 \mathrm{~V}$ above the zero point, there must be $4.5 \mathrm{~A}$ of current flowing. The OEM ECU current signal takes approx. $4.2 \mu \mathrm{s}$ to increase by $1 \mathrm{~A}$, so will take $18.75 \mu$ s to rise to the level at which the current sensor will output $0.5 \mathrm{~V}$ above the zero point. As this rise time is far slower than the rise time of the current sensor $(3.5 \mu \mathrm{s})$, the only additional delay is the propagation delay of the sensor which is $1.2 \mu \mathrm{s}$. The next delays come in from the comparator and the injector driver. 
The LM397 comparator chosen has a propagation delay of $0.44 \mu$ s and the injector driver has a nominal delay of $3 \mu$ s from receiving the signal to outputting injector current. This means that the total propagation delay from the time the OEM ECU begins producing current to the time the injectors receive current from the CJ841, is approx. $4.64 \mu \mathrm{s}$. This delay can be considered constant no matter what the speed of the engine, so at higher speeds, the engine will have rotated more in that time than at idle. Therefore, this propagation delay must be calculated in terms of angle of rotation at the highest and lowest speeds to determine the best and worst case scenarios.

At idle (approx. 400 RPM), a delay of $4.64 \mu$ s corresponds to an angle of 0.011 degrees. This means that the fuel delivery is retarded by 0.011 degrees. However at full speed (4000 RPM), this angle becomes 0.11 degrees and while ten times more significant, should still not adversely affect the performance of the engine.

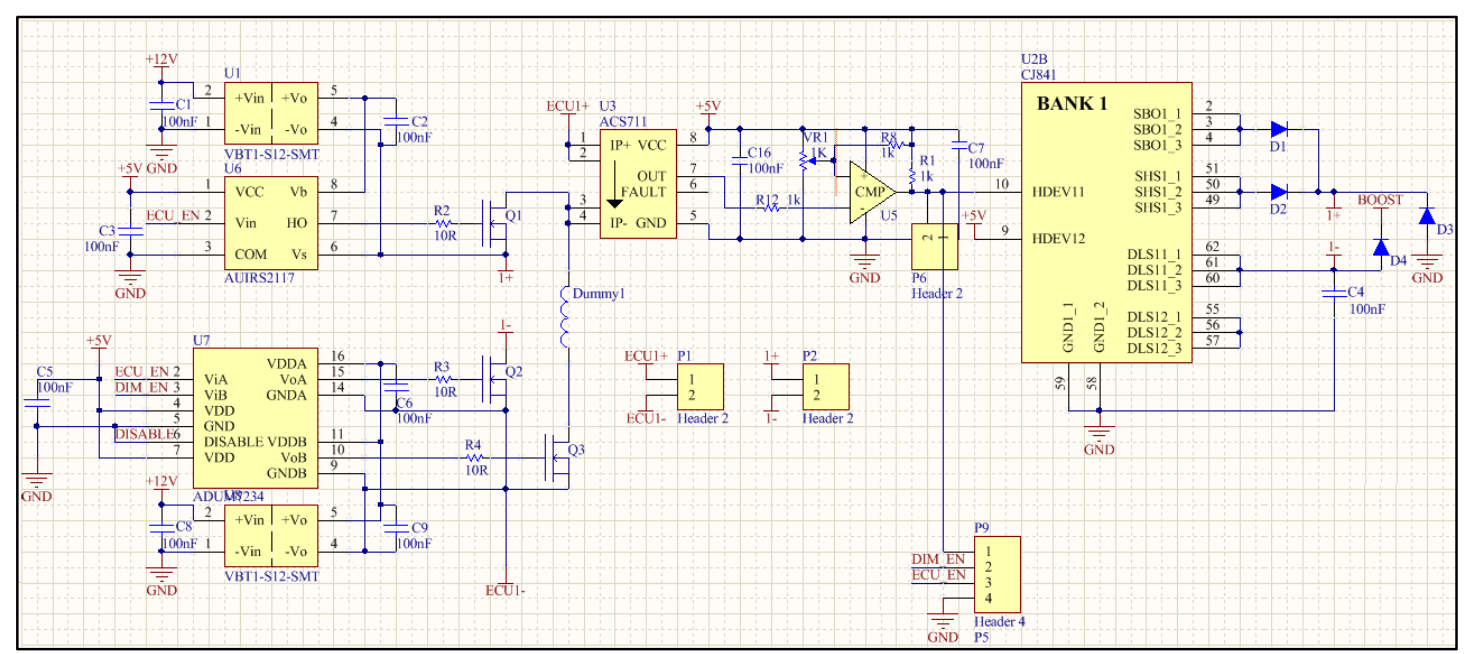

Figure 3.12 DIM proof of concept schematic

\subsubsection{PCB}

The PCB layout is critical to the good operation of the system, and several design considerations are taken into account. The main one is the high current path for the injector signals. Not only are there the high current signals from the OEM ECU, but also the output of the CJ841. The PCB tracks for these signals are kept as short and wide as possible to provide a low resistance path for the high current pulses. Where these signals have to travel through the PCB, many vias are used to carry the current through the board. The layout for this PCB can be seen in figure 3.13, where the ground planes on either side have been omitted for clarity. 


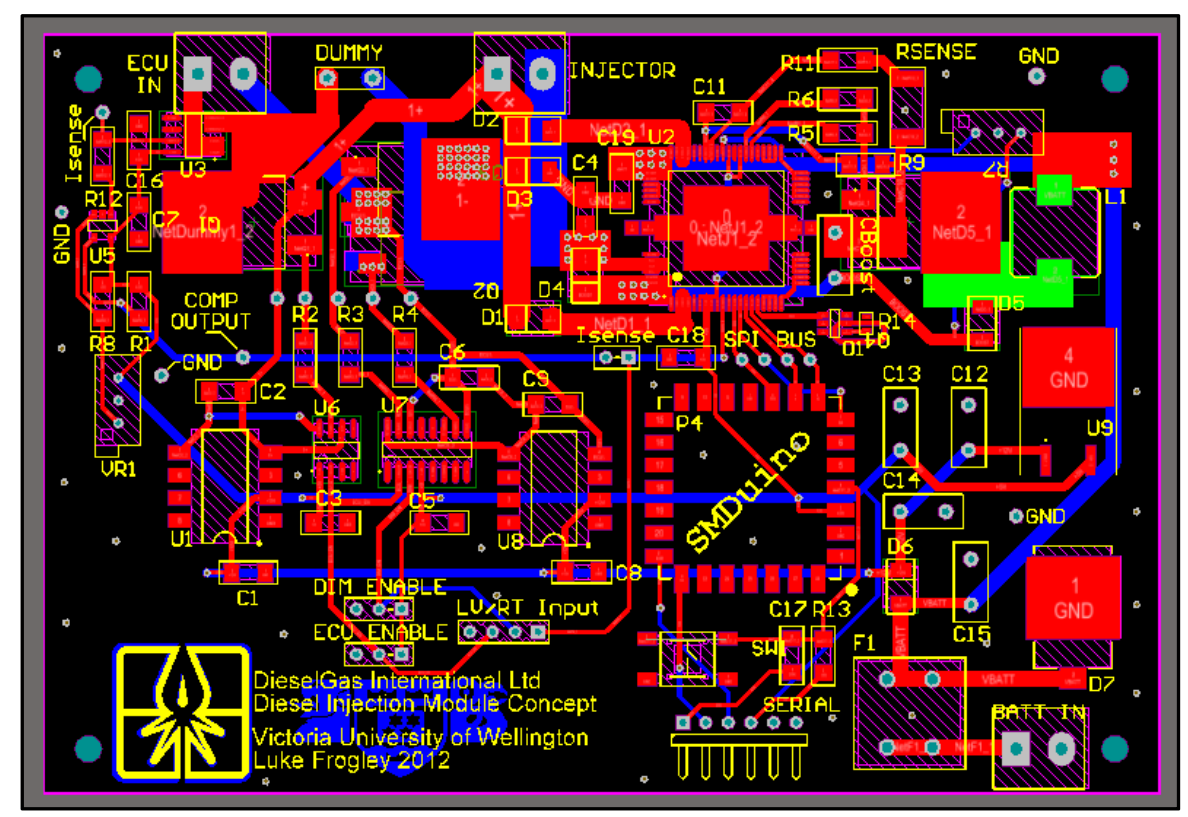

Figure 3.13 First DIM proof of concept PCB layout

When designing hardware for automotive applications, it is important to consider the conditions in which they will operate. Some of the biggest dangers to circuitry in an automotive application are temperature, vibration, and moisture. As an engine heats up, it can produce very high temperatures, which can affect the operation of components nearby. For this reason, automotive-grade components are often stress-tested to ensure their full operation across a wide range of temperatures. This not only includes extreme heat, but also extreme cold, as vehicles can also experience very low temperatures when used in such climates. For this reason, automotive grade components are used where available.

Vibration and shock damage to components is another danger to automotive hardware. High and low frequency vibrations from engine and road use can stress components and mountings, and can lead to fracturing, bending or breaking of delicate components such as silicon chips etc. Where appropriate, surface mount devices are used, and components with a low centre of gravity were chosen for through-hole components. This is not as important for a prototype such as this, but it is still important to think ahead to production when choosing particular components for a specific application.

For example, the FETs used could have been through-hole, but since a surface mount (SMD) version was available, they were selected as they would likely be similar to those used in the final version. The same principles were applied to the selection of capacitors; no leaded capacitors are used, as their high centre of gravity (like that of a standard electrolytic cap) will eventually succumb to the vibrations and fatigue or break. 
The PCB is mounted on a piece of acrylic which serves as insulation from the underside as it will be sitting in an engine bay.

\subsubsection{Testing}

The PCB is populated and tested in stages. First the power supply, then the switching hardware, and finally the injector driver chip and boost converter components. Unfortunately, there was an error in the footprint size for the microcontroller breakout board, which turned out to be too small. This was remedied by lifting the breakout board up and mounting it on legs which made contact with the erroneous footprint.

There were a few errors related to layout and component footprints which were relatively easily solved, such as the SPI connection between the microcontroller and injector driver chip. The data lines MOSI and MISO were transposed and required a couple of jumper wires to connect them correctly. A picture of the board during population and testing can be seen in figure 3.14 .

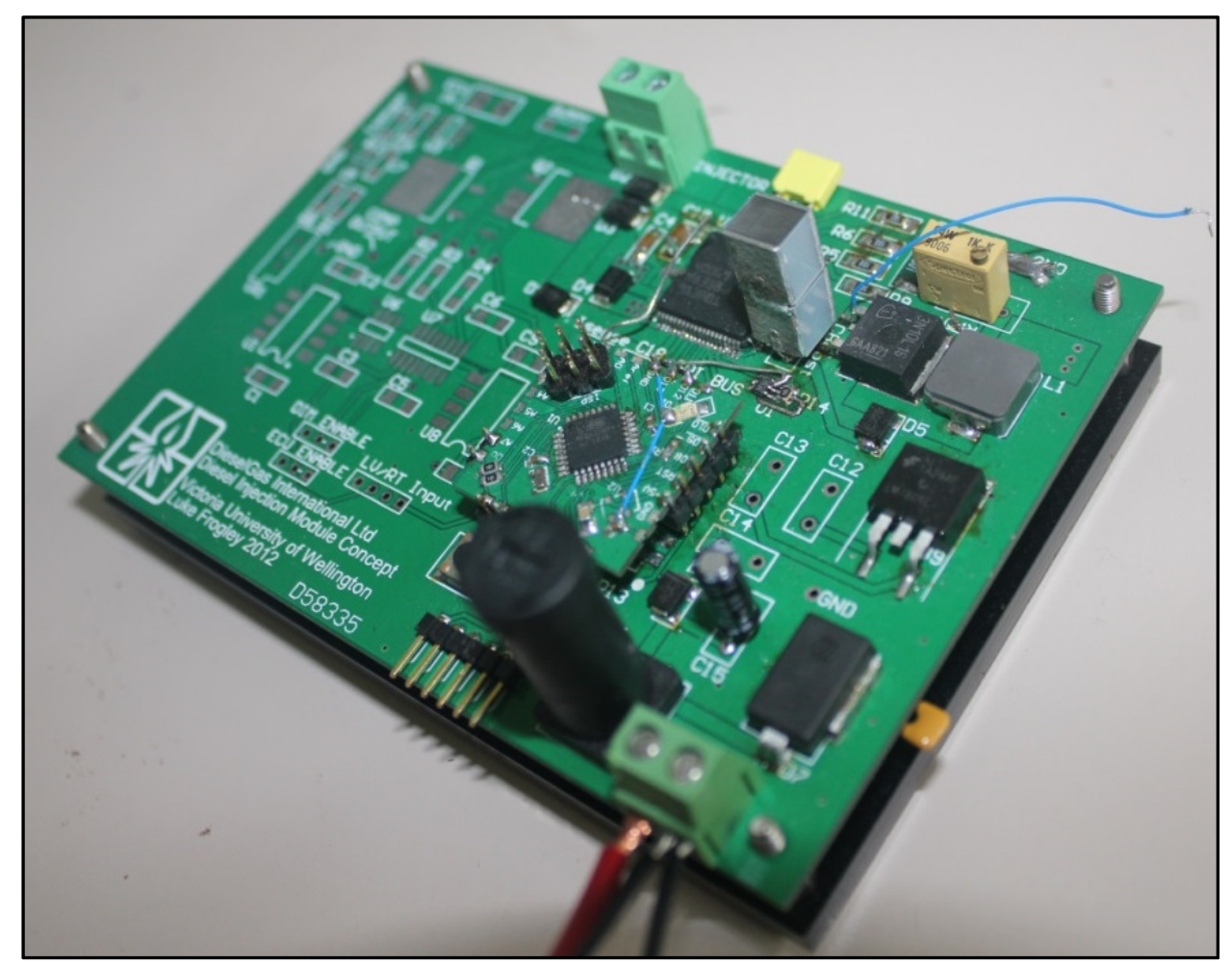

Figure 3.14 DIM proof of concept during testing

To configure the injector driver chip for correct operation, a program was written for the microcontroller to set the appropriate registers to pre-defined values upon start-up. The main values that needed modification were the clock frequency (by default at $20 \mathrm{MHz}$, needed to 
be set to $1.0 \mathrm{MHz}$ ), enabling the appropriate injector channel (all others disabled) and selecting whether or not the DC/DC boost converter is enabled.

The program written for the microcontroller initially only set these values as defaults upon start-up, but was eventually changed to allow the register configuration to be changed during operation while also displaying the contents of the diagnosis registers. The microcontroller takes commands via serial communication with a PC, and sets the internal registers of the injector driver via SPI.

The SPI communication between the injector driver and the microcontroller consists of two bytes of information; an instruction byte and a data byte. The instruction byte tell the chip what instruction to perform, such as reading or setting the value of a particular register. The data byte then provides the value to which the register shall be set (only in the case of a write instruction as data bytes are not required when reading a register). The chip then responds with a check byte and either the value of the register to be read (in the case of a read instruction) or a byte of all zeroes in the case of a write instruction.

The microcontroller takes the instructions as a hexadecimal number, and sends these to the injector driver. As a method of double-checking, the microcontroller then reads the value of the register just set, to confirm that the write operation was successful. For example, the instruction "E8 8F" will set the PARACON 5 register to "8F" (10001111) and will enable high side switch HPIV11, enable the DC/DC converter, set it to toggle on and off when required, and set the state machine to default mode. The microcontroller sends this instruction to the injector driver, and then reads the value of that register. It will then print to the serial port the value of that register for the user to verify. Additional commands were added to read the values of all registers, and also to reset the CJ841 to the default values. The code for configuring the injector driver via serial interface can be found in appendix 1 .

The injector driver chip was initially tested while being driven by a $1 \mathrm{~Hz} 0-5 \mathrm{~V}$ signal with a pulse width of $1 \mathrm{~ms}$. The first snapshot of the current waveform showed what appeared to be the correct operation, but upon closer inspection, the current output was not nearly as high as it should have been. Instead of being $~ 12$ A, it was only peaking at about $5 \mathrm{~A}$, as shown in figure 3.15. This was the first indication that the driver was not operating correctly. 


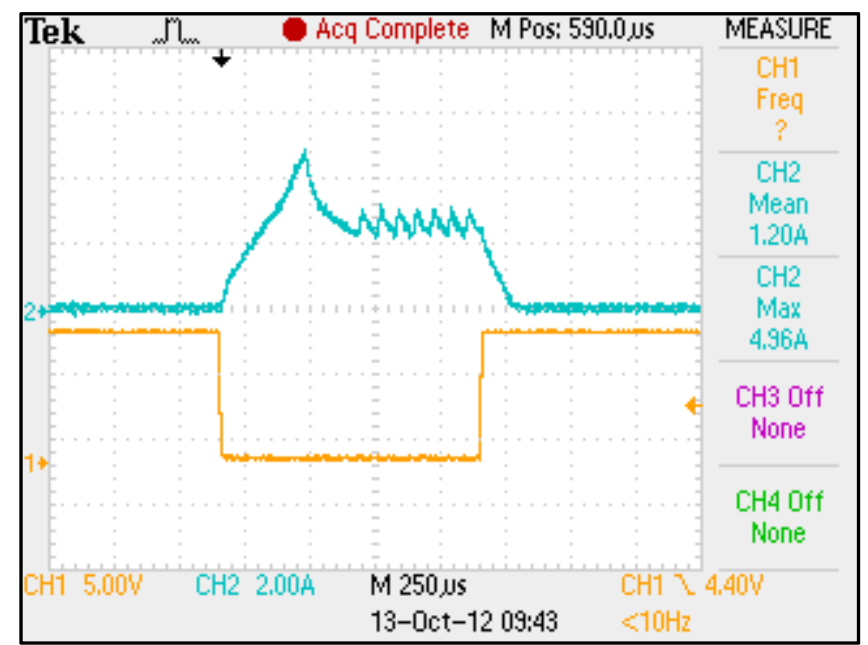

Figure 3.15 Injector driver current output

The voltage on the BOOST pin was then probed and found to be fluctuating with the pulsing of the low side switches during the holding phase of operation. This showed that the driver chip was operating as it should be, and that the BOOST voltage was not high enough to provide the high current required for the opening phase (figure 3.16). To diagnose this problem, the output from the injector driver to the gate of the FET was analysed during a boost cycle. It was found that the boost voltage was not increasing with the switching of the FET. Figure 3.16 shows the signal to the gate of the FET turning on and off (purple) and the BOOST voltage (green). As one can see, the BOOST voltage is not increasing at all with the switching of the transistor.

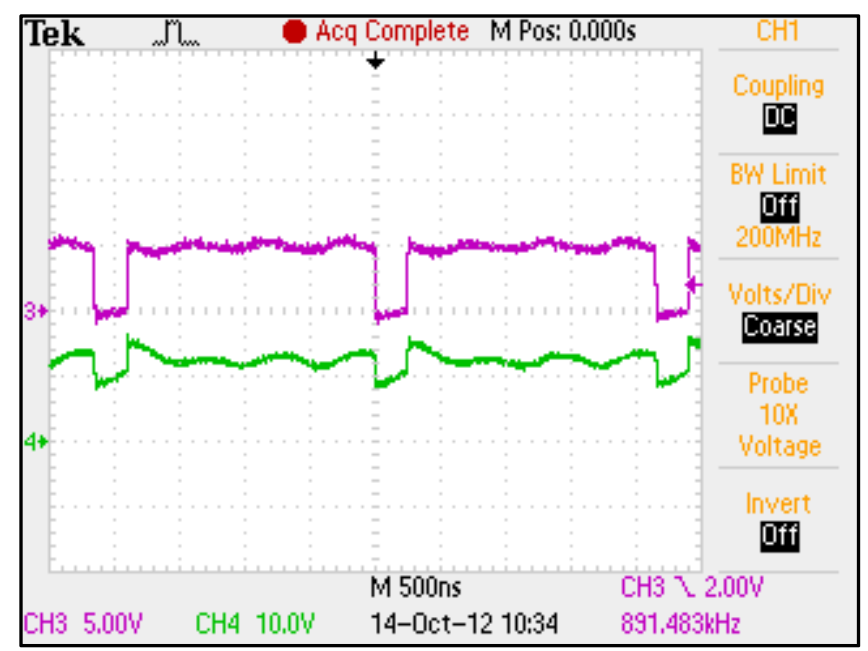

Figure 3.16 BOOST voltage (green) vs FET switching (purple)

The cause of this fault was discovered to be that the shunt resistor (required to give feedback to the injector driver) was not grounded, meaning current was not flowing through the inductor, FET, and shunt. After grounding the shunt the DC/DC boost converter began 
operating correctly. By altering the value of the feedback resistor, the BOOST voltage could be increased from $12 \mathrm{~V}$ to $\sim 100 \mathrm{~V}$. Despite the DC/DC boost converter now working correctly and providing a sufficiently high boost voltage, the injector driver would still not output the high current required to open an injector. At first, it was thought that the capacitors on the board were not large enough to provide the required current, so the capacitors responsible were swapped out for ones with a larger capacitance. The capacitors chosen were polyester film capacitors which, whilst having a higher capacitance, also have a low ESR, meaning they can supply current faster than one with a higher ESR and thus longer time constant. This did not seem to rectify the problem.

\subsubsection{Change of Concept}

During the testing phase of the proof of concept, a decision was made to abandon the injector driving method in favour of the second concept: injector gating. This decision was made after talking to an industry expert who expressed concern about the safety of the injector driving method, as detailed below.

While using an independent injector driver in the DIM is the most useful and versatile solution to the problem in that it allows full control over both fuel delivery volume and timing, there is a major flaw in having to develop fuelling tables for the engine from scratch. The biggest concern is the possibility of over-fuelling the engine, whereby the DIM injects more fuel than it should, causing the engine to speed up instead of slow down or maintain a constant speed. This poses a significant safety risk to the driver of the truck or other road users, or even equipment that may be run by a stationary engine.

Another disadvantage to using an independent injector driver is the complexity of both the hardware and the software required. The hardware is more complex due to the requirement of injector driver chips and the associated components, as well as the switching hardware and dummy loads, and the software is more complex because the DIM has to control when and how much fuel is required. The software that controls fuelling must be fully verified to ensure that it operates safely under all possible operating conditions, and will require extensive testing to verify its safe operation.

Injector gating is favoured over the injector driving method because of its simplicity and inherent safety; there is no possible way that gating the injector signals will ever deliver too much diesel to the engine. The gating method is much simpler than needing injector drivers 
to perform the diesel injection; instead, it leaves the existing hardware in the OEM ECU to do its job and takes advantage of the OEM's fuelling tables and control loops (such as those required for injector calibration, see chapter 2.1.2.3) to handle the basic fuel delivery and timing. This allows the DIM to simply use the switching hardware to steer the injector current between the injectors and the dummy loads. While the injector gating method does not have the full versatility and control of the injector driving method, it is both simpler and safer, and in a situation where the safety of others may be compromised, it is clearly the better option.

The injector driving method was chosen primarily because of the versatility and power it had over the gating method. During the initial concept phase, this was an attractive advantage before the potential flaws in safety were made apparent. The system could still be made safely with thorough testing and various fail-safes, but this would lead to a highly complex system and require more time to produce and test. As this will eventually be a commercial product, time is a significant factor for the industry partner and so the simpler method is preferable. 


\section{Second Prototype}

\subsection{Injector Gating}

The idea of injector gating was discussed in the previous chapter and was not initially chosen in favour of using the injector driving method in order to have more control over the fuelling of the engine. As mentioned in the previous chapter, after talking to an external source who has extensive knowledge of diesel injection systems, it was decided that the risks associated with controlling the fuelling ourselves were not worth the benefits of the added control. Therefore, the simpler method of gating the existing injection signals was chosen, with the added benefit of reducing the overall complexity of the project and reducing dependency on the hardware systems.

\subsection{Prototype Overview}

The gating method is simple; the current pulses from the OEM ECU are steered through either a dummy load or an injector by the DIM. The injection pulses are windowed such that any pilot injections are removed, any post injections are removed, and a shortened main pulse is allowed to pass through to the injector. In this way, only a fraction of the fuel is delivered to the cylinder, and the OEM ECU still thinks that it is driving the engine as per normal.

By using a gating method, there is no risk of accidental over-fuelling, and the complexity of the hardware is significantly reduced. No injector drivers are required as only the switching hardware and dummy loads are needed to perform fuel reduction. The only additional hardware required is control for the timing signals passing from the engine to the OEM ECU. Because there is no longer a way to control when fuel is injected outside of the OEM ECU injection pattern, it will be necessary to advance and/or retard the timing of fuel delivery to achieve optimal combustion and reduce knock.

The original specification for the DIM was to take the timing signals from the flywheel and cam shaft, condition the signals into a logic-level signal for processing, modify the signal if necessary and then output the signal to the OEM ECU with an ideal resolution of 0.1 degrees. In this way we can effectively phase-shift the ECU in relation to the engine, fine-tuning the advancing and retarding the timing as necessary. The hardware required to condition the input and output signals is fairly simple (as described in the next section), and much of the 
process is controlled by software, making development and testing much easier and faster than making hardware changes.

The block diagram for this new DIM concept is shown in figure 4.1. Instead of having an injector driver, the microcontroller simply selects which way to steer the current through the switching hardware; either through the injector or the dummy loads. A current sensing element is still used to detect OEM ECU injection angles, and to trigger the internal timers to regulate injection duration.

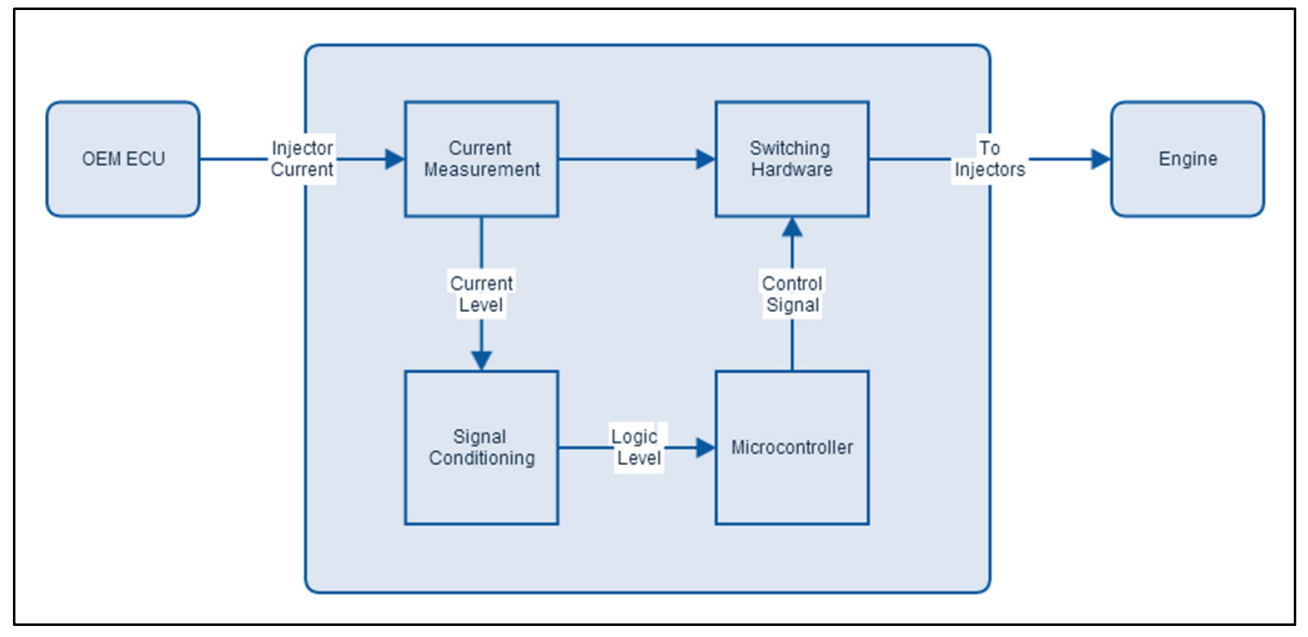

Figure 4.1 Revised DIM block diagram

The mode of operation for this DIM closely resembles the operation of the DieselGas Controller (DGC) which modifies mechanical diesel injection. The DGC would drive a stepper motor which would limit the linear movement of a rack inside the mechanical fuel pump. The DIM varies the length of a window through which current can pass. In both cases the output values are proportional to the amount of diesel delivered to the engine. The only difference is that for common rail operation, the sliding scale is done electronically in silicon as opposed to needing electromechanical devices. This again makes writing the code for the DIM much easier as the output for diesel gating is simply a time value.

The hardware for this DIM is based on the DGC, which uses an ST10 automotive microcontroller [31]. The DIM is basically a DGC but with the stepper motor outputs removed and the switching and timing hardware added. The proof of concept DIM will only control diesel injection and handle the timing advance/retard and will use a slightly modified DGC (now called a Dual Fuel Controller (DFC)) to control the other functions such as gas injection, air control, and knock detection. These functions are not new and can be replicated easily, so this proof of concept will just focus on the gating and timing systems. 
The DFC controls the DIM over a Controller Area Network (CAN) bus (an automotive bus standard found in modern vehicles and equipment), and tells the DIM when to open the injection window in terms of degrees before top dead centre (TDC), how long to hold the window open for, and also how many degrees advanced/retarded the timing signals sent to the OEM ECU should be. The DIM sends the DFC engine timing information (not phaseshifted) allowing it to determine where in the combustion cycle the engine is, which it uses to perform the knock detection.

The basic overview for this concept is shown in figure 4.2. The three main blocks of the DIM are the current detectors, switching hardware, and microcontroller. The microcontroller not only takes input from the current detectors, but also the timing signals from the engine. The timing signals are used to determine the position and phase of the engine, so the DIM knows when to open and close the injector gating window. The timing signals are also used by the DFC to detect knock on a particular cylinder. The microcontroller can also phase-shift the timing signals sent back to the OEM ECU in order to advance or retard the timing of the fuel delivery to the engine.

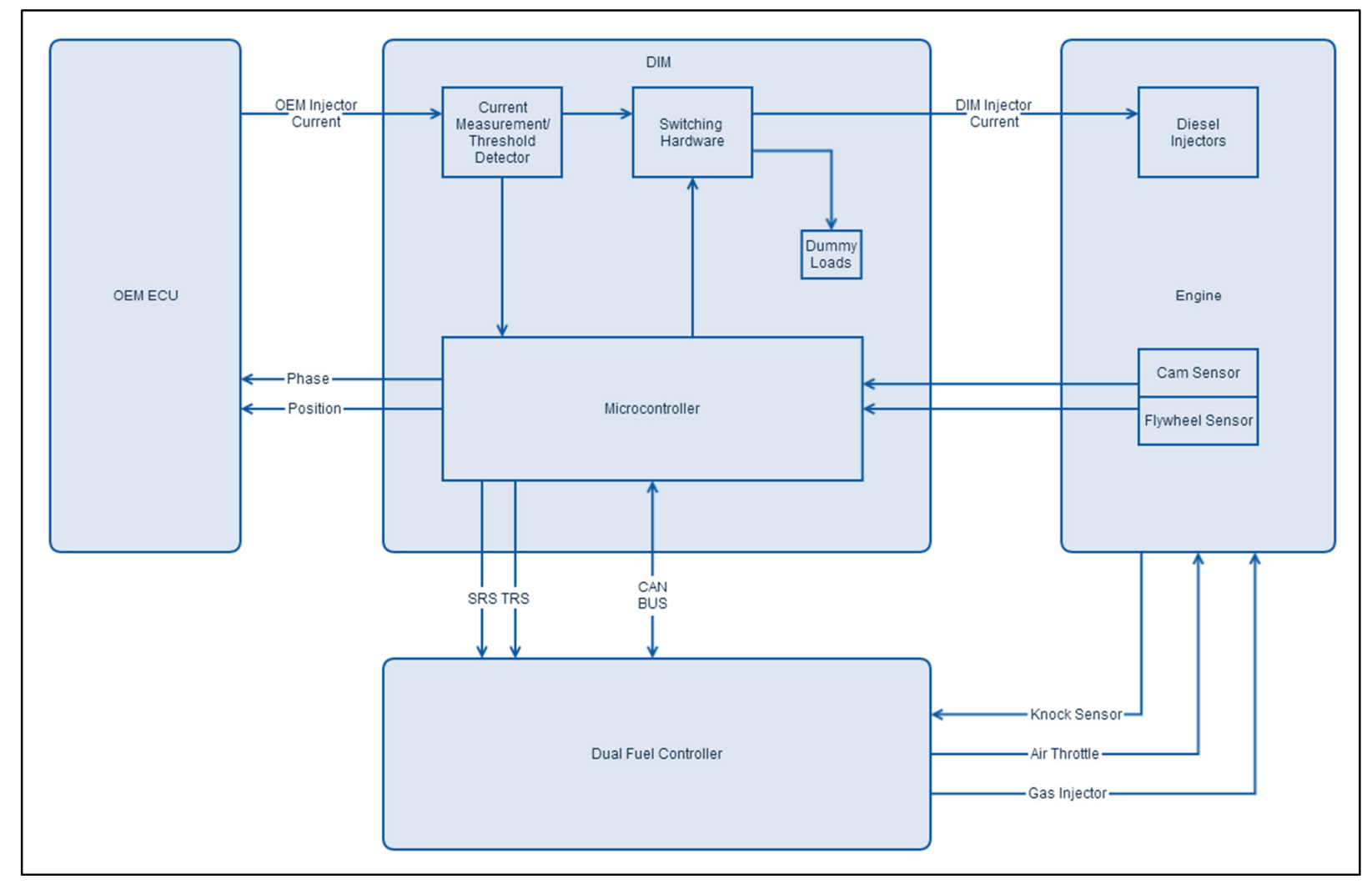

Figure 4.2 Detailed DIM block diagram showing DFC integration 


\subsubsection{Schematic}

The injector switching hardware was taken directly from the previous concept and adapted only slightly for this task. The FETs and drivers are the same, and the current sensing componentry is the same.

The main blocks in this DIM concept are:

- Injector switching hardware

- Dummy loads

- Current sensing

- Timing control

- $\mathrm{CPU}$

- Power supply

The injector switching hardware is almost exactly the same as the previous concept, but without the need to switch in an injector driver. There are only 2 FETs required to switch the dummy load in place of the real injector. A single ADUM7234 gate driver is required to drive both FETs and a single isolating power supply is used to power the gate drivers. Even though the engines available for testing are both 4-cylinder engines, the DIM was designed for implementation on engines with up to 6 cylinders, allowing testing on larger engines in future.

A schematic of the switching elements for a single cylinder is shown in figure 4.3. The INJ+ port is connected to the high side of the injector, and also to the OEM ECU high-side output. The injector current is steered through either the injector or the dummy load by turning on the respective FET. The current sensor is in series with the OEM ECU current path and is used to detect the beginning of injection.

The dummy loads for this concept are to be mounted on a PCB, and need to be designed so that they are robust and secure, while not taking up too much space on the board. The initial design of a coil of wire around a steel core worked well in testing, so a similar approach was taken for this design. Instead of using a bolt as a steel core, a steel bobbin was designed to form the core. The bobbin has a mounting hole to secure it to the PCB and with slots milled in to allow the ends of the coil to protrude into holes in the PCB for soldering. 


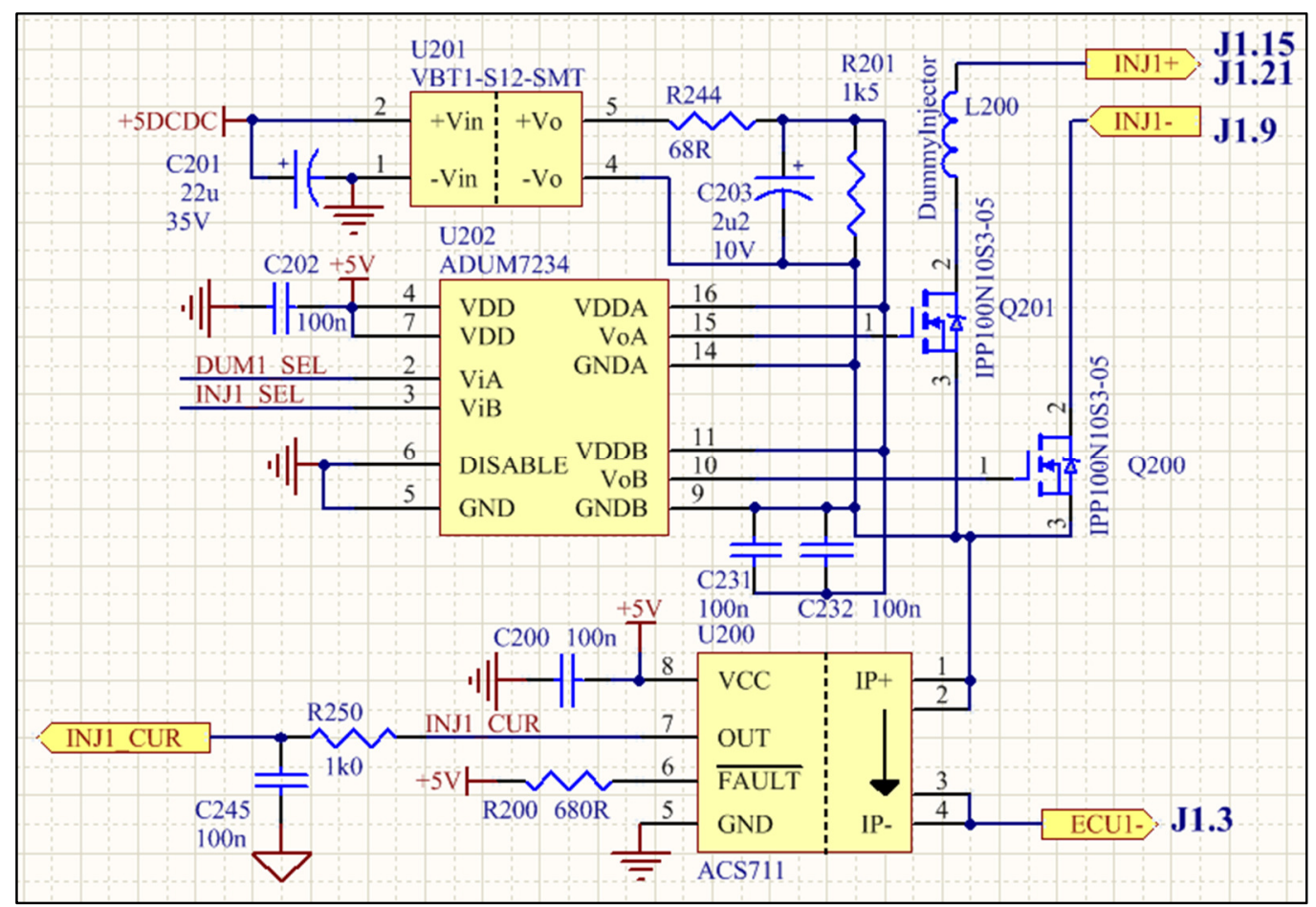

Figure 4.3 Revised switching hardware for injector gating and current detection

The dimensions of the bobbin are limited by the height of the DIM enclosure, and are restricted in diameter so as to minimise the amount of space taken up on the PCB. A figure of $16 \mathrm{~mm}$ outside diameter was chosen, and the inner core was decided to be $13 \mathrm{~mm}$ to allow a sufficient number of turns. The bobbins are designed in Solidworks and manufactured out of mild steel. The bobbins were then powder coated to prevent corrosion and also to insulate the steel from shorting to the coil should the enamel wear off the wire through rubbing against the bobbin. A finished bobbin before the turns are added is shown in figure 4.4.

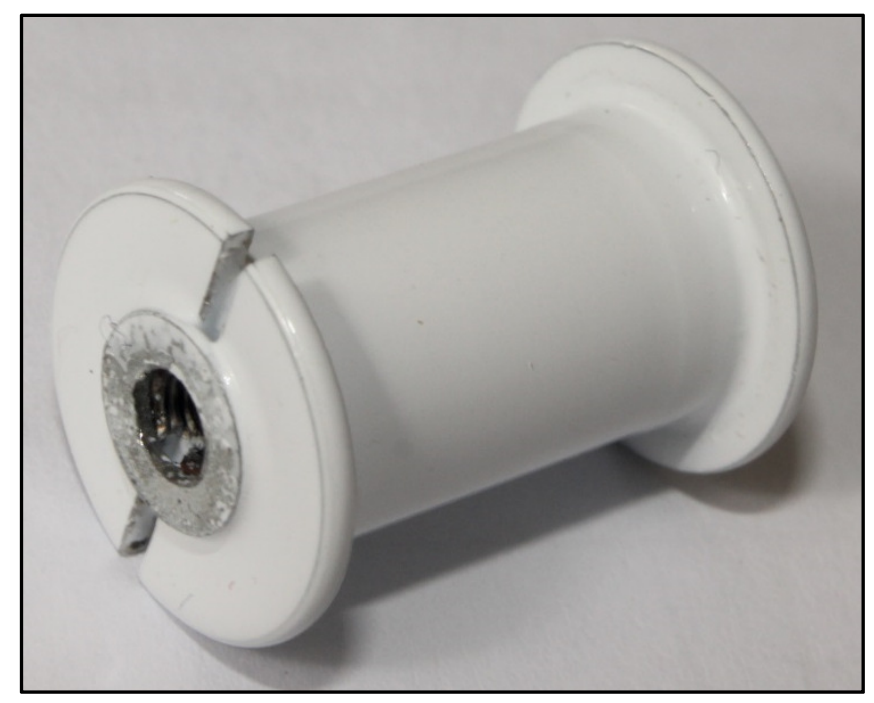

Figure 4.4 Manufactured injector bobbin after powder coating 
Timing signal detection was performed by using a comparator for the cam shaft sensor and a zero crossing detector for the flywheel sensor. The cam shaft sensor produces a digital signal which can be detected by using a simple comparator with a defined threshold, and the flywheel sensor produces an analogue signal which swings between approximately $\pm 20 \mathrm{~V}$, so a zero crossing detector is used to detect the rising edge of this waveform as it passes through $0 \mathrm{~V}$. The hardware for these components is based on the old DFC and uses MAX 942 high speed comparators [32] (figure 4.5).

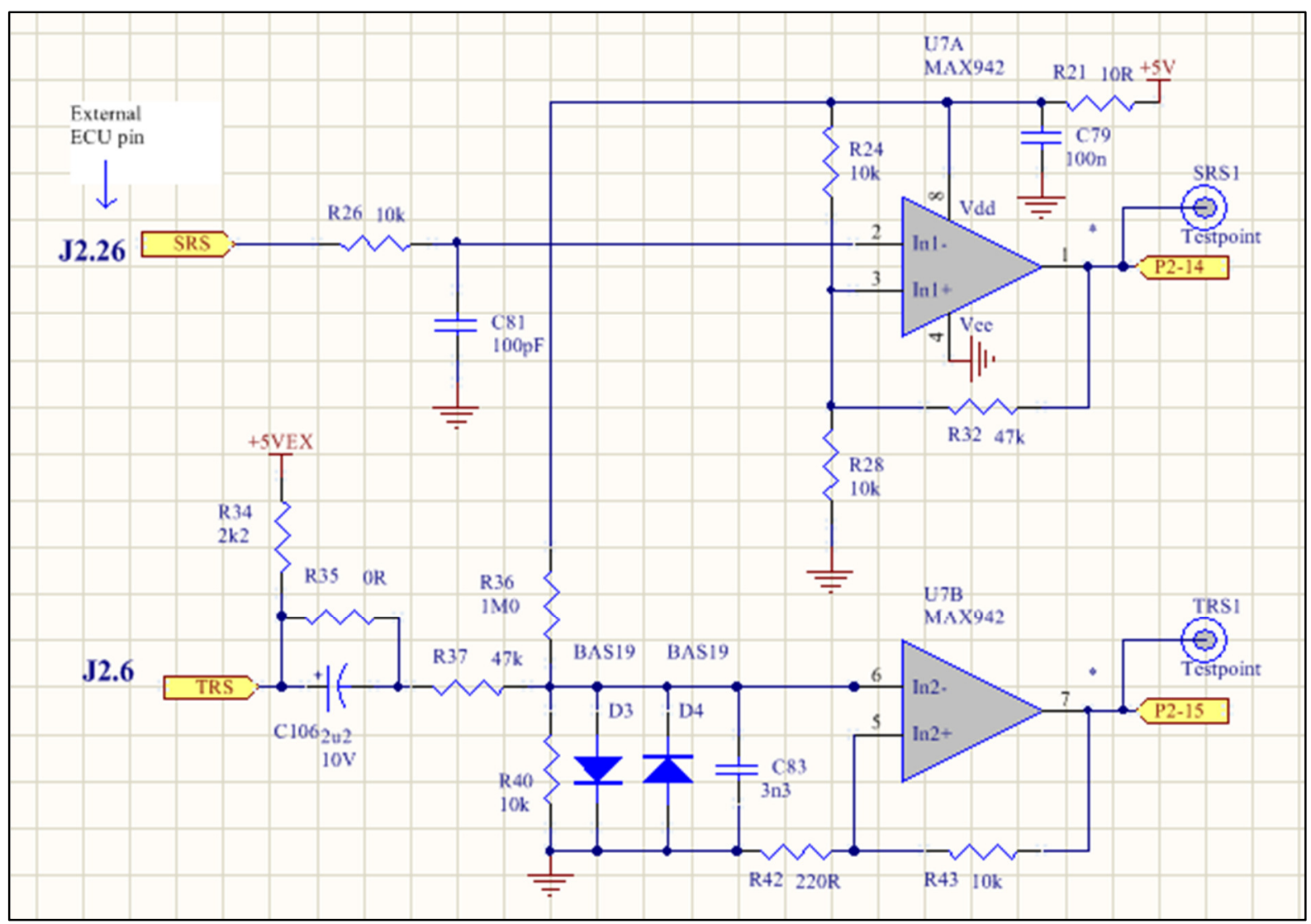

Figure 4.5 Timing signal input and conditioning

The timing outputs from the CPU are fed through a buffer, before being sent to an external pin to connect to the OEM ECU. Both of these outputs are logic level outputs (0-5 V). The DFC also receives a synchronous timing signal for it to determine the position and phase of the engine.

The CPU for this DIM is the same as that previously used on the DFCs, an ST10 microcontroller. This decision was made because it makes writing software and hardware easier as there are already a large number of custom libraries written by DieselGas International and most of the supporting hardware is already pre-tested. By using a system 
that has already been built and tested for this application, the risk of errors in the CPU layout was reduced.

The power supply for the DIM is also based heavily on the old DFC design, and is in fact made simpler by not needing on-board power supplies for the stepper motors. The power supply provides clean $5 \mathrm{~V}$ DC for all the componentry on the PCB. The isolating power supplies for the gate drivers are changed to ones that run on $5 \mathrm{~V}$, but still output a $12 \mathrm{~V}$ output [33], eliminating the need for a dedicated clean $12 \mathrm{~V}$ source in the main power supply design.

\subsubsection{PCB}

The layout of this PCB is again based around the DFC as the main CPU components and power supply remain the same. The stepper motor controllers and other sensory inputs are replaced by the injector switching system, the dummy loads, the current sensors and timing signal conditioning. Because of the size of the FETs and the size of dummy loads, it is important to design a layout configuration which will minimise the area these components will occupy. The dummy loads are placed very close together and the current sensors are placed under the FETs which stand above them. The power supplies are placed on the underside of the board to reduce congestion on the top side, but this adds an extra manufacturing step in terms of production.

The PCB is designed to be of the same size and shape of the previous DGCs, allowing it to fit in the same housing and use the same external connector. The PCB is laid out by the same contractor that produced the layout for the DGC also, in order to reduce the turnaround time for production. At this stage in the project, time was a critical factor due to the recent change in the mode of operation.

\subsubsection{Testing}

The DIM was initially produced for implementation on the BT-50 engine, but during the design phase an Isuzu 4HK1 engine was acquired and so it was decided to test the DIM on this engine instead as it is closer in size to the eventual target engines found in highway trucks. However, since the $4 \mathrm{HK}$ had not yet been mounted and run, it was only assumed that the engine signals were the same as the BT-50. This was found to only be partially true as the timing signals for each engine were subtly different. 
The first major difference seen was the output of the cam shaft position sensor. While the signal resembled the BT-50 signal somewhat, there were two major differences. Despite looking like a digital waveform with square pulses, the signal was very noisy, and there were many more pulses than on the BT-50, as shown in figure 4.6. The reason this engine has more cam shaft pulses is because this engine can run solely using the cam sensor in the event of a flywheel sensor fault. That is, if the flywheel sensor fails, the OEM ECU can still operate based on the information from the cam sensor alone. This is a useful fail-safe system for an engine used to run heavy machinery.

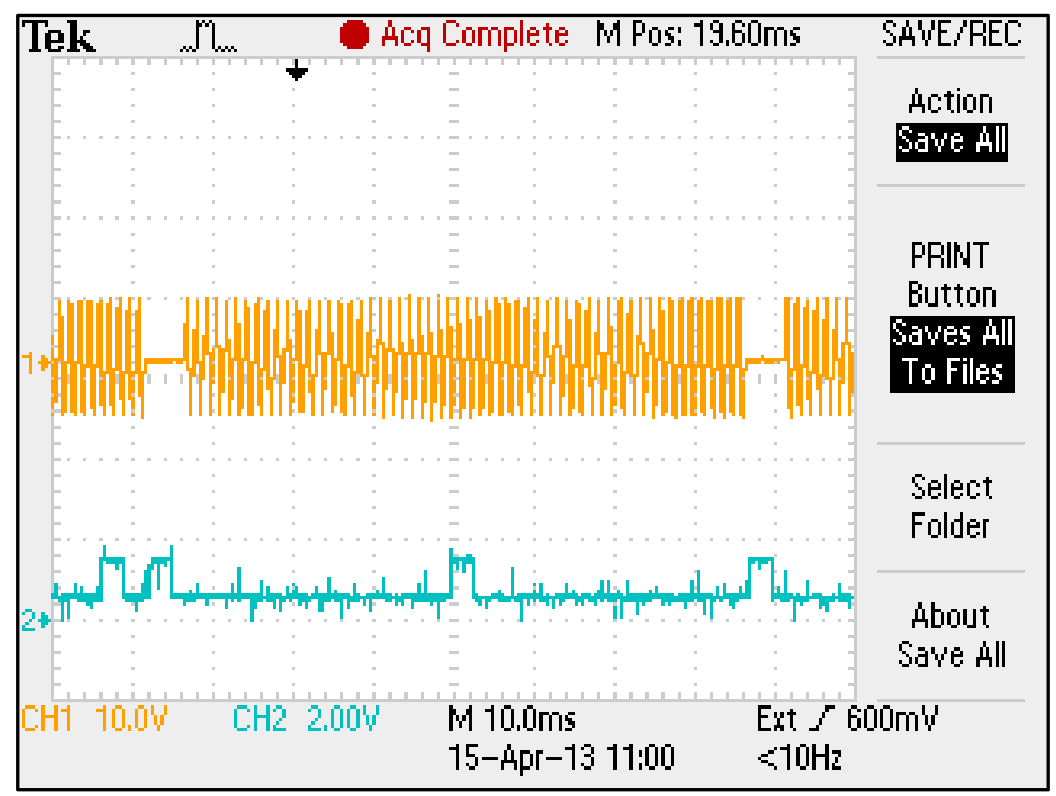

Figure 4.6 4HK1 engine cam shaft (blue) and flywheel (orange) sensor outputs

The additional pulses do not matter in terms of DIM operation, as they will be passed through to the OEM ECU, but the noisy waveform proved difficult for the comparator on board the DIM to handle. The voltage of the cam signal was only between 1 and $1.5 \mathrm{~V}$ and the noise was above and below these thresholds. It was initially thought that the sensor itself was faulty, and so the sensor was removed and tested on the bench. The sensor produced the same output. It was then thought that perhaps the sensor was not a voltage source sensor, but possibly a current source which requires a load across the output pins to pass current through (thus producing a voltage across the load.). Because there was no available documentation detailing the output characteristics of the sensor, the current source theory had to be confirmed experimentally. 


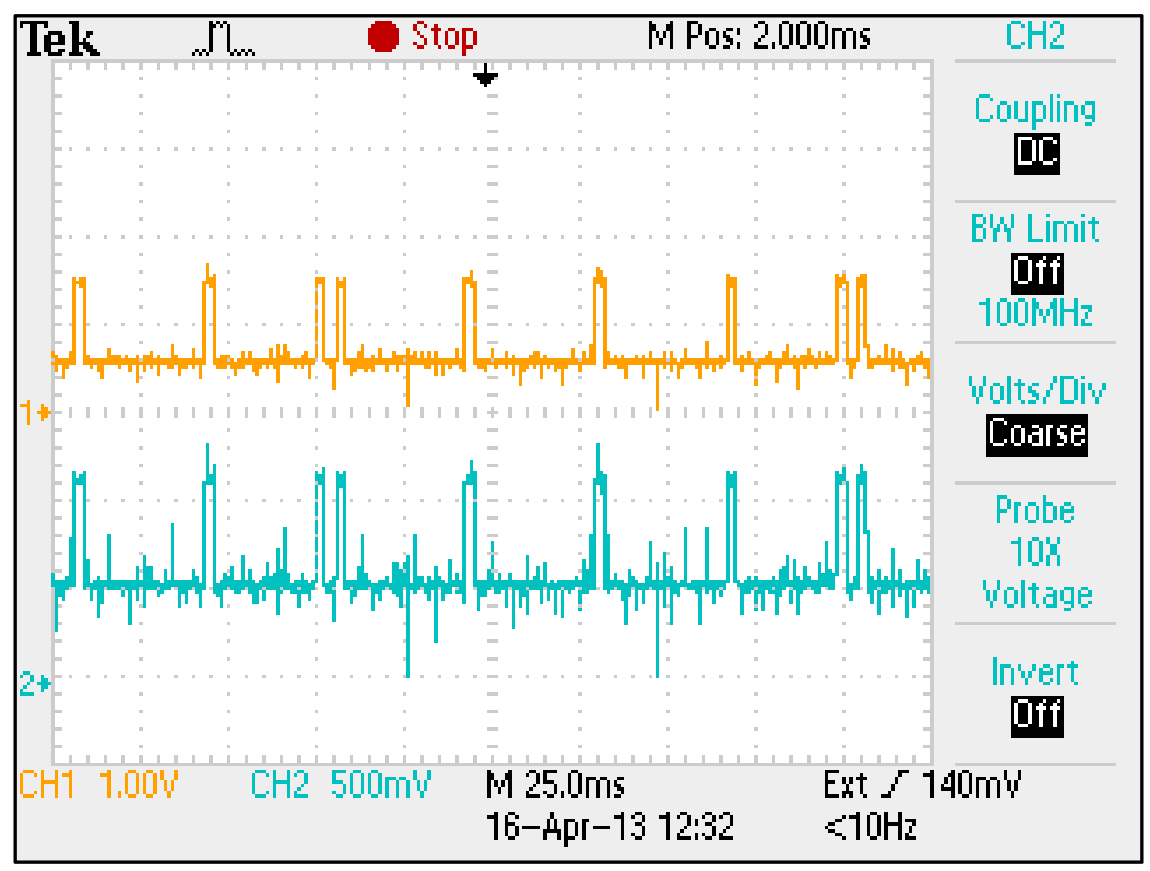

Figure 4.7 Cam shaft (phase) sensor waveform with (orange) and without (blue) sense resistor

The internal resistance of the sensor was measured and found to be $100 \Omega$. A $100 \Omega$ resistor was then placed across the output and moved across a hole drilled in a section of flat steel. The waveform from this was much cleaner, and so the sensor was placed back in the engine and the voltage across the resistor was checked with the engine running. The result is shown in figure 4.7. The blue trace shows the noise in the signal, and the orange trace shows the reduction in noise when measured across a $100 \Omega$ load.

The comparator designed to analyse the output of the BT-50 cam sensor had to be changed to take the output of the cam sensor of the 4HK1. To do this, a sense resistor was added (across which a voltage is produced when the cam sensor goes HIGH). This voltage is fed into the input of the comparator. The threshold value was then changed to lie between the 1 and $1.5 \mathrm{~V}$ signal. With these changes, the comparator now outputs a logic level signal in phase with the cam sensor.

The signal that the OEM ECU expects is no longer a 0-5 V digital logic signal, but a current source. Therefore, an output stage had to be created to deliver the correct signal. This output stage needs to output a current of $\sim 100 \mathrm{~mA}$ in reaction to a logic signal from the CPU. A simple current source was produced with a FET and two resistors (figure 4.8) to provide the correct current output. 


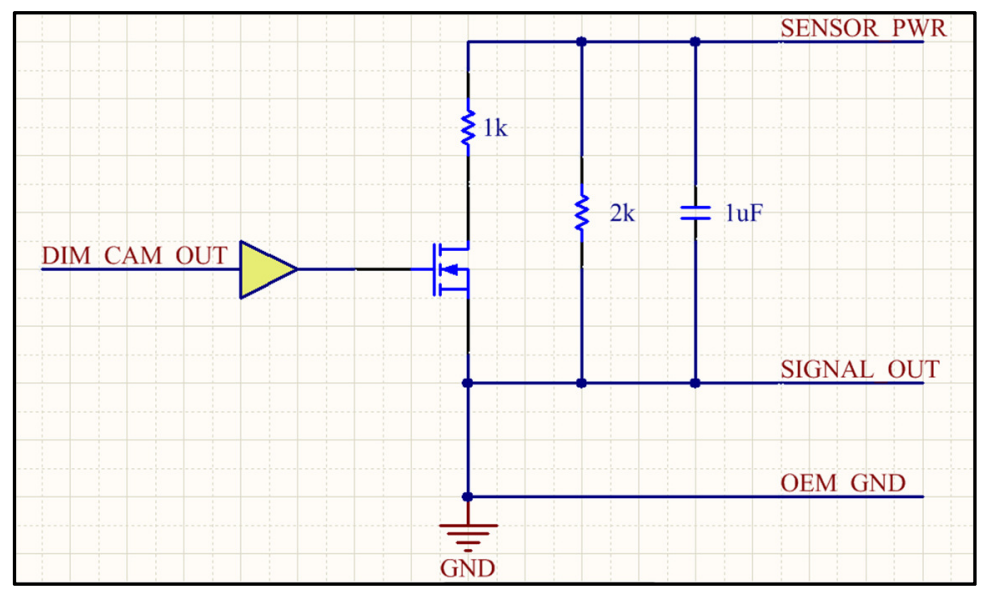

Figure 4.8 Phase signal output stage

The gate of the FET was connected to the CPU and the output of the current source connected to the OEM ECU (figure 4.8). The output of the DIM was then visually compared to the output of the cam sensor to confirm they were suitably similar and in phase, as shown in figure 4.9. The orange waveform is the output from the sensor itself and the blue trace is the output from the DIM to the OEM ECU. The output from the DIM was plugged into the OEM ECU and the engine ran smoothly, confirming that the output signal was indeed sufficient for engine operation.

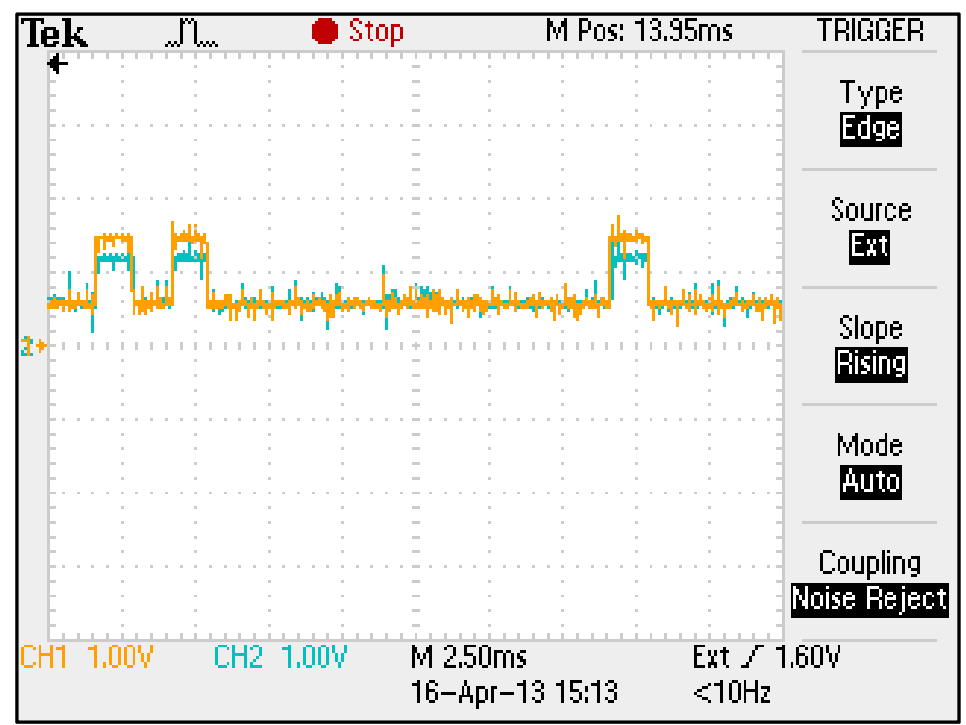

Figure 4.9 Phase signal input from engine and output to OEM ECU

The flywheel sensor output is fed into a zero crossing detector which detects the rising edge of the flywheel sensor signal as it passes from a negative to positive voltage. To ensure the DIM and DFC know where in the cycle the engine is, this signal must be in phase with the output of the flywheel sensor. However, in initial testing it was found that the sensor had 
been connected backwards and was passing incorrect information to the DIM (figure 4.10). This was not easily identified initially, as the engine would still run but would surge in power. This behaviour was initially misdiagnosed as a mechanical fault, but upon further inspection it was discovered that the issue was with the flywheel signal and transposing the wires of the flywheel sensor input resolved the problem.

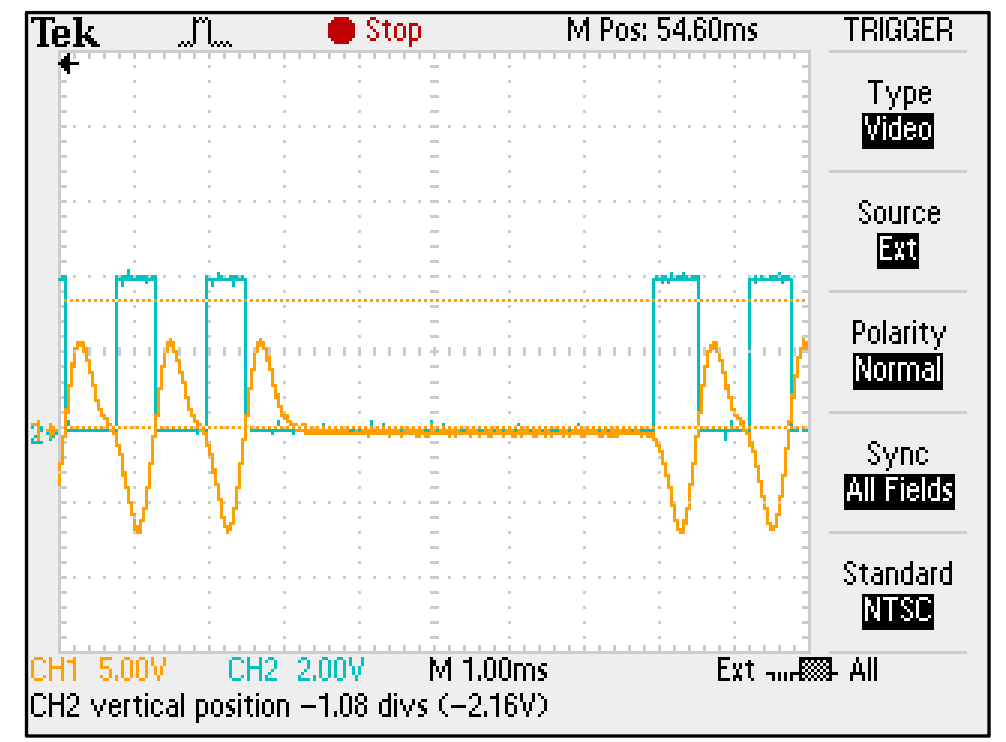

Figure 4.10 Flywheel sensor before and after input stage (out of phase)

The output from the CPU was initially a logic level signal which was fed through to the OEM ECU. The output was passed through two series capacitors to remove the DC offset and to make the output floating. However, this did not work, and so the output was then passed through a transformer, isolating the output of the DIM from the OEM ECU. Unfortunately, because of the square wave signal, the transformer would output large inductive spikes in voltage, so this method was dismissed.

It is assumed the OEM ECU also uses a zero-crossing detector to detect the flywheel teeth, so all that is needed by the OEM ECU is for the output signal to pass through $0 \mathrm{~V}$. Therefore the signal can simply be shifted down $2.5 \mathrm{~V}$ and retain the same square waveform (as it does not need to be sinusoidal). In the end, a simple level shifter run off an external battery was used as a temporary solution and the output signal, shown in figure 4.11, was able to drive the OEM ECU. This level shifter was produced on veroboard and mounted externally, and will be incorporated in the next hardware revision. 


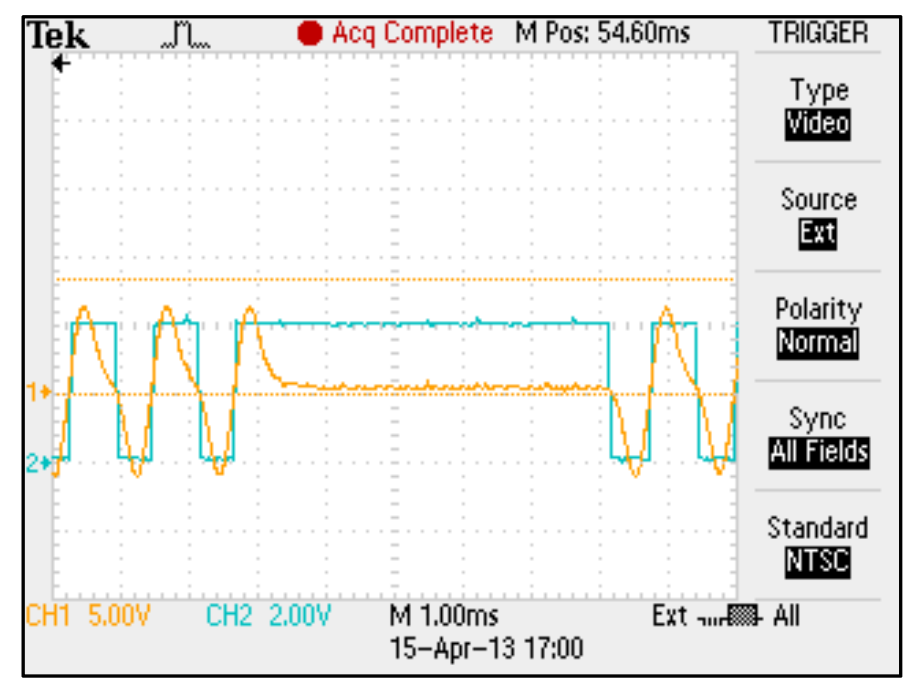

Figure 4.11 Flywheel sensor before and after input stage (in phase)

On the DIM, an LED is used to indicate when the injection pass-through window is open. When this LED is off the injection pulses are steered through the respective dummy load. Under normal pass-through conditions (diesel mode), all the LEDs are constantly on as all injection pulses are sent to the injectors. When running in dual fuel mode, the LEDs will be flashing, indicating the opening and closing of the injection windows. This can show, at a glance, whether or not the DIM is in dual fuel mode and if it is operating correctly.

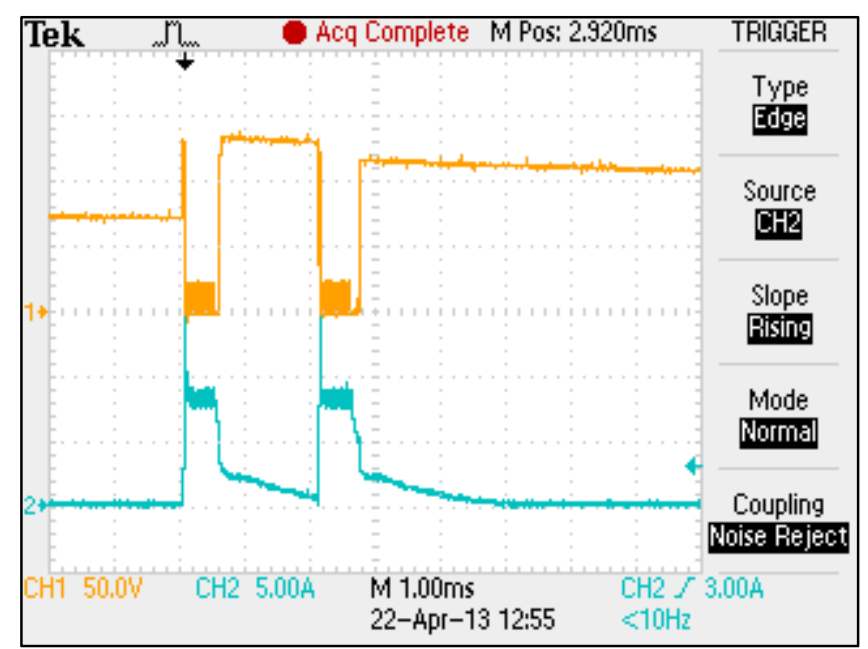

Figure 4.12 Shortened injection pulses (blue) slowing current tailing off slowly

One key difference that was noticed during testing of the 4HK engine, was the shape of the injector current waveform when the DIM was connected. When the OEM ECU is connected directly to the injectors, the injection waveform appears as the blue trace in figure 4.13. However, when the injector lines were connected via the DIM, the waveform changed slightly to look more like the blue trace in figure 4.12. The cause for this was not 
immediately evident as the engine would still run and function normally, but the injector current would tail off slowly instead of the sharp cut-off expected. The cause of this behaviour was found to be due to the intrinsic diodes in the FETs, which were allowing the negative voltages induced by the injector solenoids and dummy loads to resist the sudden shut-off of current flow. While there is no immediate remedy for this issue in the current hardware revision, it is likely that using a slightly different switching method such as a solid-state relay (SSR) will fix this problem. This will be discussed in the future work section.

Despite the injector current waveform tailing off slower than normal, the injection pulses are definitely seen to be reduced in length. Figure 4.13 shows the comparison of the OEM injection pulses (blue) and the gated signal (orange) passing through to the injectors. This figure illustrates how the pilot injection pulse is removed by the gating system. Removal of this pulse indicates a reduction in diesel delivered to the engine, and thus a reduction in output power. To test this operation further, a set window angle and injection duration were selected such that the engine was observed to reduce in power, and then the demand signal to the engine was increased.

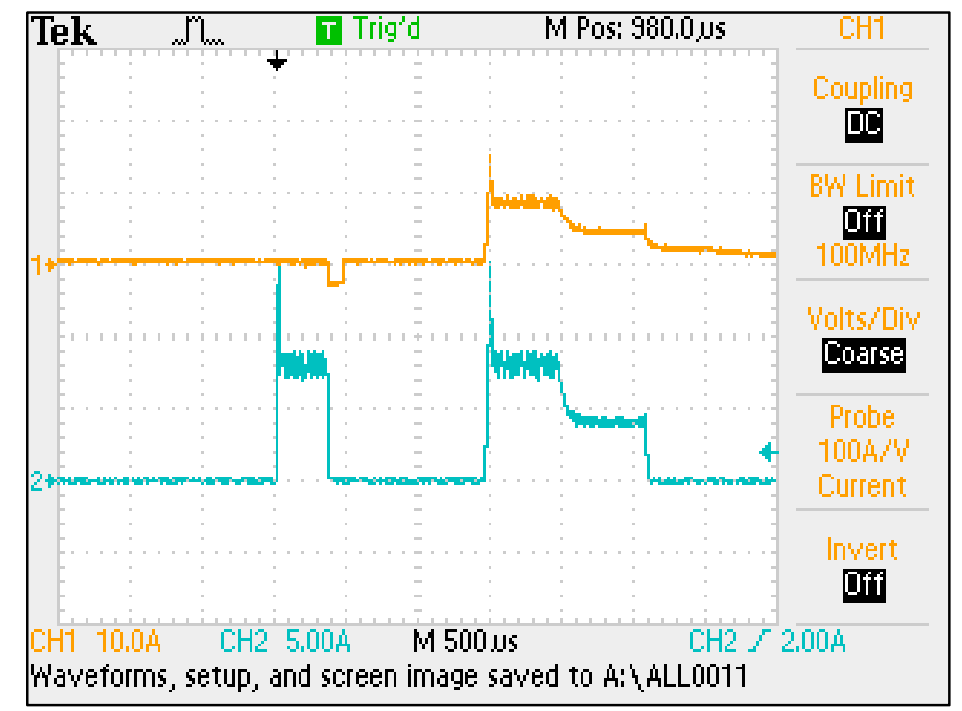

Figure 4.13 OEM injection pulses (blue) and reduced injection pulses from the DIM (orange)

Despite increasing the demand on the engine to $100 \%$, the engine would not accelerate as the amount of fuel delivered by the injectors was fixed due to the set injection duration allowed by the DIM. This proves that the injection gating system can effectively control the diesel delivery, and is suitable for implementation in a dual fuel system. 


\subsection{Test Equipment}

As well as the hardware for the DIM, other items have been designed for testing purposes in order to evaluate the performance of the DIM and to analyse the performance of the engine itself.

\subsubsection{Pressure Transducer}

One way of measuring how well the engine is running is to analyse the combustion pressures inside the cylinder. This is achieved by using a pressure transducer rated for such high pressures and temperatures, and mounting it in the cylinder head. By analysing the pressure waveform one can determine how soon before or after TDC the fuel is ignited, and how flat or peaked the pressure curve is. This gives an indication of how efficiently the engine is running.

Diesel engines are easy engines in which to install pressure transducers because they have glow plugs. Instead of needing to drill a hole to install a transducer in the cylinder head, it is possible to instead produce an adapter which will fit in the glow plug socket, allowing the transducer to measure the cylinder pressures via the glow plug port. This approach was taken for the BT-50 and the 4HK engines. The BT-50 glow plug adapter was designed and manufactured by a third party at a cost of approximately $\$ 500$. The $4 \mathrm{HK}$ adapter was designed in-house and was manufactured by the Victoria University workshop for approximately half this cost.

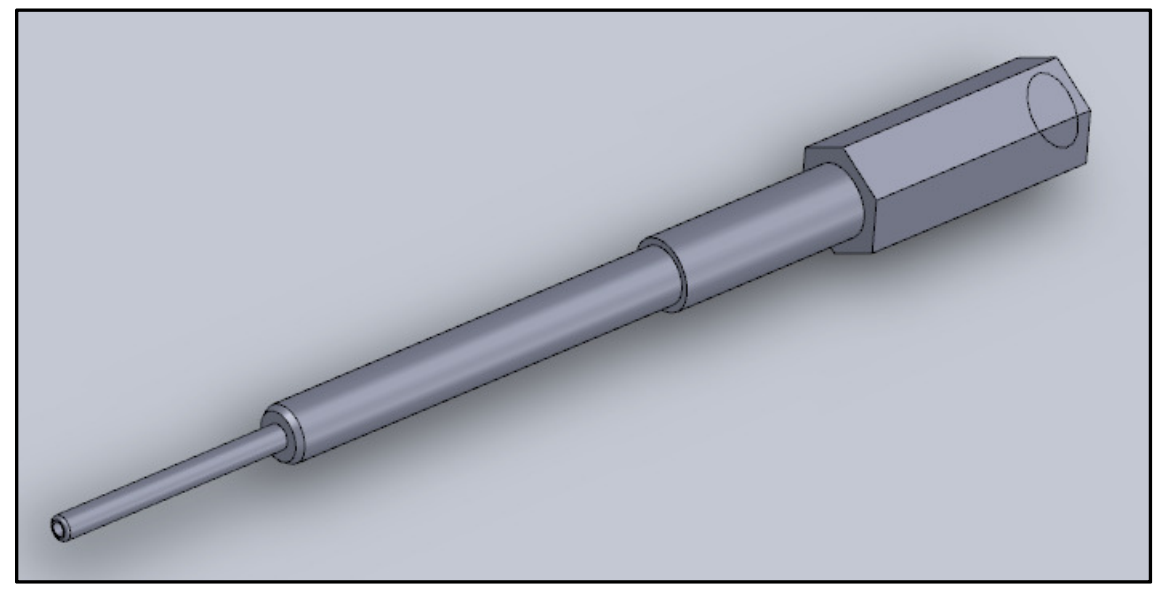

Figure 4.14 Glow plug adapter for pressure transducer

The glow plug removed from the $4 \mathrm{HK}$ was measured and a suitable adapter (seen in figure 4.14) was designed in Solidworks with the correct mounting dimensions for the pressure 
transducer, as sourced from the datasheet. The mounting specifications for the pressure transducer [34] were very specific so as to produce accurate readings and to minimise damage to the sensor body.

The pressure transducer used in this project is a Kistler 6123, non-cooled quartz pressure sensor. This is the pressure sensor previously used by DieselGas in the development of earlier dual fuel projects. The range of this sensor is 0-200 bar, with a sensitivity of - $16 \mathrm{pC} / \mathrm{bar}$. A charge amplifier is used to amplify the signal for analysis.

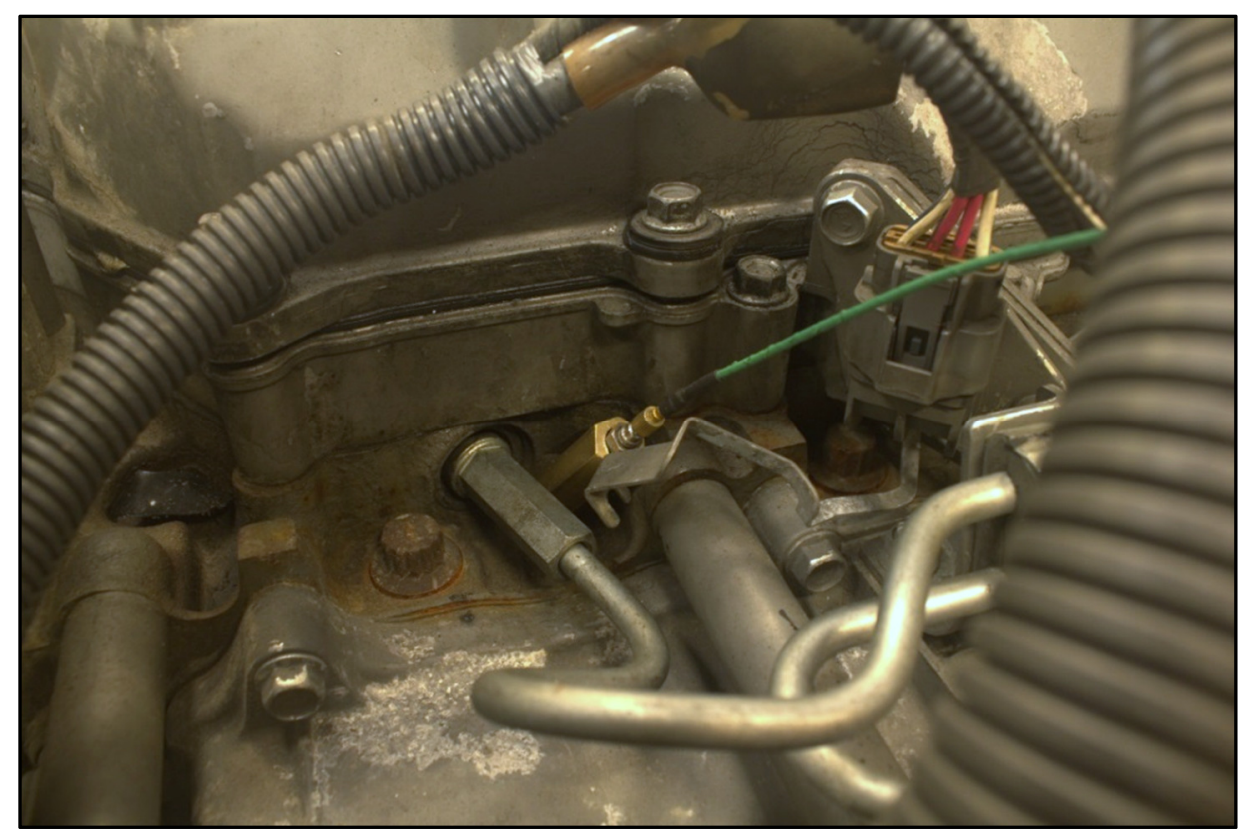

Figure 4.15 Pressure transducer installed in cylinder head

The pressure transducer mounted in the glow plug (figure 4.15) adapter sends a signal to a charge coupled device, which is monitored by a PC with a fast analogue-to-digital (ADC) card, and displays a pressure trace onscreen (discussed further in chapter 5.1.5). Analysis of the pressure trace can determine how many degrees before/after TDC combustion occurs and how high the cylinder pressure gets. This gives a rough indication of what is happening inside the cylinder and can help determine how the system needs to be altered to run more efficiently.

\subsubsection{Engine Simulator}

Because the software development was performed by a developer offsite, it was necessary to send him a DIM to work on and program. However, since he did not have access to an engine to test on, an engine simulator was designed to allow him to test the software on the test 
bench. The engine simulator is a microcontroller which emulates the $4 \mathrm{HK}$ engine by producing the timing signals required for DIM operation. The two signals produced are the cam and flywheel signals which the DIM uses to determine the position and phase of the engine in order to control injection timing.

The microcontroller used is an Arduino Uno which takes inputs from either a serial port or from a potentiometer, and produces 2 pulse trains emulating the cam and flywheel sensors at a particular RPM. It was important to ensure that the flywheel and cam pulses are at the same point in the cycle as the engine and in the correct phase.

To test the engine simulator, the output signals of the engine simulator were compared to the output of the comparators on the DIM. Figure 4.16 shows this comparison. These signals are virtually indistinguishable from one another, and any variation from the actual engine signals was measured to be approximately $10 \mu \mathrm{s}$, less than 0.1 degree difference. These signals are therefore similar enough to allow the DIM to be tested using the engine simulator. The engine simulator is used by the offsite software engineer to write code and test it with the simulated signals before submitting the new firmware revisions to be tested on the ECU.

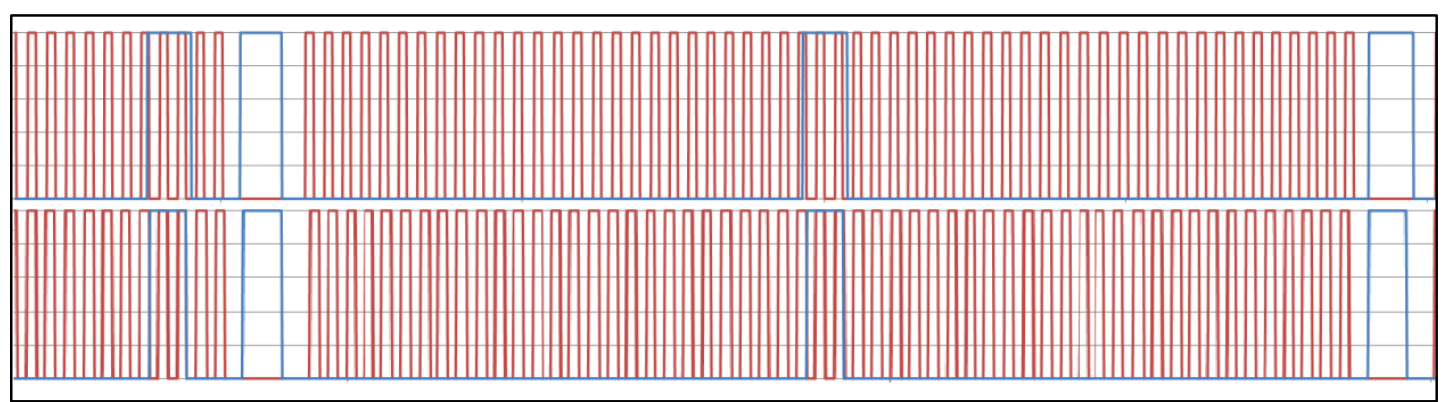

Figure 4.16 Comparison of engine (top) and engine simulator (bottom) signals, post-signal conditioning

\subsection{Software Development}

The majority of this project focused on hardware development, as most of the software was written offsite by a third party. However, there were a few situations where software or algorithms were planned out in-house.

\subsubsection{Signal Generator}

The engine simulator is effectively a signal generator which produces two pulse trains representing the output of the timing signals from a diesel engine. These signals (from the 
cam and flywheel sensors) provide information about where the engine is in its cycle. The signal generator was produced to allow an offsite software developer to test the DIM on a test bench without having to plug it into an engine. A third signal was added later to simulate an injection pulse. This pulse allowed the software developer to test the injector gating system.

The two timing signals from both the BT-50 and 4HK engines were analysed to establish the number of pulses in each cycle and determine how the two signals are related. The cam shaft and flywheel are mechanically linked in an engine (i.e. the relationship between the two signals is fixed at all speeds), and the full timing pattern (both cam shaft and flywheel signals) repeats for every two revolutions of the crankshaft. In both engines, the flywheel sensor produces pulses every 6 degrees of rotation as the timing projections pass the sensor. In both cases there are a number of 'missing' timing projections where there are no pulses present. This gap serves as a reference for the ECU and signals a new revolution of the crankshaft.

The cam shaft turns at half the speed of the flywheel, so one rotation of the cam equals two revolutions of the crank. Therefore, the cam shaft provides phase information to the ECU, and the flywheel provides finer position information as it has a higher resolution. To generate the correct signals from the simulator, the relationship between the flywheel pulses and cam shaft pulses was observed on an oscilloscope and saved to a file for analysis. The main pieces of information required were:

- How many pulses are missing from the flywheel signal?

- How many cam shaft pulses are there and, on what flywheel pulse do they occur?

This information is then fed into the Arduino which generates the pulses required. This is accomplished by dividing up the period of rotation into 60 (i.e. one count for each 6 degree projection on the flywheel) then counting through the projections and pulsing the flywheel and cam outputs as dictated by that tooth. The code for this program can be found in appendix 2.

\subsubsection{Timing Algorithm}

Because the injector gating system can only modify existing injection pulses, if the injection needs to be earlier or later in the cycle, there is no easy way to advance or retard the injection. Since the OEM ECU is reacting to the inputs from the cam and flywheel sensors, if these are 
phase-shifted relative to the actual position of the engine, then the injection pattern will shift accordingly. While it is easy to retard the injection pattern by simply adding a delay to the input signal, advancing the timing requires more computation.

In diesel mode, there is no timing advance and the DIM simply mirrors the input from the flywheel sensor. In this case, the period of the DIM output from the pulses $\mathrm{T}_{\text {out }}$ are equal to the input pulses $\mathrm{T}_{\mathrm{in}}$. To advance the timing, the pulses sent to the OEM ECU from the DIM must arrive before they are generated by the flywheel sensor. Therefore, the DIM has to predict what the engine is going to do based on past information.

A simple way to implement this is to consider the last two pulses from the flywheel and infer from that information when the next pulse should occur and how long it should be. Figure 4.17 shows how the input (blue) and output (orange) signals look when phase shifted. The blue trace is the signal from the OEM sensor, and the orange trace is the DIM output. It can be seen that the DIM output leads the sensor input by approximately $1.2 \mathrm{~ms}$.

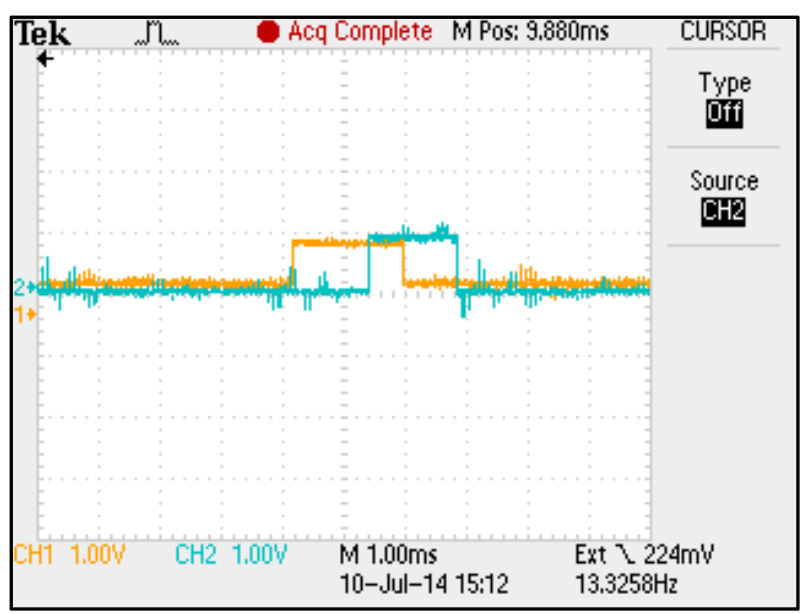

Figure 4.17 Phase shifted CAM signals during testing

In order to advance or retard the timing by a particular angle, the signals must be shifted by a particular time factor which will vary depending on the speed of the engine. For example, a 10 degree shift at idle will be a longer time shift than advancing by the same angle at full speed.

Since the period of each input pulse $T_{\text {in }}$ corresponds to 6 degrees of crankshaft rotation (as the flywheel ridges are 6 degrees apart), dividing this period by six gives a value of seconds per degree of rotation at that speed. Multiplying this value by the advance angle $\alpha$ gives the 
delay in seconds required to reduce the period of the output pulse $\mathrm{T}_{\text {out }}$ by $\alpha$ at that speed, as demonstrated in equation 4.1.

$$
\Delta=\frac{T_{\text {in }}}{6} \times \alpha
$$

(Equation 4. 1)

The period of the output pulse $\mathrm{T}_{\text {out }}$ is reduced by this delay factor $\Delta$, thus advancing the beginning of the next pulse. If the engine speed remains constant, the shortened output pulse will have advanced all subsequent pulses by $\Delta$, and the DIM can produce output pulses with the same period as the previous input pulses (equation 4.2).

$$
T_{o u t_{n}}=T_{i n_{\mathrm{n}}}-\Delta
$$

(Equation 4. 2)

However, should the engine speed change, the time shift $(\Delta)$ required to advance the timing by $\alpha$ must change proportionally. Therefore, the next $\Delta$ must be subtracted from the previous $\Delta$ to account for the change in speed. Equation 4.3 shows how $\mathrm{T}_{\text {in }}$ and $\mathrm{T}_{\text {out }}$ can be calculated for $T_{\text {in }}>3$. The timing advance system requires at least two flywheel pulses before it can begin dynamic manipulation of the timing signals.

$$
\begin{array}{ll}
T_{\text {out }_{1}}=T_{\text {in }_{0}} & \Delta_{0}=0 \\
T_{\text {out }_{2}}=T_{\text {in }_{1}}-\Delta_{1} & \Delta_{1}=\frac{T_{1}}{6} \times \alpha \\
T_{\text {out }_{3}}=T_{\text {in }_{2}}-\left(\Delta_{1}-\Delta_{2}\right) & \Delta_{2}=\frac{T_{2}}{6} \times \alpha \\
T_{\text {out }_{4}}=T_{\text {in }_{3}}-\left(\Delta_{2}-\Delta_{3}\right) & \Delta_{3}=\frac{T_{3}}{6} \times \alpha \\
T_{\text {out }_{n}}=T_{\text {in }_{n-1}}-\left(\Delta_{n-2}-\Delta_{n-1}\right) & \Delta_{n}=\frac{T_{n}}{6} \times \alpha
\end{array}
$$

Equation 4.3 allows the period of the generated output pulses to be calculated dynamically from the previous input pulse for a given $\alpha$. Figure 4.18 illustrates how this formula applies to the input and output pulses and how $\Delta$ changes with speed. This interpolation method was chosen because it offers a combination of computational efficiency whilst providing sufficient accuracy for the system. The timing error must be no more than 0.5 degrees from the target angle in order for the engine to operate smoothly. If the timing should fluctuate by more then 0.5 degrees, a noticeable change in performance can be seen through variations in cylinder pressure. 


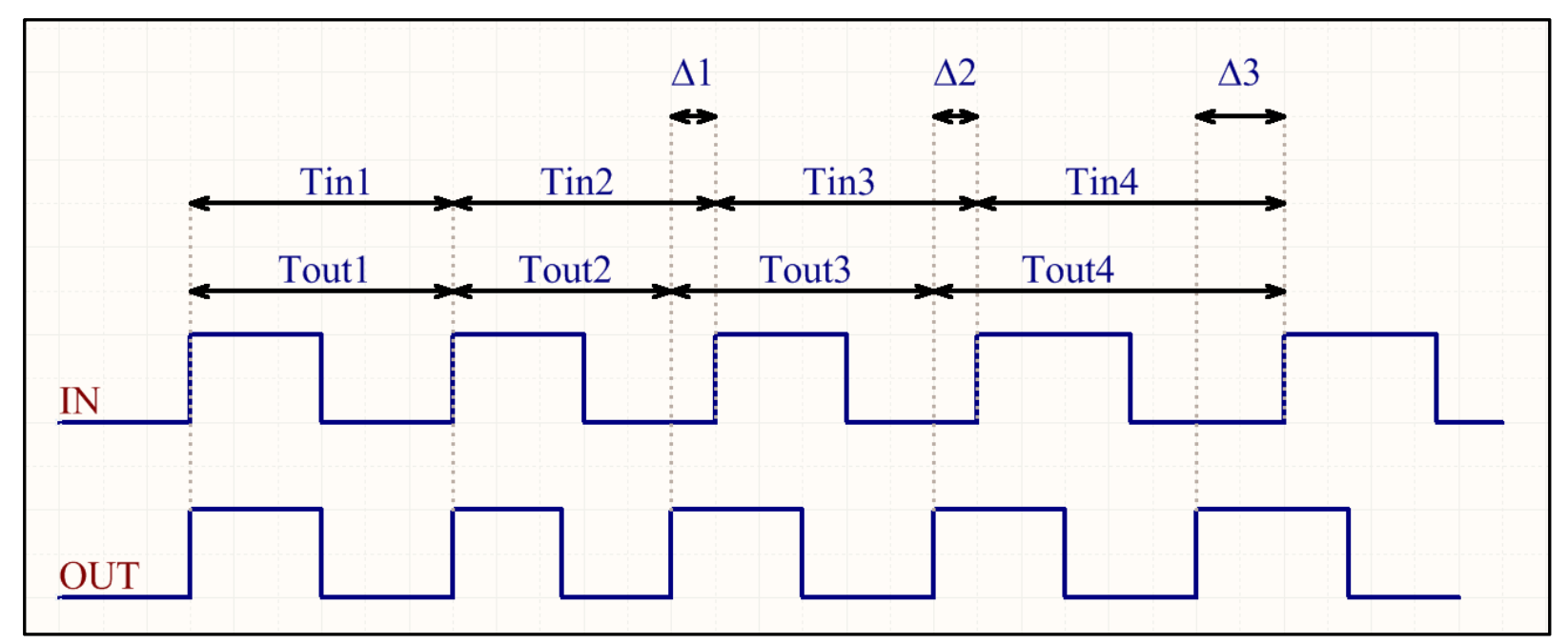

Figure 4.18 Timing advance diagram

This timing algorithm was implemented by the software designer in the final firmware, but was modified to use the information from a series of averages rather than just the previous two flywheel pulses. This is because, while the flywheel speed does vary considerably, the variation of adjacent pulses is quite small and the values of $\Delta$ will be similar. With such small variation in delta, continual calculation of the time shift is not necessary. Across a rotation of 30 degrees however, this variation will be greater and thus require an update of the shift in timing required.

To estimate the potential error of this algorithm, an engine speed of $2000 \mathrm{rpm}$ (33.3 revolutions per second) was taken as a typical engine speed. At this speed, the engine rotates at 1200 degrees per second with each 30 degree period of rotation taking $25 \mathrm{~ms}$. In $25 \mathrm{~ms}$ the engine is not likely to fluctuate by more than $\pm 2.5 \mathrm{rpm}$, which gives a maximum error of no more than $0.014 \mathrm{~ms}$. This error corresponds to a maximum error in timing advance angle of 0.017 degrees, much less than the maximum allowed error of 0.5 degrees.

Instead of $\mathrm{T}_{\text {in }}$ referring to 1 flywheel pulse, the DIM now counts 5 pulses (30 degrees). The same basic formula is applied but $\mathrm{T}_{\text {in }}$ is divided by $30^{\circ}$ before being multiplied by $\alpha$ when calculating $\Delta$. By performing the calculations every 30 degrees instead of every 6 , the computational power required is reduced by a factor of five. The timing advance signal also anticipates the two missing pulses used as a reference point by the OEM ECU and produces the same gap for this special case. 


\subsection{Summary}

With the hardware built and software tested, the prototype DIM is complete. Because the DIM forms only one half of the dual fuel system, it must now be tested in conjunction with the dual fuel controller, which send commands to the DIM during dual fuel operation. The initial testing and configuration of the dual fuel system and test environment is described in chapter 5. 


\section{$5 \quad$ System Testing}

This chapter describes the systems used for testing the various components of the dual fuel system, from the equipment used to configuring the test cell, engine, and various data collection methods.

\subsection{Equipment Used}

To measure the performance of the dual fuel system on the $4 \mathrm{HK}$ engine, the engine had to be run at various speeds, simulating a load placed on the engine similar to that of a truck as it travels along a road (going up and down hills, etc.). This allows us to test the dual fuel system across the full range of loads an engine is likely to experience. While the engine is running, several internal and external parameters are measured to monitor the engine output and performance. This allows us to determine the effect the dual fuel system has on the engine itself, and to keep the engine within a normal operating range. The key pieces of equipment used for this testing are described below.

\subsubsection{Dynamometer}

The dynamometer is used to measure the power output of the engine. It does this by measuring the torque applied to the rotating drive shaft. The dynamometer can also place a load on the engine, allowing us to see how the engine performs under varying driving conditions.

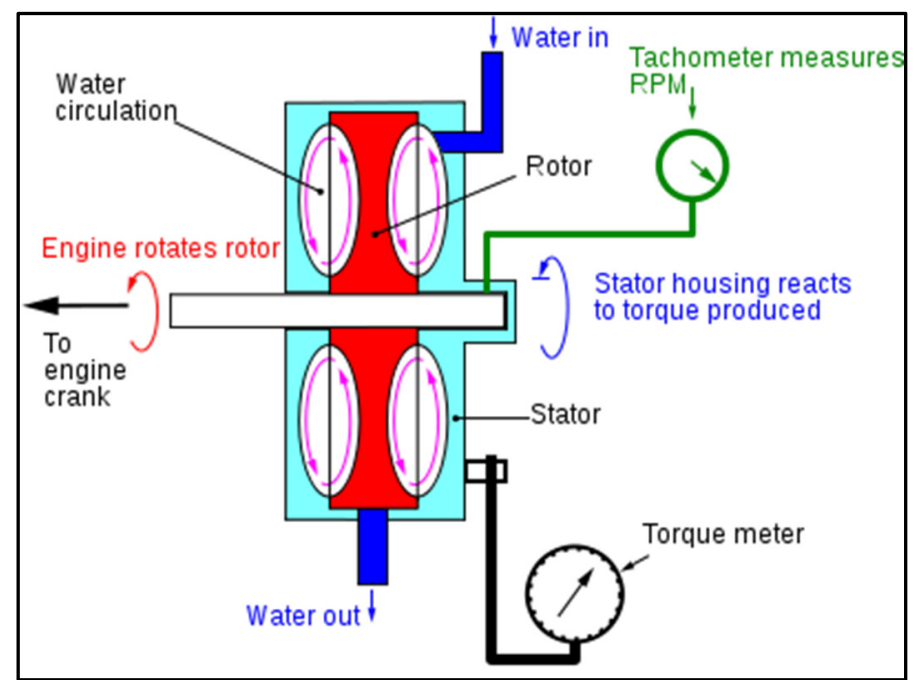

Figure 5.1 Diagram of water-brake dynamometer [35] 
The dynamometer used is a water-brake type dynamometer. This type of dynamometer consists of a rotor driven by the engine inside a stator housing, which is filled with water (see figure 5.1). Water flows through the housing at a given pressure and sluice gates control the flow. The more water that flows through the housing, the more opposing force is applied to the rotor. This causes the housing to attempt to rotate in the opposite direction and this rotation is measured using a strain gauge, the output of which is proportional to the torque applied to the engine.

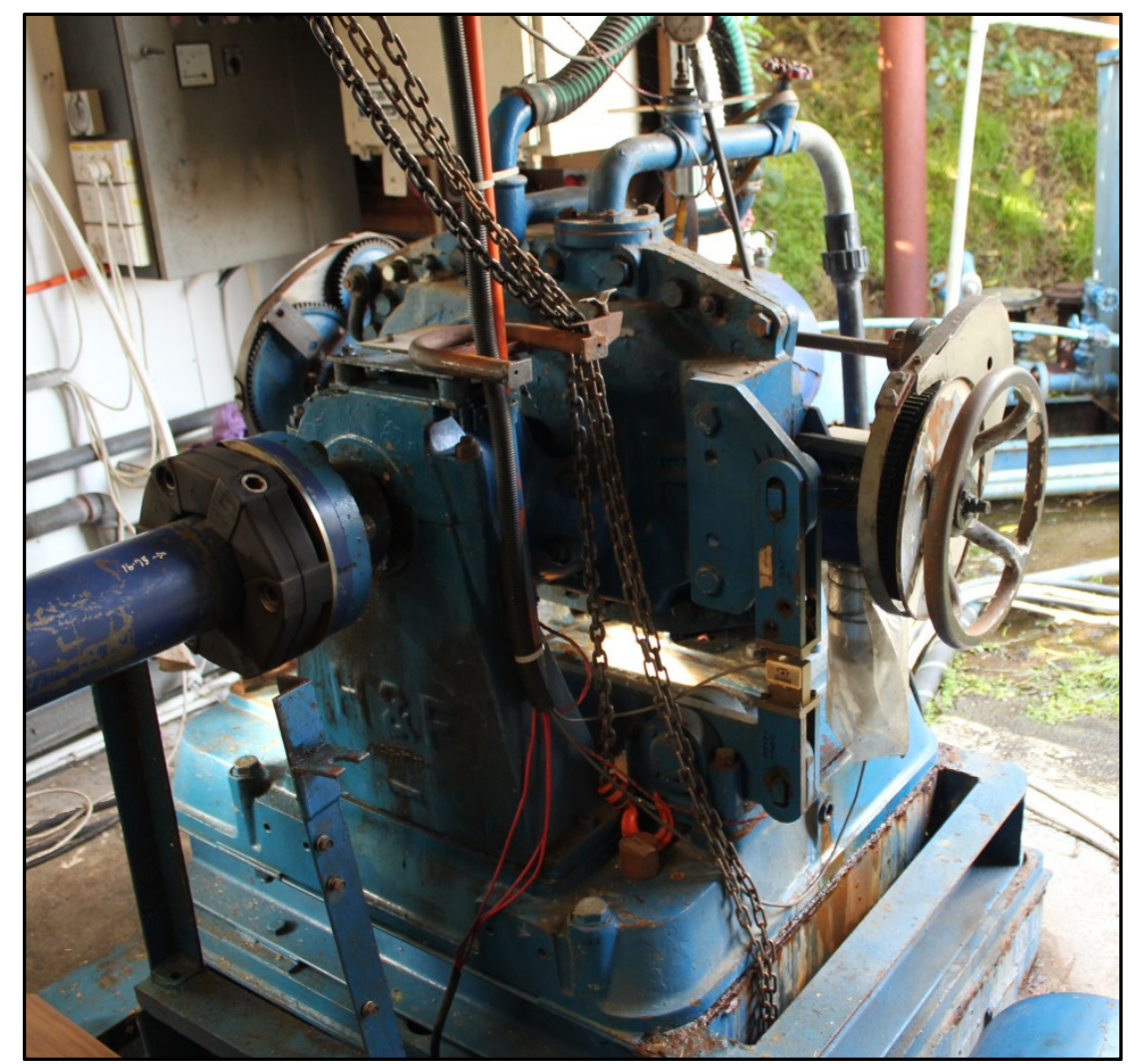

Figure 5.2 DieselGas dynamometer

The DieselGas dynamometer (figure 5.2) can be controlled via a PC to hold either a specific speed or a specific torque. When the dynamometer is holding speed, it will vary the torque until the desired speed is reached. Increasing the speed of the engine will cause the dynamometer to increase the load. If the desired speed is higher than that of the engine, the dynamometer will not apply any torque to the engine. When holding a specific torque, the dynamometer will move the sluice gates until the required torque is reached and maintain it, regardless of engine speed.

The sluice gates on the dynamometer are moved by a proportional-integral-derivative (PID) controller which receives feedback from the speed sensor and the strain gauge. The desired 
torque/speed is selected via a PC in the control room and the PID controller actuates the sluice gates to maintain the selected parameters.

The sluice gates can also be manually set to a specific position, which will place a particular load on the engine. There is no feedback with this mode however, so it is not normally the preferred way of operating the dynamometer. During the testing of this engine, this proved to be the easiest way to perform some load tests due to the particular governor characteristics of this engine, as discussed in section 5.3.1.

As well as taking input from a PC, the dynamometer outputs the current RPM of the driveshaft and also the load applied. From the measurement of the strain gauge the torque and power of the engine can be calculated. These values are displayed and logged through the LOGINT program (discussed in detail in section 5.1.3).

\subsubsection{CONAN}

CONAN is the name of the proprietary DieselGas data logger used to collect data from the system. It takes input from a number of sensors around the test cell, including:

- Intake manifold air temperature (IMAT)

- Intake manifold air pressure (IMAP)

- Diesel temperature

- Common rail pressure

- Air flow

- Diesel flow

- Gas flow

- Exhaust temperature

- Exhaust oxygen level

- Exhaust hydrocarbon level

CONAN has 40 channels through which to record data. These channels can record analogue voltages, or count pulses. Channels are configured via a serial connection by specifying the output channel number, channel type, input channel number and sensitivity. An example configuration command would be: 
This instruction is a channel select ("CS") instruction which links output channel 14 to hardware input channel 14, which is configured as a 14-bit analogue ("a") input channel. The range is set to " 3 ", which the CONAN datasheet specifies as a $5 \mathrm{~V}$ input range with a $305 \mu \mathrm{V}$ resolution (14-bit ADC). The range can be set to either 24, 12, or $5 \mathrm{~V}$, with an argument of 1 , 2 , or 3 rspectively.

CONAN records these raw values and sends them to the LOGINT program to be displayed and recorded on a PC. The data is sent as a text string over a serial connection. CONAN is the primary method of data collection in the test cell to monitor the performance of the engine. Because CONAN only logs raw data and sends this to a PC via serial communication, no data analysis is performed by CONAN itself.

\subsubsection{LOGINT}

LOGINT is a program written by DieselGas International which collates data from CONAN, the DFC, DIM, and the dynamometer and displays it on-screen. As well as displaying all the information from these sources in a way that is easy for an operator to observe visually (figure 5.3), it also allows the operator to record these values into a log file (see appendix 3) for later analysis.

LOGINT takes raw data from CONAN and converts it to useful information through a DAT file, which contains the mathematical functions required to calculate and derive the actual sensor values, such temperatures and pressures, from the digital outputs of CONAN. For example, an analogue channel from CONAN may have a digital output between 0 and 16384 . In order to display the temperature this output represents, it may have to be multiplied and offset by a particular value. The DAT file contains these transfer functions. An example of the DAT file structure is shown below. The full DAT file used can be found in appendix 4 .

$$
\text { Diesel In [Diesel_In_c }=(\mathrm{c} 14-874) / 34.93,1,29,42] \varnothing \mathrm{C}
$$

The above code snippet shows how a variable for displaying diesel temperature is created and displayed on-screen from the raw CONAN variable, and given upper and lower bounds. "Diesel In" describes what label is shown on-screen. Inside the brackets, a variable called "Diesel_IN_C" is assigned a value derived from the CONAN data channel C14 and the transfer function relating to that sensor. 
The transfer function for this particular sensor was derived by a previous engineer and is given as:

$$
\text { Diesel temperature }=\frac{(\text { CONAN channel 14) }-874}{34.93} \quad \text { (Equation 5.1) }
$$

The variable is then rounded to 1 decimal point and given upper and lower limits of 42 and 29 degrees respectively. After the closing bracket, the unit of ${ }^{\circ} \mathrm{C}$ is written on-screen. When the value of the variable exceeds the upper or lower limits the variable is highlighted red, to alert the operator to a fault condition.

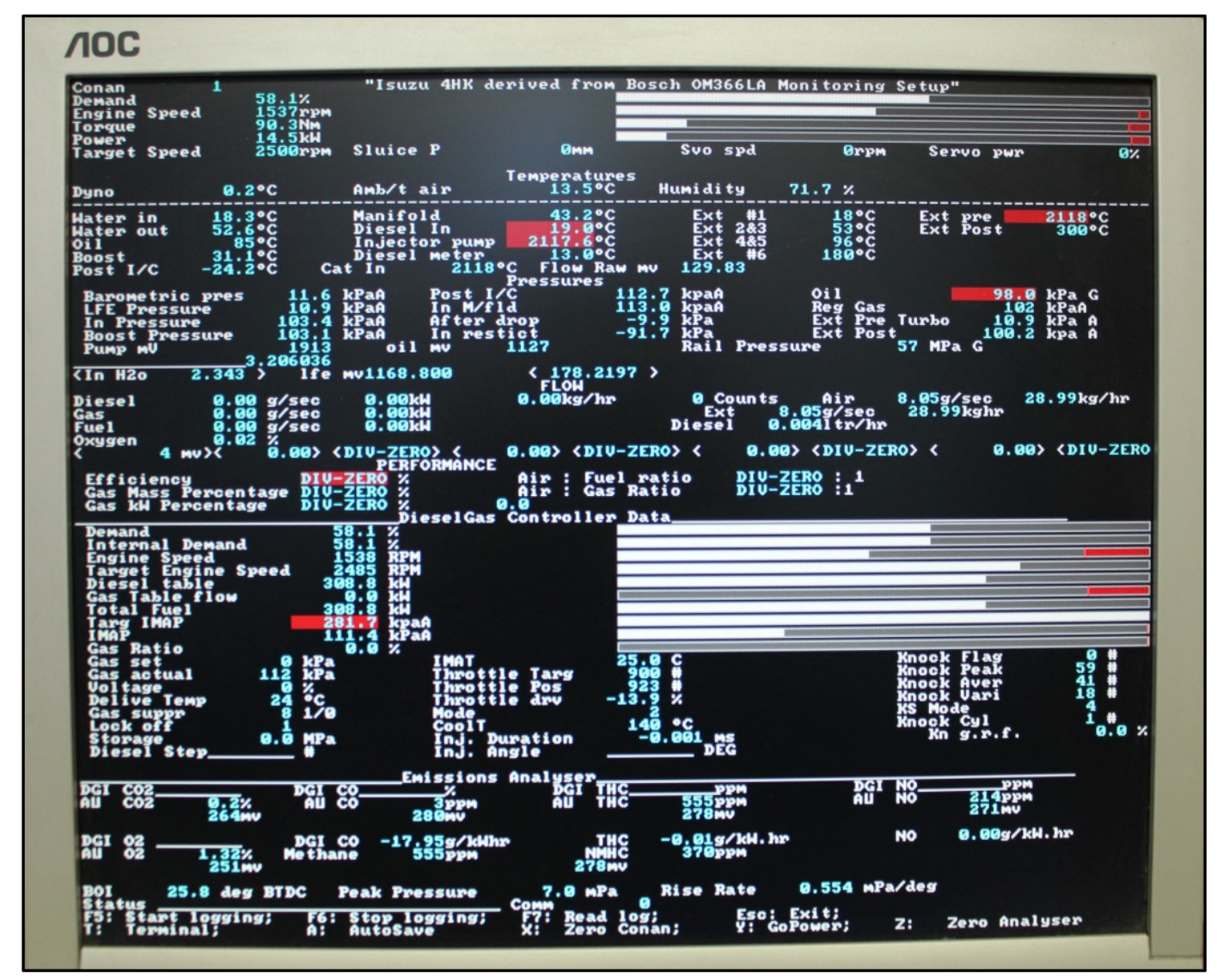

Figure 5.3 LOGINT screen

While CONAN was already configured for most sensors used in the test cell, one of the additional values which requires recording is the common rail pressure sensor. From the manufacturer's data sheet, it was found that this particular sensor has the output characteristics shown in figure 5.4. 

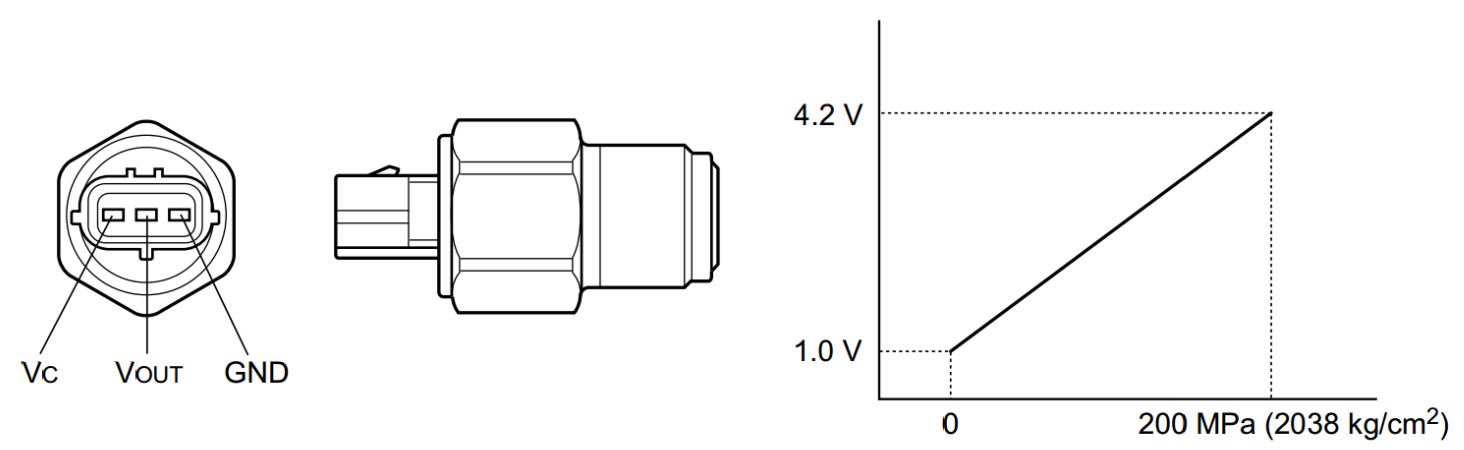

Figure 5.4 Rail pressure sensor and output characteristics [36]

At $0 \mathrm{MPa}$ the sensor outputs $1 \mathrm{~V}$, and at $200 \mathrm{MPa}$ the output is 4.2 volts. For a 14-bit analogue to digital conversion, this gives a resolution of $305 \mu \mathrm{V}$ per increment. Therefore, to determine the pressure from the output of CONAN, the transfer function will be:

$$
\text { Pressure }=\frac{\frac{\text { ADCreading }}{16384}-3276.8}{52.43}=\frac{\text { ADC Reading }}{312.5}-62.5
$$

(Equation 5.2)

LOGINT reads the DAT file on start up, and as it is receives data from CONAN, the DFC, dynamometer, and DIM, it calculates the derived values and displays them on screen. LOGINT also has a terminal facility to allow an operator to set parameters within the dynamometer, DIM, and DFC.

\subsubsection{DGTech}

DGTech is a proprietary application developed by DieselGas International to communicate with the original DGCs. For this project it is used to talk to the DFC, which controls the DIM. The DFC operates as a standard DieselGas Controller, aside from the fact that it uses the DIM to both provide timing information, (instead of a timing wheel mounted on the engine), and to control diesel delivery, (instead of a stepper motor).

DGTech serves two main purposes: to configure the DFC and to log the DFC performance. For the DFC to function correctly, the calibration and fuel tables must be configured and uploaded beforehand. The fuelling table determines (from demand and speed) how much 
diesel and gas to inject. Because this dual fuel system is designed to dynamically react to changes in speed and demand, the fuel table must be comprehensive enough to cover all expected loads and speeds. Through running the engine and manually fine-tuning the relative mixtures of diesel and gas, it is possible to generate data points to form the basis of the fuel table. DGTech can then take these experimentally determined data points and interpolate intermediate values to approximate the required fuel delivery at all loads.

DGTech can also monitor and log DFC data in real time, providing a graphical representation of how the system reacts to loading. Variables which can be logged include the position of the air throttle and gas valve, as well as the input demand, timing advance, and injection window parameters. DGTech communicates to the DFC via a serial connection and can control DFC variables in real time as well as changing which mode the DFC is in (e.g. dual fuel or diesel mode, or testing/manual control modes).

\subsubsection{PSCOPE}

PSCOPE is a program used to display the cylinder pressure over a full combustion cycle. The pressure transducer installed in the glow plug port on cylinder \#1 (as described in chapter 4.3.1), drives a charge multiplier which feeds an output voltage to a fast Analogue-to-DigitalConverter (ADC) in a PC running PSCOPE. PSCOPE uses an external timing wheel mounted on the front of the engine as a reference signal, which allows the program to display the pressure trace in relation to TDC as shown in figure 5.5.

The shape of the pressure trace provides a graphical representation of the combustion of fuel inside the cylinder and can be used to see if the engine is running close to knock conditions. By reducing sharp peaks in cylinder pressure, smoother combustion and quieter running can be achieved, as well as reducing the likelihood of knock occurring. Due to the high cost of precision pressure transducers, only cylinder \#1 is monitored. The other cylinders are assumed to have similar performance and any difference in cylinder pressure is likely to be due to inadequate mixing of the air and gas from the injection process. If knock occurs in any of the other cylinders during operation, the DFC will be able to detect this using the installed knock sensor. By using the timing information and the known firing order of the cylinders, the DFC can infer which cylinder is knocking and produce a log of the event. 


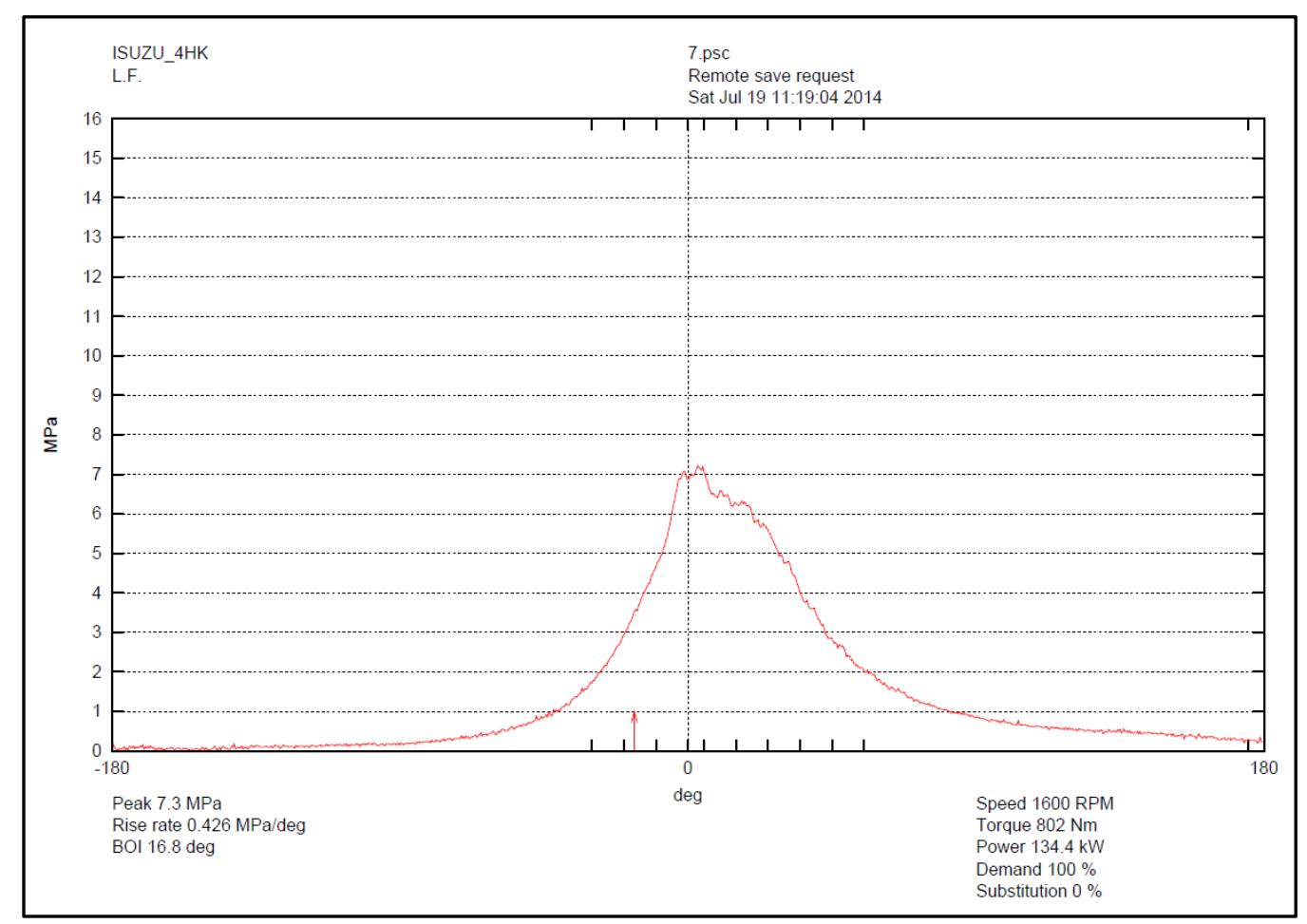

Figure 5.5 Example PSCOPE pressure trace

\subsection{Test Environment}

The Isuzu 4HK engine was mounted on a dynamometer in a test cell previously used by DieselGas International for development of their mechanical dual fuel kits (figure 5.6). The test cell contains the various facilities required to run an engine such as air, fuel, and water as well as the electronic hardware required to control engine variables and monitor performance. The test cell contains a control room from which the engine can be controlled, as well as the dynamometer and other external variables.

The test cell allows full control over external variables such as air, fuel, and water temperatures in order to maintain consistency in the experiments. Before any engine testing could begin, the test cell had to be configured for the 4HK1 engine. Air, water, fuel, and exhaust facilities had to be connected to the engine, as well as the mounting of additional sensors such as knock, intake manifold air pressure and temperature (IMAP and IMAT), and cylinder pressure sensors. An air throttle and gas valve were also mounted in the air intake just before the inlet manifold at this stage in preparation for dual fuel operation. 


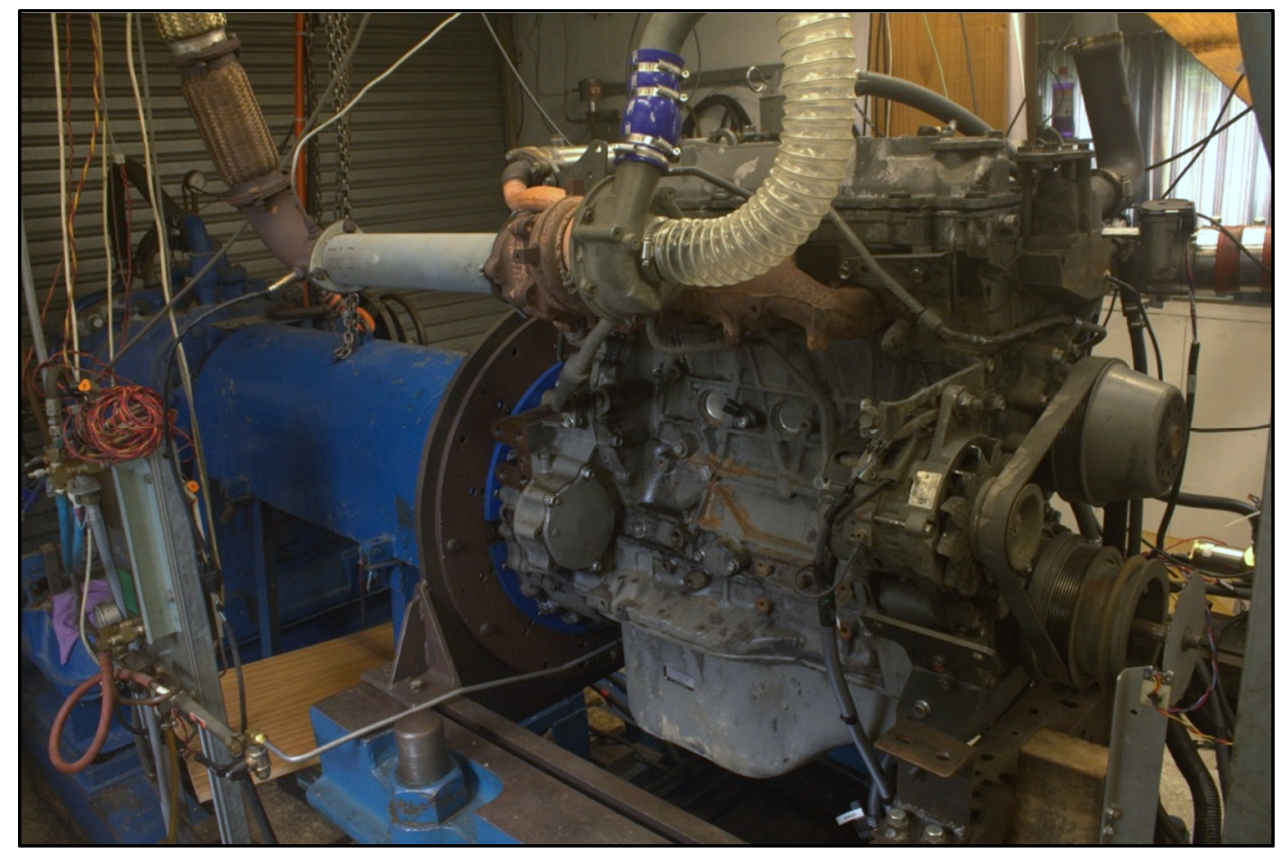

Figure 5.6 Isuzu 4HK1 engine on the test bed

\subsubsection{Configuring the Test Cell}

Because there are so many variables which can affect the operation of the engine, it is necessary to keep as many of these variables as constant as possible. To make sure any variance in engine performance is only due to dual fuel operation, the air, water, and diesel temperatures are kept constant throughout the experiments, as is gas delivery pressure.

Both air and diesel change in density proportional to changes in temperature. In order to keep the density constant, the air and fuel input temperatures are both controlled by heat exchangers. The heat exchangers can increase and decrease the temperature of the intake air and diesel through the use of flowing hot and cold water. The flow of hot and cold water is controlled by a temperature controller which is configured from within the control room of the test cell. The system can be set to maintain a specific temperature which allows the tests to be performed with consistent air and fuel temperatures, thus removing error in the air/fuel ratio.

Inside the control room are the computers running CONAN, LOGINT, DGTech, and PSCOPE. From the control room, an operator can monitor and manipulate all variables and accessories attached to the engine. There is also an emergency cut-off for all fuel, gas, and electrical services as a safety measure. The control room computers allow configuration of 
the DFC, DIM, dynamometer, and various other system variables, while monitoring the performance of the engine through LOGINT and DGTech.

\subsubsection{Configuring the Engine}

With the engine mounted on the dynamometer, the electrical connections between the engine and OEM ECU had to be made. In order to determine how the OEM ECU is connected to the various engine sensors and actuators, schematics for the engine and the hydraulic excavator from which it came were provided under licence from Cable Price Ltd. Because the excavator in which this engine runs has many other auxiliary functions, the wiring loom has many connections which are surplus to requirements. It was therefore necessary to thoroughly understand these schematics in order to identify the connectors and wiring required by the engine to operate independently.

In a hydraulic excavator, this 4HK1 OEM ECU is driven via a CAN bus. The excavator has its own controller unit which takes information from the operator controls and various other sensors, and calculates a target demand for the engine. This controller then tells the engine ECU what the demand is via the CAN bus. The machine is designed this way to improve fuel efficiency and power delivery across the various modes of operation of the machine.

Because it was impossible to obtain a machine controller to drive the engine ECU, an alternative method of supplying a demand signal had to be found. Through careful analysis of the engine manuals, it was discovered that in the event of a CAN bus failure, the ECU should be able to revert back to a simple analogue voltage signal to determine demand. While this feature was not documented in the excavator workshop manual, there were two connectors (hidden behind the armrest in the cab of the excavator) described in the wiring loom schematic which would connect the demand control knob in the cab directly to the engine ECU, bypassing the machine controller.

To determine the validity of this theory, it was tested in an excavator using the same model engine. By unplugging the CAN connection to the machine controller, and plugging the demand knob directly into the engine ECU, we found that we could control the engine demand directly without the need for CAN communication. This now meant we could control the engine in the test cell with a simple analogue voltage. 
To provide this analogue voltage to the ECU, a potentiometer was wired up to deliver a 0-5 V signal. The demand of the engine is proportional to the voltage on the respective ECU pin. Increasing the voltage increases the demand (and therefore speed) of the engine. The demand signal was also fed to the DFC which requires a demand value for the calculation of fuel delivery.

Once the OEM ECU was connected to the engine, the starter and emergency cut-off circuits were connected. With these circuits connected, turning the key inside the test cell control room would crank the engine. Initially the engine did not start because of a wiring fault in the emergency cut-off system, as the emergency cut-off switch was pulling the ECU pin voltage up and not down. Once this problem was resolved the engine began to run, but would run rough, produce a lot of smoke, and surge. It was thought that there may have been an issue with sticking valves, so the rocker cover of the engine was removed to inspect the mechanical components of the valve train. These all seemed fine, so the injectors were removed for inspection.

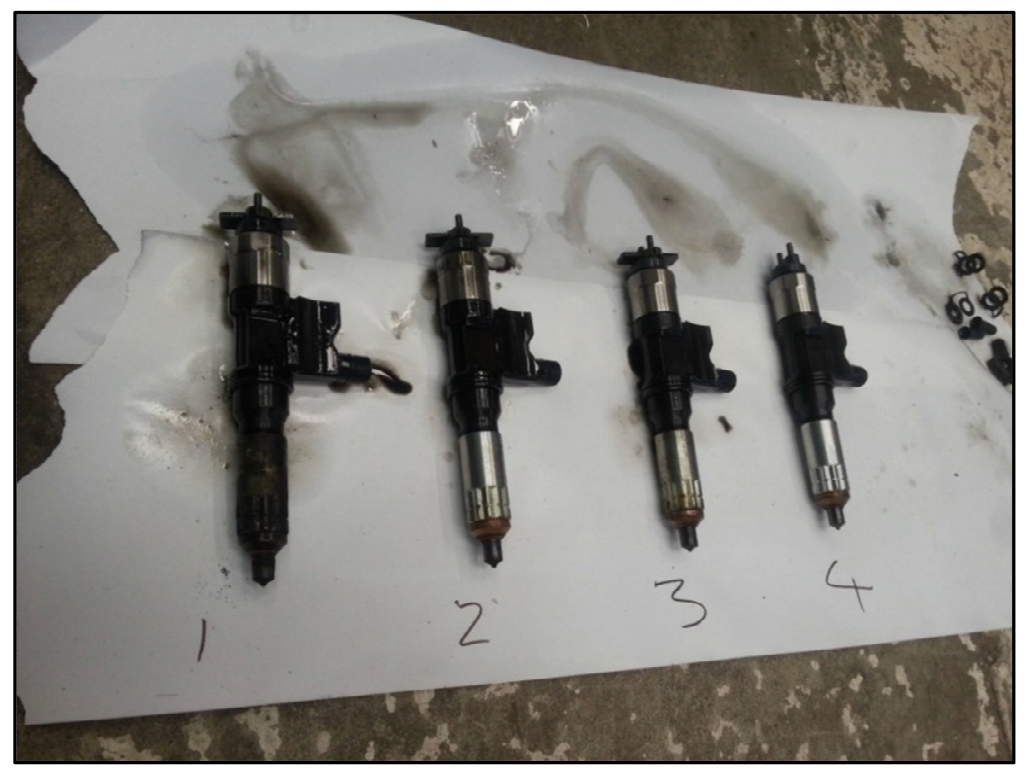

Figure 5.7 Injectors removed from engine. Note carbon build-up on \#1

It was found that the injector for cylinder number 1 was fouled and was likely the cause of the rough running of the engine (Figure 5.7). All four injectors were replaced, and the OEM ECU updated with the new injector calibration figures. The OEM ECU update was performed by CablePrice Ltd., as they own the proprietary hardware and software to perform this task. With the new injectors installed, the engine ran smoothly and did not produce smoke. The next step was to interrupt the timing signals coming from the engine. 
The wiring to the cam and flywheel position sensors was cut and connectors crimped on the ends. This allows the signals to either be routed through the DIM or passed straight through to the OEM ECU. During the testing phases only one sensor would be routed through the DIM at a time, and the others plugged straight into the OEM ECU. This allowed isolation of each signal and thus helped identify any errors occurring with the signal manipulation.

The injector leads were cut in a similar fashion and connectors crimped onto the ends so they too could be sent through or bypass the DIM as required. Being able to bypass the dual fuel system and plug the sensors and injectors directly back into the OEM ECU ensures the engine can always be run as originally designed on diesel by simply unplugging a few connectors. This is an important feature of the dual fuel system providing safety and reliability, should the dual fuel system fail.

\subsubsection{Configuring the Dual Fuel System}

For dual fuel operation, an air throttle and gas injection valve unit were installed upstream of the inlet manifold, as seen in figure 5.8. These are the only mechanical devices required for the dual fuel conversion process and are typically installed in the hose leading from the turbocharger/intercooler to the intake manifold. This unit (designed by DieselGas) controls the air flow into the manifold and also injects gas. Both of these valves are controlled via the DFC.

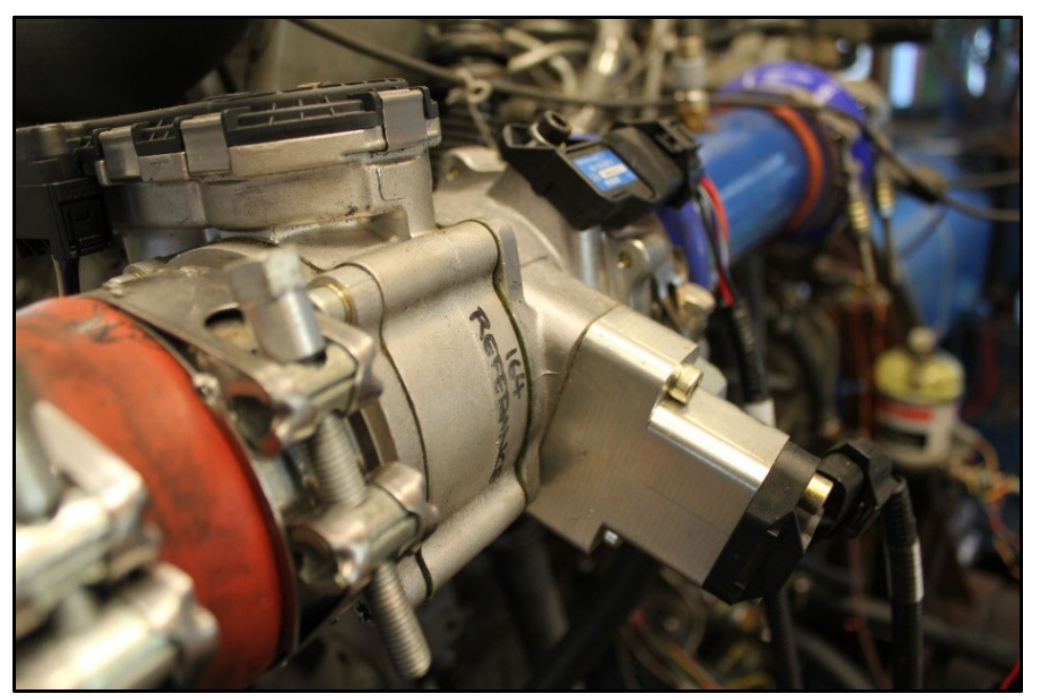

Figure 5.8 Throttle body and gas valve installed in air intake

Once the engine and test cell were configured, the DIM and DFC were mounted next to the engine (Figure 5.9) and plugged in. The appropriate connections between the two were made 
and communications between the new hardware and the control room were established. The dual fuel system was now ready to configure.

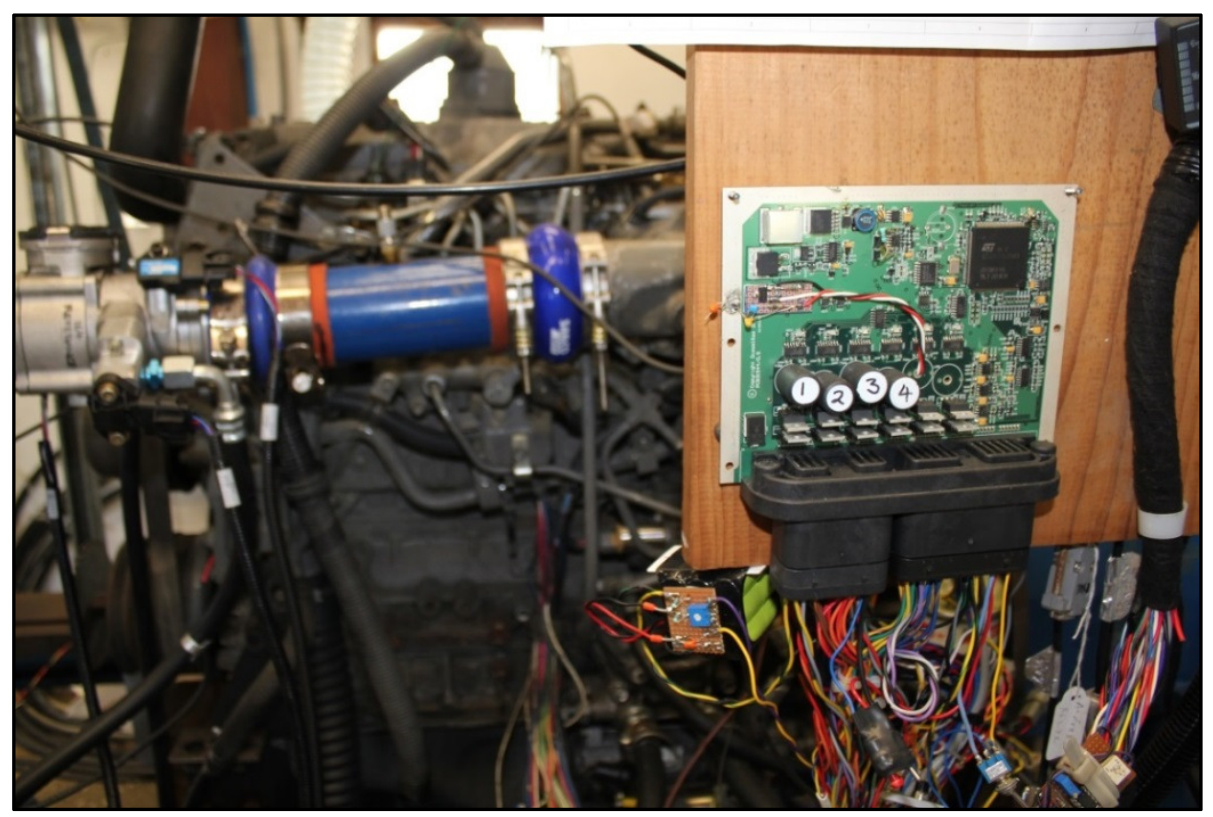

Figure 5.9 DIM mounted next to engine

The DIM has three main variables to control: the angle before TDC that the injection window opens; the duration of the injection window; and the phase shift of the timing signals sent to the OEM ECU. The variables which hold these values are:

- inj.window

- inj.dur

- inj.timing

In dual fuel mode, the values of these parameters will be sent to the DIM by the DFC. These parameters affect the operation of the DIM in the following ways:

- To allow an injector pulse through to the engine, the DIM will open the injection window $x$ degrees before TDC, where $x$ is the value of inj.window.

- With the injection window open, the DIM starts monitoring the current transducer. When current flow is detected, the DIM starts counting and closes the window $y \mu \mathrm{s}$ after current starts flowing, as defined by the value of in $j$. dur.

- The value of inj.timing sets the angle $\alpha$ by which the timing signals from the engine are advanced and sent to the OEM ECU. 
During the course of operation, the angle of injection and the injection duration variables must change dynamically in response to speed and load. Therefore the DIM must adapt to these changes and alter the inj.window and inj.dur values according to the instructions sent by the DFC. In practice, the inj.window and inj.dur values will be stored in a lookup table in the DFC, which sends these values to the DIM. This is effectively the same as the fuelling tables previously used in the original DieselGas Controllers which would control the position of a stepper motor. For initial testing, the DFC fuelling tables were not used and the variable values were set manually by assigning them via a terminal program. Once the variables were observed to work correctly under manual control, it was possible to control the four main system variables simultaneously by using a device called a pots box.

The pots box (figure 5.10) is an external controller with 4 dials to control 4 different system variables within the DFC. These are set up to control the diesel, air, gas, and timing advance of the dual fuel system. The diesel knob increases/decreases the injection duration (inj.dur) variable in steps of $1 \mu \mathrm{s}$ and the timing knob shifts the phase of the timing signals, advancing/retarding the timing of the engine by 0.1 degree increments. The air and gas knobs open and close the throttle body and gas valve respectively.

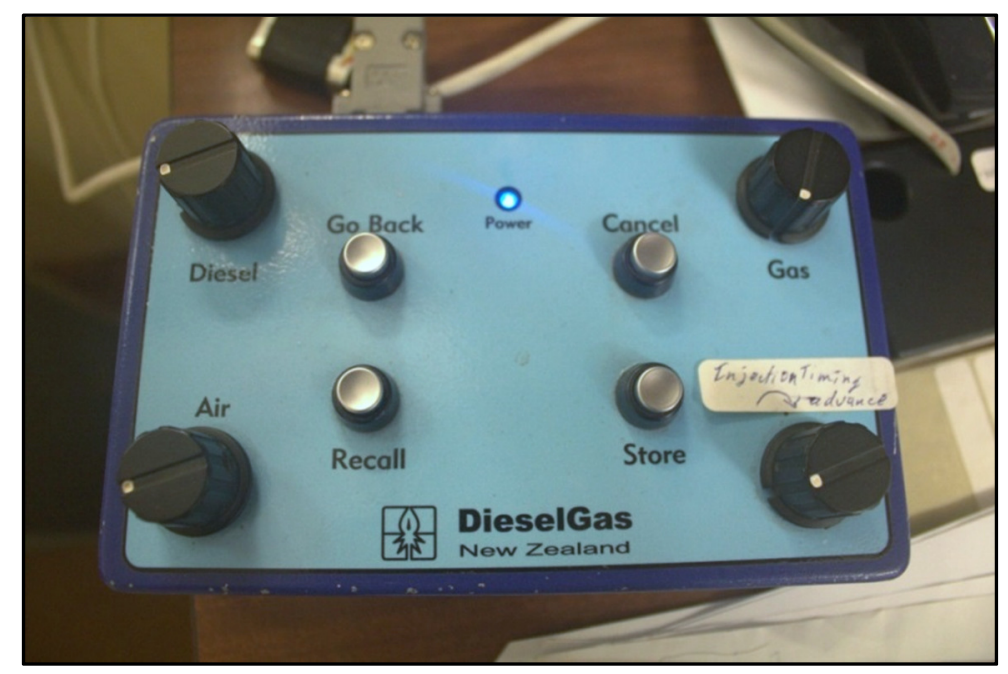

Figure 5.10 Pots box control unit

In initial testing, the pots box was used to control the system variables manually. That is, the DFC would not use any of its internal control systems to maintain engine operation. This initial test was performed to observe how changing the injection duration and timing affects the behaviour of the engine. 
After testing using the pots box in direct control mode, the DFC was placed in sensor mode, which takes input not only from the pots box but also monitors its own sensors to ensure the engine keeps running. If one of the variables becomes limited by another variable (i.e. reducing the diesel so far that the engine will stall), the dial will have no effect.

The pots box is used in this way to control the target variables, and not the direct output variables. A target diesel flow is set using the pots box, and the system will try to reduce the injector on-time to achieve that flow. If it cannot reach this level, it gets as close as it can but not so far as to stall the engine. With the engine running in pots sensor mode, the engine was ready to begin trials.

\subsection{Data collection}

This section describes the methods by which the data describing engine performance and dual fuel system operation was obtained, firstly on the BT-50 and then the 4HK1 engines.

\subsubsection{BT-50 Data Logging}

In order to visualise the operation of the BT-50 utility vehicle while under load, a National Instruments datalogger was used to capture various engine signals while driving. The timing signals, injector pulses and common rail pressure were recorded, along with derived values for RPM, cylinder pressure and injector on-time (Figure 5.11).

The data is captured on a PC running custom LabVIEW software designed by DieselGas International which logs the raw data and processes it for post analysis. This program allows one to observe the performance of the engine and ECU under load while driving. In order to collect meaningful data, the engine was run at various speeds and demands.

The analysed data showed how the injectors are driven by the ECU, both in terms of the amount of fuel injected (inferred from the injector on-time), but also the point at which the fuel is injected in terms of the angle of engine rotation relative to TDC.

This information provided insight into the operation of the BT-50 ECU, but because the actual load on the engine was not known, it is not possible to calculate exact values for engine power output and efficiency through this method. The data collected was used as a starting point for the initial concept and development stages and was not used past the first prototyping stage. 


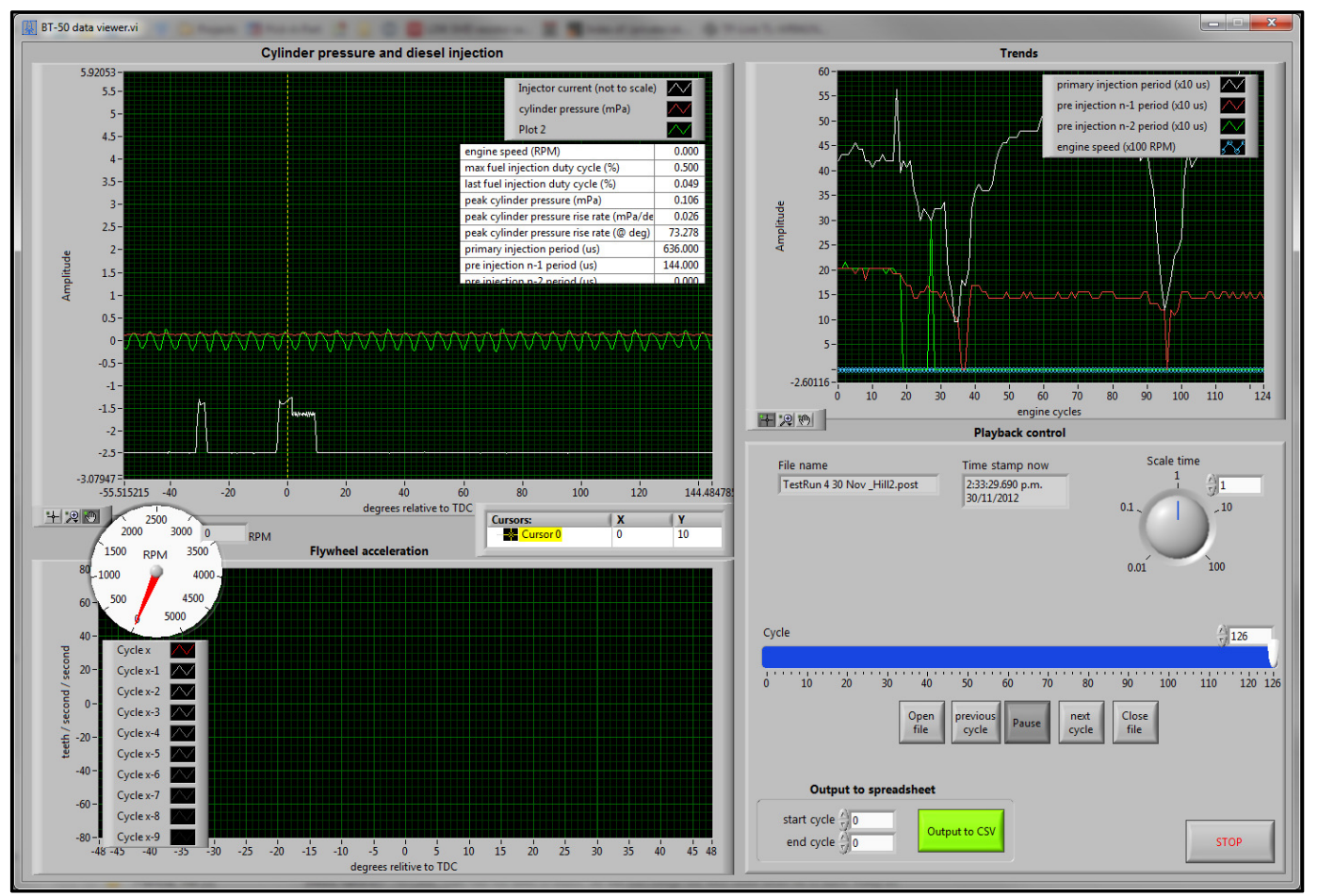

Figure 5.11 LabVIEW datalogger interface

\subsubsection{HK1 Characterisation}

Before the performance of the dual fuel system was tested on the $4 \mathrm{HK}$ engine, it was first characterised running on diesel only. This would serve as a baseline reading against which the performance of the dual fuel system would be compared. Once the engine had been characterised running solely on diesel, the dual fuel system was plugged in and run.

Before the dual fuel tests could be performed however, the DFC had to be configured in DGTech. The fuelling tables had to be updated with the new time-based values which correspond to the injector opening times. The upper limit of the injector on-time was originally based on the total injector on-time as measured on an oscilloscope from the engine itself when running at full load. This value will be the longest injector pulse the OEM ECU will ever produce, so the DIM should not need to keep the injector window open for any longer than this length of time. The lower limit of the fuelling table was set by running the engine at no load, and reducing the injection pulse until the engine stalled. While running on dual fuel the injection pulse could technically be shorter, but this value was chosen as a safe starting point. 


\subsubsection{Tests Performed}

To measure engine performance, two main tests are performed: a lug test and a droop test. The lug test is a measure of the absolute maximum power the engine can provide. The engine is set to the $100 \%$ demand and load is applied until the maximum power output is achieved, then increased further until the engine is about to stall. This gives a graph of torque vs speed (Figure 5.12), which outlines the maximum torque the engine can apply at $100 \%$ demand. As this is the maximum power output of the engine, all subsequent test points will lie beneath this curve. The best possible scenario is that while running on dual fuel, the power output of the engine can be matched, or at least get close to the maximum, but it is not expected to exceed the power output from running solely on diesel.

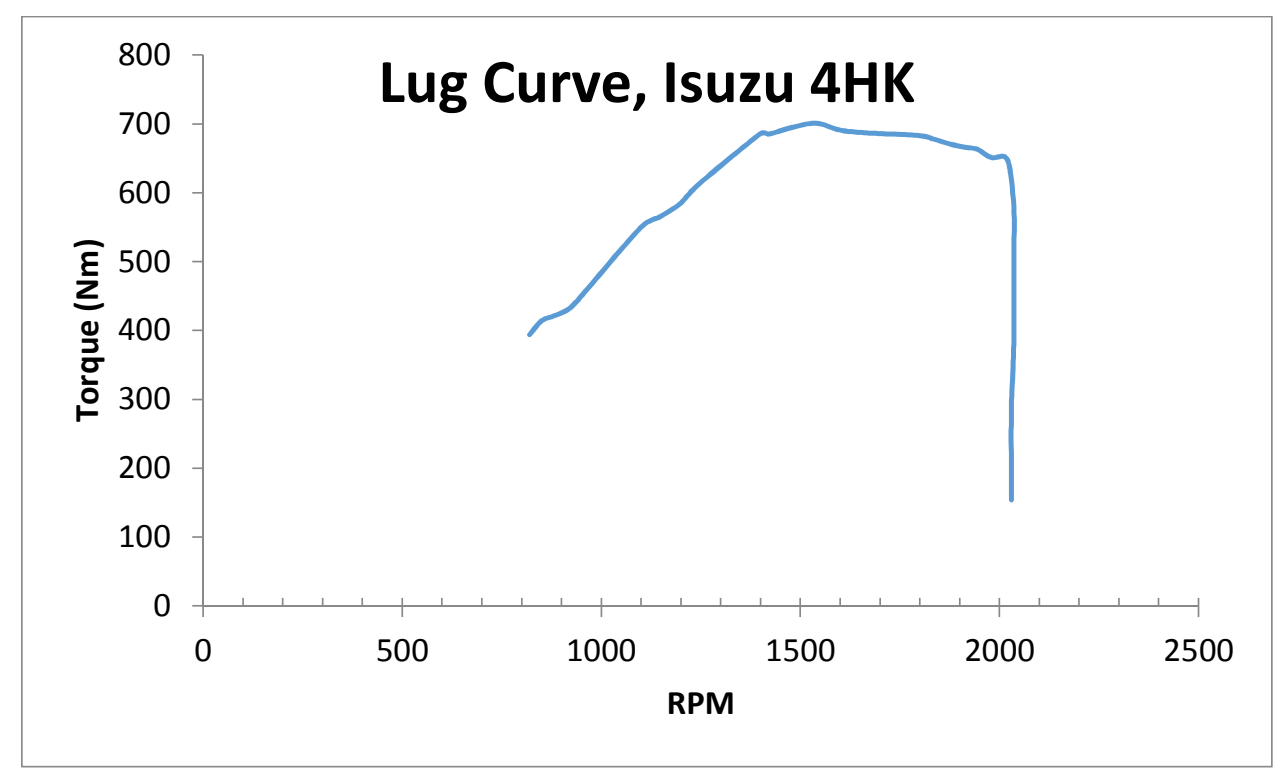

Figure 5.12 Example lug curve taken from 4HK engine

The next test is a droop test. This test provides an indication of the performance of the governor of the engine and its response to load. The engine is set to a given demand (speed) and torque applied until the engine can no longer hold this speed and begins to slow down. Figure 5.13 shows the family of curves which describe how the engine responds to increases in load at a given demand and how effective it is at maintaining that speed. For an engine with mechanically-driven injectors, the slope of this graph is often shallow, as the engine struggles to maintain the exact initial RPM, as the governor is a mechanical device with mechanical losses. However, in common rail engines the injectors are governed electronically by software, so a constant RPM is easier to maintain through the use of control systems. Therefore, the slope of the droop curves should be practically vertical at the drop-off point, as the governor compensates for the load placed on the engine. 


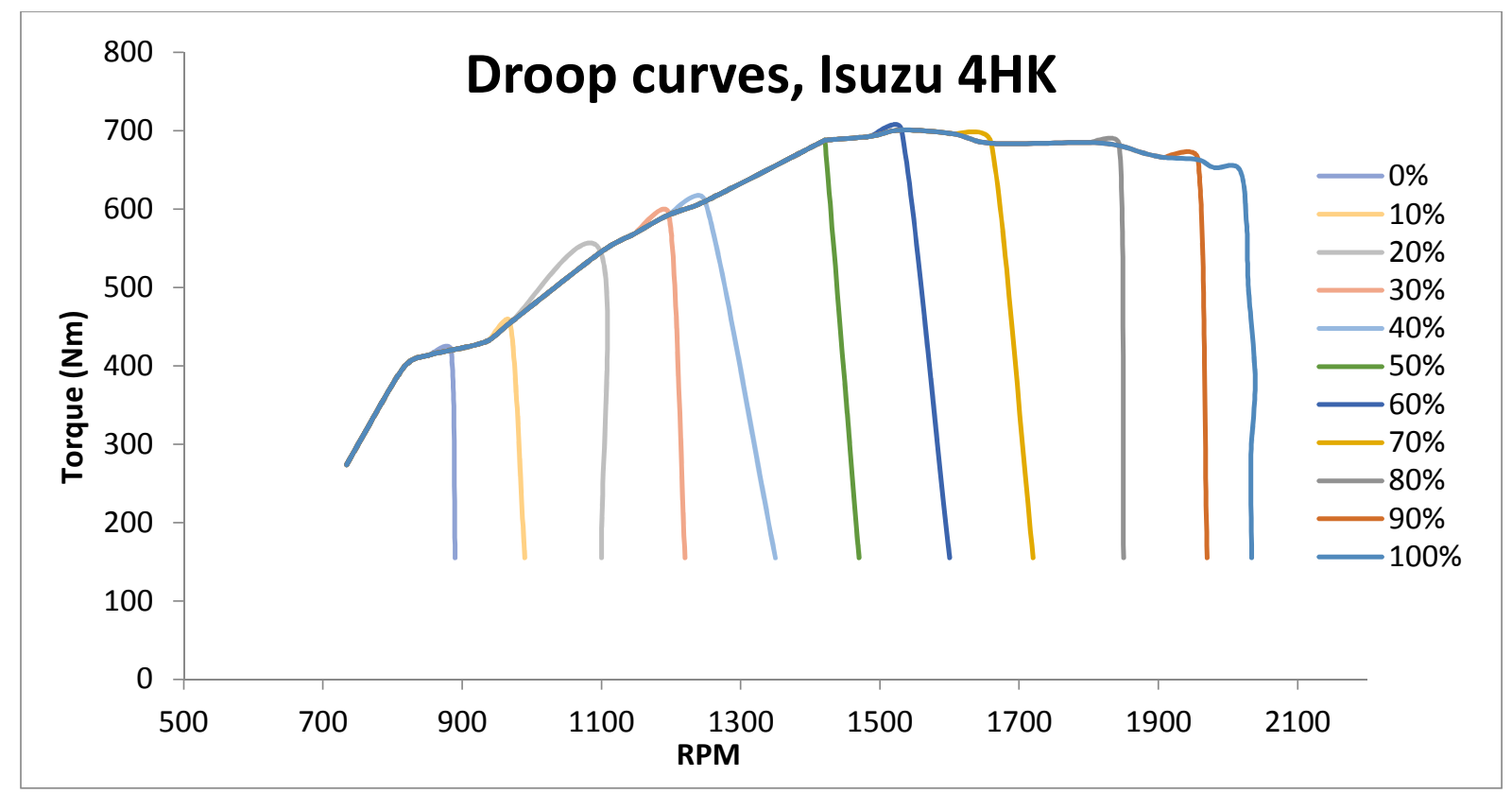

Figure 5.13 Droop curves of Isuzu 4HK engine

Having data points for the power output of the engine at every demand setting, and at every load, produces a map of how the engine should perform under every condition found on the road. However, these maps will change with fluctuations in ambient air temperature and pressure, and also as the engine warms up and cools down. For the purposes of this project, the engine will be tested under one standard air and water temperature.

The testing of the dual fuel system was performed in stages. First, the injection windowing was tested to ensure the injection pulses can be gated and shortened. This was observed on an oscilloscope by comparing the injection pulses sent from the OEM ECU, and the injection pulses received by the injectors post-DIM intervention (shown in chapter 6.2). The next step was to set a given injection window, increase the demand, and observe the engine performance. If the injection window is constant, increasing the demand on the engine should have no effect on the engine's output, as only a set amount of fuel is being delivered.

The next feature tested was the timing advance. This was again observed with an oscilloscope by comparing the signals from the timing sensors of the engine to those sent to the OEM ECU from the DIM. When the timing signals are advanced, the phase-shift should be apparent in the waveforms onscreen. After each of the DIM subsystems were tested individually, they were then tested while under control of the DFC. 


\subsubsection{Data Collected}

The raw data collected from LOGINT is saved in a log file, which is simply a list of all monitored variables and their values. A full list of the variables saved can be seen in the LOGINT.SAV file in appendix 5. LOGINT also saves a snapshot of the last pressure trace on cylinder 1 from the PSCOPE program whenever a log is taken. A data point is taken at each speed and load selected once the system has reached an equilibrium (usually approximately 20 seconds after the variable has been changed). After the load/speed of the engine is changed, the system is given 30 seconds for the dynamometer to stabilise before a data point is taken. The tests are performed once the engine has warmed up to keep the tests as controlled as possible.

\subsubsection{Analysis}

To analyse the results, a program written by a previous engineer imports the $\log$ files for a given test (or series of tests) and places them in a spreadsheet which derives values for efficiency, fuel consumption and power output. The spreadsheet can produce charts for graphical analysis of engine performance. For the purposes of this project, the main parameters analysed were power output, fuel substitution and efficiency. The power output of the engine running on dual fuel was compared to the power output when running on diesel, and the differences in total fuel consumption recorded. 


\section{Results}

This chapter details the testing of the main DIM systems under control of the dual fuel controller. These systems include the switching system, timing system, and pilot fuel system.

\subsection{Gating System}

The injection gating system was tested by running the DIM in diesel mode (i.e. no gas injected) and varying the values of the two switching variables: inj.window and inj.dur. The engine was run at a moderate speed of $1600 \mathrm{rpm}$ and the injection duration set to the maximum of $6 \mathrm{~ms}$.

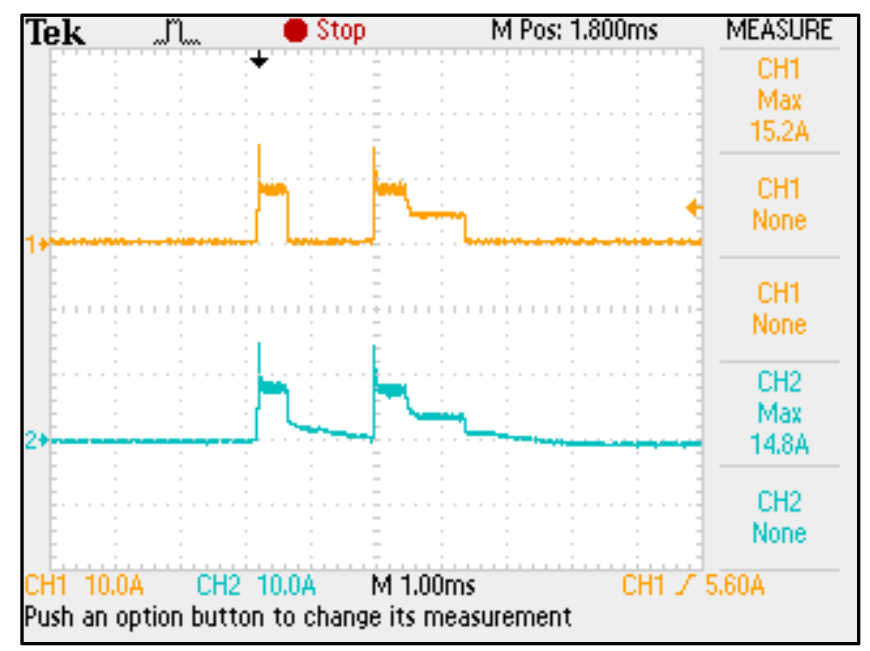

Figure 6.1 Injection window $=20$ degrees

With the injection window set to open at 20 degrees before TDC, both pilot and main injection pulses are allowed to pass through the DIM to the injectors. Figure 6.1 shows that both pulses from the OEM ECU (orange) reach the injectors (blue). PSCOPE records the beginning of injection (BOI) at 18.8 degrees when the pilot pulse appears, as shown in figure 6.2. The peak in cylinder pressure occurs right at (TDC), which is to be expected from the system without intervention.

The multiple peaks in cylinder pressure seen following the main peak are the result of resonant spikes in pressure which occur within the pressure transducer mounting adapter. Because the adapter is a long narrow tube, it is susceptible to resonant fluctuations in pressure during combustion. This issue can be solved by mounting the transducer directly in the cylinder head but this would significantly increase costs. 


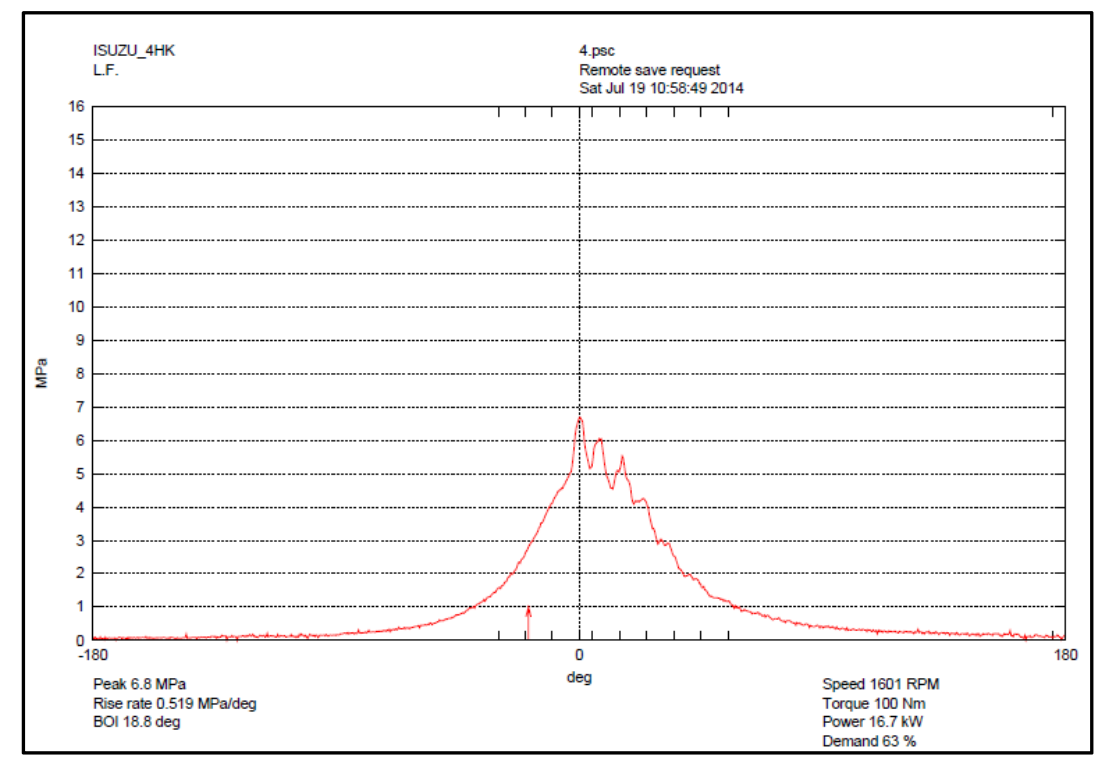

Figure 6.2 PSCOPE trace of engine running on diesel, both injections allowed

With the injection window set to 5 degrees, the window should be closed to the pilot pulse, and open between the pilot and main pulses, letting only the main pulse through to the injector.

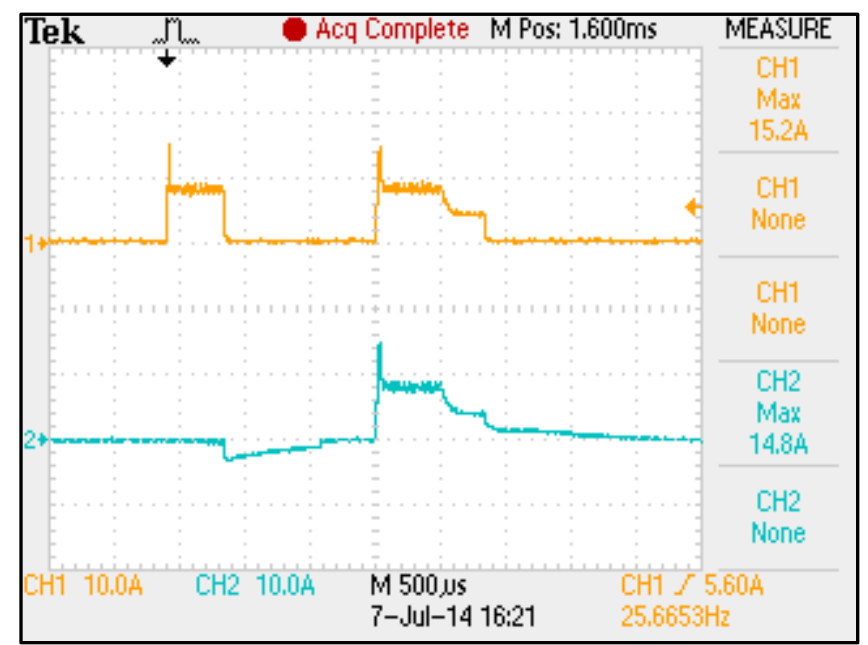

Figure 6.3 Injection window $=5$ degrees

Figure 6.3 shows the removal of the pilot pulse. The orange trace is the current from the OEM ECU, and the blue trace shows the current through the injector. The current briefly goes negative as the OEM injection current through the dummy switches off. It is evident that the current through the injector is only that of the main injection pulse.

PSCOPE records a BOI of 2.2 degrees (figure 6.4), which corresponds to the timing of the main injection pulse. PSCOPE records two peaks, the first at TDC which corresponds to the piston compressing the air and fuel in the cylinder, and the second sharp peak is the actual 
combustion, occurring much later. With the timing so far retarded, the engine runs very inefficiently, and will not be able to ignite gas at the optimum time. To improve this, the timing must be advanced. The effects of the timing system on cylinder pressure is described in section 6.2.

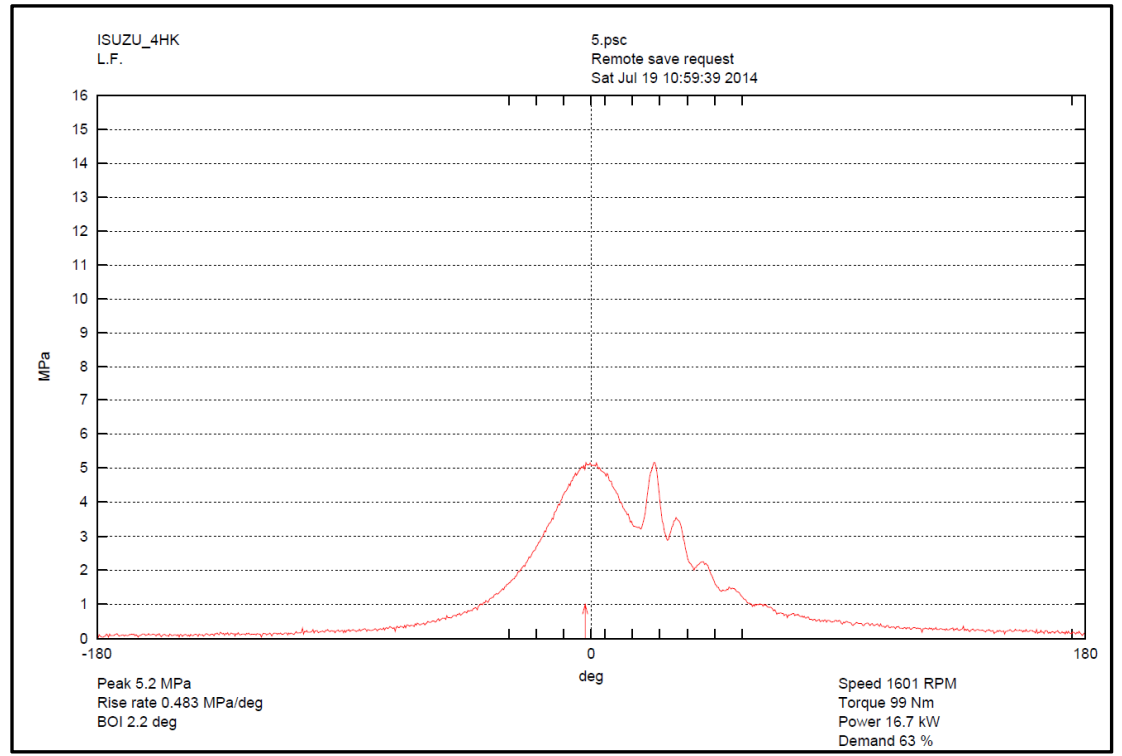

Figure 6.4 PSCOPE trace with first injection removed

If the injection window should open at the same time as the OEM ECU is delivering an injection pulse, the current will begin flowing to the injector as the window is opened. The current from the OEM ECU briefly dips as the DIM switches from the dummy loads to the injectors, producing an inductive kick from the injectors. Figure 6.5 shows what happens when the injection window is set to 10 degrees, approximately 2 degrees after the pilot pulse begins.

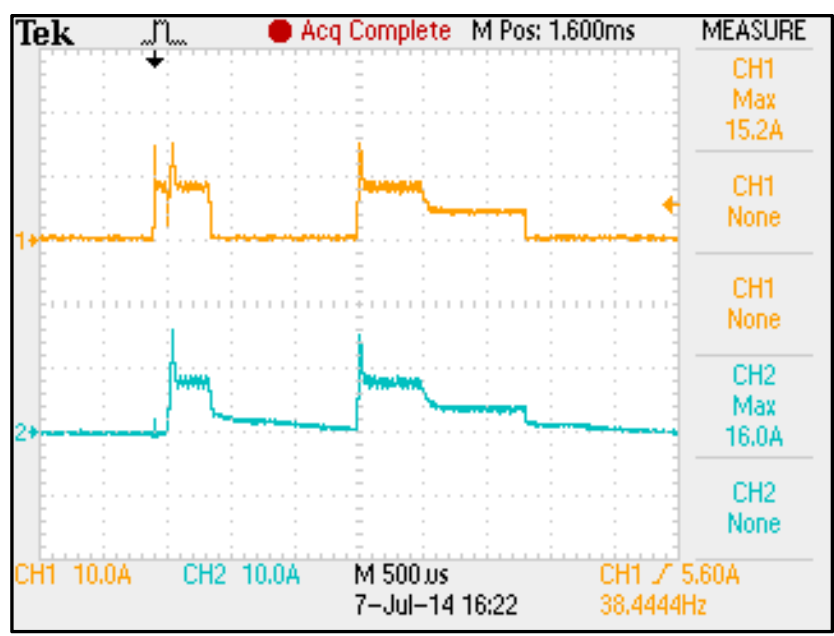

Figure 6.5 Injection window $=10$ degrees 
Figure 6.5 illustrates that the pilot pulse (orange) has been shortened by approximately $100 \mu \mathrm{s}$ (blue). It is important that the injection window only opens between the pilot and main pulses, to reduce the likelihood of knock from having the gas combust before TDC.

\subsection{Timing System}

The timing advance system is an important component of the dual fuel system for controlling the start of ignition of the fuel in the combustion chamber. At higher speeds the timing will need to be advanced in order to ignite the fuel closer to TDC, in order to provide the best power output.

Figure 6.6 shows the input signal from the flywheel sensor (orange) and output to the OEM ECU (blue). One can see that the timing signals are in phase, with the zero crossings at approximately the same point.

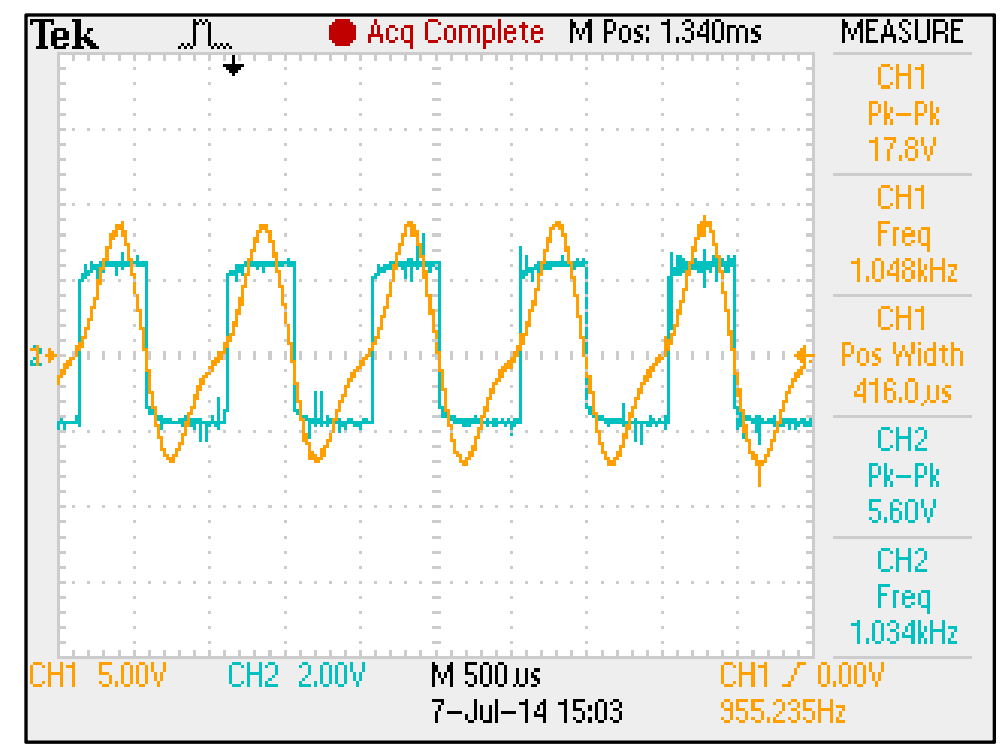

Figure 6.6 Comparison of flywheel sensor input (orange) and DIM output (blue)

Before the timing advance function was tested, the timing of the output signals from the DIM was compared to the input from the sensors to see what delays the DIM intervention has introduced to the system. The engine was run at speeds from 1000 to 2000 RPM and the timing signals into and out of the DIM observed on an oscilloscope. 


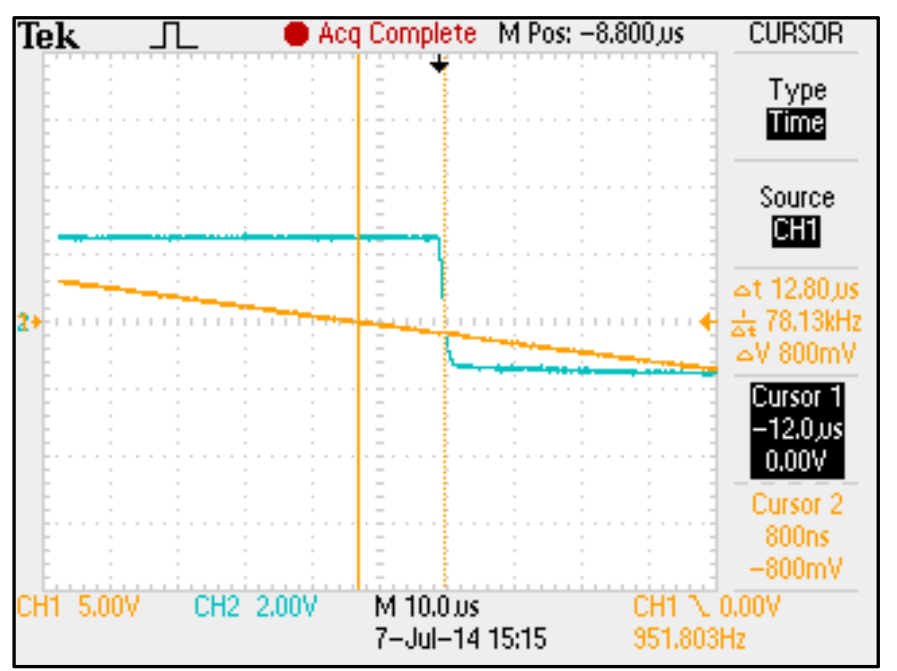

Figure 6.7 Measurement of timing delay on flywheel sensor input/output

Figure 6.7 shows the comparison of the flywheel sensor output (orange) and the DIM output (blue) at $2000 \mathrm{rpm}$. The cursor function on the oscilloscope was used to measure an output delay of $12.8 \mu$ s between zero-crossings. The delay of $12.8 \mu \mathrm{s}$ was observed for all engine speeds, as this is a static delay caused by the microcontroller in the DIM reading in the signal and producing the required output.

As engine speed increases, this delay will become more significant as the timing becomes further retarded. At $1000 \mathrm{rpm}$, a delay of $12.8 \mu$ s corresponds to a lag of only $0.075^{\circ}$, yet at maximum speed $(2000 \mathrm{rpm})$, this becomes $0.15^{\circ}$. A phase shift of $0.15^{\circ}$ is not noticeable in terms of engine operation as engine performance typically only becomes measurably affected at angles greater than half a degree. However, this additional delay factor should not be ignored, and can be compensated for by advancing the timing at higher speeds if required.

The same test was then performed on the cam sensor signals, in order to determine if the delays were the same for both. If the signals are delayed by different amounts the system may not function correctly if the OEM ECU is calculating time-critical information. The input and output signals were measured on an oscilloscope and the rising edge of the cam sensor pulses were compared and measured using the cursor function.

Figure 6.8 shows a delay of $12.2 \mu \mathrm{s}$ between the cam sensor input (orange) and DIM output (blue). This delay is comparable to the delay of the flywheel signal, and is again constant across all engine speeds. A difference of $0.4 \mu$ s between the phase and position sensors corresponds to a difference in phase of only $0.005^{\circ}$, which produces no measurable difference in engine output. 


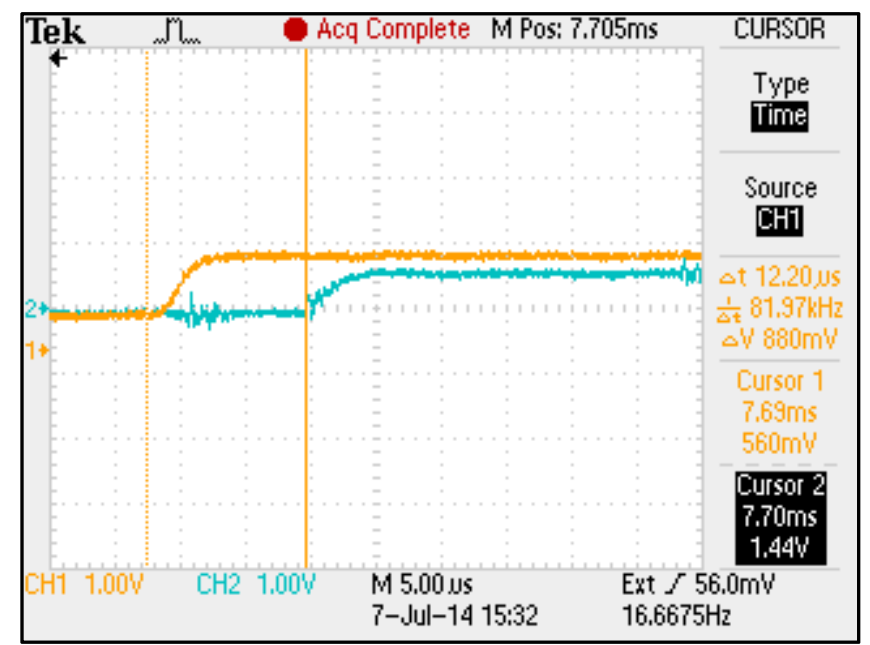

Figure 6.8 Comparison of cam sensor input (orange) and output (blue)

The timing advance system was then tested by increasing the inj.timing variable. In the current software revision, the inj.timing variable only becomes active above 4 degrees, as it was determined in an early specification that smaller advance angles will not be required. In later software revisions, this specification will be amended in order to remove the static delays caused by the timing system.

Testing the timing advance system proved to be challenging, as it was hard to advance the timing signals without the advanced injection causing the engine to surge. With the engine surging, it was impossible to record the speed of the engine and timing delay, both of which need to be kept consistent during the experiment. Therefore during testing the timing was advanced until the engine ran smoothly without surging and the delay in timing signals measured on an oscilloscope. The phase sensor signals were used as the reference for this experiment, because the position waveform will be difficult to measure at timing advances of greater than six degrees.

The timing advance was tested at 5, 9, and 18 degrees, at speeds between 1500 and $1650 \mathrm{RPM}$ as the engine would run smoothly without surging at these points. Table 6.1 on the following page shows the results of this testing.

Table 6.1 shows that the timing system can dynamically adapt to changes in RPM to produce the correct delay for the required advance in timing. All of the measured advance angles were within 0.1 degree of the specified angle, and with such variable measurement methods, this is a promising result. It is assumed that at least some of the error introduced in these results is the product of fluctuations in engine speed between measurements on the oscilloscope. 
Table 6.1 Testing of timing advance system

\begin{tabular}{|l|r|r|r|r|}
\hline RPM & $\begin{array}{l}\text { inj.timing value } \\
\text { (degrees) }\end{array}$ & $\begin{array}{l}\text { Signal delay } \\
(\mu \mathrm{s})\end{array}$ & $\begin{array}{l}\text { Advance angle } \\
\text { (degrees) }\end{array}$ & \multicolumn{2}{|l|}{$\begin{array}{l}\text { Absolute error } \\
\text { (degrees) }\end{array}$} \\
\hline 1656 & 0 & 12 & -0.12 & 0.12 \\
\hline 1506 & 5 & 560 & 5.06 & 0.06 \\
\hline 1644 & 9 & 920 & 9.07 & 0.02 \\
\hline 1652 & 18 & 1800 & 17.8 & 0.07 \\
\hline
\end{tabular}

The timing advance system is used to advance the position of the injection pulses relative to TDC in order to achieve optimal combustion when running on dual fuel. If the timing is too late the combustion will not be efficient (or possibly not even occur at all), and if the timing is too advanced the fuel could detonate before TDC and severely damage the engine.

To test the effectiveness of the timing advance system the pilot pulse was first removed, resulting in late combustion (as seen in figure 6.4, section 6.1). The timing was then advanced and the pressure trace observed on PSCOPE until combustion begins closer to TDC. It can be seen in figure 6.9 that the cylinder pressures increase as the timing is advanced closer to TDC, indicating that the fuel is burning more rapidly and efficiently.

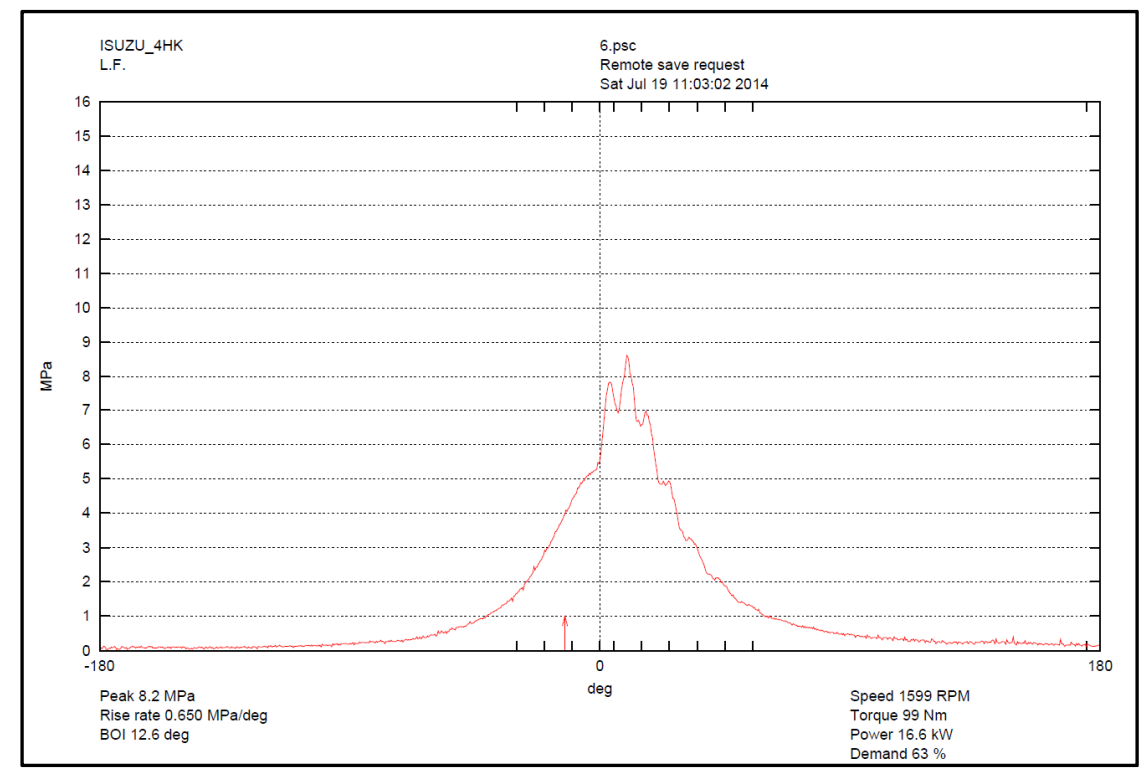

Figure 6.9 First pulse removed, timing advanced 6 degrees 
Figure 6.9 indicates that the timing was advanced 6 degrees, increasing the BOI to 8.2 degrees before TDC (compared to 2.2 degrees as seen in figure 6.4 in the previous section). This shows that the timing can be advanced by the DIM sufficiently to ignite gas introduced by the dual fuel controller.

\subsection{Pilot Fuel System}

With the gating and timing systems tested and working, it is possible to test the diesel reduction system. The pilot fuel system uses both the switching and timing systems to remove the first injection pulse, shorten the main pulse to reduce diesel flow to pilot levels, and advance the timing to ensure the diesel injected will ignite the gas at the correct time.

The pilot system is controlled by the DFC which sends instructions to the DIM, setting the injection window and duration variables as well as the timing advance required. The DFC generates these values from a series of lookup tables which map demand, speed and target diesel flow to injector on-time and timing advance. These fuelling tables (which will be specific to each target engine) are generated by taking thousands of data points across a full load and speed profile of the engine.

For initial testing there is no data available with which to generate a fuel table, so the maximum and minimum injection window values were based on those observed during the engine running on diesel and entered into the table as a starting point. The maximum on-time was set to the maximum of the DIM $(6 \mathrm{~ms})$ and the minimum set to $10 \%$ of this value $(600 \mu \mathrm{s})$. The DGTech program interpolates intermediate values for these variables across varying demand and speed.

To test the pilot fuel system, the engine was set to $900 \mathrm{rpm}$ (20\% demand) with no load. The first injection pulse was removed by setting the injection window to open at $2^{\circ}$, and the second pulse shortened until the engine speed began to decrease. With an injection duration of $1.36 \mathrm{~ms}$, the engine speed was reduced to $860 \mathrm{rpm}$. With the engine running stably at this point, the demand was increased up to $100 \%$, and the engine speed did not increase as the fuel flow was limited by the gating system. Figure 6.10 illustrates how the injector current is limited by the injection window. This demonstrates that a reduction in injector on-time reduces diesel fuel flow. The next step is to measure engine performance on diesel before introducing gas. 


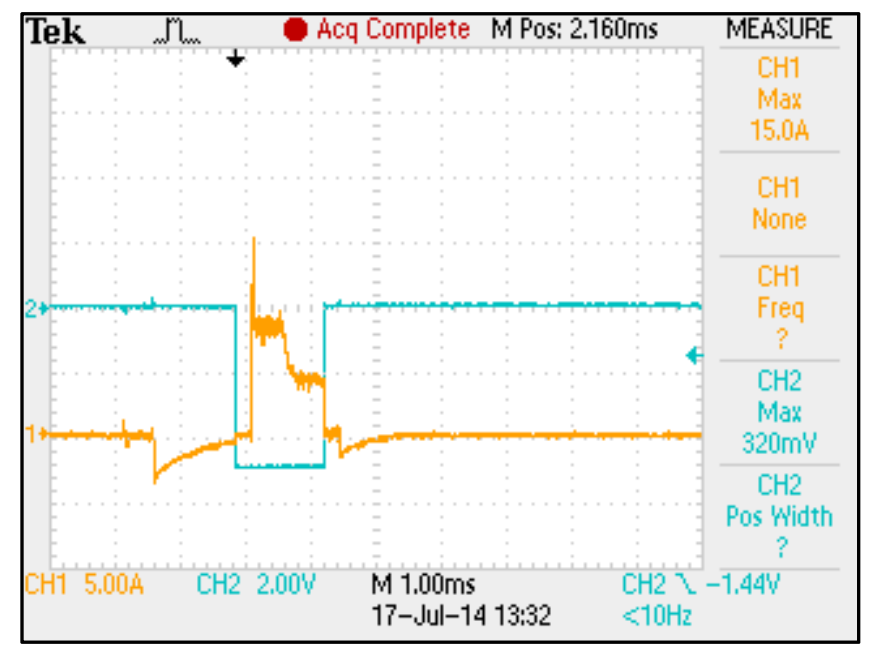

Figure 6.10 Pilot diesel pulse (orange) and injection window (blue)

\subsection{Engine Performance}

\subsubsection{Diesel Operation}

To characterise the engine running on diesel without DIM intervention, the engine was first run at $100 \%$ demand and the applied torque from the dynamometer slowly increased until the speed began to drop. Measurements were taken as torque was further increased until the engine speed was below idle, giving a graph of the maximum torque the engine can produce at each speed (figure 6.11).

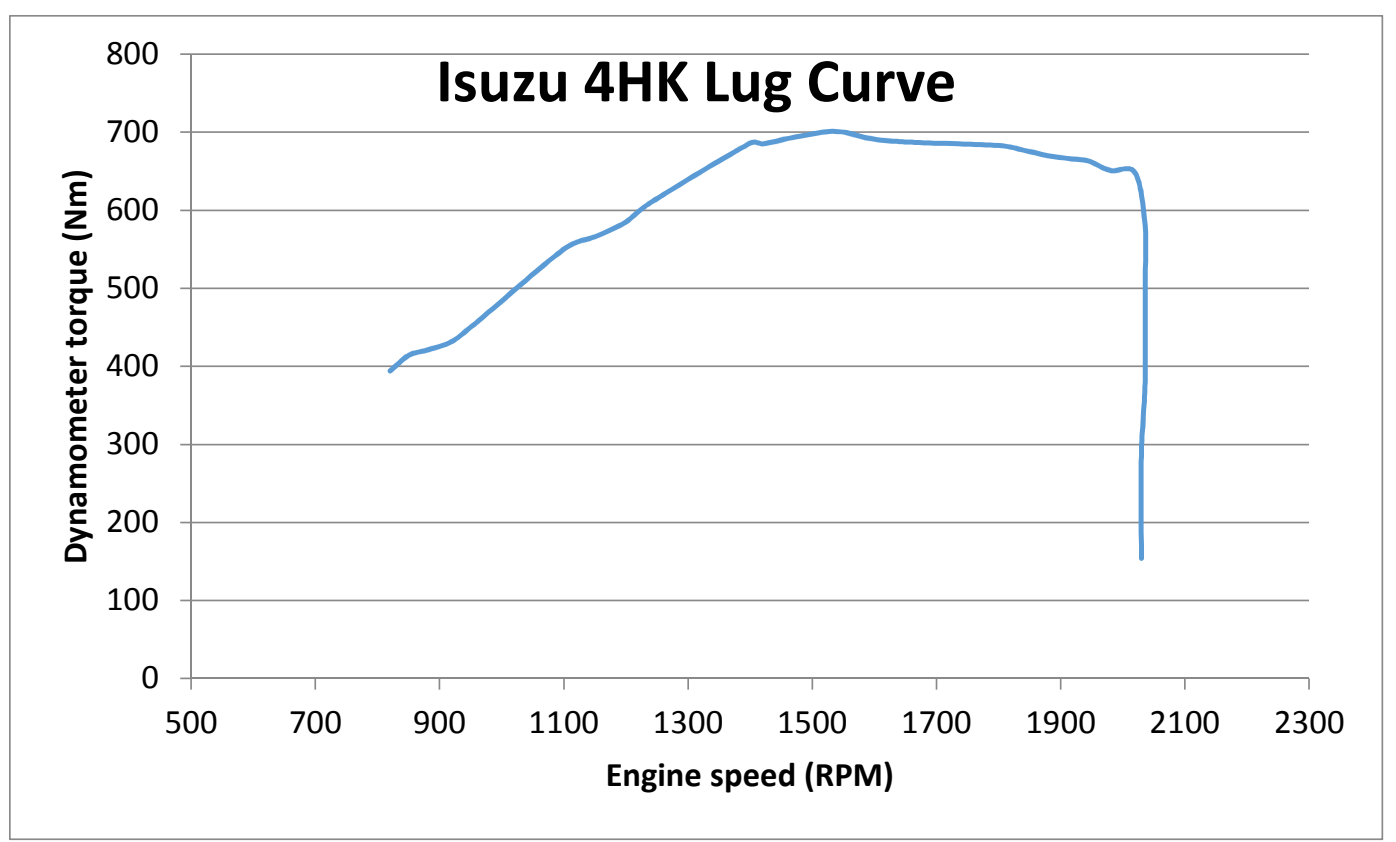

Figure 6.11 Maximum torque curve (lug curve) of Isuzu 4HK running on diesel 
This test was repeated several more times at lower demand settings to produce a series of curves (known as droop curves), showing how the engine output responds to increasing load when the demand is less than $100 \%$, as would be expected under driving conditions. The family of curves produced also provides an indication of how effective the engine governor is at maintaining engine speed for a given demand, as mentioned earlier in section 5.3.3.

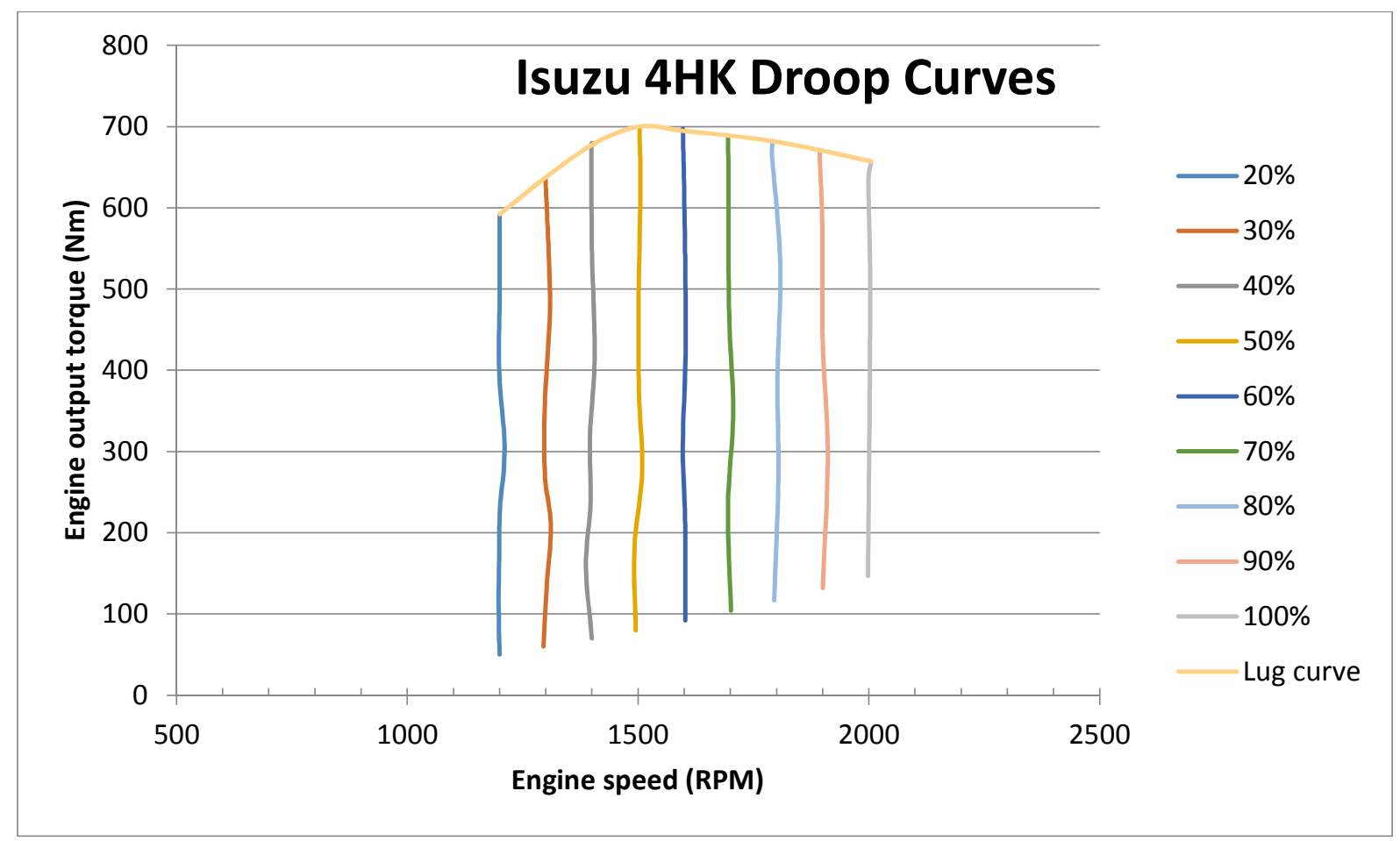

Figure 6.12 Isuzu 4HK droop curves showing governor performance with varied \% load

Figure 6.12 shows the series of droop curves from the $4 \mathrm{HK}$ engine, bounded by the lug curve. The almost vertical droop curves show that the engine governor is very effective at maintaining engine speed right up until the full output torque of $700 \mathrm{Nm}$ is reached.

During the testing of the dual fuel system described in the next chapter, it was found that the engine was producing over $800 \mathrm{Nm}$ of torque when in diesel mode. This result was unexpected, as the engine is not rated for such high power output and should not be able to achieve such a high output torque. This anomalous result turned out to be the result of the switching system increasing the turn-off time of the injectors, (as described in chapter 4.2.3, during the testing of the DIM systems).

By increasing the turn-off time of the injectors, more fuel is being injected into the cylinder than required, and thus the power output of the engine is higher. This is a dangerous result, as it places greater stress on the engine's mechanical systems and could potentially lead to 
engine failure. The switching system in future revisions will use a true bipolar switching system, such as a solid-state relay to eliminate this effect.

The Isuzu 4HK engine was obtained with the knowledge that it was already suffering from wear in the cylinder head, which is a common fault with this engine. To preserve the engine as much as possible, care was taken when running dual fuel tests to not stress the engine too much by keeping cylinder pressures below $14 \mathrm{mPa}$, and to not load the engine past the manufacturer's specifications.

\subsubsection{Dual Fuel Operation}

To measure the power output on dual fuel, the engine was run at $100 \%$ demand and dynamometer load increased until the desired speed was reached. The load on the engine was recorded and fuel consumption measured. The target diesel flow for pilot ignition is then calculated as $10 \%$ of full diesel flow. To achieve pilot diesel flow, the first injection pulse is removed, the timing advanced until combustion occurs near TDC, and the injector on-time slowly reduced until the engine drops in speed. At this point, gas is added to bring the speed of the engine and load back up to the starting point. Diesel is gradually reduced and gas increased until the desired substitution rate is achieved.

Because the Isuzu 4HK engine was already suffering from stress-induced wear, it was decided not to run dual fuel tests at full load. Instead, a load of $400 \mathrm{Nm}$ was chosen as a safe starting point for dual fuel trials. The engine was set to $100 \%$ demand, and the dynamometer set to hold the desired RPM. At this point, the diesel flow was recorded the target pilot flow set to $10 \%$ of this value. Gas was gradually added using the pots box until the torque increased, then the diesel reduced to return to a torque of $400 \mathrm{Nm}$ while ensuring the timing was not too advanced/retarded so as too cause knock or late combustion. Substitution was increased until pilot fuel was achieved or substitution reached $90 \%$.

These tests were performed at 1400, 1600, and $1800 \mathrm{rpm}$ as at these speeds the engine runs most smoothly when on $100 \%$ diesel. Again, these values were chosen to ensure no undue stress was placed on the engine. The first dual fuel tests were conducted at $1600 \mathrm{rpm}$ with the estimated fuel table described in section 5.3.2.

At full load, the engine produces $800 \mathrm{Nm}$ of torque (more than the rated maximum). The first injection pulse was removed, timing adjusted and target diesel flow reduced until the engine 
was producing $400 \mathrm{Nm}$ of torque. At this point, the diesel flow rate was $7.6 \mathrm{gs}^{-1}$, so pilot flow was chosen to be $7.6 \mathrm{gs}^{-1}$.

Table 6.2 shows the gradual substitution of diesel with gas. Gas and diesel flows are measured in $\mathrm{gs}^{-1}$, and also in $\mathrm{kW}$, which is determined by the power density of these fuels at the provided temperatures and pressures respectively. To ensure these values remain consistent, the temperature controller was set to hold both fuel and intake air temperatures to $40^{\circ} \mathrm{C}$.

Table 6.2 Results of diesel substitution

\begin{tabular}{|r|r|r|r|r|r|r|}
\hline $\begin{array}{l}\text { Torque } \\
(\mathrm{Nm})\end{array}$ & \multicolumn{1}{l|}{$\begin{array}{l}\text { inj.dur } \\
(\mathrm{ms})\end{array}$} & $\begin{array}{l}\text { Diesel Flow } \\
\left(\mathrm{gs}^{-1}\right)\end{array}$ & \multicolumn{1}{l|}{$\begin{array}{l}\text { Diesel } \\
(\mathrm{kW})\end{array}$} & $\begin{array}{l}\text { Gas flow } \\
\left(\mathrm{gs}^{-1}\right)\end{array}$ & $\begin{array}{l}\text { Gas } \\
(\mathrm{kW})\end{array}$ & \multicolumn{2}{l|}{$\begin{array}{l}\text { Substitution } \\
\%\end{array}$} \\
\hline 802 & 6.012 & 7.6 & 330 & 0 & 0 & 0 \\
\hline 402 & 1.159 & 3.8 & 163.66 & 0 & 0 & 21.5 \\
\hline 491 & 1.168 & 4.02 & 173.18 & 0.8 & 35.86 & 33.6 \\
\hline 396 & 1.041 & 3.04 & 130.96 & 0.8 & 35.86 & 34.1 \\
\hline 412 & 0.971 & 2.64 & 113.75 & 1.28 & 57.63 & 54.7 \\
\hline 405 & 0.973 & 2.56 & 110.28 & 1.26 & 56.98 & 68.6 \\
\hline 399 & 0.820 & 1.68 & 72.5 & 1.95 & 87.72 & 72.8 \\
\hline 398 & 0.731 & 1.17 & 50.5 & 2.44 & 110.13 & \\
\hline 423 & 0.675 & 1.02 & 43.78 & 2.6 & 117.17 & \\
\hline
\end{tabular}

Figure 6.13 shows how reducing the injector on-time reduces the flow of diesel into the engine to approach pilot levels. It can also be seen how the rate of substitution is inversely proportional to diesel flow. All of the power removed by reducing diesel is now provided by increasing gas input. The general trend of diesel flow as seen in figure 6.13 shows that a pilot fuel level below $10 \%$ will be possible, and substitution greater than $70 \%$ can be achieved.

This test demonstrates that the dual fuel system functions adequately and shows that gas substitution can be performed. However, because the values for injector on-time in the fuel table were too high for the given target diesel flow, the pilot diesel flow target of $0.76 \mathrm{gs}^{-1}$ could not be achieved as the fuel table limited injection on-time to $0.67 \mathrm{~ms}$, giving a 
minimum fuel flow of approximately $1 \mathrm{gs}^{-1}$. The fuel table was updated for the later experiments at 1400 and 1800 by scaling the table by 0.7 to reduce the in $j$. dur values.

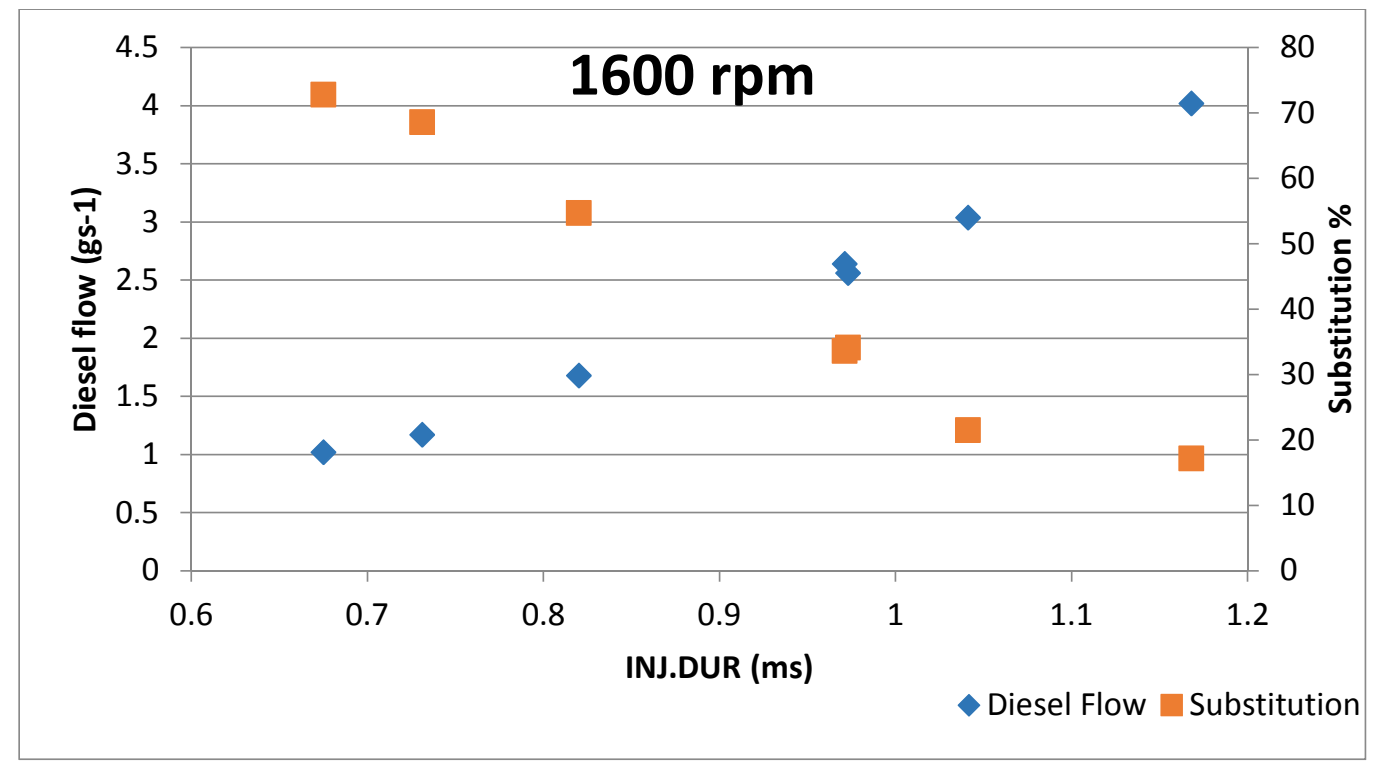

Figure 6.13 Diesel flow and gas substitution vs injection duration at $1600 \mathrm{rpm}$

While the power output of the engine was kept constant throughout the dual fuel tests, it was observed that there was a positive correlation between the substitution percentage and engine efficiency. Figure 6.14 shows the general trend in engine efficiency as substitution increases. This is typical of CNG, as it is a cleaner burning fuel and can be mixed more homogenously with air than diesel.

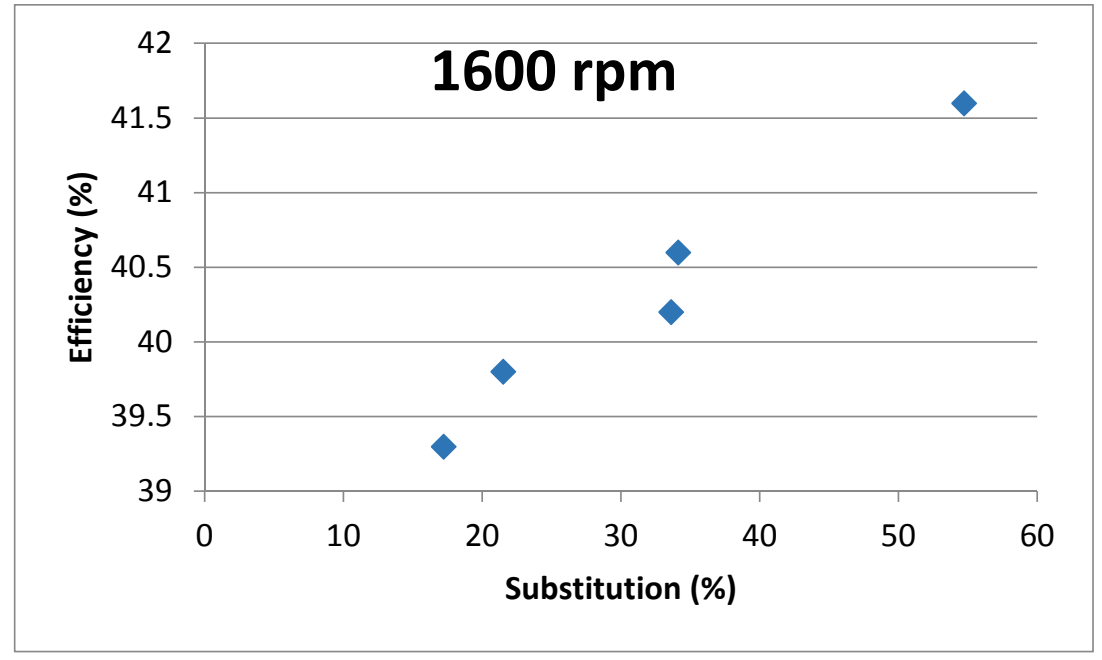

Figure 6.14 Engine efficiency vs substitution at 1600 rpm

The next test was repeated at a speed of $1400 \mathrm{rpm}$, where a substitution of $90 \%$ was achieved with the updated fuel table figures. Figure 6.15 shows how gas and diesel flows change with 
increasing substitution, while torque output remains the same. This shows that the dual fuel system is capable of maintaining performance while replacing up to $90 \%$ of the diesel required.

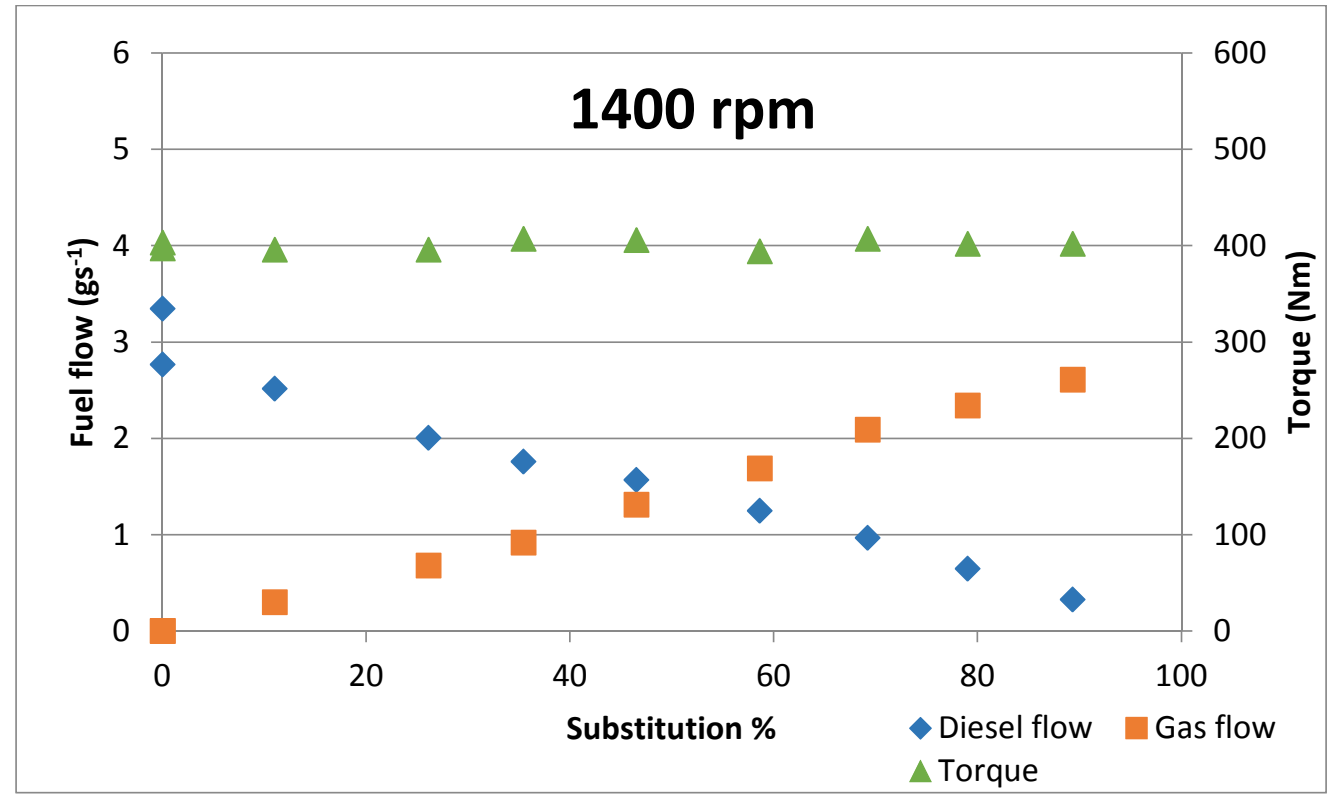

Figure 6.15 Dual fuel flow and torque with increased substitution at 1400rpm

Figure 6.16 shows the cylinder pressure trace at $1400 \mathrm{rpm}$ and $10 \%$ substitution. One can see the start of combustion very clearly, occurring right at TDC. This is an ideal graphical representation of the performance of the dual fuel system, as it shows the operation of the diesel reduction, gas substitution and timing advance functions of the dual fuel system.

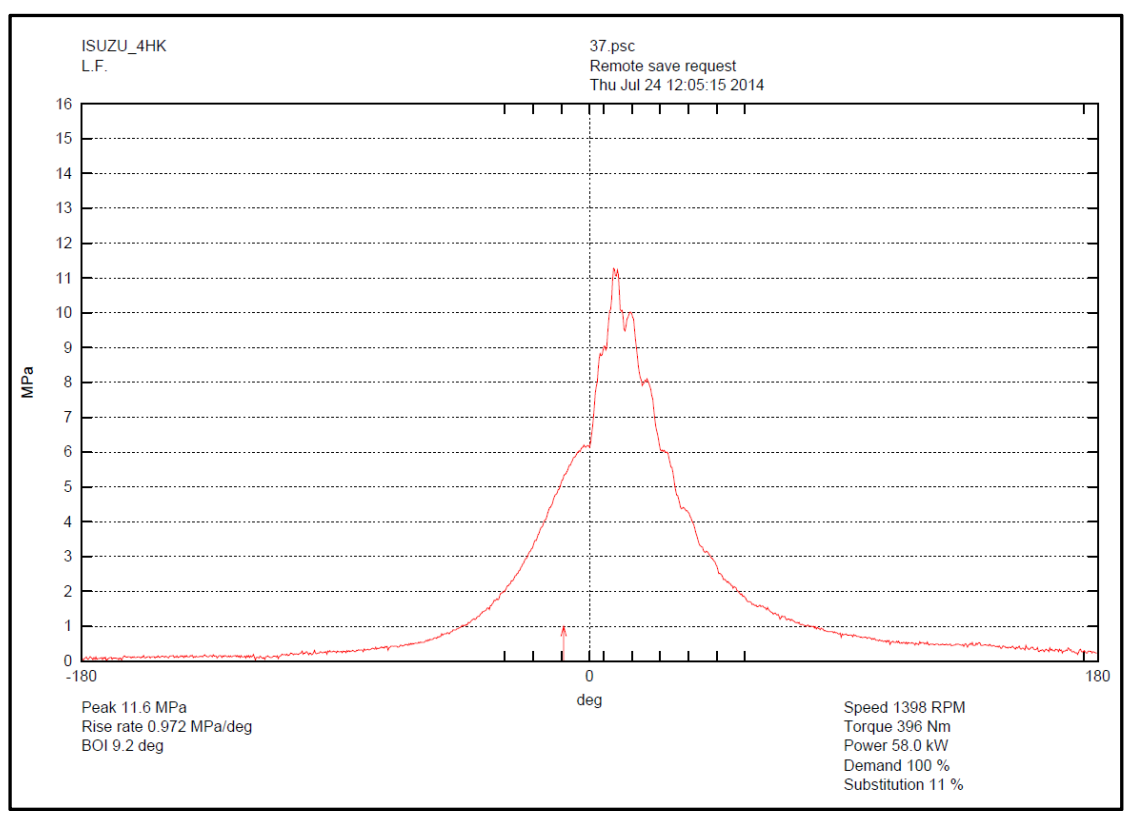

Figure 6.16 Pressure trace during dual fuel operation 
The results of the tests performed at 1600, 1400 and, $1800 \mathrm{rpm}$ were compared, showing the efficiency of the dual fuel system with increased substitution at different speeds. Figure 6.17 illustrates how efficiency on dual fuel almost always exceeds $40 \%$, the baseline for diesel operation. At $1400 \mathrm{rpm}, 50 \%$ efficiency is achieved with $30 \%$ substitution.

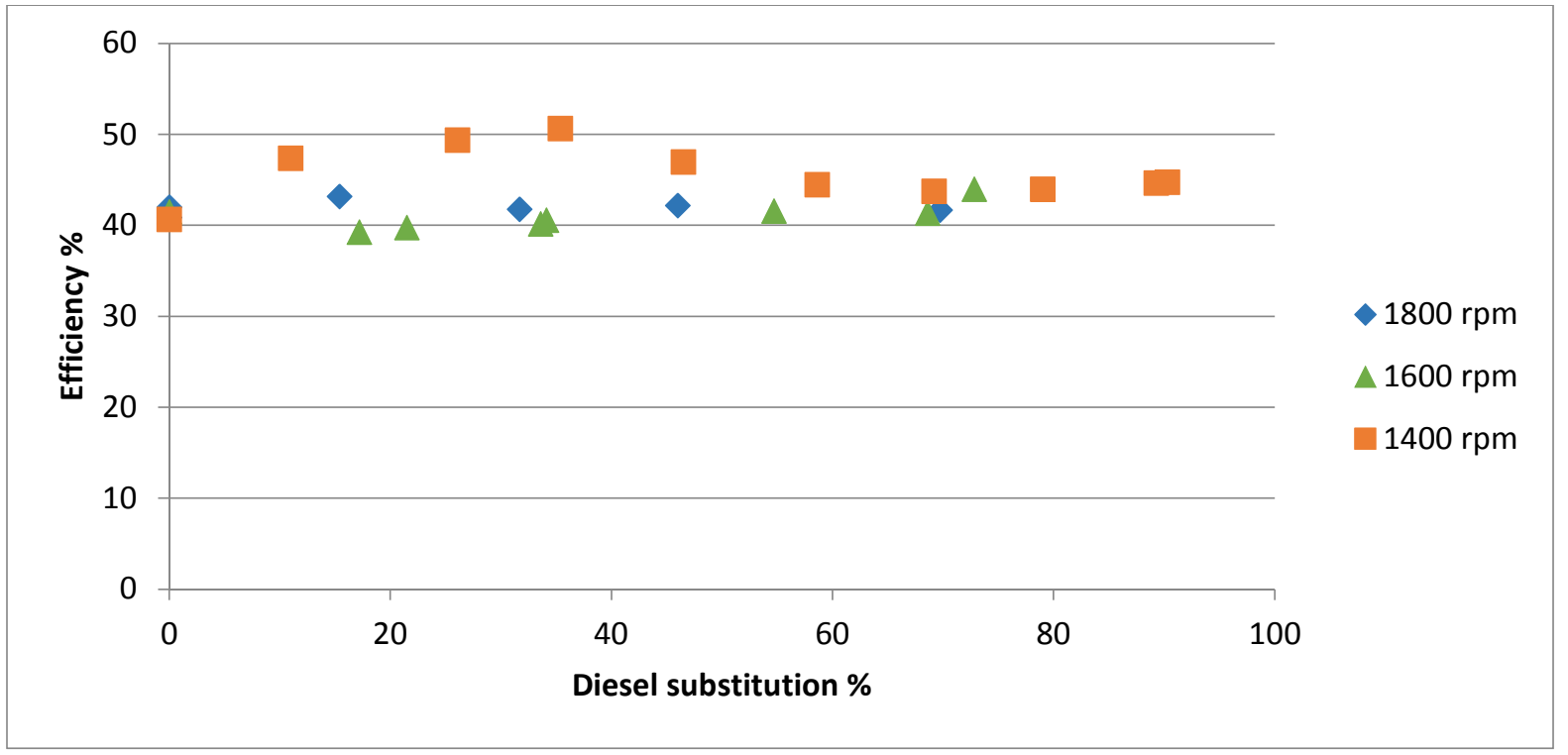

Figure 6.17 Efficiency of dual fuel substitution

The graphs of dual fuel operation at 1600 and $1800 \mathrm{rpm}$ (figures 6.18 and 6.19 respectively) were compared to that of the engine running at $1400 \mathrm{rpm}$ (figure 6.15) to analyse the differences in fuel flow during substitution.

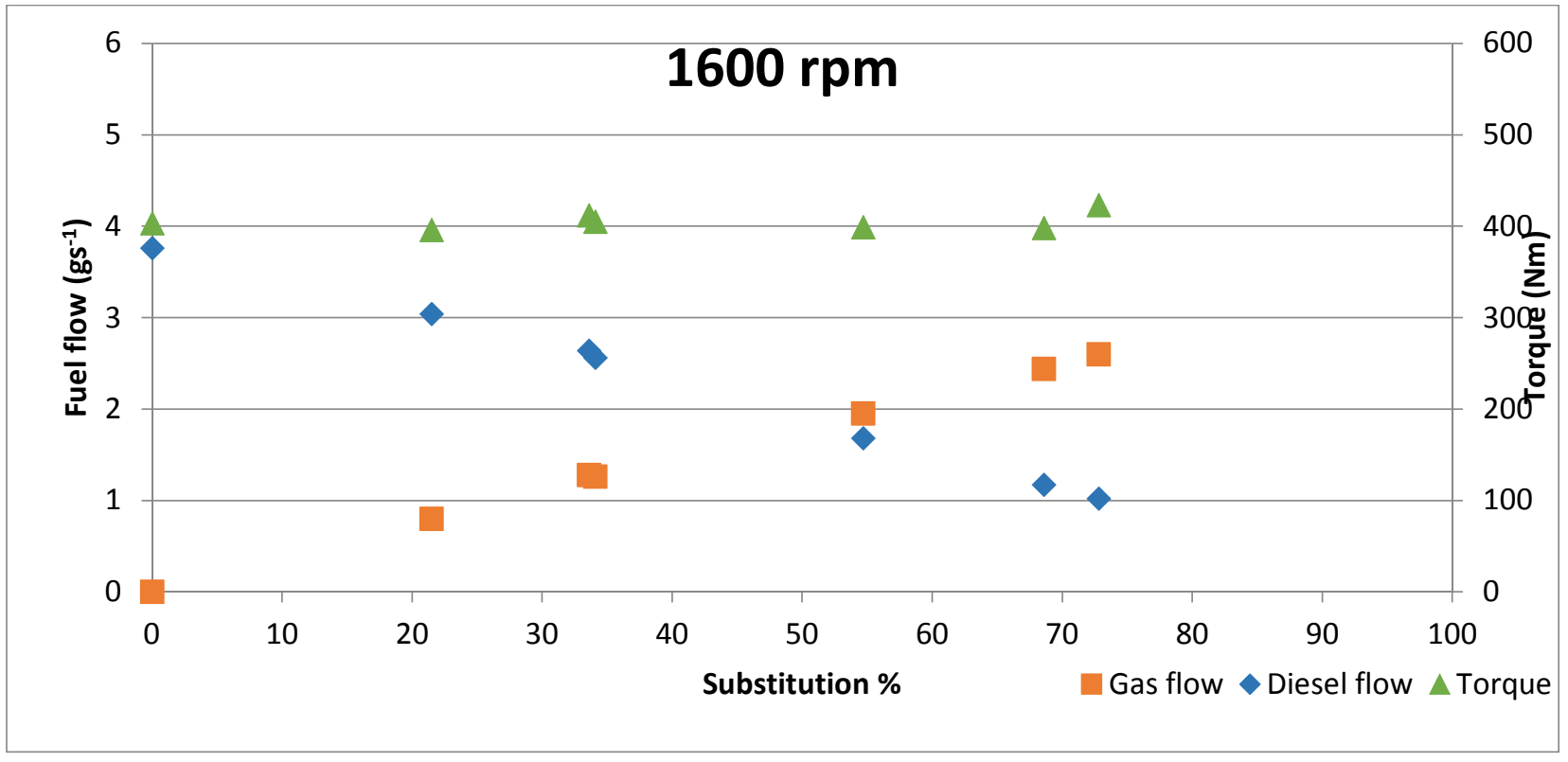

Figure 6.18 Dual fuel flow and torque at $1600 \mathrm{rpm}$ 
The comparison of the three dual fuel tests at 1400, 1600, and $1800 \mathrm{rpm}$ show that for a load of $400 \mathrm{Nm}$, the fuel diesel flow increases approximately $0.5 \mathrm{gs}^{-1}$ for every $200 \mathrm{rpm}$ increase in speed. In each case, $~ 70 \%$ substitution was achieved with a diesel flow of approximately $0.1 \mathrm{gs}^{-1}$. Data points could not be recorded for substitution values of $90 \%$ at 1600 and 1800 rpm, but this was due to fuel supply errors in the test cell. However, it can be seen from figures 6.18 ad 6.19 that the positive trend of the graphs indicate that $90 \%$ substitution is indeed possible at these speeds.

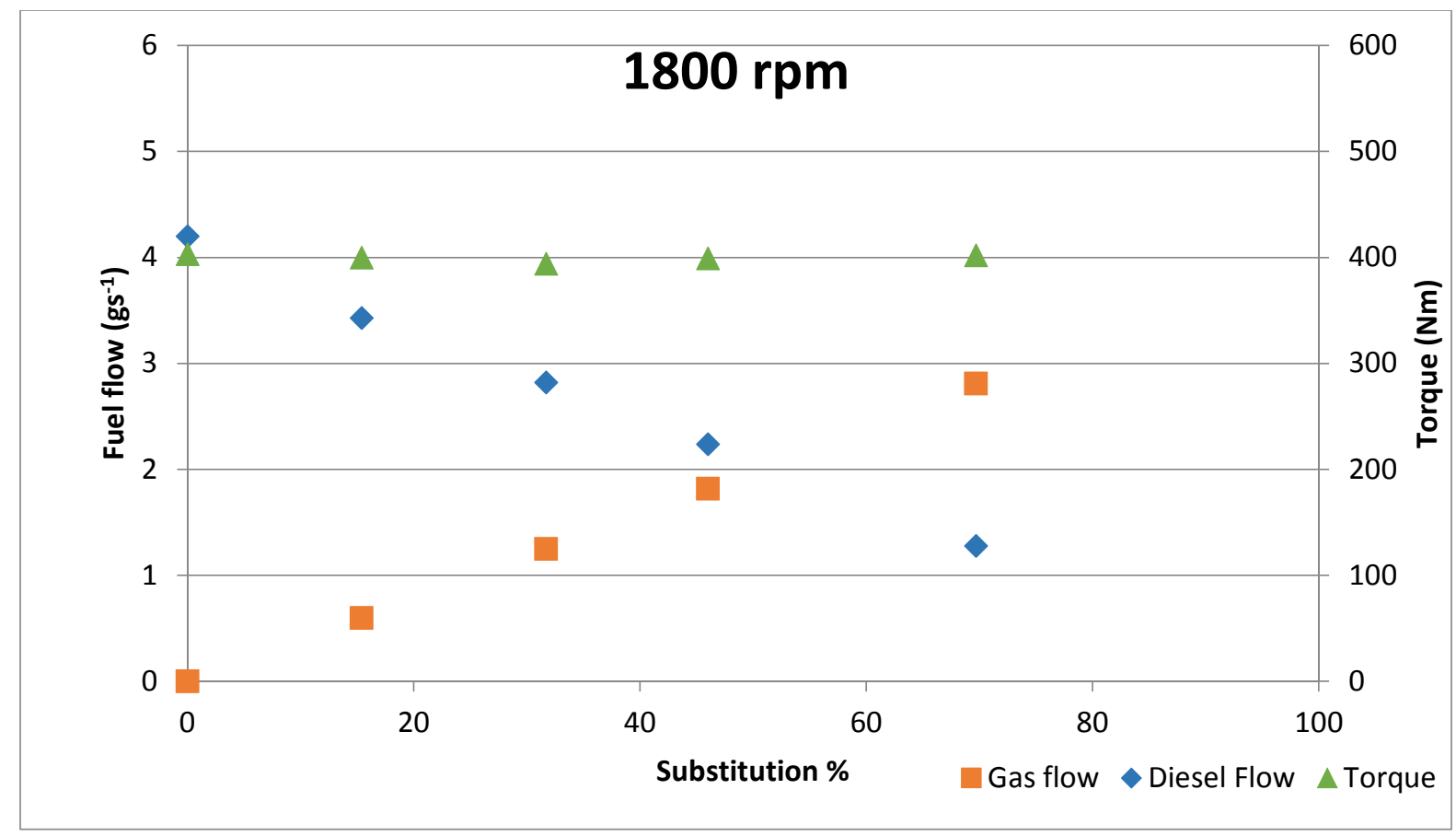

Figure 6.19 Dual fuel flow and torque at $1800 \mathrm{rpm}$

The dual fuel system was tested briefly at full load (800 Nm at $2000 \mathrm{rpm}$ ), and a peak substitution rate of $97 \%$ was achieved. However, at such high substitution rates, the diesel flow through the injector is so low that it can cause the injector nozzles to overheat and deteriorate. The diesel flowing through an injector provides cooling for the injector nozzle and internal components, so a pilot fuel rate of no less than $10 \%$ of normal operation is preferred in order to maintain adequate injector cooling.

At such high substitution rates and advanced timing, the likelihood of knock is also increased and indeed at $97 \%$ substitution the cylinder pressures were so high that they exceeded the range of the PSCOPE program. This can lead to catastrophic engine failure and even melt the top of the pistons. Synchronised control of the injector windowing and advance angle values would have made retarding the timing easier in this situation in order to lower the cylinder 
pressure, as the two are currently controlled independently. However, this test demonstrates that the dual fuel system developed can adequately control the fuelling to well below target pilot levels and maintain engine performance.

After the first initial tests on dual fuel the OEM ECU error logs were read, and no error codes pertaining to the fuel system were found. The only errors recorded were related to missing sensors not required for dual fuel operation. This proves that the injector switching system can mask the intervention of the dual fuel system and meet the OBD requirement set out in section 1.3.

\subsubsection{Summary}

This chapter illustrates that dual fuel operation can be achieved on the Isuzu 4HK1 engine through the use of the prototype dual fuel ECU consisting of a DIM and a DFC. This system was able to substitute up to $97 \%$ of the diesel normally used in standard operation with CNG, maintaining the same power output with increased efficiency, while simultaneously varying the timing of the engine to ensure safe and efficient combustion pressures. 


\section{Conclusion}

\subsection{Evaluation of Results}

The results obtained in chapter 6 show that the prototype Diesel Injection Module can perform the necessary modifications to the required engine signals to allow the engine to be run on dual fuel. Despite several hardware and software issues which still need to be addressed, this prototype does meet the specifications set out in chapter 1 .

The timing system of the engine was designed to advance the timing signals received from the engine with 0.1 degree resolution specified in section 4.2. The dual fuel system exceeds this requirement with recorded timing error of no more than 0.07 degrees, as demonstrated in the results of section 6.2. However, with the timing advance set to 0 degrees, the static delay of $12 \mu \mathrm{s}$ (caused by the timing signal generation) can cause retardation of the timing by up to $0.15^{\circ}$. While this retarding of the timing signals is not noticeable in terms of engine operation, it can compensated for by adding a static timing advance of 0.1 degrees for speeds above $750 \mathrm{rpm}$ which will produce a nominal error of only 0.5 degrees at $1500 \mathrm{rpm}$ (the speed at which the static delay exceeds 0.1 degrees).

The timing system is used to alter the point at which the fuel ignites in the combustion chamber. The timing advance feature of the DIM advances the timing of the main injection pulse sufficiently so that ignition occurs soon after TDC. The effect of the timing system was demonstrated by observing the pressure trace of cylinder 1 while varying the advance angle. The main issue raised by this experiment was the realisation that the injection window and timing advance parameters must be synchronised so an advance in timing also advances the opening of the injection window by the same amount. Without this synchronisation, each variable has to be increased or decreased by small increments while under manual control.

The injector pulses can be accurately masked and shortened by the DIM in order to control fuel flow. Any injection pulses preceding the main injection pulse can be masked by the gating system which opens the injection window at a specified angle before TDC. The length of the main pulse can be reduced with $1 \mu$ s accuracy to decrease the delivery of diesel to pilot levels, thus meeting the specifications set out in section 1.3. The dummy loads mask the presence of the DIM, and thus do not cause the OEM ECU to generate any error codes which may affect its output characteristics. When the diesel is reduced without any gas being 
introduced, the engine will slow down as the fuel is no longer sufficient to generate the power required. Increasing the demand to the OEM ECU at this point will not increase the speed of the engine as it is being limited by the injection gating system.

The engine was initially tested on $100 \%$ diesel fuelling to establish the overall power output of the engine under normal operation. The engine was run at full demand and the load increased to produce a graph of the maximum power the engine can produce at all speeds. The demand was then decreased incrementally and the same test performed to produce a family of droop curves showing how the engine governor responds to an increase in load. These tests established the upper limits for the engine output when running on dual fuel, and described how the engine should operate under different load conditions. The results of section 6.4 .1 show the tests performed at $10 \%$ increments in demand, demonstrating that the engine has a very effective governor.

The injector current pulses can also be modified by the DIM to reduce the amount of diesel injected into the cylinder. This is achieved with an accuracy of $1 \mu$ s allowing the DIM to reduce the normal amount of diesel injected by up to $97 \%$. This is a higher reduction than is necessary for extended running on dual fuel, as a $90 \%$ reduction is the target maximum.

The dual fuel system was tested at various loads and speeds, with the main substitution tests performed at 3 different speeds with moderate load to show the trend of diesel flow and substitution with varying injector on-time of the DIM. The three main test points were chosen as examples of the several hundred data points that would required to generate a complete fuel table for this engine. Time restraints meant that a full fuel table could not be generated, but the tests described in section 6.4.2 have shown that dual fuel operation can be performed by the DIM and DFC. The maximum fuel substitution achieved was $97 \%$, which is an encouraging result given that the target substitution is $90 \%$. Substitution will be limited to $90 \%$ however, in order to preserve the integrity of the injectors which rely on diesel flow for internal cooling.

Overall, the results detailed in chapter 6 show that the dual fuel system developed is capable of controlling the timing and fuelling of an Isuzu $4 \mathrm{HK}$ common rail engine, and can successfully run under load on dual fuel with no loss in output power. 


\subsection{Future Work}

There are several modifications required to improve the performance of the current dual fuel system prototype. First is a comprehensive fuel table with injection duration times across all speed and demand values. Updating the fuel table will allow the target pilot fuel flow to be reached with greater ease. These values can be obtained experimentally and are not difficult to acquire, but will require several weeks of intensive testing.

The next minor change required for the DIM is to synchronise the injection window angle with the timing advance angle. Currently, when the injection window is set (for example to open at 5 degrees before TDC), and the timing is advanced 10 degrees, the injection window will still open at 5 degrees, meaning the injection pulses from the OEM ECU will have occurred before the injection window even opens. The injection window needs to be locked in with the timing advance so they move together with the generated engine signals, not the engine itself.

Another software modification to be investigated in future is the implementation of a timing retard function. Currently, the first injection pulse is rejected and the timing advanced to bring the injection pulse to before TDC. However, during testing it was theorised that the second pulse could be gated and the timing retarded to ignite the fuel closer to TDC. This modification may make dual fuel running easier as the first injection pulse does not increase in size throughout engine operation so this may be an easier way to obtain pilot diesel flows. This system will not work however, if the first injection pulse is too short to provide pilot fuel, which will be tested during implementation.

Currently, the switching hardware of the DIM increases the turn-off time of the injector and thus the overall fuel flow. To rectify this, the next prototype will have a true bipolar switching element such as a solid state relay to prevent this from occurring. This will allow more precise control of the fuel flow, prevent over fuelling and ensure the dual fuel system does not exceed manufacturer's specifications when in diesel mode.

To obtain even more precise control over fuel delivery, it may be necessary to control the common rail pressure. Since the fuel delivery is both a function of injector on-time and rail pressure, keeping the rail pressure constant while in dual fuel mode may allow the DFC to obtain pilot fuel flow more easily. To test this, a PID controller is currently being implemented on the DIM to read the current rail pressure, and amplify this signal before 
sending it to the OEM ECU. The ECU will then respond by lowering the rail pressure until the target set by the DFC is obtained. This addition is not complete at the time of writing, and is currently being implemented by an engineer at DieselGas International.

To improve efficiency and potentially reduce peak cylinder pressures and emissions, the auxiliary functions of the engine such as EGR, VGT, and swirl could be controlled to achieve better combustion performance. These additions will not be implemented in the next prototype, but is a potential optimisation to be added in future iterations.

The next prototype will merge the DFC and DIM onto a single circuit board. This will make the dual fuel system one single unit and eliminate the requirement for a wiring loom between the two. The first revision of this prototype will likely still use ST10 microcontrollers, but future revisions are likely to utilise a new microcontroller which can control both functions. Another proposed addition to the new prototypes is a modular output stage for the various engine signals (i.e. phase, position, rail pressure) with different modules for different brands of sensor. This requirement was suggested after noticing the difference in cam sensor outputs between the WL-C engine of the BT-50 which uses a Bosch fuel system and the Isuzu 4HK1 which uses a Denso system. By having different modular output stages for different fuel system manufacturers, the entire ECU will not have to be redesigned to suit a new target engine, making the dual fuel ECU truly generic.

\subsection{Summary}

The dual fuel ECU developed in this project is able to achieve diesel substitution of up to 97\%. This exceeds the target substitution rate of $90 \%$, and shows that the dual fuel ECU created can be applied to common rail diesel engines. The ECU developed is capable of manipulating engine timing, diesel flow and gas flow dynamically under load.

The system developed is based off an existing DieselGas system and is capable of performing as well as the DieselGas product. By requiring no modification to the original engine at all, this system is easier to implement than the previous DieselGas dual fuel systems which require physical modification to the injector pump. Any common rail diesel engine with solenoid injector valves can be modified to accept this dual fuel system, with the only modification required being the connectors placed in the wiring loom for the timing signals. 
This prototype dual fuel ECU has demonstrated that dual fuel conversion is possible for electronically controlled common rail diesel engines. DieselGas International are now using the technology developed in this project in their first commercial prototype. This prototype is scheduled to be in commercial development by DieselGas in the next 12 months, operating in the US, India and Thailand. 


\section{Bibliography}

[1] Clean Air Power, "How it Works," CLean Air Power, 2013. [Online]. Available: http://www.cleanairpower.com/howitworks.html. [Accessed November 2013].

[2] NGV Global, "New Zealand Harnesses Biomethane for Waste Collection Vehicle Demonstration," $\quad$ November $2010 . \quad$ [Online]. Available: http://www.ngvglobal.com/new-zealand-harnesses-biomethane-for-waste-collectionvehicle-demonstration-1130. [Accessed June 2014].

[3] Delphi, "Worldwide Emissions Standards, Passenger Cars and Light Duty Vehicles," 2013. [Online]. Available: https://delphi.com/pdf/emissions/Delphi-Passenger-CarLight-Duty-Truck-Emissions-Brochure-2012-2013.pdf. [Accessed July 2014].

[4] S. Bennett, Medium/Heavy Duty Truck Engines, Fuel \& Computerised Management Systems, Delmar Learning, 2004.

[5] T. Takaishi, A. Numata, R. Nakano and K. Sakaguchi, "Approach to High Efficiency Diesel and Gas Engines," Mitsubishi Heavy Industries, Ltd. Technical review, 2008.

[6] H. Reid, "Improvements in and Relating to Internal Combustion Engines". New Zealand Patent 204,357, 25 May 1983.

[7] F. DeLuca, "History of Fuel Injection," in Fuel Injection and Controls for Internal Combustion Engines, New York, Simmons-Boardman, 1962, pp. 1-9.

[8] Encyclopaedia Britannica Inc., "Four Stroke Cycle," 2014. [Online]. Available: http://kids.britannica.com/comptons/art-89315/An-internal-combustion-engine-goesthrough-four-strokes-intake-compression. [Accessed June 2014].

[9] B. Sendyka and M. Cygnar, "Stratified Charge Combustion in a Spark-Ignition Engine With Direct Injection System," in Advances in Internal Combustion Engines and Fuel Technologies, InTech, 2013, pp. 85-115. 
[10] T. J. Weathers and C. Hunter, Diesel Engines for Automobiles, Small Trucks and Small Tractors, Prentice-Hall Inc., 1981.

[11] Bosch GmbH, "Mechanical Inline Jerk Pump Cutaway," 2014. [Online]. Available: http://www.bosch-automotivetechnology.us/media/db_application/stage_components/ powertrain/reihenspritzpumpe 1.jpg. [Accessed June 2014].

[12] S. Clever, "Common Rail System Schematic," 2014. [Online]. Available: http://www.sebastian-clever.de/Alt/images/CommonRailSystem_Web.jpg. [Accessed June 2014].

[13] G. Stumpp and M. Ricco, "Common Rail - An Attractive Fuel Injection System for Passenger Car Diesel Engines," in Fuel Spray Technology and Applications: 1996 International Congress and Exposition, Detroit, Society of Automotive Engineers, Inc., 1996, pp. 183-191.

[14] M. F. Russell, G. Greeves and N. Guerrassi, "More Torque, Less Emissions and Less Noise," in Advances in Diesel Fuel Injection and Sprays, Detroit, Michigan, Society of Automotive Engineers, 2000.

[15] W. O. Weernink, "Injector wars: piezo vs. solenoid," Automotive News Europe, 13 November 2006. [Online]. Available: http://europe.autonews.com/article/20061113/ ANE/61109031/injector-wars:-piezo-vs.-solenoid. [Accessed July 2014].

[16] H. K. Suh, S. W. Park and C. S. Lee, "Effect of piezo-driven injection system on the macroscopic and microscopic atomization characteristics of diesel fuel spray," Fuel, vol. 86, pp. 2833-2845, 2007.

[17] Oak Ridge National Laboratory, "Microstructural Evolution of EGR Cooler Deposits," Oak Ridge, 2009.

[18] M. Zheng, G. T. Reader and J. G. Hawley, "Diesel Engine Exhaust Gas Recirculation A Review on Advanced and Novel Concepts," Energy Conversion and Management, no. 45, pp. 883-900, 2004. 
[19] H. A. Soliman, G. H. Abd Alla, O. A. Badr and M. F. Abd Rabbo, "Effect of pilot fuel quantity on the performance of a dual fuel engine," Energy Conversion \& Management, no. 41, pp. 559-572, 2000.

[20] R. G. Papagiannakis and D. T. Hountalas, "Combustion and exhaust emission characteristics of a dual fuel compression ignition engine operated with pilot diesel fuel and natural gas," Energy Conversion and Management, no. 45, pp. 2971-2987, 2004.

[21] A. Paykani, R. Khoshbakhti Saray, A. M. Kousha and M. T. Shervani Tabar, "Performance and Emission Characteristics of Dual Fuel Engines at Part Loads Using Simultaneous Effect of Exhaust Gas Recirculation and Pre-Heating of In-let Air," International Journal of Automotive Engineering, vol. 1, no. 2, pp. 53-67, 2011.

[22] F. Königsson, “Advanging the Limits of Dual Fuel Combustion,” KTH Royal Institute of Technology, 2012.

[23] H. Gurgenci and S. M. Aminossadati, "Investigating the use of Methane as Diesel Fuel in Off-Road Haul Road Truck Operations," Journal of Energy Resources Technology, vol. 131, no. 3, pp. 032202.1-032202.9, 2009.

[24] “Convert a Diesel Generator to Run Dual Fuel with Gas," Dual Fuel Organization, 2012. [Online]. Available: http://www.dualfuel.org/technology/dual-fuel-faq/. [Accessed 2013].

[25] Fairchild Semiconductor, "3N10L Mosfet datasheet," September 2000. [Online]. Available: http://www.sm0vpo.com/_pdf/FQ/FQP33N10L.pdf. [Accessed August 2013].

[26] CUI Inc., "CUI VBT1 Datasheet," [Online]. Available: http://www.cui.com/ product/resource/vbt1-smt.pdf.

[27] Vishay Intertechnology, "Vishay Intertechnology VO3120 gate driver datasheet," October 2012. [Online]. Available: http://www.vishay.com/docs/81314/vo3120.pdf. [Accessed August 2013]. 
[28] International Rectifier, "IRS2117 gate driver datasheet," February 2009. [Online]. Available: http://www.irf.com/product-info/datasheets/data/irs2117pbf.pdf. [Accessed August 2013].

[29] Analog Devices Inc., "ADuM7234 isolated gate driver datasheet," February 2013. [Online]. Available: http://www.analog.com/static/imported-files/data_sheets/ ADuM7234.pdf. [Accessed August 2014].

[30] Allegro Micro Systems L.L.C., “Allegro ACS711 current sensor datasheet,” July 2013. [Online]. Available: http://www.allegromicro.com/ /Media/Files/Datasheets/ACS711Datasheet.ashx. [Accessed August 2013].

[31] ST Microelectronics, "ST10 Microcontroller Datasheet," 2013. [Online]. Available: http://www.st.com/web/en/resource/technical/document/datasheet/CD00157816.pdf. [Accessed August 2013].

[32] Maxim Integrated Products Inc., "MAX 941-944 High Speed Comparator Datasheet," March 2009. [Online]. Available: http://datasheets.maximintegrated.com/en/ds/ MAX941-MAX944.pdf. [Accessed August 2013].

[33] CUI Inc., "DC-DC Converter Datasheet," 9 September 2013. [Online]. Available: http://www.cui.com/product/resource/vbt1-smt.pdf. [Accessed October 2013].

[34] Kistler Instrumente, Kistler 6123 Datasheet.

[35] Pbroks13, "Dynamometer," 2008. [Online]. Available: http://upload.wikimedia.org/ wikipedia/en/2/29/Dyno_schematic.GIF. [Accessed June 2014].

[36] Denso, "Service Manual, Common Rail System for ISUZU 4HK1/6HK1 Type Engine,” February 2004. [Online]. Available: http://www.diesel-art.com/manuales/ izuzu_4hk1.PDF. [Accessed July 2014]. 


\section{Appendix}

\section{Appendix 1 Injector Driver Code}

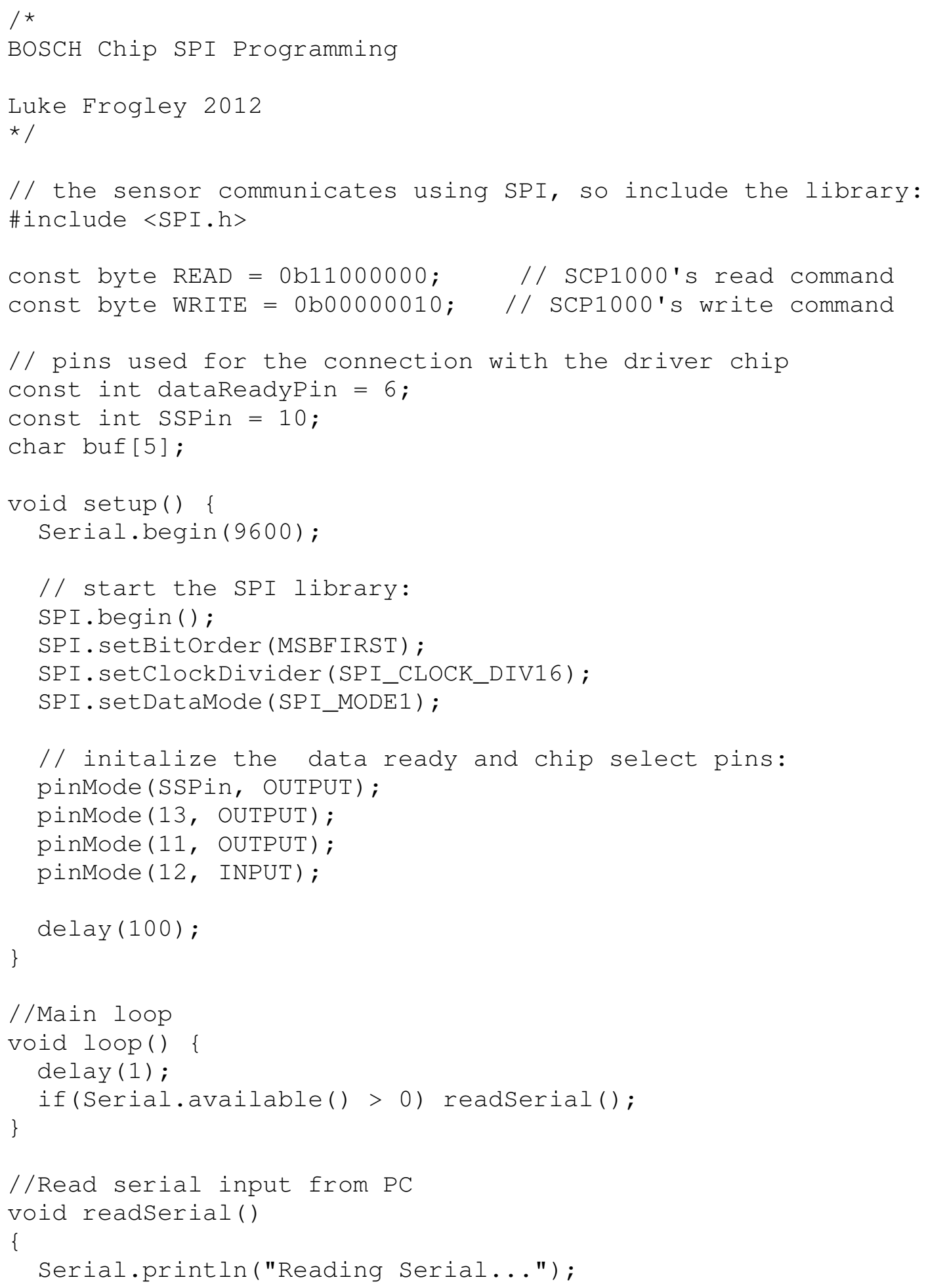




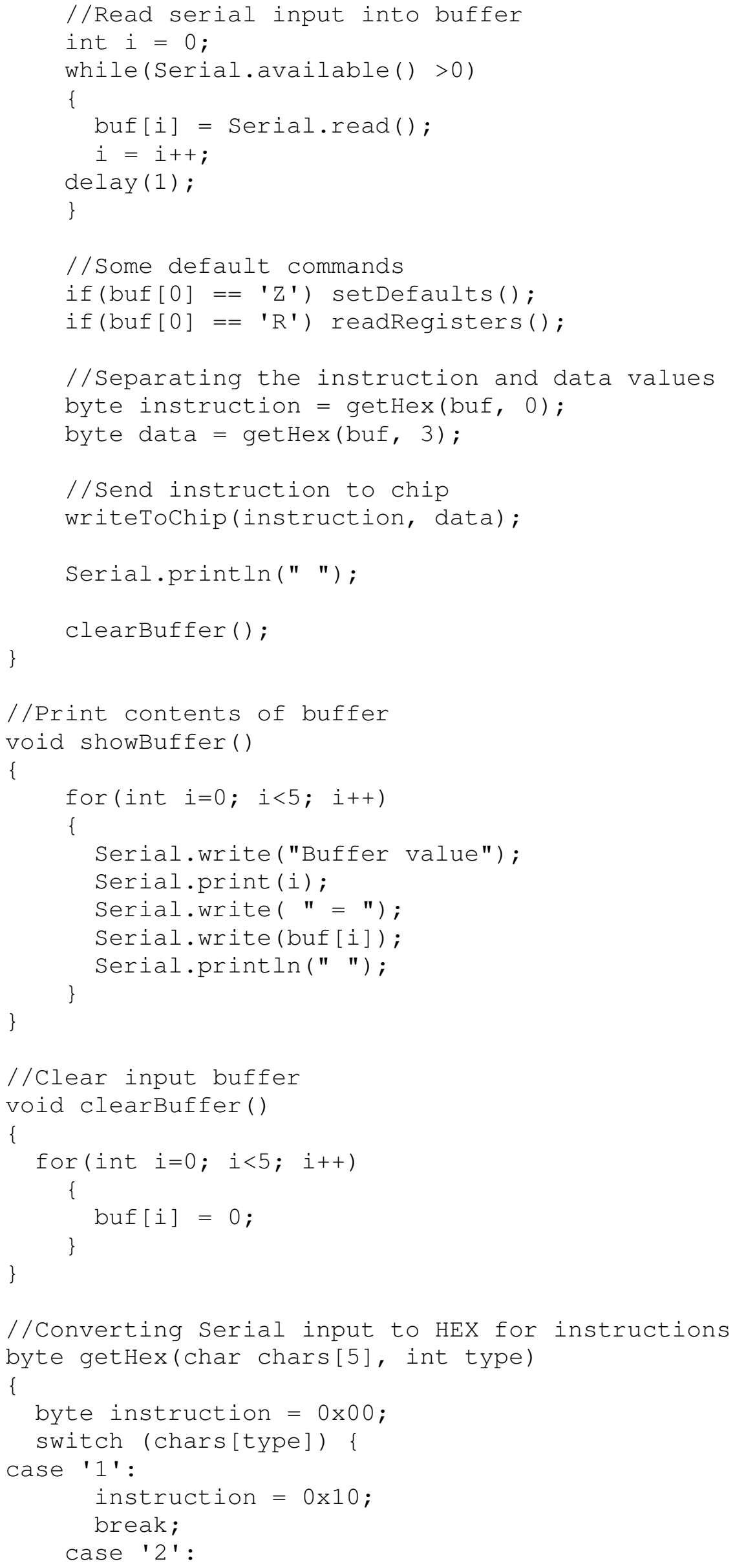




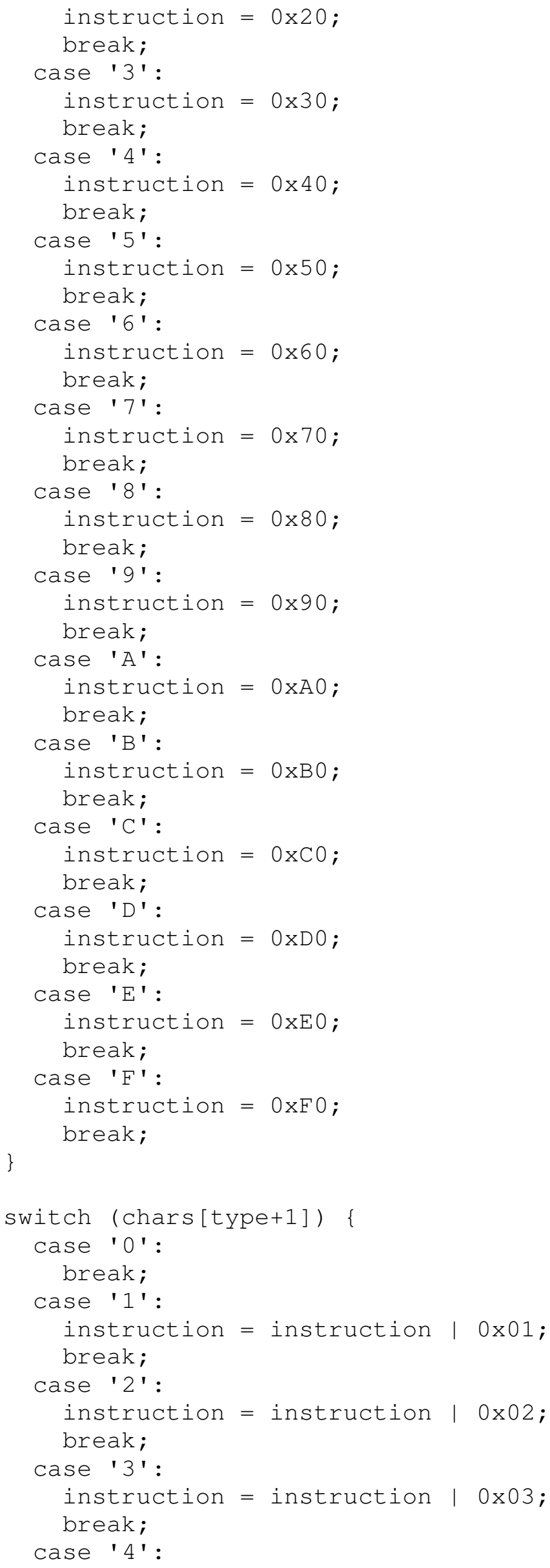




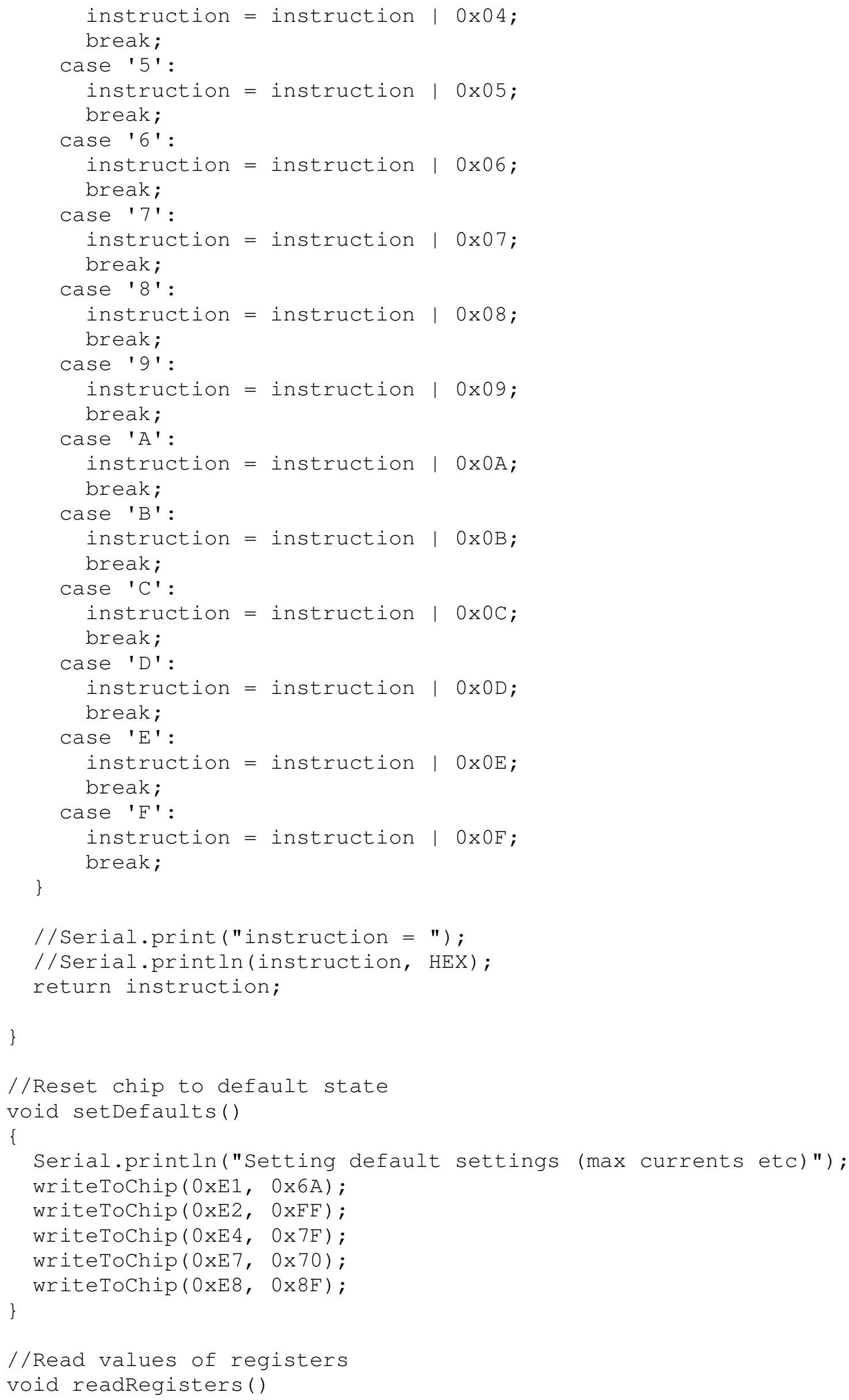




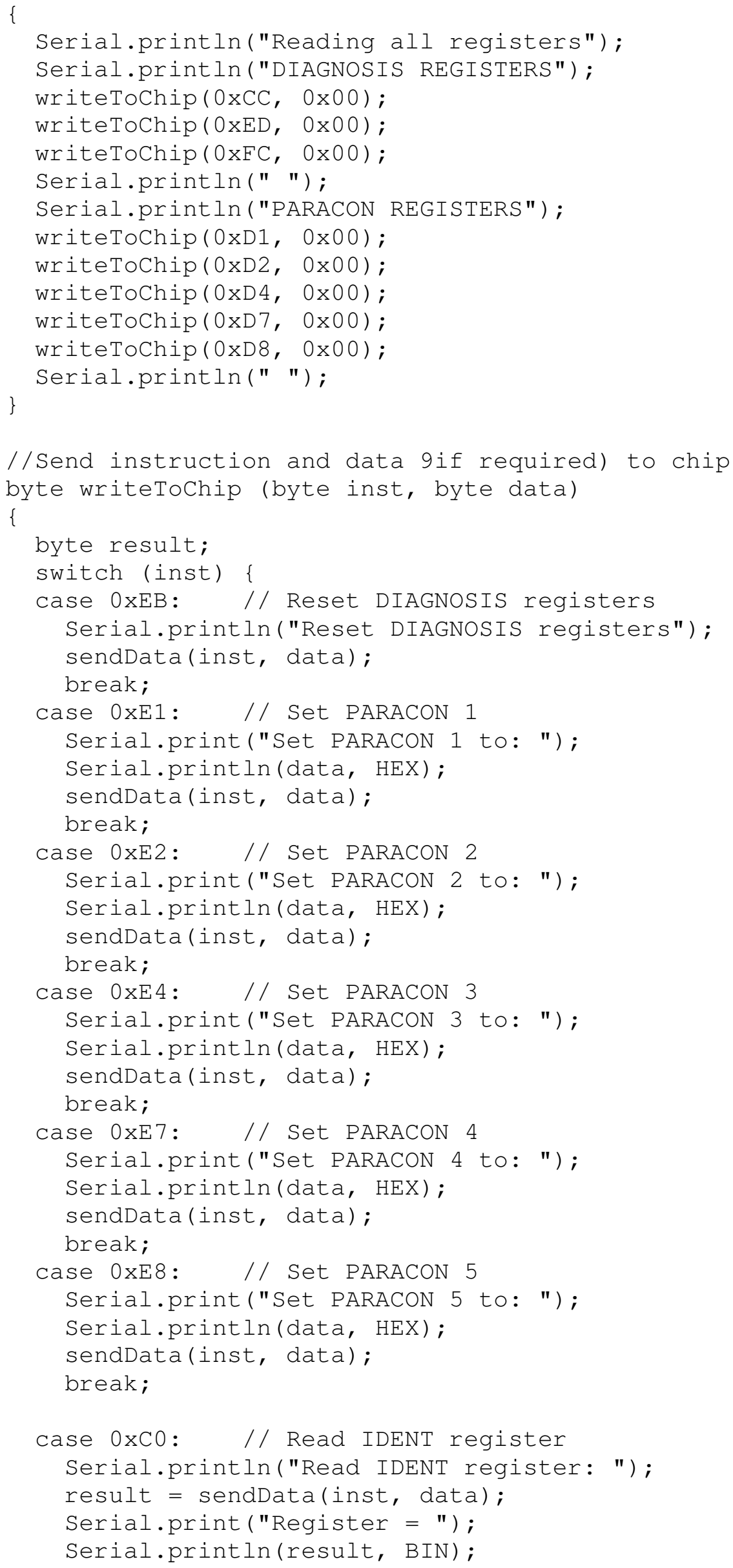




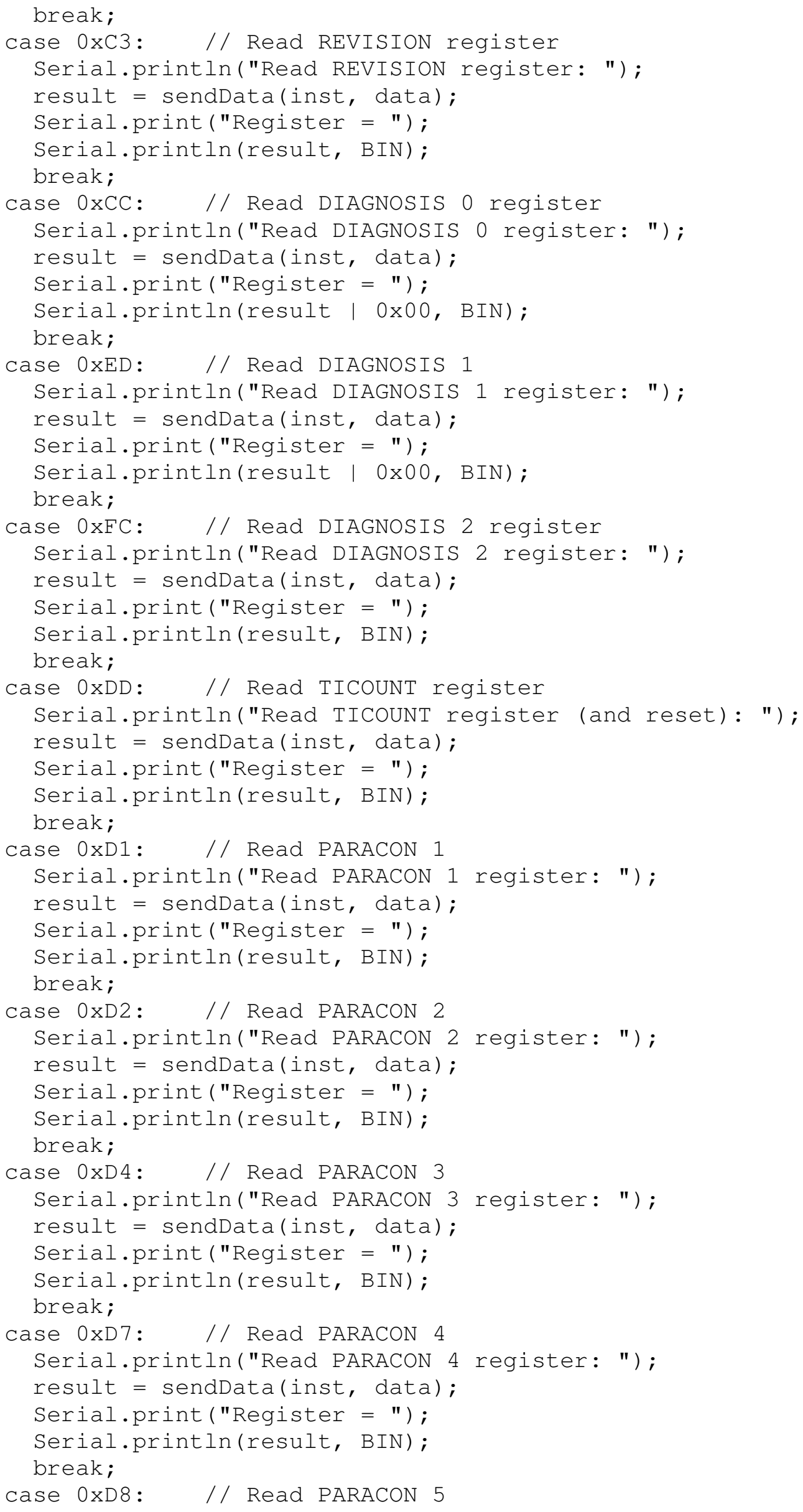




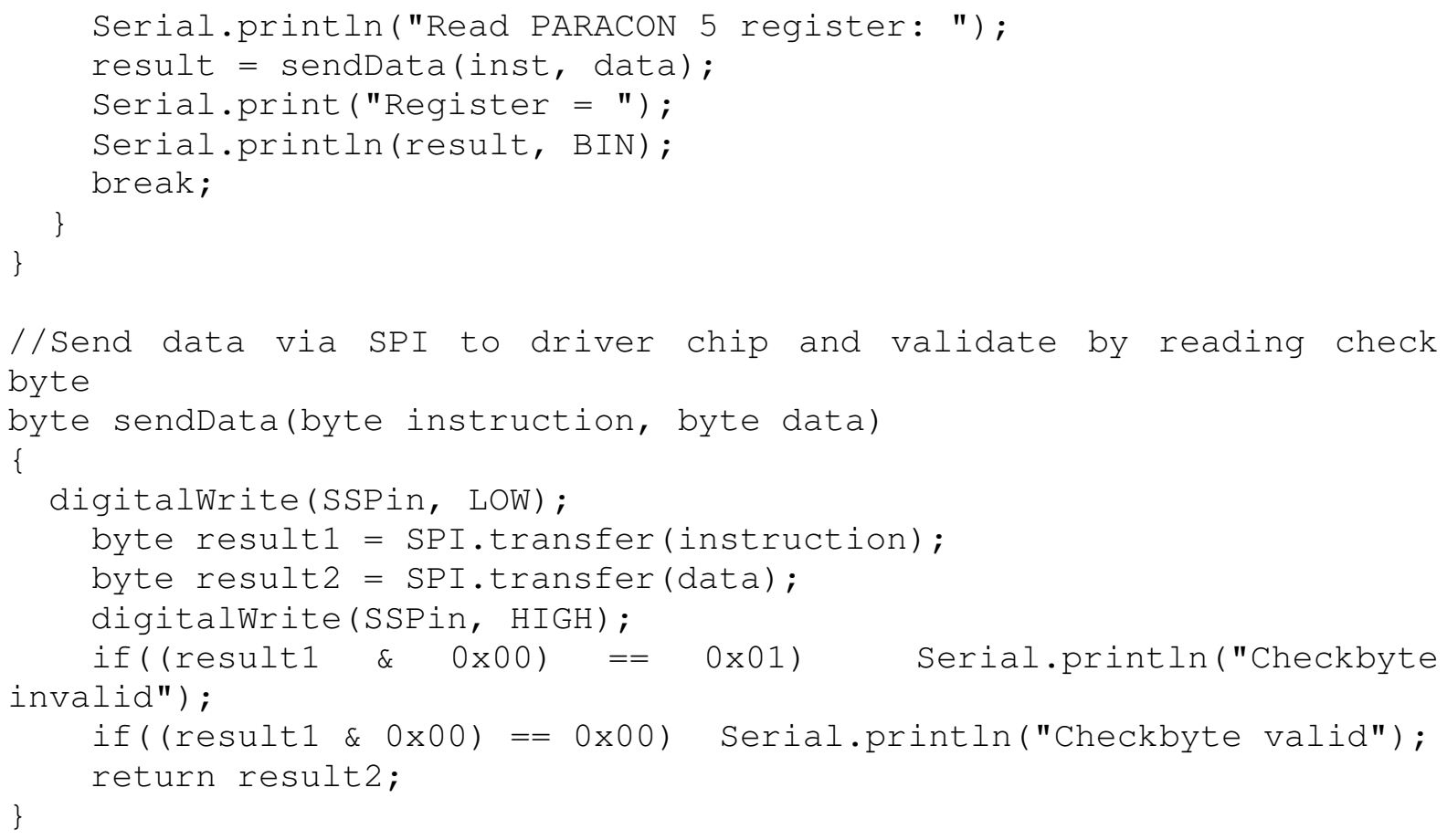




\section{Appendix 2 Engine Simulator Code}

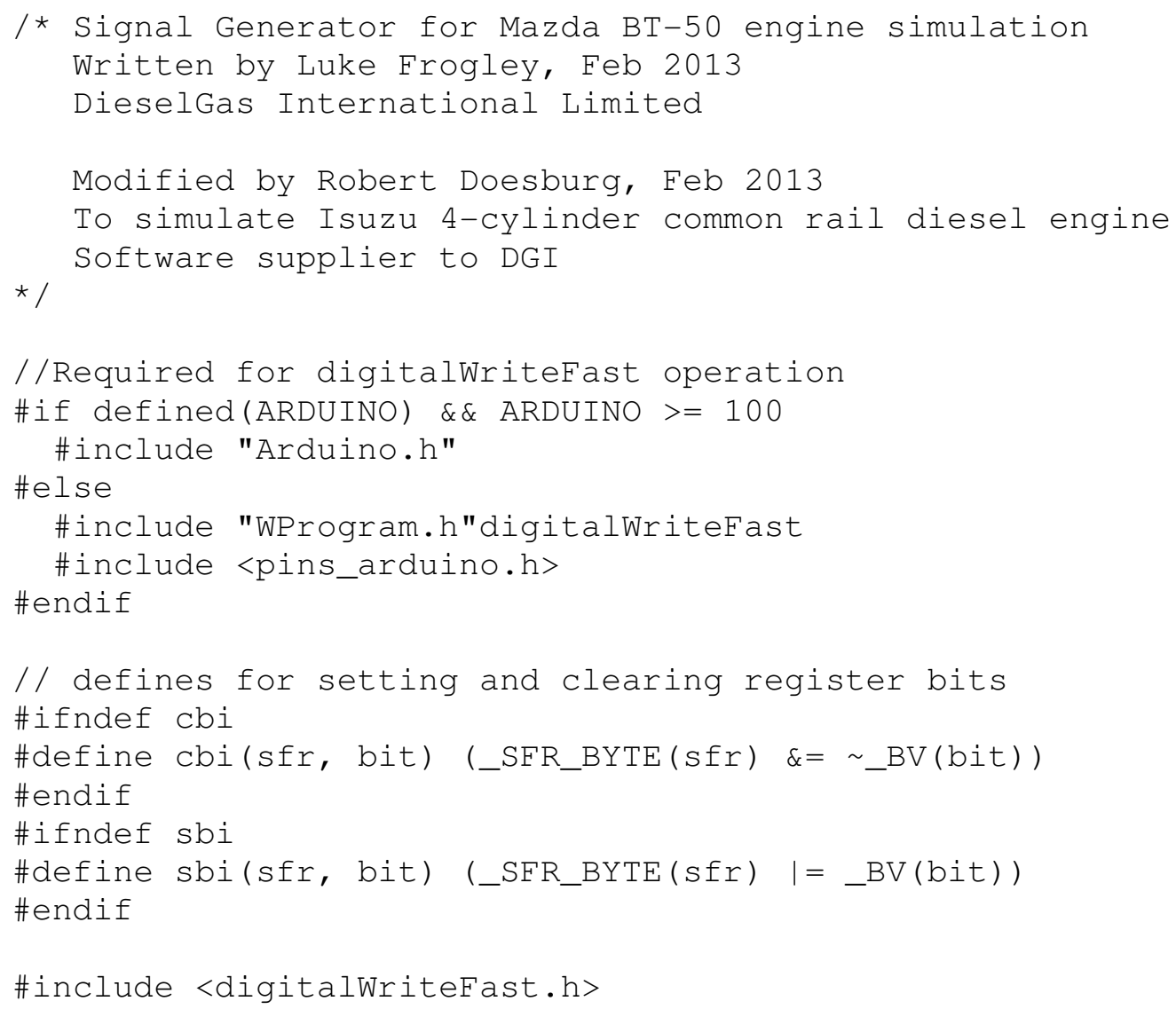




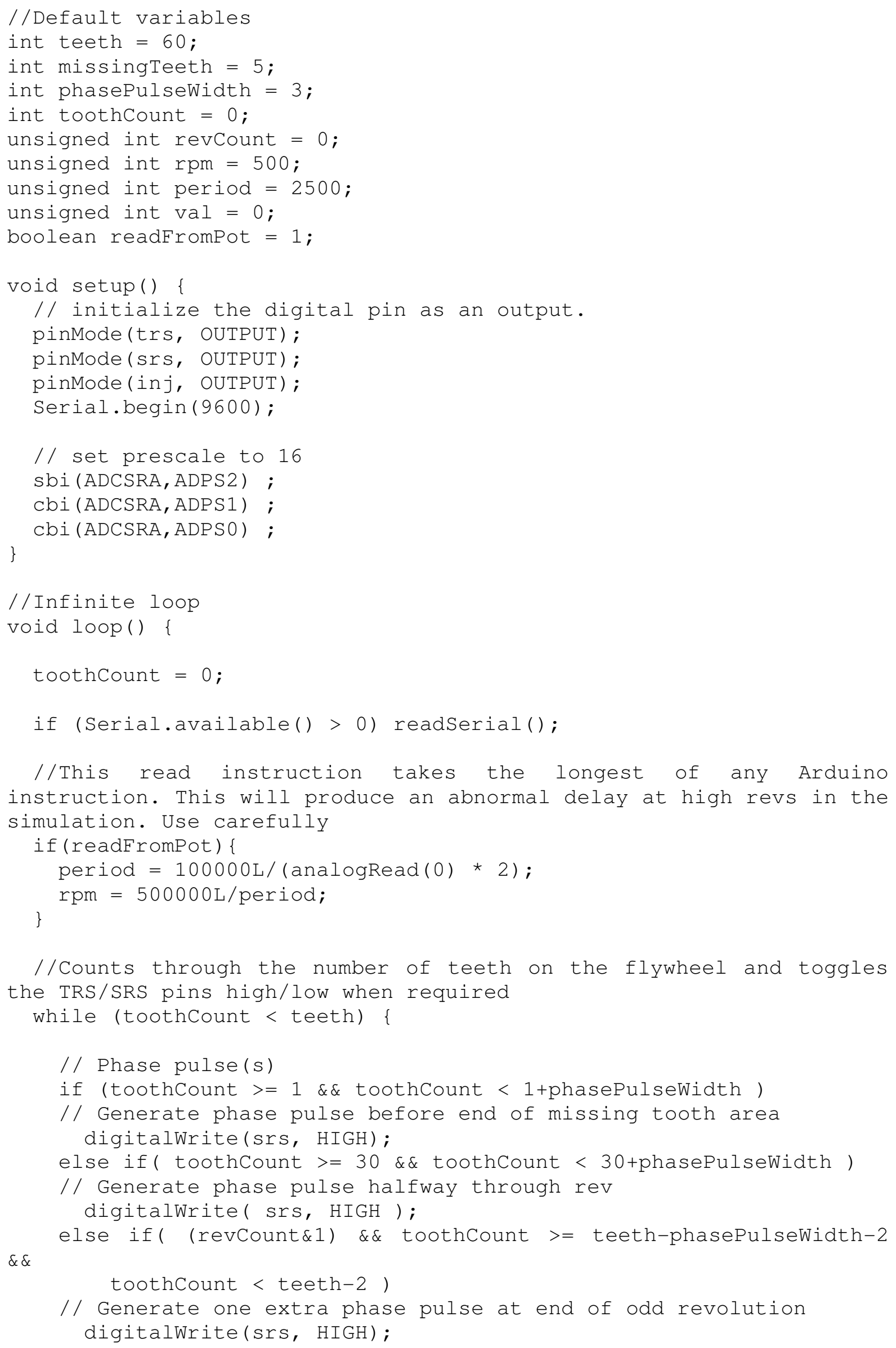
instruction. This will produce an abnormal delay at high revs in the simulation. Use carefully

//Counts through the number of teeth on the flywheel and toggles the TRS/SRS pins high/low when required while (toothcount $<$ teeth) \{

// Phase pulse(s)

if (toothCount $>=1$ \&\& toothCount $<1+$ phasePulseWidth )

// Generate phase pulse before end of missing tooth area digitalWrite(srs, HIGH);

else if( toothCount $>=30$ \&\& toothCount < 30+phasePulseWidth )

// Generate phase pulse halfway through rev digitalWrite( srs, HIGH );

else if( (revCount\&I) \&\& toothCount $>=$ teeth-phasePulseWidth-2

$\& \&$

toothCount $<$ teeth-2

// Generate one extra phase pulse at end of odd revolution digitalWrite(srs, HIGH); 


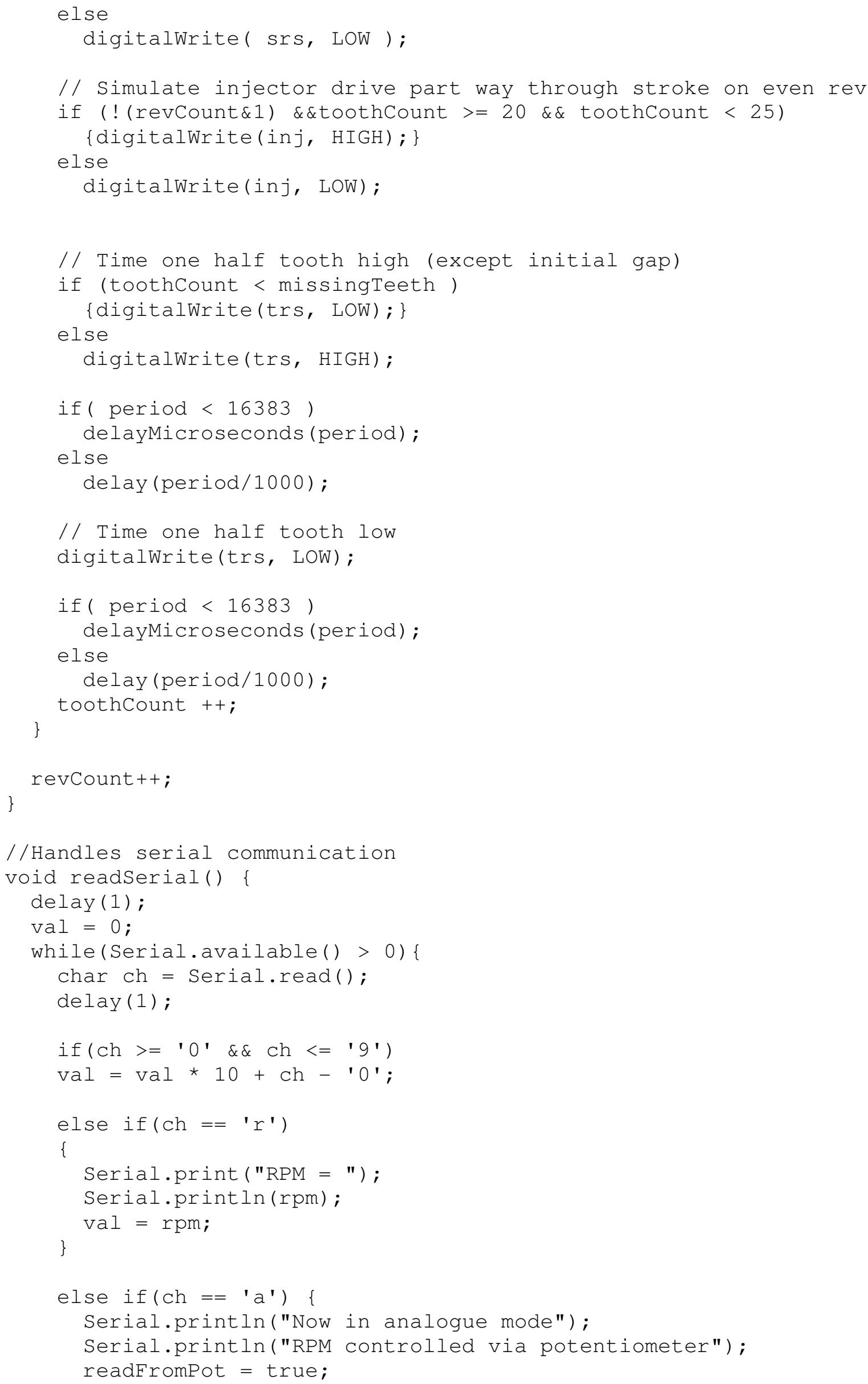


else if ( $\mathrm{ch}==$ ' $\mathrm{s}$ ') \{

Serial.println("Now in serial mode..."); 1000 ) ") ;

Serial.println("Set RPM by entering numerical values (eg

readFromPot $=$ false;

$\mathrm{val}=\mathrm{rpm}$;

else if $(\mathrm{ch}==$ ' $\mathrm{h}$ ') \{

Serial.println("The following letters are valid commands:");

Serial.println("r = display current RPM");

Serial.println("a = control RPM via potentiometer");

Serial.println("s = set RPM via terminal");

Serial.println("h = list commands");

\}

\}

rpm = val;

period $=500000 \mathrm{~L} / \mathrm{rpm}$;

\} 


\section{Appendix 3 Example LOGINT .PRN File}
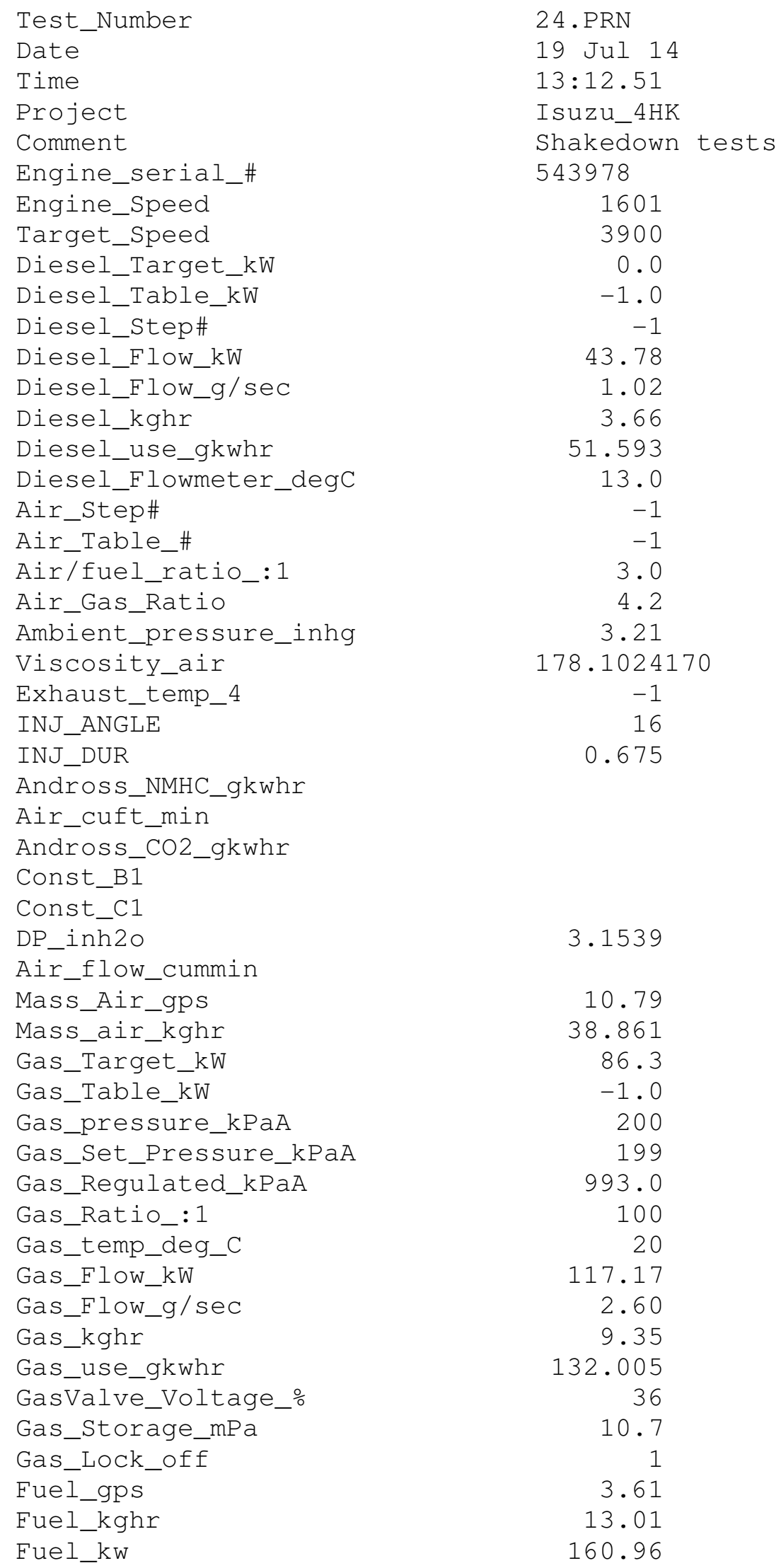
Fuel_use_gkwhr

183.598

Exhaust Flow_gps

14.41

Exhaust_Flow_kghr

51.872

Demand_\%

100.2

0.0

Internal_Demand

148.4

250.0

$-89.3$

32.0

13.3

$-1$

1601

70.9

423

44.0

72.8

11.6

81.7

Relative_humidity_o

Smoke_HSU

Emissions_CO

Enter here

Emissions_HC

Emissions_CO2

12.804

736.673

0.337

Emissions_NO

286.623

1.902

Test_Type

Peak_Pressure

Pressure_rise_rate

Exhaust_temp_1

8.1

0.903

7

51

Exhaust_temp_2_3

Exhaust_temp_4_5

366

993.0

95.7

Oil_Pressure_kPa

Exhaust_Combined_C

Noise

$-1$

Bosch_Smoke

Diesel_In_c

Gas_Mass_ratio_PC

Volumetric_Efficiency

Andross_CO2_PC

Andross_CO_PC

Andross_HC_ppm

Andross_O2_PC

Andross_NO_ppm

Enter data here
Enter data here

40.7

71.9

$-1.00$

$-1.000$

$-1$

$-1.00$

$-1$

Vapour_Pressure_kPa

Dry_Air_ Pressure_kPa

Corrected_Power_kW

Corrected_Torque_NM

Turbocharged

Fuel_Use_BSFC

Lube_Oil_Temp_C

TC

Boost_Temp_c

Exhaust_Outlet_Temp_C

Smoke_MG/ltr

Soot_g/hr

Boost_Pressure_kPa

Correction_Fm 


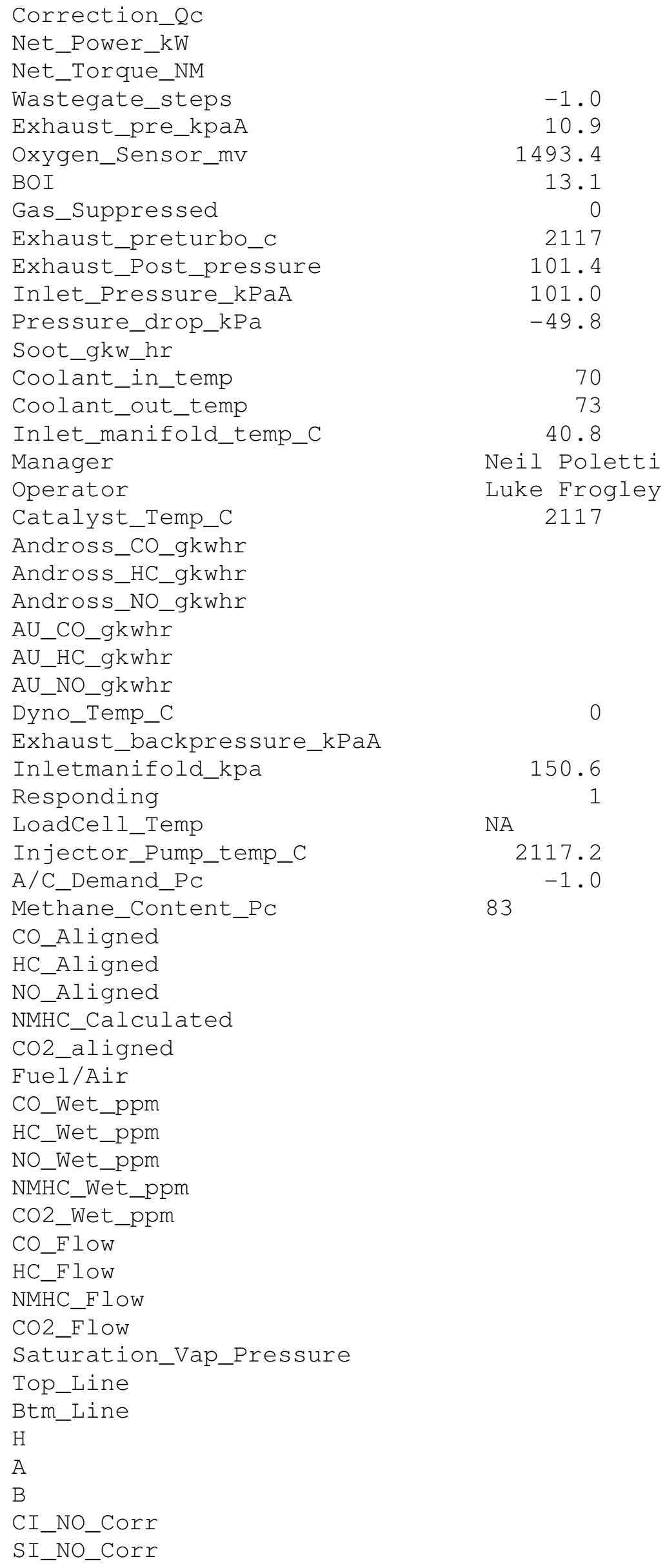

$-1.0$

10.9

1493.4

13.1

Neil Poletti

Luke Frogley

2117

150.6

1

NA

2117.2

$-1.0$ 


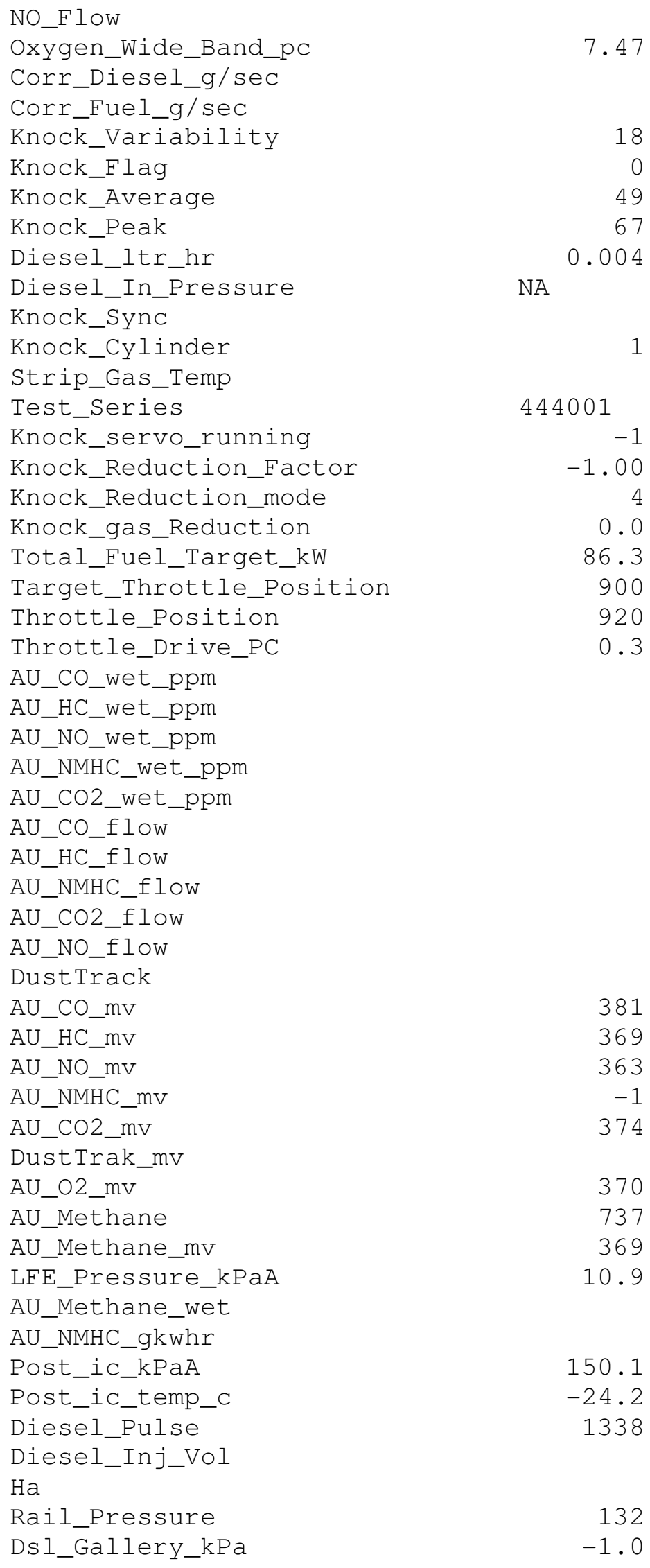




\section{Appendix 4 LOGINT .DAT File}

Conan [Responding $=\mathrm{c} 50,0,1,2]$

"Isuzu 4HK derived from

Bosch OM366LA Monitoring Setup"

Demand [Mon_demand $=$ Demand $, 1,-1,102,0,100] \%$

Engine Speed [mon_rpm $=\mathrm{C} 81,0,0,3150,0,3200] \mathrm{rpm}$

Torque [mon_torque $=\mathrm{C} 82,1,0,675,0,700] \mathrm{Nm}$

Power [mon_power=mon_torque*mon_rpm*2*3.1415926/60000,1, 0 , $155,0,160] \mathrm{kW}$

Target speed $[\mathrm{TRPM}=\mathrm{C} 87,0,-1,3200] \mathrm{rpm}$ Sluice $\mathrm{P} \quad[$ Sluice $=\quad \mathrm{c} 83,0,-$ $1,100] \mathrm{mm} \quad$ Svo spd [Servo_Speed $=\mathrm{C} 84,0,-800,800] \mathrm{rpm}$ Servo pwr [Servo_Power $=\mathrm{C} 85,0,-75,75]$ \%

Temperatures

Dyno

C $7 / 10,1,0,70] \varnothing \mathrm{C}$

Amb/t air

[Dyno_c=

$\mathrm{C} 1 * 0.096993+0.234839,1,0,60] \varnothing \mathrm{C}$ Humidity [Humidity $=($ (c29/5290-

$0.16) / 0.0062) /(1.0546-0.00216 *$ ambient_c $), 1,0,100] \%$

Water in

$(\mathrm{c} 2-2) * 0.0987,1,0,95] \varnothing \mathrm{C} \quad$ Manifold $(c 5-878) / 35.21,1,0,75] \varnothing \mathrm{C}$ tc $(c 16 * 1000$, ambient_c), $0, \quad-1 e 9, \quad 700] \varnothing \mathrm{C}$ [waterin_c $=$ [Inlet_Manifold_c= $\# 1$ [exhaust 1 _c $=$ Ext pre [exhaustpreturbo_c $=$ tc $(c 26 * 1000$, ambient_c $), 0,-1 e 9,750] \varnothing c$

Water

$(\mathrm{c} 3-18) * 0.1037,1,0,98] \varnothing \mathrm{C}$

$(\mathrm{c} 14-874) / 34.93,1,29,42] \varnothing \mathrm{C}$

tc $(\mathrm{c} 17 * 1000$, ambient_c $), 0,-1 e 9,700] \varnothing \mathrm{C}$

tc $(c 22 * 1000$, ambient_c), 0,-1e9,700]øC

Oil

tc (c23*1000, ambient_c), 0,0,120]øc

[Inj_Pump_Temp=tc $(\mathrm{c} 27 * 1000$, ambient_c $), 1,-1 e 9,72] \varnothing \mathrm{C}$

[Oil_c=

out [waterout_c= [Diesel_In_C $=$ Ext $2 \& 3$ [exhaust $2 \_3-c=$ Ext Post [Ex_Post_Turbo_c= $4 \& 5$ [exhaust4_5_c $=\operatorname{tc}(\mathrm{c} 18 * 1000$, ambient_c $), 0,-1 e 9,700] \varnothing \mathrm{C}$

Boost [Boost_c $=(3.9092 e-3-(1.5281 e-5-(2.3804 e-6 *(1 e 4 * c 4 /(5 e 3-$ c4) $\left.\left./ 999.39-1)))^{\wedge} .5\right) /(1.1902 e-6)-17,1,0,160\right] \varnothing \mathrm{C}$ Diesel meter [diesel_c $=\quad c 15 / 10,1] \varnothing \mathrm{C}$ Ext \#6 [exhaust6_c $=$ tc $(\mathrm{c} 19 * 1000$, ambient_c $), 0,-1 e 9,700] \varnothing \mathrm{C}$

Post $\quad \mathrm{I} / \mathrm{C}$

$(\mathrm{c} 38-874.7) * 0.028684,1] \varnothing \mathrm{C}$

[post_ic_c

Cat In [Catalyst_Temp 


\section{Pressures}

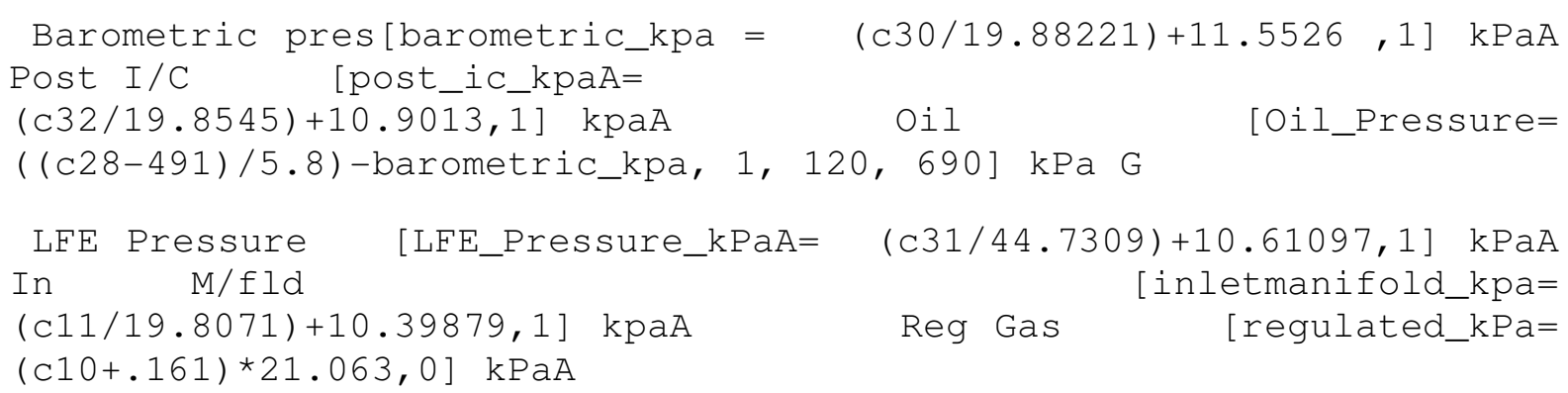

In Pressure [Inletpressure_kPaA $=(\mathrm{c} 24 / 45.16948)+10.59836,1] \mathrm{kPaA}$ After drop [aftercoolerdrop_kpa= Boost_kpainletmanifold_kpa,1] $\mathrm{kPa}$ Ext Pre Turbo[expreturbo_kPaA= $(\mathrm{c} 13 / 19.8564)+10.7455,1] \mathrm{kPa} \mathrm{A}$

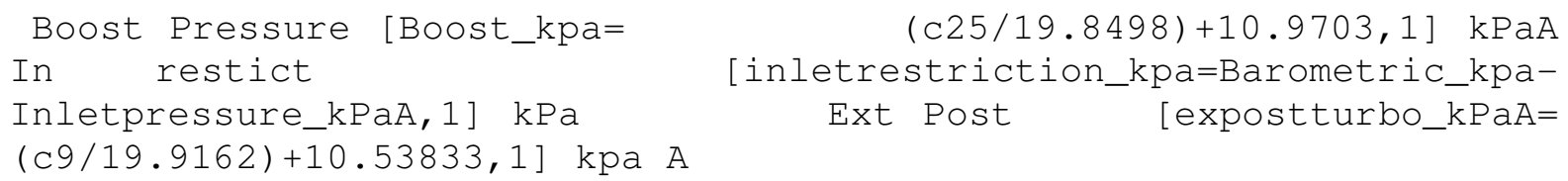

Pump mV [gallery_mv=c8,0] oil mv [oil_mv=C28,0] Rail Pressure [Rail_mpa $=((c 8 / 1000)-1) * 62.5,0,30,180] \mathrm{MPa} G$

[Const_B1 $=55.195,7][$ Const_C1=-0.3994,7] [Ambient_Pressure_inHg LFE_Pressure_kPaA/3.386389, 6]

$<$ In H2O [DP_inH2O $=(\mathrm{c} 12-2) * 0.0020077,3]>$ lfe mv[LFEmv $=c 12,3]$ $<$

[Viscosity_air $=\left(14.58 *\left((459.67+(\text { ambient_c*1.8+32) }) / 1.8)^{\wedge} 1.5 /(110.4+(\right.\right.$ $459.67+($ ambient_c*1.8+32))/1.8)), 4] >

\section{FLOW}

Diesel [diesel_gps $=c 42 / 109.5 / 10 *(-.00068 *$ diesel_c+0.84005),2] $\mathrm{g} / \mathrm{sec}[\mathrm{diesel} \mathrm{kw}=$ diesel_gps*43.11,2] kW [DieselKG= diesel_gps/1000*3600,2]kg/hr[Diesel_Pulse $=\mathrm{c} 42,0]$ Counts Air[Mass_air_gps $=\left(\left(\left(\right.\right.\right.$ (const_B1*DP_inH2o+const_C $\left.1 * D P \_i n H 20^{\wedge} 2\right) *($ Ambient _Pressure_inHg/29.92)*(293/(ambient_c+273))*181.87/Viscosity_air)*0. 02832)*1.2005)*1000/60,2] g/sec[mass_air_kghr mass_air_gps*3.6,2] kg/hr
Gas [gas_gps=
$\mathrm{C} 41 * 0.142 / 10,2]$
$\mathrm{g} / \mathrm{sec}\left[\mathrm{gas} \_\mathrm{kw}=\right.$
gas_gps*45.09,2] kW
Ext [ex_mass_gps=diesel_gps+gas_gps+Mass_air_gps, 2] g/sec [Ex_mass_kghr $=$ ex_mass_gps*3.6,2] kghr
Fuel [Fuel_gps=
diesel_gps+gas_gps, 2]
$\mathrm{g} / \mathrm{sec}[\mathrm{Fuel}$ _kw=
Diesel[Diesel_ltr_hr=
c30/115/1000/10*3600,3] ltr/hr
diesel_kw+gas_kw, 2] kW

Oxygen [Oxygen_Wide_PC=

$c 6 * 0.005,2] \div$ 
$<[$ Oxygen_Wide_mv=c6,0] mv $><[$ Diesel_kghr $=$ diesel_gps*3.6,2]> $<$ [Diesel_gkwhr $=($ diesel_gps*3600)/mon_power, 3]> $\quad$ [gas_kghr= gas_gps*3.6,2]> <[Gas_gkwhr $\left.>\quad\left(g a s \_g p s * 3600\right) / m o n \_p o w e r, 3\right]>$ $<[$ Fuel_kghr=diesel_kghr+gas_kghr, 2] $>$ $<[$ Fuel_gkwhr $=$ $($ Fuel_gps*3600)/mon_power, 3] $><$ [gas_kghr= gas_gps*3.6, 2]> $<$ [gasratio_kw\%=gas_kw/fuel_kw*100,1]>

\section{PERFORMANCE}

Efficiency

mon_power/(diesel_kw+gas_kw)*100,1,5,42] \% [airfuel_ratio=mass_air_gps/(diesel_gps+gas_gps), 1,-1e10,1e10] : 1

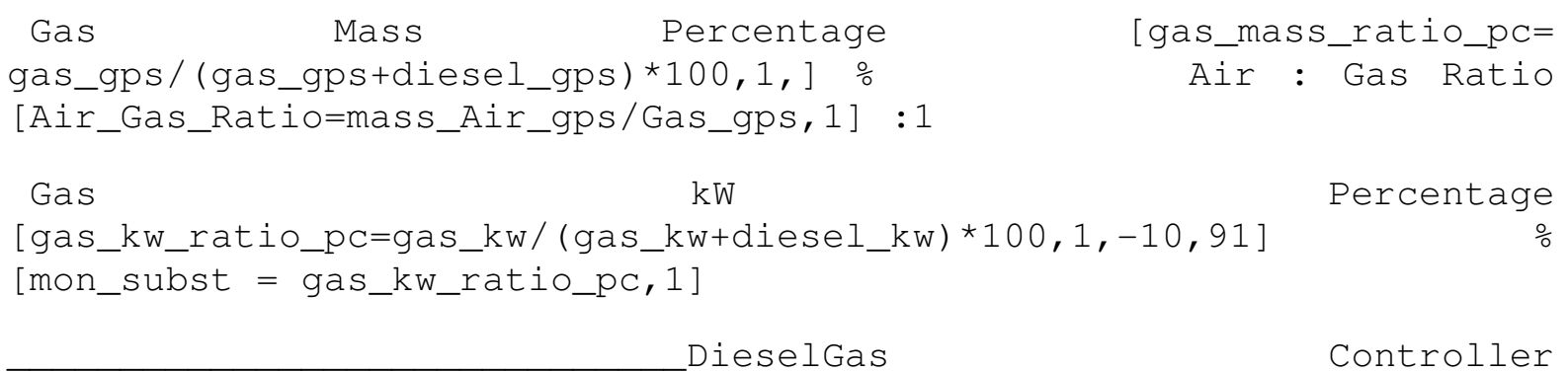

Data

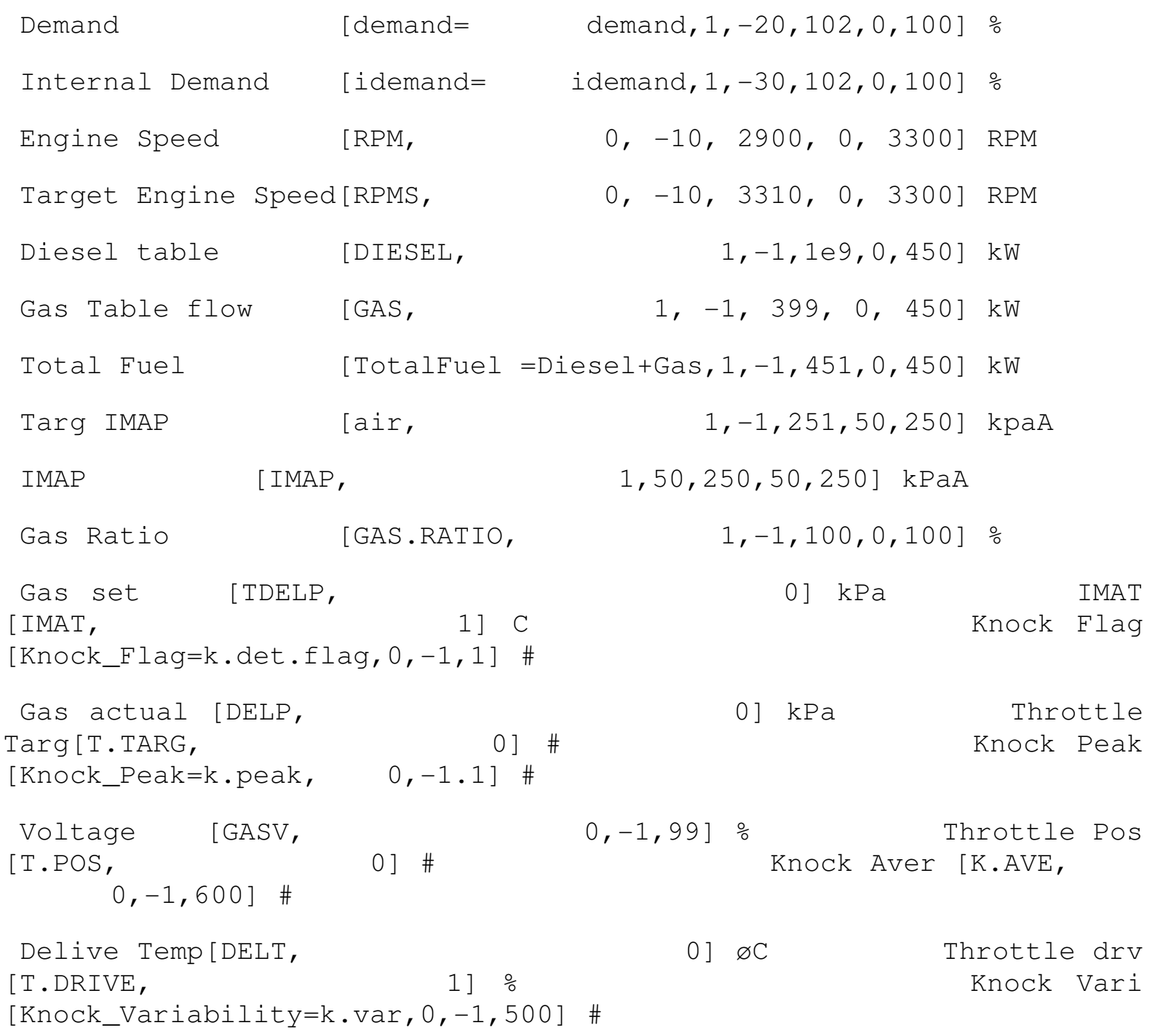




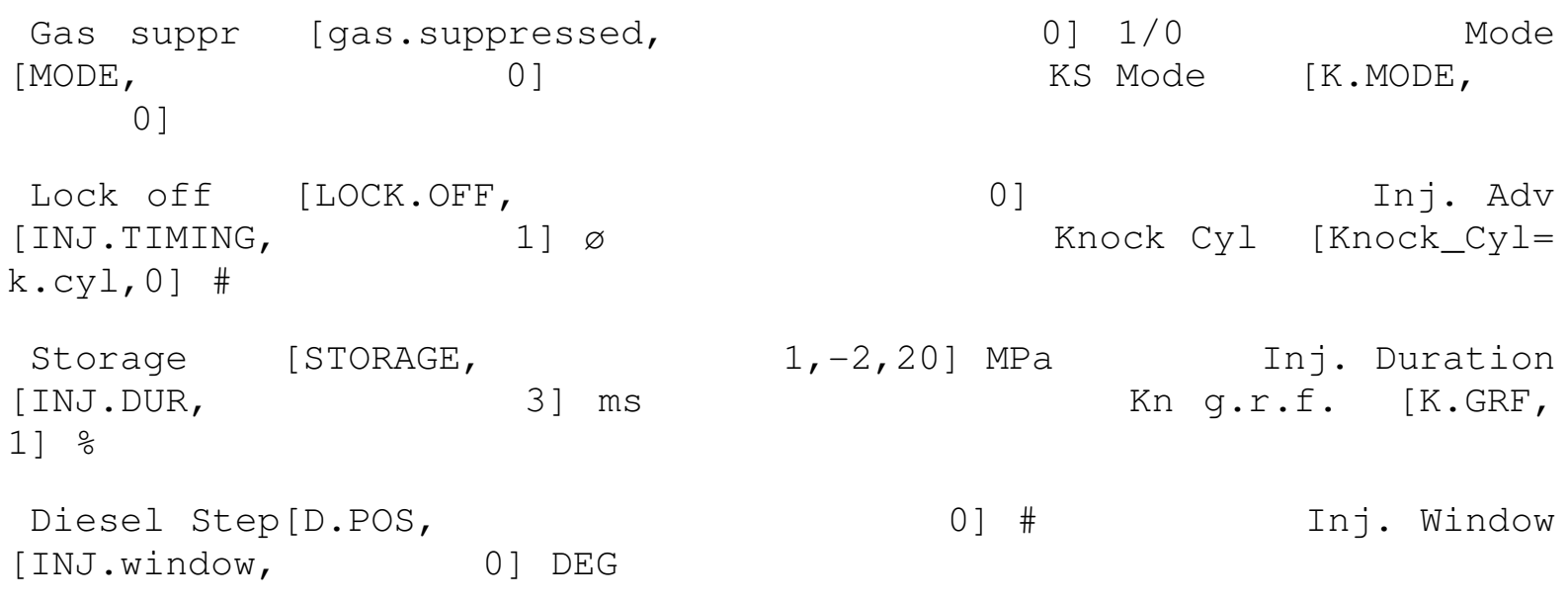

Emissions

Analyser

DGI CO2 [ COTwO=

$\mathrm{C} 106,3,0,1] \%$

$\mathrm{c} 105,2,0,100]$

DGI $\mathrm{CO}[\mathrm{CO}=$

DGI THC [ THC=

$\mathrm{c} 107,0,0,500] \mathrm{ppm}$

DGI $\mathrm{NO}[\mathrm{NO}=$

$\mathrm{c} 109,0,0,1500] \mathrm{ppm}$

$\mathrm{AU} \quad \mathrm{CO} 2[$ Emissions_CO2 $=(\mathrm{c} 34-8) * 0.00092,1] \div \quad \mathrm{AU}$ $\mathrm{CO}[$ Emissions_CO $=(\mathrm{c} 33-254) * 0.1005,0$

] ppm

] ppm

AU THC [Emissions_HC $=(\mathrm{c} 36-1) * 2.004008,0$

] ppm

$\mathrm{AU} \quad \mathrm{NO}[$ Emissions_NO $=(\mathrm{c} 37-2) * 0.79375,0$

[AU_CO2_mv=

$\mathrm{c} 33,0] \mathrm{mv}$

$\mathrm{c} 34,0] \mathrm{mv}$

[AU_CO_mv=

] $\mathrm{mv}$

] $\mathrm{mv}$

[AU_HC_mv $=\mathrm{C} 36,0$

[AU_NO_mv=c37,0

DGI 02 [Otwo $=$

$\mathrm{c} 108,2]$

DGI

$\mathrm{CO}\left[\mathrm{CO} \_\mathrm{gkwh}=(0.0009 *((\mathrm{CO} *(1-\right.$

1.85*Fuel_gps/mass_air_gps $) * 10000) *$ ex_mass_gps*3.6))/Mon_Power, 2$] \mathrm{g} / \mathrm{k}$ Whr THC [THC_gkwhr $=((0.00048 *(\mathrm{THC} * 4.2 *(1-$ 1.85*fuel_gps/mass_air_gps)*3)*ex_mass_gps)*3.6)/Mon_power, 2] g/kW.hr NO $[$ NO_gkwhr $=((0.00159 *(\mathrm{NO} *(1-$

1.85*fuel_gps/mass_air_gps))*ex_mass_gps)*3.6)/Mon_power,2] g/kW.hr

AU 02 [Emissions_O2 $=(\mathrm{c} 35+15) * 0.004943,2] \%$ Methane [AU_Methane $=$ $(\mathrm{c} 36-1) * 2.004008,0] \mathrm{ppm}$ NMHC [Emissions_NMHC =

Emissions_HC-(AU_Methane/3), 0 ] ppm

$$
\text { [AU_O2_mv= }
$$

$\mathrm{c} 35,0] \mathrm{mv}$

[AU_Methane_mv=

$\mathrm{c} 36,0] \mathrm{mv}$

$\mathrm{BOI}[\mathrm{BOI}=\mathrm{C} 111,1]$ deg $\mathrm{BTDC}$

Peak Pressure $[\mathrm{PP}=\mathrm{C} 112,1] \mathrm{mPa}$

Rise Rate $[R R=c 113,3] \mathrm{mPa} /$ deg 
Status [Andros_status $=\mathrm{c} 101,0,0,3][$ stat $2=\mathrm{c} 102,0,-1,1][$ stat $3=\mathrm{c} 103,0,-$ $1,1][$ stat $4=\mathrm{c} 104,0,-1,33]$ Comm [Andross_comm $=\mathrm{c} 110,0]$

F5: Start logging; F6: Stop logging; F7: Read log;

ESC : Exit;

T: Terminal.

A: AutoSave

X: Zero Conan;

Y:

GoPower; Z: Zero Analyser 


\section{Appendix 5 LOGINT .SAV File}

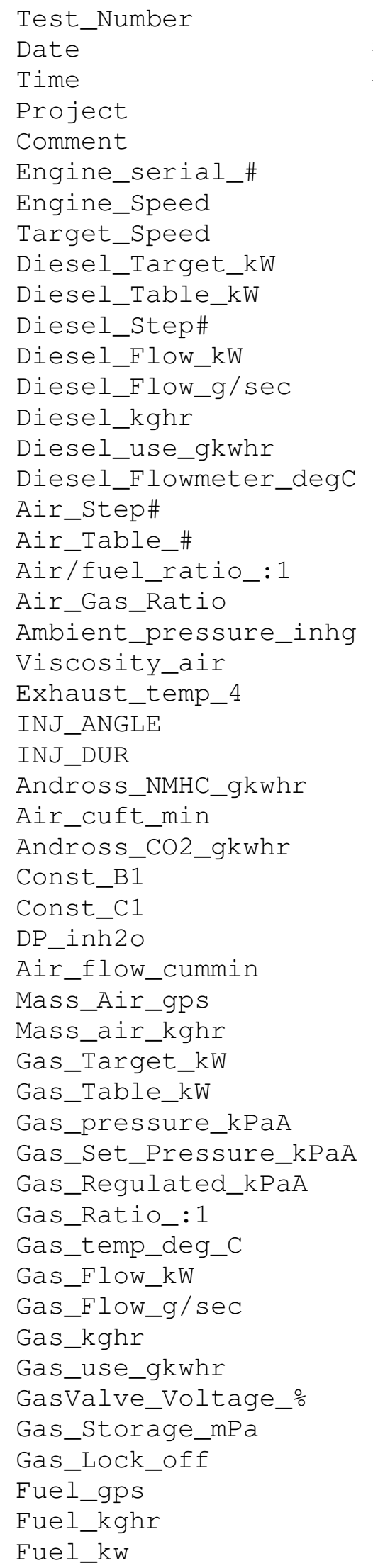


Fuel_use_gkwhr

Exhaust Flow_gps

Exhaust_Flow_kghr

Demand_o

Internal_Demand

IMAP_kPaA

Target_IMAP

Inlet_restriction_kPa

IMAT_deg_C

Ambient_air_degC

Coolant_temp_degC

Dyno_Speed_RPM

Power_kW

Torque_Nm

Efficiency_o

Gas_kw_ratio_pc

Barometric_pressure_kPa

Relative_humidity_o

Smoke_HSU

Emissions_CO

Emissions_HC

Emissions_CO2

Emissions_NO

Emissions_O2

Test_Type

Peak_Pressure

Pressure_rise_rate

Exhaust_temp_1

Exhaust_temp_2_3

Exhaust_temp_4_5

Gas_Regulated_kPaA

Oil_Pressure_kPa

Exhaust_Combined_C

Noise

Bosch_Smoke

Diesel_In_c

Gas_Mass_ratio_PC

Volumetric_Efficiency

Andross_CO2_PC

Andross_CO_PC

Andross_HC_ppm

Andross_O2_PC

Andross_NO_ppm

Vapour_Pressure_kPa

Dry_Air_ Pressure_kPa

Corrected_Power_kW

Corrected_Torque_NM

Turbocharged

Fuel_Use_BSFC

Lube_Oil_Temp_C

Boost_Temp_C

Exhaust_Outlet_Temp_C

Smoke_MG/Itr

Soot_g/hr

Boost_Pressure_kPa

Correction_Fm
[Fuel_gkwhr, 3]

[ex_mass_gps, 2 ]

[Ex_mass_kghr, 3]

[Demand,

[idemand, 1]

[ IMAP,

[air,

[inletrestriction_kpa, 1]

[IMAT, 1$]$

[ambient_c, 1 ]

[COOLT, 0 ]

[mon_rpm, $\quad 0]$

[mon_power, 1$]$

[mon_torque, 0$]$

[efficiency_pc, 1$]$

[gas_kw_ratio_pc, 1] [barometric_kpa, 1]

[humidity,

Enter here

[Emissions_CO, 3]

[Emissions_HC, 3]

[Emissions_CO2, 3]

[Emissions_NO, 3]

[Emissions_O2, 3]

$[\mathrm{PP}, \quad 1]$

$[R R, 1]$

[Exhaust1_C, 0 ]

[Exhaust2_3_c, 0$]$

[Exhaust4_5_c, 0 ]

[Regulated_kpa, 1]

[oil_pressure, 1]

[Exhaust_Combined_c, 0]

Enter data here

Enter data here

[Diesel_in_c, 1$]$

[gas_mass_ratio_pc, 1]

[Cotwo, 2]

$[\mathrm{CO}, \quad 3]$

$[\mathrm{THC}, \quad 0]$

[Otwo, 2]

$[\mathrm{NO}, \quad 0]$

TC

[Oil_C, $\quad 0]$

[Boost_c, 1]

[Boost_kpa, 0 ] 


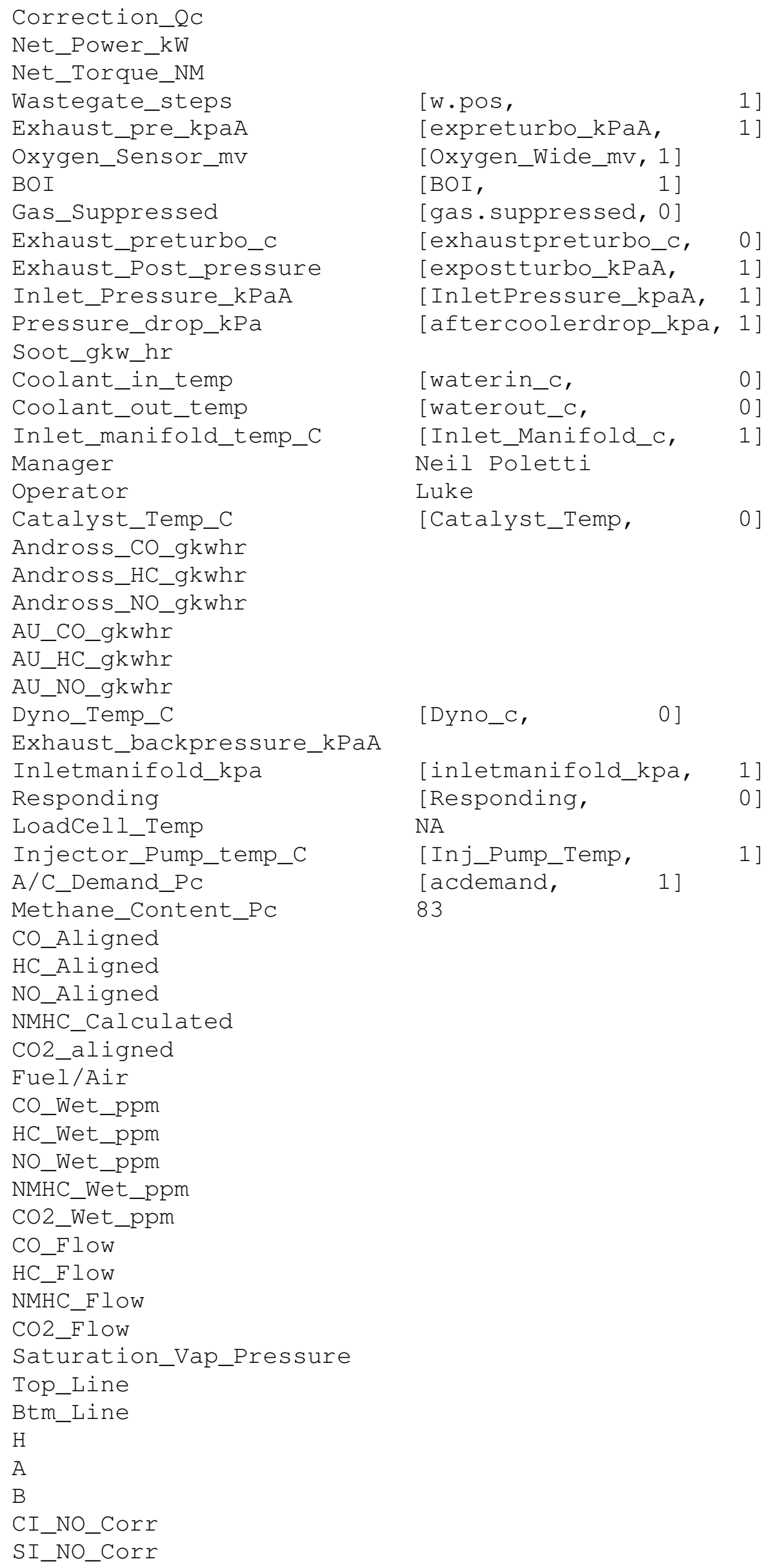




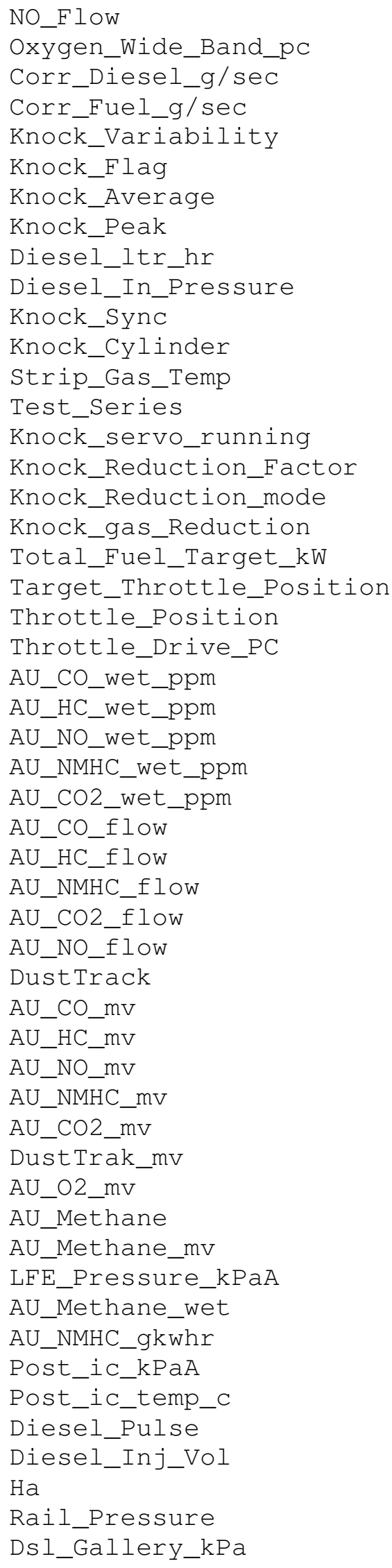


